\title{
AERODYNAMIC ANALYSES OF SKINS FOR MORPHING WINGS
}

by

\author{
Fadi Magdy R Mishriky \\ Bachelor of Science, German University in Cairo (2012) \\ Master of Science, Dresden University (2014)
}

\author{
A dissertation \\ presented to Ryerson University \\ in partial fulfillment of the \\ requirements for the degree of \\ Doctor of Philosophy \\ in the program of \\ Aerospace Engineering \\ Toronto, Ontario, Canada, 2018 \\ (C) Fadi Magdy R Mishriky, 2018
}




\section{AUTHOR'S DECLARATION FOR ELECTRONIC SUBMISSION OF A DISSERTATION}

I hereby declare that I am the sole author of this dissertation. This is a true copy of the dissertation, including any required final revisions, as accepted by my examiners.

I authorize Ryerson University to lend this dissertation to other institutions or individuals for the purpose of scholarly research.

I further authorize Ryerson University to reproduce this dissertation by photocopying or by other means, in total or in part, at the request of other institutions or individuals for the purpose of scholarly research.

I understand that my dissertation may be made electronically available to the public. 


\title{
AERODYNAMIC ANALYSES OF SKINS FOR MORPHING WINGS
}

\author{
Fadi Magdy R Mishriky \\ Doctor of Philosophy, Aerospace Engineering, Ryerson University, Toronto (2018)
}

\begin{abstract}
Despite years of research and development in aviation, nearly all aircraft's wings are designed according to the same model of a rigid structure equipped with few discrete control surfaces. This design performs well at a single flight regime and suffers degradation in its aerodynamic properties at other regimes. For that reason, and with the advancements that have been witnessed in the field of smart material and adaptive structures, a biomimetic concept that challenged this rigid model has been revived. This concept is morphing aircraft. Aircraft morphing technology enables a single air vehicle to undergo substantial geometric changes in-flight, with the purpose of increasing efficiency, versatility, and mission performance.

One of the most challenging tasks in this research topic is to design, implement and test a morphing skin that can compromise between flexibility to ensure low actuation requirements, and high stiffness to carry all the aerodynamic loads. While several studies have focused on the mechanical designs of morphing skins, studying the aerodynamic effects of these models have been largely ignored. Infinitesimal discontinuities resulting from the overlapping panels of sliding skins, and small ripples of stretchable skins can affect the stability of the viscous boundary layer which dictates the overall aerodynamic performance.

In this thesis, the aerodynamics of three types of morphing skins are studied. The first two fall under the category of sliding morphing skin where several rigid panels overlap and slide against each other during morphing. During morphing, the panels can either have backward-facing steps between them, gaps, or both. Despite their geometric similarity, the aerodynamics of backwardfacing steps are quite distinguished from panels with gaps between them.

An aerodynamically less invasive design for morphing skin is the stretchable skin. This design consists of two layers, an underlaying supporting structure, and an outer sealant layer. The underlaying layer is manufactured from a modified zero-Poisson ratio cellular structure with major ribs extending along the chord-wise direction, and minor ribs joining these major ribs. The outer layer consists of a highly anisotropic elastomer composite with Silicone rubber used as the matrix material and reinforced with carbon fibers along the chord-wise direction. During morphing, the flexible skin forms wrinkles along the main direction of morphing which significantly affect the aerodynamic properties of the morphing wing.
\end{abstract}


The aerodynamics of each one of these three designs is numerically studied using the high fidelity commercial computational fluid dynamics (CFD) code FLUENT 15.0. The first design with the backward-facing steps distributed along the chordwise direction of the airfoil showed to have a degrading effect on the aerodynamics of the wing. Regardless of the step size, the boundary layer experiences a transition from laminar to turbulent state at the step edge. At $\operatorname{Re}=5.7 \mathrm{e} 6, \mathrm{M}=0.2$, and $\alpha=2.5^{\circ}$, a drop of $21.1 \%$ in value of the lift coefficient and an increase of $120.8 \%$ in the drag coefficient were observed in case of a step located at $25 \%$ of the chord length on the upper surface of the airfoil. These effects can be mitigated by either shifting the step location towards the trailing edge or decreasing the step depth. For a step located on the lower surface of the airfoil, the lifting forces increased by at least $11 \%$ due to decreasing the airfoil thickness on the pressure side. The drag coefficient also increased by $63.46 \%$ for a step located at $25 \%$ of the chord length on the lower surface of the airfoil. This value decreases to $25.96 \%$ when the step is shifted to $75 \%$ of the chord length. A degraded near stall behavior was observed for a step located at either side of the airfoil. The separation of the flow at the step edge promotes early stall of the airfoil. These results show that distributing the sliding panels of the morphing skin in a sequential order is not an aerodynamically viable solution.

When the panels are separated with gaps, the aerodynamic performance was found to be different. It was observed that in some cases, the boundary layer maintained its laminar state while travelling over the cavity, and in other cases, the boundary layer experienced a transition over the cavity vicinity. To investigate the cavity parameters that influence the transition of the boundary layer, a parametric study is performed over a wide range of flow conditions and cavity dimensions. It is found that the boundary layer by-passes the cavity and maintains its laminar state when $L / \theta *$ $\sqrt{R e_{\theta}} \sim<600$, where $\mathrm{L}$ is the cavity length, $\theta$ is the momentum thickness of the boundary layer, and $R e_{\theta}$ is the Reynolds momentum thickness based $\theta$ on at the cavity leading edge. This formula is used to design an airfoil with sliding morphing panels that have the same performance as the clean airfoil, making this design a favorable design for morphing skins.

The third design is the flexible morphing skin. With an underlaying supporting structure and a highly anisotropic flexible outer layer, the flexible skin fulfilled all the kinematic and structural requirements of a successful morphing skin. However, during morphing, wrinkles are formed on the upper and lower surfaces of the morphing wing. Regardless of its location, shape or size, the introduced wrinkles alter the boundary layer state and behavior which significantly affect the overall aerodynamic performance of the morphing wing. The aerodynamic effects of introducing a single wrinkle to the upper surface of a morphing wing is numerically studied. At $\operatorname{Re}=5.7 \mathrm{e} 6, \mathrm{M}$ $=0.2$, and $\alpha=0^{\circ}$, results showed that introducing a single wrinkle to the upper surface of a NACA 2412 airfoil has dropped the lift coefficient by as much as $34.7 \%$ of the clean airfoil value, increased the drag coefficient by $267.9 \%$ and dropped the lift-to-drag ratio by $75.6 \%$ when the 
wrinkle is at the first quarter of the chord length. Shifting the wrinkle towards the trailing edge of the airfoil has mitigated these effects but did not eliminate them.

By comparing the results of the three designs, the sliding morphing skin with its panels arranged in a staggered manner has fulfilled the structural and kinematic requirements of a morphing skin while maintaining an aerodynamic performance similar to the clean airfoil if designed properly. 


\section{ACKNOWLEDGEMENTS}

First and foremost, I would like to express my deep and sincere gratitude to my amazing supervisor Dr. Paul Walsh for his continuous support, guidance and patience. He is a great mentor, inspiration, and a role model. His selfless time and care made me always want to achieve more and more. He has given me all the freedom to pursue my research, while providing me with his invaluable guidance that kept me on track of my research. It was a great privilege and honor to work and study under his guidance. I owe him my success and shall always be his grateful student.

I wish to sincerely thank Dr. Jeff Xi for his proficient leading of this morphing wing project. His patient guidance, encouragement and advice to our team was essential for the completion of this work. I cannot forget the valuable contribution that Aaron Yu, Dr. Daniel Finistauri and Dr. Amin Moosavian gave to the mechanical design of the morphing skin and morphing wing.

I would also like to thank Dr. Goetz Bramesfeld whose office was across from my working laboratory. His opened office door always delighted me, as did his enjoyable and cheerful conversation. I appreciate all his words of encouragement, and his professional opinion about parts of my research.

Next comes the dearest to my heart; thanking my parents and beloved ones. Words can't express how grateful I am to my mom and dad. Your countless years of ceaseless sacrifice, continuous support, unconditional love and nonstop prayers are what made me reach that far. I owe you everything in my life, I owe you being who I am today. Also, I express my thanks to my beautiful sister Phoebe for believing in me. Her keen interest in me completing this degree was a great motivation for me to keep going.

Last but not least, I would like to thank the Almighty God for giving me the ability, strength, knowledge and perseverance to complete this research. Without showering me with your limitless blessings, this work would not have come to existence. I feel Your guidance in every step I take, and every decision I make. My trust in you is the source of my strength. Thank you, Lord Jesus. 
TABLE OF CONTENTS

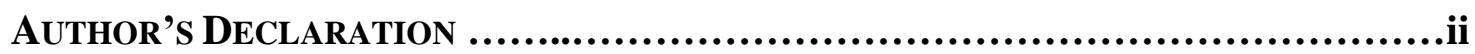

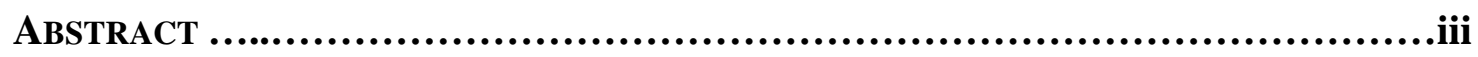

ACKNOWLEDGEMENTS ............................................................vi

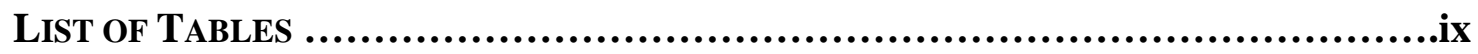

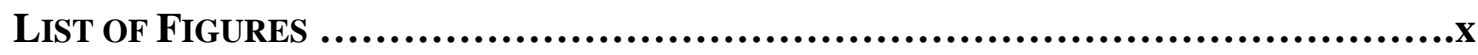

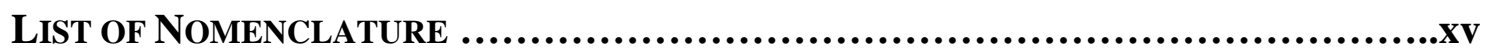

LIST OF ABBREVIATIONS AND ACRONYMS ..........................................xviii

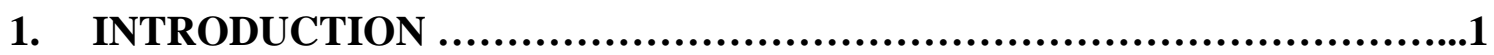

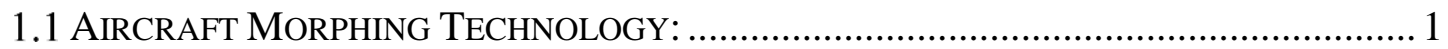

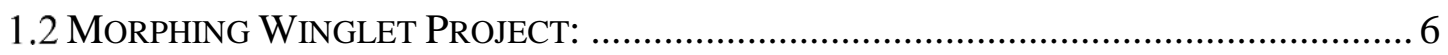

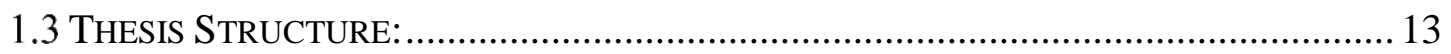

2. COMPUTATIONAL FLUID DYNAMICS AND NUMERICAL MODELING16

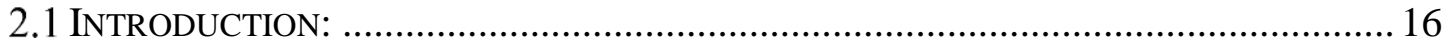

2.2 VERIFICATION OF THE GRADIENT RECONSTRUCTION METHOD............................... 21

2.3 VALIDATION OF THE NACA 2412 CFD RESULTS ................................................... 40

3. FLOW OVER TRENCHES OF SLIDING PANELS .........................................42

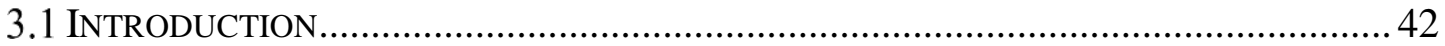

3.2 NUMERICAL Model VALIDATION …………………....................................... 45

3.3 VALIDATION Of THE Computational Model ………….................................... 51

3.4 NUMERICAL PREDICTION OF BOUNDARY LAYER TRANSITION …….......................... 58

3.5 PRACTICAL APPLICATIONS FOR MORPHING SKINS .................................................63

4. AERODYNAMICS OF OVERLAPPING SLIDING SKIN WITH BACKWARD-

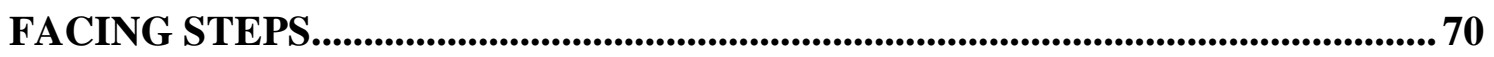

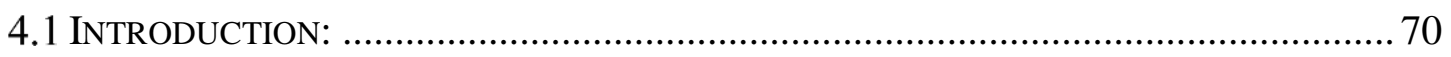

4.2 Aerodynamics of Backward-Facing Steps on the UpPer Surface of an Airfoll: .73

4.3 Aerodynamics of Backward-Facing Steps on the Lower Surface of AN Airfoll:

5. DESIGN AND AERODYNAMICS OF FLEXIBLE MORPHING SKIN..... 107

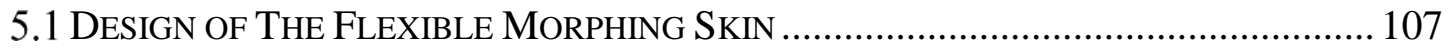

5.2 AEROdYNAMICS OF FLEXIBLE MorPHING SKIN................................................. 122

6. CONCLUSION, CONTRIBUTIONS AND FUTURE WORK .......................136

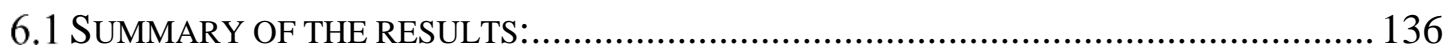


6.2 CONTRIBUTIONS:.

6.3 FUTURE WORK:.

APPENDICES

145

APPENDIX A: STEPS TO RUN A FLUENT CASE ON SHARCNET SERVER 145

APPENDIX B: GENERAL GUIDELINES IN GENERATING STRUCTURED GRIDS FOR AIRFOILS147

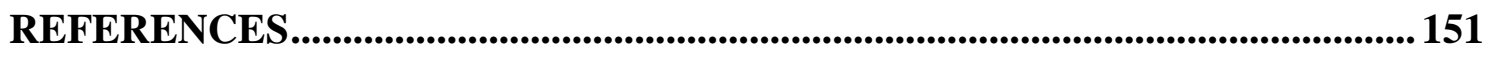




\section{LiST OF TABLES}

Table 2.1. Approximate wall clock time of different computational cases.............................. 19

Table 3.1. Sample of the matrix created to predict the factors that triggers the transition of the boundary layer over cavities.

Table 3.2. Locations of trenches installed over the NACA 2412 airfoil, the momentum thickness at these locations, the Reynolds momentum thickness, shape factor, and the maximum allowable

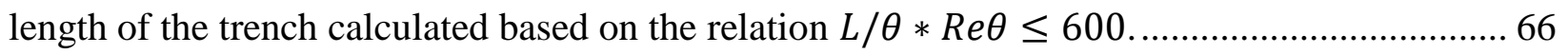

Table 4.1. Values of the $\mathrm{cl}, \mathrm{cd}$ and $\mathrm{L} / \mathrm{D}$ at different step angles $\beta$....................................... 89

Table 4.2. Values of $\mathrm{cl}$, $\mathrm{cd}$ and $\mathrm{L} / \mathrm{D}$ at different step angles. .............................................. 104

Table 5.1. Properties of the four composite samples estimated using the rule of mixture for

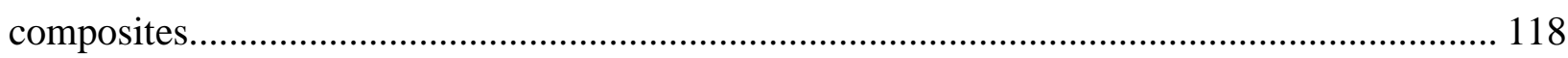

Table 5.2. Results of the out-of-plane deflection test. ..................................................... 120 


\section{LIST OF FiguRES}

Figure 1.1. Spider plot comparing the performance of a fixed-geometry Firebee, a morphing airfoil Firebee and a morphing planform Firebee. Reproduced from [4] ....................................................2

Figure 1.2. Classification of common morphing motions of wings as classified by Sofla et al. [12]. .5 Figure 1.3. A four degrees-of-freedom morphing wing using variable geometry truss mechanism to achieve (a) span, (b) sweep, (c) twist and (d) cant motions. Reprinted from [13]............................ 6

Figure 1.4. Proposed design for a 2 module VGTM morphing winglet. .......................................... 8

Figure 1.5. Sliding panels having backward-facing steps and gaps between them to accommodate the morphing motion of a two-modules morphed winglet. Reproduced from [18]

Figure 1.6. Segmented sliding skin of a NACA 2412 with chord-wise distributed (a) backward-facing steps, and (b) trenches.

Figure 1.7. Streamlines of the velocity field at the vicinity of a backward-facing installed on the upper surface of a NACA $2412\left(\operatorname{Re}=5.7 \mathrm{e} 6, \mathrm{M}=0.17\right.$, and $\left.\alpha=2.5^{\circ}\right)$.

Figure 1.8. The structure of this thesis and its six chapters.

Figure 2.1. Predicted blade loading for the Pak-B Low-Pressure turbine at a Reynolds number of 75,000 and a freestream turbulent intensity of $2.35 \%$. Reprinted from [30].

Figure 2.2. A quadrilateral cell at $\boldsymbol{i t h}$ and $\boldsymbol{j t h}$ position with all its neighbouring cells and mesh spacing.

Figure 2.3. The isotropic scaling down method with the origin of scaling at the cell's center.

Figure 2.4. Different types of meshes that will be used to assess the order of accuracy of the three gradient operators 28

Figure 2.5. 3D representation of Ackley's testing function. 29

Figure 2.6. L1 norm convergence of solution on curved quadrilateral mesh using the three gradient reconstruction methods.

Figure 2.7. Observed order of accuracy of the reconstructed gradients, calculated using the three gradient reconstruction methods.

Figure 2.8. Three consecutively refined meshes used with (a) the NACA0012, (b) the flat plate, (c) rotating Couette flow on equilateral triangular mesh and (d) rotating Couette flow with perturbed mesh.

Figure 2.9. Geometry and computational domain of the flat plate test case.

Figure 2.10. Geometry and computational domain of outer and inner cylinder of the rotating Couette flow. 36

Figure 2.11. Convergence of the spatial truncation error in the four cases.................................... 37

Figure 2.12. Comparison of the computational expenses of the gradient operators for the four cases. 38

Figure 2.13. Comparison between the CFD and the experimental values [44] of (a) the lift coefficient $\mathrm{Cl}$ at different angle of attacks $\alpha$ and (b) the drag coefficient $\mathrm{Cd}$ at different lift coefficient $\mathrm{Cl}$ values. 
Figure 3.1. A schematic diagram of the cavity computational domain and the used nomenclature...45 Figure 3.2. Y-component of the velocity fluctuations measured in three computational domains with different sizes. 46

Figure 3.3. The blocking and mesh configurations used to resolve the flow over cavities. 47 Figure 3.4. Spatial convergence of the fundamental frequency towards the asymptotic extrapolated value as the number of elements in the mesh increases. 49

Figure 3.5. Comparison between the y-velocity oscillations in a wake mode flow on three consecutively refined meshes.

Figure 3.6. Y-velocity fluctuations over a cavity obtained using four different time-steps of $\Delta t U / D=$ $5.2,0.52,0.052,0.0052$..

Figure 3.7. Comparison between the y-velocity oscillations in the three cavity flow regimes; nooscillations, shear layer mode, and wake mode.

Figure 3.8. Intermittency contour plot of two cases in a cavity 'no-oscillations' mode. (a) shows no signs of boundary layer transition, while the cavity in (b) triggered the transition of the boundary layer.

Figure 3.9. Schematic diagram of the four steps feedback process that results in the development of the shear layer mode.

Figure 3.10. Comparison between the numerical fast Fourier transformation spectrum and the fundamental frequencies predicted by Rossiter [52] and Heller \& Bliss [74] semi-empirical equations.

Figure 3.11. Comparison between (a) Schlieren photographs obtained experimentally by Krishnamurty [51], and (b) the results obtained from the numerical model for a Mach number of 0.8, and a cavity with $\mathrm{L} / \mathrm{D}=2$. .55

Figure 3.12. Four instantaneous contour plots of the non-dimensional vorticity magnitude showing the steps of the vortex shedding and ejection in the case of a wake mode flow at approximately (a) $\mathrm{t}=\mathrm{T} / 4$, (b) $\mathrm{t}=\mathrm{T} / 2$, (c) $\mathrm{t}=3 * \mathrm{~T} / 4$, and (d) $\mathrm{t}=\mathrm{T}$, where $\mathrm{T}$ is the periodic time of a single vortex shedding cycle.

Figure 3.13. The state of the boundary layer (Laminar or Turbulent) over cavities plotted versus the non-dimensional quantity $L / \theta * \sqrt{\operatorname{Re} \theta}$. .

Figure 3.14. A $3 \mathrm{D}$ case of a wing with a trench having a variable length along the span. The transition of the boundary layer was shifted to the trench location at an $L / \theta * \sqrt{\operatorname{Re} \theta}$ value of 596.86 . 61

Figure 3.15. A $3 \mathrm{D}$ section of a morphing wing covered with sliding morphing panels. A greyscale image of figure (1.6 b).

Figure 3.16. A trench introduced on the upper surface of a NACA 2412 airfoil at $X / C=0.25$ with different widths ranging from 0.001 to 0.0075 of the chord length.

Figure 3.17. Lift coefficient $\mathrm{cl}$, drag coefficient $\mathrm{cd}$ and lift-to-drag ratio cl/cd of a NACA 2412 airfoil with trenches located at $\mathrm{X} / \mathrm{C}=0.25$ with different widths.

Figure 3.18. Contour plot of the intermittency function over the trenched airfoil. The zoom-in over the cavities show that the boundary layer maintained its laminar state. 
Figure 3.19. Contour plot of the intermittency function over the ill-designed trenched airfoil. The close-up on the cavities show that the boundary layer experienced transition from the first cavity. ..68 Figure 4.1. Streamlines and pressure coefficient distribution over an overlapping morphing skin ( $\operatorname{Re}$ $=5.7 \mathrm{e} 6, \mathrm{M}=0.2$, and $\alpha=2.5^{\circ}$ ). 70

Figure 4.2. A schematic diagram of a clean NACA 2412 (National Advisory Committee for Aeronautics) overlaying the stepped NACA 2412 airfoil.. .72

Figure 4.3. Three consecutively refined meshes used to estimate the spatial convergence...............73 Figure 4.4. Assessing the mesh spatial convergence using (a) the Richardson's extrapolation method, and (b) the logarithmic slope of the converged errors. 74

Figure 4.5. The two extreme locations of the step on the upper surface of the NACA 2412 ...........75 Figure 4.6. The variation of the lift coefficient at different step locations $(\operatorname{Re}=5.7 \mathrm{e} 6, \mathrm{M}=0.2$, and $\alpha$ $=2.5^{\circ}$ ). The dashed red lines emphasize the change in the slope of the obtained values. .76

Figure 4.7. The pressure distribution over the upper surface of stepped airfoils with different step locations as well as the pressure distribution over the clean NACA 2412 airfoil $(\operatorname{Re}=5.7 \mathrm{e} 6, \mathrm{M}=0.2$, and $\alpha=2.5^{\circ}$ ). .77

Figure 4.8. Streamlines showing the separation of the flow at the step edge and the creation of low pressure recirculation zones over the step's vicinity. The step is located at the mid-chord length on the suction side of a NACA $2412\left(\operatorname{Re}=5.7 \mathrm{e} 6, \mathrm{M}=0.2\right.$, and $\left.\alpha=2.5^{\circ}\right)$. 78

Figure 4.9. The variation of the drag coefficient at different step locations $(\operatorname{Re}=5.7 \mathrm{e} 6, \mathrm{M}=0.2$, and $\left.\alpha=2.5^{\circ}\right)$. .79

Figure 4.10. Skin friction coefficient values for airfoils with a step (a) before the viscous boundary layer transition point and (b) after the transition point $\left(\operatorname{Re}=5.7 \mathrm{e} 6, \mathrm{M}=0.2\right.$, and $\left.\alpha=2.5^{\circ}\right)$. .80

Figure 4.11. Decomposition of the drag coefficient to pressure drag and viscous drag coefficients.. 82 Figure 4.12. (a) Lift and (b) drag coefficients versus angle of attack $\alpha(\operatorname{Re}=5.7 \mathrm{e} 6$ and $M=0.2) \ldots 83$ Figure 4.13. The two extreme depths of the step. 84

Figure 4.14. Effect of the step depth on the lift coefficient of the stepped NACA 2412 airfoil $(\operatorname{Re}=$ 5.7e6, $\mathrm{M}=0.2$, and $\left.\alpha=2.5^{\circ}\right)$..

Figure 4.15. Pressure distribution over NACA 2412 airfoils with different step depths $(\operatorname{Re}=5.7 \mathrm{e} 6, \mathrm{M}$ $=0.2$, and $\alpha=2.5^{\circ}$ ). .86

Figure 4.16. Effect of the step depth on the drag coefficient of the stepped NACA 2412 airfoil $(\operatorname{Re}=$ 5.7e6, $\mathrm{M}=0.2$, and $\left.\alpha=2.5^{\circ}\right)$......

Figure 4.17. Decomposition of the Drag coefficient to pressure drag and viscous drag coefficients at different step depths. .88

Figure 4.18. Different configurations of the NACA 2412 with different step angles......................89

Figure 4.19. Convergence of the lift coefficient values as the mesh density increases. ....................91

Figure 4.20. The lift coefficient value $c l$ at different step locations $\left(\operatorname{Re}=5.7 \mathrm{e} 6, \mathrm{M}=0.2\right.$, and $\alpha=2.5^{\circ}$ ).

Figure 4.21. Streamlines of the flow around the stepped NACA 2412 showing its separation at the step edge, the creation of a recirculation zone and the further reattachment of the flow. .94 
Figure 4.22. Pressure distribution over the lower surface of a clean NACA 2412 as well as in cases of stepped airfoil with steps at different locations.

Figure 4.23. Pressure distribution over the lower surface of a clean NACA 2412 as well as in cases of stepped airfoil with steps at different locations ( $\operatorname{Re}=5.7 \mathrm{e} 6, \mathrm{M}=0.2$, and $\alpha=2.5^{\circ}$ ). 96

Figure 4.24. Decomposition of the drag coefficient to pressure drag and viscous drag coefficients $(\operatorname{Re}$ $=5.7 \mathrm{e} 6, \mathrm{M}=0.2$, and $\alpha=2.5^{\circ}$ ).

Figure 4.25. Skin friction coefficient distribution over the lower surface of a clean NACA 2412 as well as in cases of stepped airfoil with steps at different locations $\left(R e=5.9 \times 106\right.$ and $\left.\alpha=2.5^{\circ}\right) .98$ Figure 4.26. (a) Lift and (b) drag coefficients versus angle of attack $\alpha(\operatorname{Re}=5.7 \mathrm{e} 6$ and $\mathrm{M}=0.2) \ldots 99$ Figure 4.27. The lift coefficient value $\mathrm{cl}$ at different step depths $\left(\operatorname{Re}=5.7 \mathrm{e} 6, \mathrm{M}=0.2\right.$, and $\left.\alpha=2.5^{\circ}\right)$.

Figure 4.28. The drag coefficient value $c d$ at different step depths of a backward facing step installed on the lower surface of a NACA 2412 airfoil $\left(\operatorname{Re}=5.7 \mathrm{e} 6, \mathrm{M}=0.2\right.$, and $\left.\alpha=2.5^{\circ}\right)$.

Figure 4.29. Decomposition of the drag coefficient to pressure drag and viscous drag coefficients at different step depths.

Figure 4.30. Streamlines of the recirculation zone and the Moffatt eddies at different step angles. 105 Figure 5.1. Geometrical change of conventional mesh compared to zero-Poisson ratio accordion mesh.

Figure 5.2. Geometric parameters of a single element of a zero-Poisson ratio accordion mesh. ..... 109 Figure 5.3. Aluminum rods supporting the accordion mesh to minimize the out-of-plane deflection.

Figure 5.4. Relationship between the separating distance of the spars and the diameter of the supporting rods.

Figure 5.5. The morphed accordion mesh when experiencing (a) tensile loading and (b) shear loading with the accompanying Von-Misses stresses calculated using FEA.

Figure 5.6. Different shapes of the minor ribs proposed by the author to reduce the local stresses for the in-plane motions.

Figure 5.7. Maximum local strain obtained from the FEA when different shapes for the minor ribs are used. 114

Figure 5.8. (a) Highly concentrated stresses when twisting a single minor rib compared to (b) low stresses when 4 minor ribs are used instead.

Figure 5.9. Design of the composite material with its fibers parallel to the chord-wise direction. .. 116

Figure 5.10. Temperature range of different elastomer materials.

Figure 5.11. Prototype of the morphing cellular structure when morphed in the allowable degrees of freedom.

Figure 5.12. Experimental set-up to test the out-of-plane deflection of the morphing skin.

Figure 5.13. Interpolating the skin wrinkles into Gaussian distribution and adding it to the NACA 2412 profile. 
Figure 5.14. 15 configurations of the wrinkled NACA 2412 airfoil with wrinkles added at (a) $X / C=$ 0.25 , (b) $\mathrm{X} / \mathrm{C}=0.50$, and (c) $\mathrm{X} / \mathrm{C}=0.75$.

Figure 5.15. A family of three consecutively refined meshes used to apply Richardson's extrapolation method and estimate the spatial convergence.

Figure 5.16. The lift coefficient values $\mathrm{Cl}$ obtained on the coarse, medium and fine meshes, as well as the value extrapolated using Richardson's extrapolation method. 126

Figure 5.17. Lift coefficient values $\mathrm{Cl}$ of wrinkled airfoils with different wrinkle heights and locations along the chord-wise direction of the airfoil.

Figure 5.18. The pressure coefficient distribution for different wrinkles heights located at (a) $X=0.25$ C, (b) $\mathrm{X}=0.50 \mathrm{C}$ and (c) $\mathrm{X}=0.75 \mathrm{C}$.

Figure 5.19. Drag coefficient values $C d$ of wrinkled airfoils with different wrinkle heights and locations along the chord-wise direction of the airfoil. 130

Figure 5.20. The upper surface skin friction coefficient distribution for different wrinkles heights located at (a) $\mathrm{X}=0.25 \mathrm{C}$, (b) $\mathrm{X}=0.50 \mathrm{C}$ and (c) $\mathrm{X}=0.75 \mathrm{C}$.

Figure 5.21. Intermittency contour plot in case of (a) clean airfoil, (b) wrinkled airfoil with the wrinkle at $\mathrm{X} / \mathrm{C}=0.25$, (c) at $\mathrm{X} / \mathrm{C}=0.50$, and at (d) $\mathrm{X} / \mathrm{C}=0.75$.

Figure 5.22. The lift-to-drag ratio of wrinkled airfoils with different wrinkle heights and locations along the chord-wise direction of the airfoil. 134 


\section{LIST OF NOMENCLATURE}
A Reference surface area
C Airfoil chord length
D Depth of a cavity
E Young's modulus of a certain material
$\mathcal{F} \quad$ Aerodynamic property used in the Richardson's Extrapolation method
G Shear modulus of a certain material
J Moment of inertia
$\mathrm{k} \quad$ Specific turbulent kinetic energy
L/D Lift-to-drag ratio
M Freestream Mach number
$p \quad$ Observed order of accuracy of a numerical solution
$\mathrm{P} \quad$ Static pressure on the surface of an airfoil
$\mathrm{r} \quad$ Refinement ratio from one mesh level to the other
S Shape factor of the boundary layer
t Time
T Torque
$\mathrm{u} \quad \mathrm{x}$-component of the disturbance velocity
v y-component of the disturbance velocity
W Width of the computational domain
Y Height of the computational domain
$A_{f} \quad$ Surface vector
$c_{d} \quad$ Drag coefficient
$c_{l} \quad$ Lift coefficient
$c_{r} \quad$ Propagation velocity of the disturbance wave in the x-direction
$C_{f} \quad$ Skin friction coefficient
$D_{L} \quad$ Depth of a step on the lower surface of an airfoil 


$\begin{array}{cl}D_{U} & \text { Depth of a step on the upper surface of an airfoil } \\ f_{n} & n^{t h} \text { mode frequency } \\ L_{U} & \text { Length of a rectangular cavity located on the upper surface of an airfoil } \\ \hat{n} & \text { A unit vector normal to the surface } \\ N_{f v} & \text { Number of nodes defining the face } \\ P_{0} & \text { Reference pressure } \\ R e & \text { Reynolds number } \\ R e_{X} & \text { Reynolds number based on length } \\ R e_{\theta} & \text { Reynolds number based on boundary layer momentum thickness } \\ S t_{n} & \text { Strouhal number of the } n^{\text {th }} \text { mode frequency } \\ \hat{t} & \text { Unit vector tangent to the airfoil surface } \\ X_{U} & \text { Location of a step or a cavity on the upper surface of an airfoil } \\ w_{i} & \quad \text { Weighting function used to calculate the contribution of each cell to the } \\ & \text { gradient calculation }\end{array}$

\title{
Greek Letters and Mathematical Symbols:
}

\author{
$\forall \quad$ Control volume \\ $\nabla \quad$ Gradient operator \\ $\alpha \quad$ Flow angle of attack /A real number related to the disturbance wave length \\ $\beta \quad$ Circular frequency of flow disturbance \\ $\theta \quad$ Boundary layer momentum thickness \\ $\gamma \quad$ Intermittency function / Specific heat ratio \\ $\delta \quad$ Boundary layer thickness \\ $\varepsilon \quad$ solution \\ $\kappa \quad$ Empirical constant related to the convection speed of the disturbance
}




$\begin{array}{ll}\lambda & \text { Flow disturbance wave length } \\ \rho & \text { Density of the fluid } \\ \psi & \quad \text { Instantaneous stream function of the perturbation velocity } \\ \omega & \text { Specific dissipation rate } \\ \phi & \text { Amplitude function of the perturbation / A scalar quantity } \\ v & \text { Poisson ratio } \\ \alpha_{c r} & \text { Critical angle of attack } \\ \beta_{i} & \text { Amplification factor of flow disturbance } \\ \delta^{*} & \text { Boundary layer displacement thickness } \\ \lambda_{x} & \text { Lagrange multipliers a linear function in the x-direction } \\ \lambda_{y} & \text { Lagrange multipliers a linear function in the y-direction } \\ \tau_{w} & \text { Wall shear stresses } \\ \omega_{i} & \text { Angular velocity } \\ \phi_{N_{i}} & \text { Nodal value of a scalar quantity at the } i^{t h} \text { node }\end{array}$




\section{List OF ABbreVIATIONS AND ACRONYMS}

$\begin{array}{cl}\text { CFD } & \text { Computational Fluid Dynamics } \\ \text { CPU } & \text { Central processing unit } \\ \text { DNS } & \text { Direct Numerical Simulations } \\ \text { FEA } & \text { Finite element analysis } \\ \text { GGCB } & \text { Green Gauss cell-based gradient reconstruction method } \\ \text { GGNB } & \text { Green Gauss node-based gradient reconstruction method } \\ \text { LSCB } & \text { Least squares cell-based gradient reconstruction method } \\ \text { NACA } & \text { National Advisory Committee for Aeronautics } \\ \text { NASA } & \text { National Aeronautics and Space Administration } \\ \text { NREL } & \text { National Renewable Energy Laboratory } \\ \text { SMA } & \text { Shape memory alloys } \\ \text { URANS } & \text { Unsteady Reynolds Averaged Navier Stokes } \\ \text { H.O.T. } & \text { Higher order terms } \\ \text { OOA } & \text { Observed order of accuracy } \\ \text { SST } & \text { Shear Stress Transport }\end{array}$




\section{INTRODUCTION}

\subsection{Aircraft Morphing Technology:}

It was on December 17, 1903, when Oliver Wright was sitting inside a heavier-than-air power manned machine, and his brother, Wilbur Wright, was running beside their flyer, ensuring that the wing tips will not tilt and hit the ground. For approximately 12 seconds, Oliver cruised the air at a speed of $13.4 \mathrm{~m} / \mathrm{s}$, marking the most important event in aviation history. The Wright brothers used a system of pulleys and cables to twist the angle of the trailing edges of their wings. Through this morphing mechanism, they were able to control the rolling motion of their flying machine.

Since then, aircraft technology has witnessed giant leaps forward improving different aspects of these flying machines; their aerodynamics performance, the materials used in their manufacturing, and their flight capabilities and efficiency. To maintain the structural integrity of these magnificent machines, aircraft designers preferred to use rigid structure equipped with few discrete control surfaces. Such design enables the aircraft to have optimum performance over a small range of flight conditions and have a poor performance at other flight regimes. But thanks to the recent advancement in smart material and adaptive structures, the biomimetic concept used by the Wright brothers has been revived, morphing aircraft.

'Morphing' is derived from the Latin word 'Metamorphosis' which means a major change in the appearance or character of someone or something [1]. When it comes to morphing while flying, nothing can out perform the wing morphing experts, birds. In a fluent and intrinsic manner, birds morph their wings, necks and tails to respond to external fluid forces allowing them to achieve their best aerodynamic performance for different purposes. Through morphing their bodies, birds become capable of migrating long distances by efficient cruising, attacking prey at high speeds, performing special maneuvers while escaping their predators, and performing perfect and precise landing on twigs and branches. All is done at minimum energy expenditure which is translated in aircraft to fuel efficiency, lower operating cost and increasing efficiency and versatility $[2,3]$.

Achievements in morphing aircraft technology have the potential to play vital and essential roles

in the aviation development because of the exceptional benefits they provide to aircraft. These benefits include - but are not limited to - the following: 


\section{- Improving aircraft performance to expand its flight envelope:}

While a traditional aircraft is designed to perform optimally at a single flight condition (usually cruising for commercial aircraft), and compromise its performance at other phases of its flight profile, a morphing aircraft can perform near optimum at different flight regimes. One of the studies that reflects the superiority of morphing technology over rigid aircraft designs was presented by Joshi et al. [4]. A spider plot was used to compare the aerodynamic performance of a fixed-geometry Firebee, a morphing airfoil Firebee and a morphing planform Firebee, at different flight regimes. Firbee is one of the most widely used jet-propelled target drones. Figure (1.1) shows the results of this qualitative comparison.

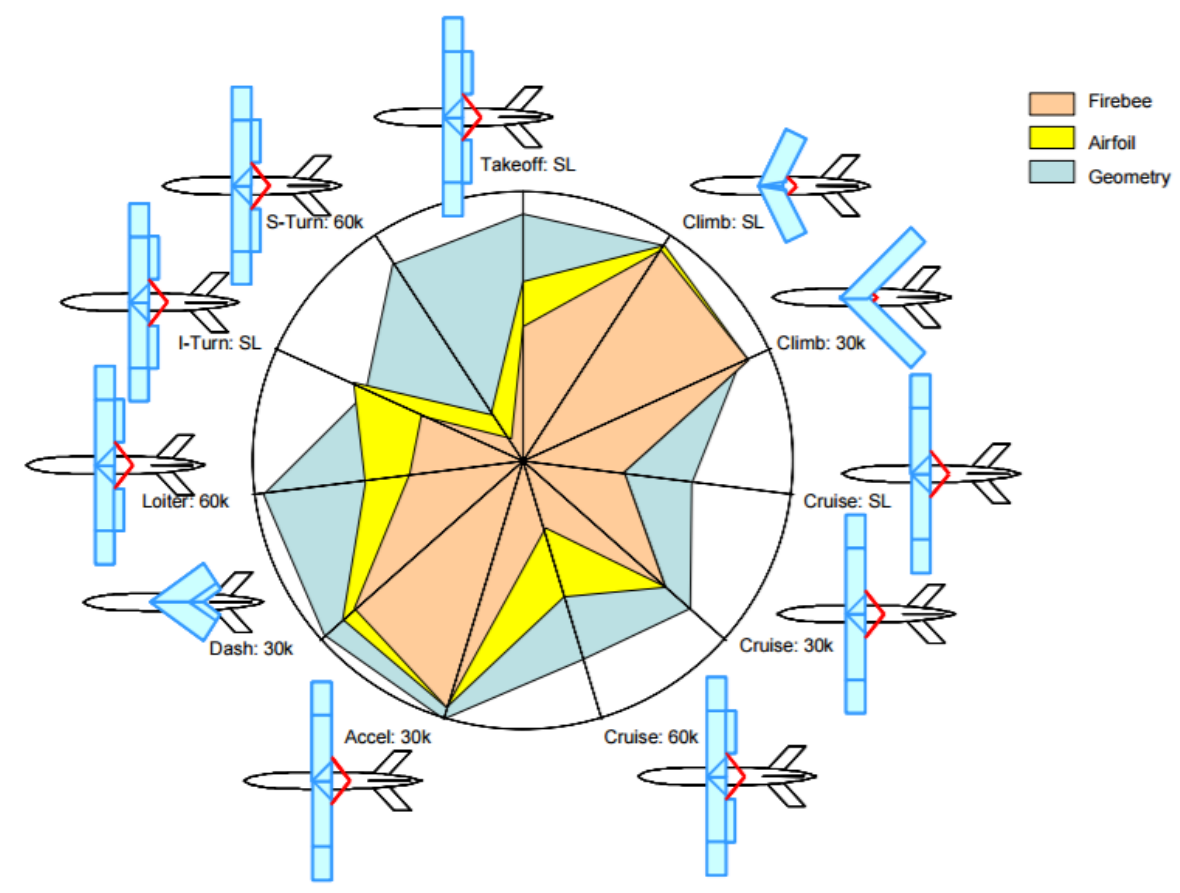

Figure 1.1. Spider plot comparing the performance of a fixed-geometry Firebee, a morphing airfoil Firebee and a morphing planform Firebee. Reproduced from [4].

The plot consists of radial lines, each representing the performance of the Firebee at various flight phases. The plot is designed such that minimum performance found at the center of the plot, and as the performance of the Firebee improves, its relative score along the radial lines increases in the outward direction. On the plot, the inner most area shown is the performance of a fixed-wing Firebee. Moving radially outward on the plot, the performance of a Firebee with morphing airfoil is highlighted in yellow. The morphing airfoil design showed noticeable improvements in most of the flight phases over the fixed wing Firebee. The most outward shaded area on the plot represents the performance of a Firebee with a morphing planform. This design can change the span of the wing, the chord length, and the sweep angle. The aerodynamic performance of the morphing 
platform Firebee surpassed the other two designs at all flight regimes. This shows how a morphing wing aircraft can have a better aerodynamic performance at different flight regimes.

\section{- Reducing fuel consumption for more affordable flights:}

As fuel cost increases, fuel-saving methods have become a major concern for many airlines and companies. Bolonkin et al. [5] estimated that $50 \%$ of the airlines operating expenses for widebody, long-range transport is related to the fuel consumption. In the same study that was conducted at NASA Dryden flight center [5], it was estimated that a 3\% reduction in the fuel consumption of an aircraft can save up to $\$ 300,000$ per year. This $3 \%$ reduction is quite achievable using morphing technology. Lyu and Martins [6] checked the effect of using a morphing trailing-edge on a Boeing 777-LR, and showed that a $1 \%$ drag reduction can be achieved at on-design conditions and a drag reduction of $5 \%$ resulted at off-design conditions. By using aerodynamics shape optimization on a hypothetical regional class aircraft, Curiale and Zingg [7] showed that full morphing of the wing can produce $2 \%$ improvement in average aircraft performance over a range of cruise conditions. While trailing-edge only morphing produced approximately $1 \%$ improvement in the overall performance.

\section{- Reducing weight for lower manufacturing costs:}

Using a genetic optimization algorithm, Roth et al. [8] examined how a variable geometry wing can morph in flight to have optimal performance. Their analyses showed that the variable wing aircraft can have $8 \%$ lower take-off gross weight and lower engine thrust requirements when compared to its fixed-wing rival. This is because approximately half the wing's mass of transport aircraft is a result of the high-lift systems complexity [9].

\section{- Solving aerodynamic problems:}

For some fighters it is a requirement to make short take-off and landing on aircraft carriers at relatively low speeds. This can be better achieved using small sweep angles of wings. At the same time, backward-swept wings are among the requirements of transonic fighters due to their proven capability of delaying compressibility problems and the onset of shockwaves [10]. For that reason, the F-14 Tomcat and the Panavia Tornado are equipped with variable sweep wings which can provide good performance at both spectrum ends of speed.

\section{- Solving technical issues:}


Morphing structures provided solutions to many technical problems in aviation. For example, the Concorde's drooped nose solved a serious problem that was faced by its trained pilots. During take-off and landing of a delta wing aircraft like the Concorde, a large angle of attack must be achieved, which in combination with the long-pointed nose of the aircraft, resulted in a total obstruction of the pilot's view. For that reason, the Concorde's engineering designers used a morphing mechanism that can change the angle of its nose. This allowed the Concorde to switch between being streamlined to reduce drag and achieve optimum aerodynamic efficiency during the flight, and not obstructing the pilot's view during taxi, takeoff, and landing operations.

Another popular morphing motion is the folding-wings design whose importance was revealed in the 1910's when Frederick Handley Page invented the World War I Handley Page O/400 Bomber. The wings of the biplane British bomber spanned a distance of $100 \mathrm{ft}$., preventing it from being stored in the popular canvas Bessoneau hangar whose width was limited to $65.5 \mathrm{ft}$ only. With the aid of the folding-wing morphing motion, the Handley Page O/400 Bomber fit inside the hangar despite its large wing size. Later in 1920, the Grumman's designing engineers followed the same concept and developed foldable wings for the Grumman Wildcat aircraft, known as the STO-wing. Their innovative design allowed the U.S. Navy to nearly double the capacity of Grumann aircraft on aircraft carriers during World War II [11].

\section{- Achieve greener and cleaner air transportation:}

With the wide and strong growth of air transportation, more stiff standards are set by the International Civil Aviation Organization (ICAO) to ensure lower emissions of $\mathrm{CO}_{2}$ and $\mathrm{NO}_{\mathrm{X}}$ by aircraft engines and lower noise emitted from the interaction of the air with the airframe discontinuities. Such standards impose additional motivation to divert from standard designs towards morphing technology where significant benefits can be realized.

For all these reasons, aircraft morphing technologies have attracted the attention of researchers, designers and manufacturers. Being the most influential parts on the aerodynamic performance of aircraft, wings have been the focus of a large portion of morphing developments. Any minor changes in the geometry of the wings, such as a couple of dihedral angles, or few sweep angles, a small change in the span or the chord length of the wing can dramatically affect the performance of the aircraft. Recent advancements in smart materials and structure technologies have allowed, not only minor geometric changes, but also large-scale morphing where every aspect of the wing's geometry can be altered.

Sofla et al. [12] classified wing morphing motions into three major types: planform alternation, out-of-plane transformation, and airfoil adjustment as shown in figure (1.2). 
The most common forms of wing planform morphing are changing span, changing chord length and changing sweep angle. The span is usually changed using a telescopic design of the wing where sections of the wing are fitted within each other with minimum clearances, and slide against each other resulting in a changing span. This mechanism is limited to designs with wings of unity taper ratio. The second form of planform morphing is changing chord length. This is usually achieved in conventional aircraft through the deployment of slats and flaps during take-off and landing, however, recent studies are focusing on achieving similar geometric alteration but through seamless surface. Changing the sweep angles of the wings are usually achieved through pivoting mechanisms that are usually complicated and heavy, but reliable. This concept was successfully implemented in many military aircraft such as: Bell-X-5, F-111, F-14, and B-1 for its proven aerodynamic benefits.

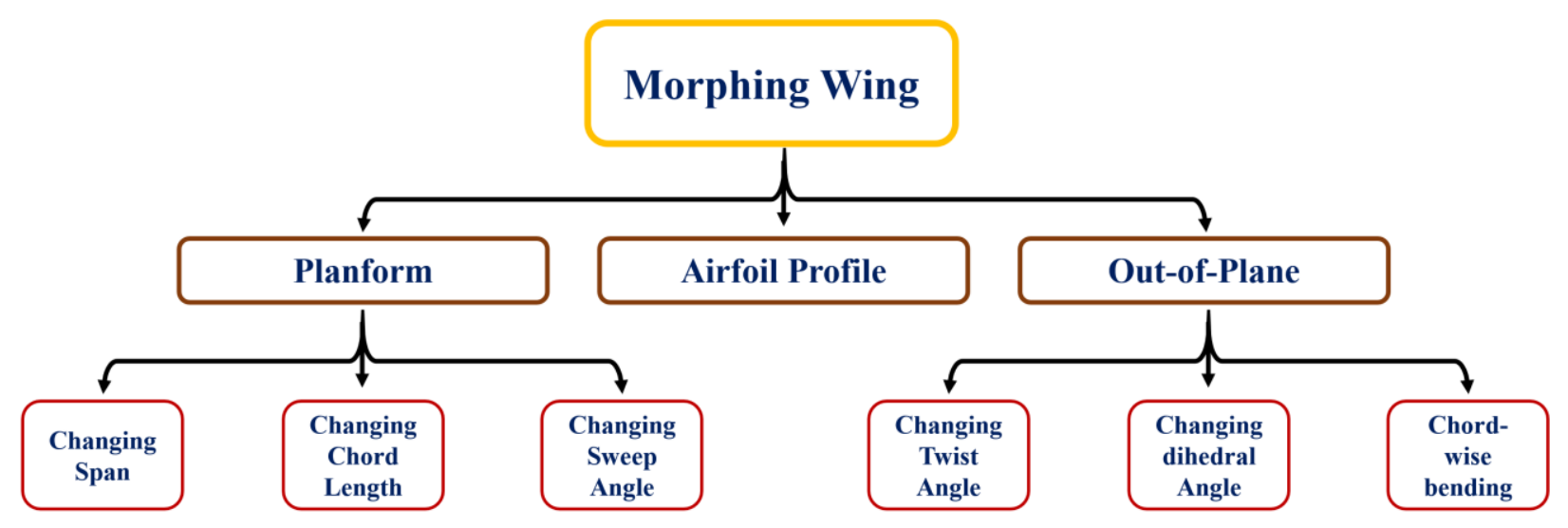

Figure 1.2. Classification of common morphing motions of wings as classified by Sofla et al. [12].

The second category of morphing wing motion is airfoil profile alternation. This concept has recently attracted a lot of numerical and experimental efforts due to its relatively simple and proven concepts that showed significant improvements in the airfoil performance.

The third concept of morphing technology is the out-of-plane motion which is divided into three categories; Twist angle, dihedral angle and chord-wise bending. The chord-wise bending, or in other words, changing camber is the most studied morphing motion. When the camber change along the span-wise direction is constant, the motion is known as chord-wise bending. While when the camber change is non-uniform along the span-wise direction, the motion is known as twist morphing motion. The third out-of-plane motion is the dihedral angle, which imposes a challenge in its implementation because this motion is usually along the axis of the major aerodynamic force; the lifting force. Recent studies in wings morphing technology is trying to mix between some of the aforementioned morphing motions. 


\subsection{Morphing Winglet Project:}

At the Department of Aerospace Engineering at Ryerson University, the morphing wing research team is studying the development of a modular morphing wing. The wing consists of several morphing modules, each possesses four different degrees of freedom. These motions are changing the span of the wing, changing the sweep angle, the twist angle and the cant (dihedral) angle of the module. This is achieved through a variable geometry truss mechanism (VGTM) that is fitted within the wing envelope.

This design can withstand all the aerodynamic and mechanical loads such as shear, torsion, bending, tension, compression, etc. through its self-locking mechanism. Figure (1.3) shows the morphing structure achieving its four aforementioned motions.

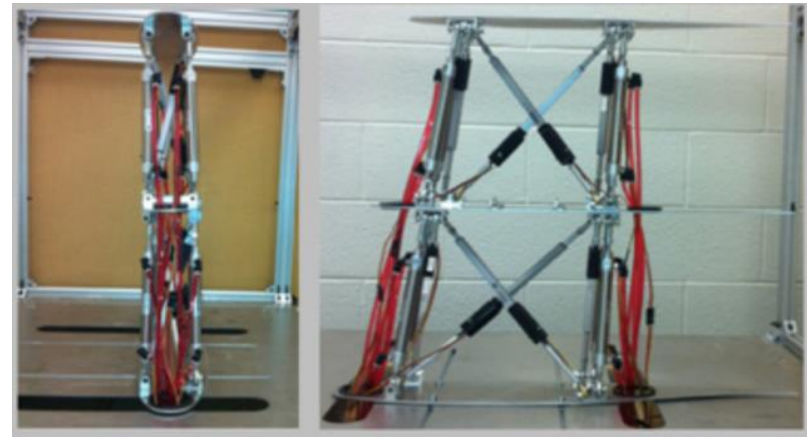

(a) Span

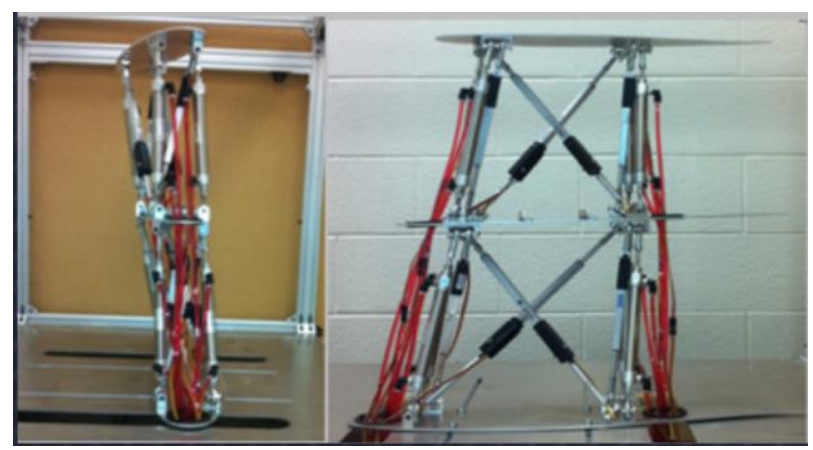

(c) Twist

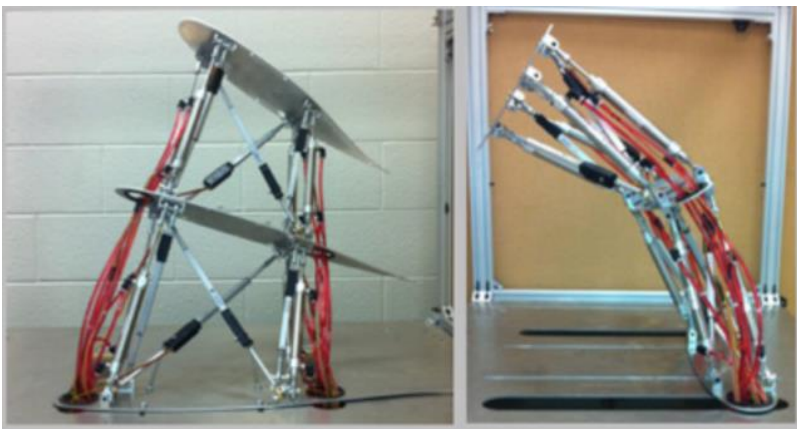

(b) Sweep

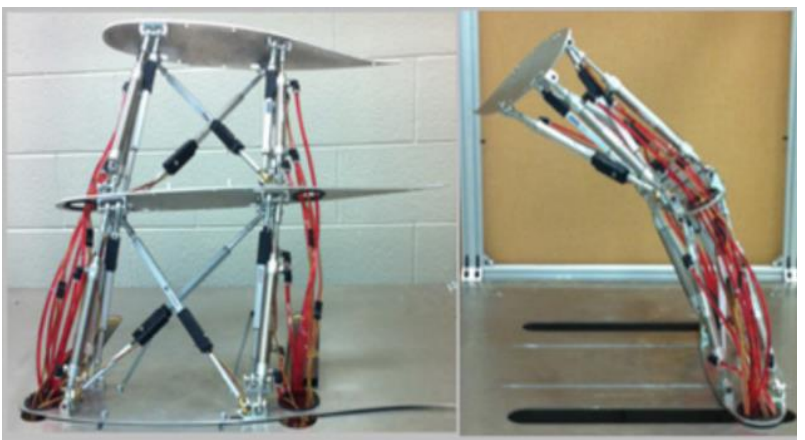

(d) Cant

Figure 1.3. A four degrees-of-freedom morphing wing using variable geometry truss mechanism to achieve (a) span, (b) sweep, (c) twist and (d) cant motions. Reprinted from [13]. The morphing wing module shown in figure (1.3) uses the under-actuation theory where the number of actuators are less than the achieved degrees of freedom. Each one of the modules 
consists of a base and a platform linked with four active linear actuators, and four passive linear links with a self-locking mechanism. Several modules have been designed tested and assembled together to form the morphing wing.

As a subset of the morphing wing project, our research team current focus is developing a 2 module VGTM winglet for a Bombardier Inc. regional jet aircraft. The exact model of the aircraft was not provided by Bombardier Inc., the only information provided was the chord length of wing tip which is $\sim 1 \mathrm{~m}$. A draft geometry of the wing was provided by Bombardier Inc. as shown in figure (1.4). The root chord length was back engineered and measured to be $3.5 \mathrm{~m}$. This makes the mean chord length of the wing approximately $1.75 \mathrm{~m}$. For an average Reynolds number of 10 million for regional jets calculated at the mean chord length, the Reynolds number at the root of the wing will be approximately 5.7 million. For that reason, most of the investigations in this thesis are done at a Reynolds number of 5.7 million.

The morphing winglet will be fixed at end of a fixed wing as shown in figure (1.4). The required morphing motion is achieved thorough 2 modules of VGTM that are installed between the fixed wing and the morphing winglet. This morphing winglet emulates many of the features of the desired morphing wing, including improved aerodynamics and reduced fuel consumptions. If this design fulfilled its objectives, the development of a VGTM fully morphing wings will be pursued. 


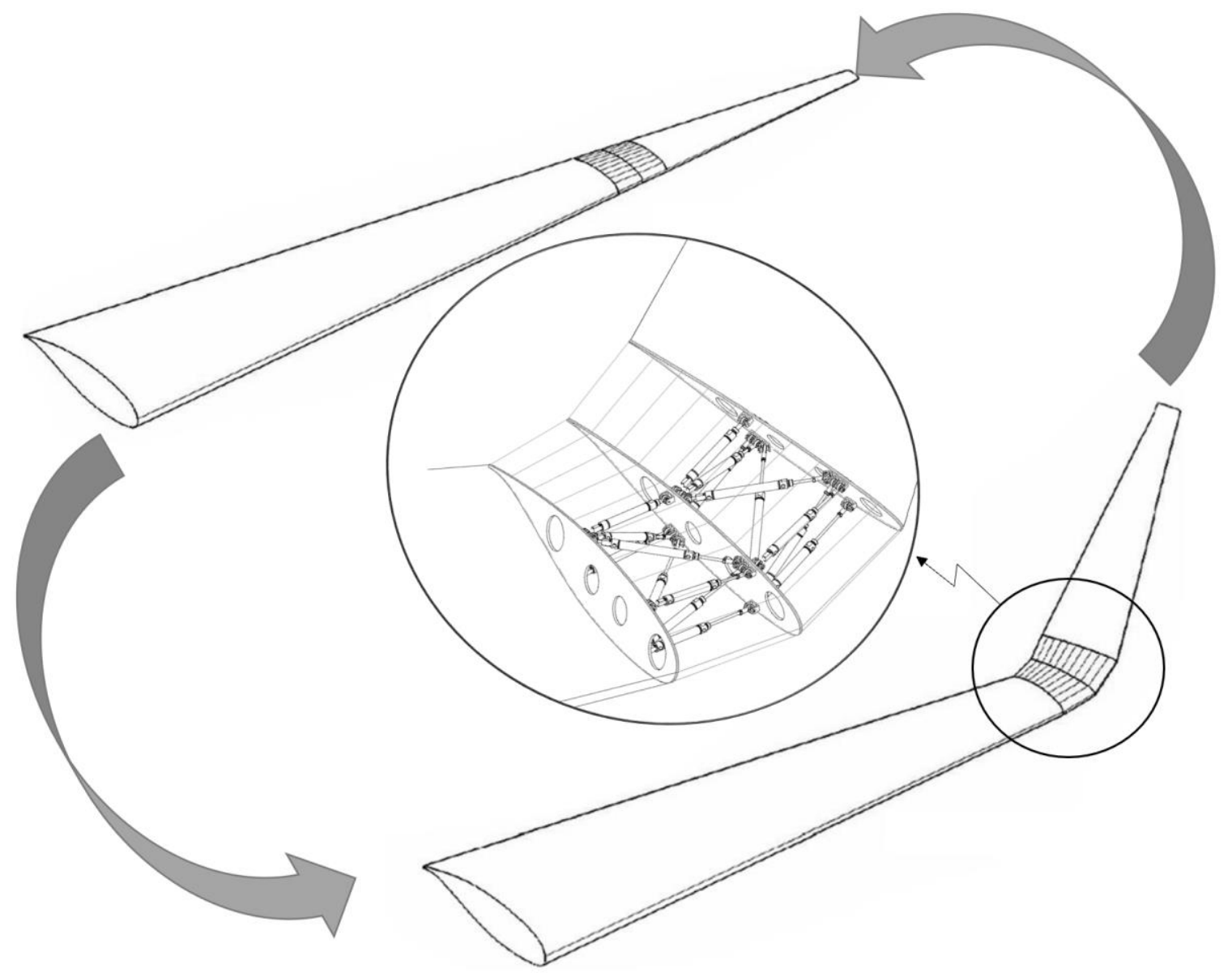

Figure 1.4. Proposed design for a 2 module VGTM morphing winglet.

This morphing technology is still facing major challenges that hinder its level of maturity. However, the most demanding of them all is developing a reliable morphing skin that can accommodate all the required morphing motions, at the same time be stiff enough to carry and transfer the gravitational and aerodynamic loads. Kikuta [14] outlined the requirements of a morphing skin well; elastic/flexible in the direction of morphing to allow low force actuation, stiff enough to withstand aerodynamic and inertial loads, abrasion and chemical resistant, resistant to different weather conditions, high strain capability, high strain recovery rate and environmental longevity and fatigue resistance. With all these diverse requirements of a morphing skin, very few designs can be considered for the task. Thill et al. [15] presented a comprehensive review of several contemporary morphing skin technologies. Among all designs, two groups of skins seemed to be promising and fulfilled most of the aforementioned requirements. These two categories are the segmented sliding skin and the flexible (stretchable) skin.

At the department of Aerospace Engineering at Ryerson, the morphing wing research team has pursued the sliding panels design. Segmented sliding skin consists of several rigid panels that constitute the outer shell of the wing surface and change their orientations during morphing based 
on the kinematics of the morphing wing. Each module is covered with a telescopic sliding panel laterally interlayered with adjacent panels. The kinematics of these panels were solved in [16-18] to show that this design is kinematically a viable solution. Depending on the required configurations, the panels can either have backward-facing steps between them, rectangular gaps, or both as shown in figure (1.5).

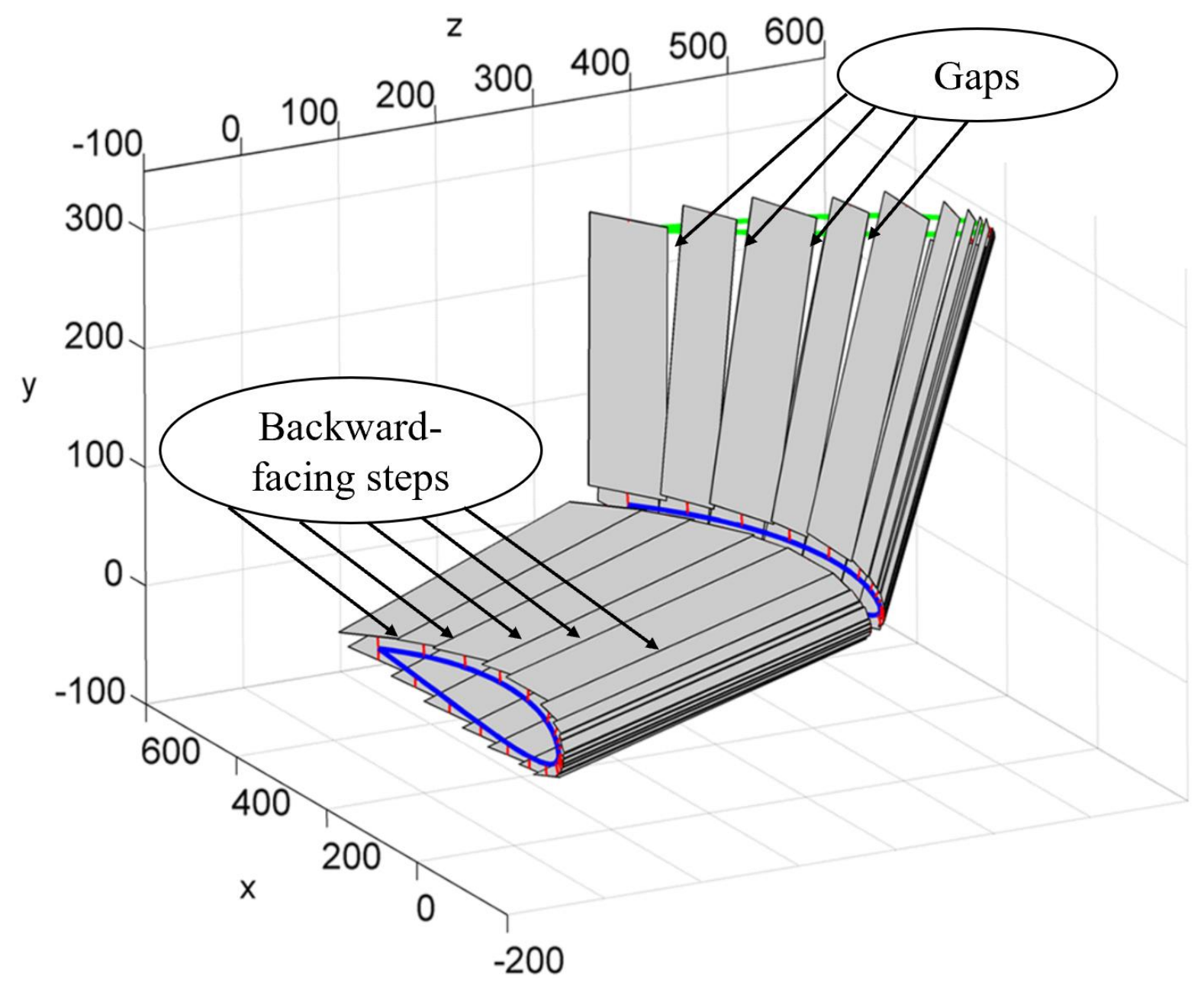

Figure 1.5. Sliding panels having backward-facing steps and gaps between them to accommodate the morphing motion of a two-modules morphed winglet. Reproduced from [18].

When the panels are overlapping, they are regarded from the aerodynamics perspective, as a cascade of backward-facing steps employed over the surface of the morphing wing. A simplified case of these backward-facing steps is shown in figure (1.6 a). When the panels are separated with small gaps, the segmented skin is treated as a wing with several trenches employed along the chord-wise direction of the wing as shown in figure (1.6 b). Despite the large similarity between 
the two designs, their aerodynamic performance is quite different in terms of their pressure distribution, wall shear stress distribution, and their influence on the boundary layer characteristics.

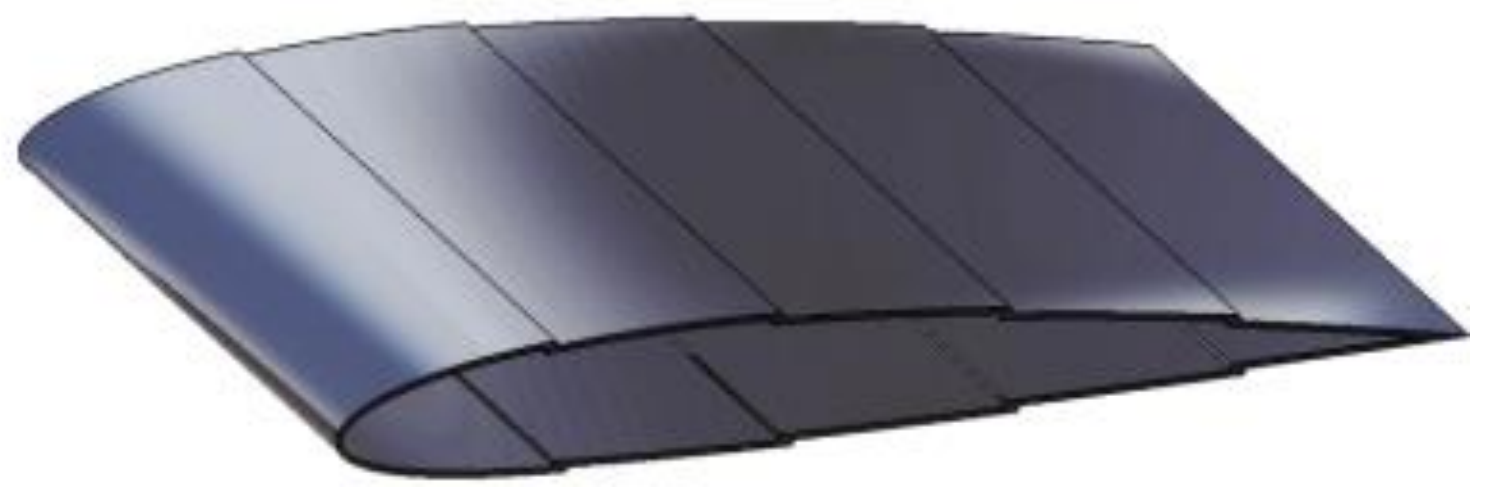

(a)

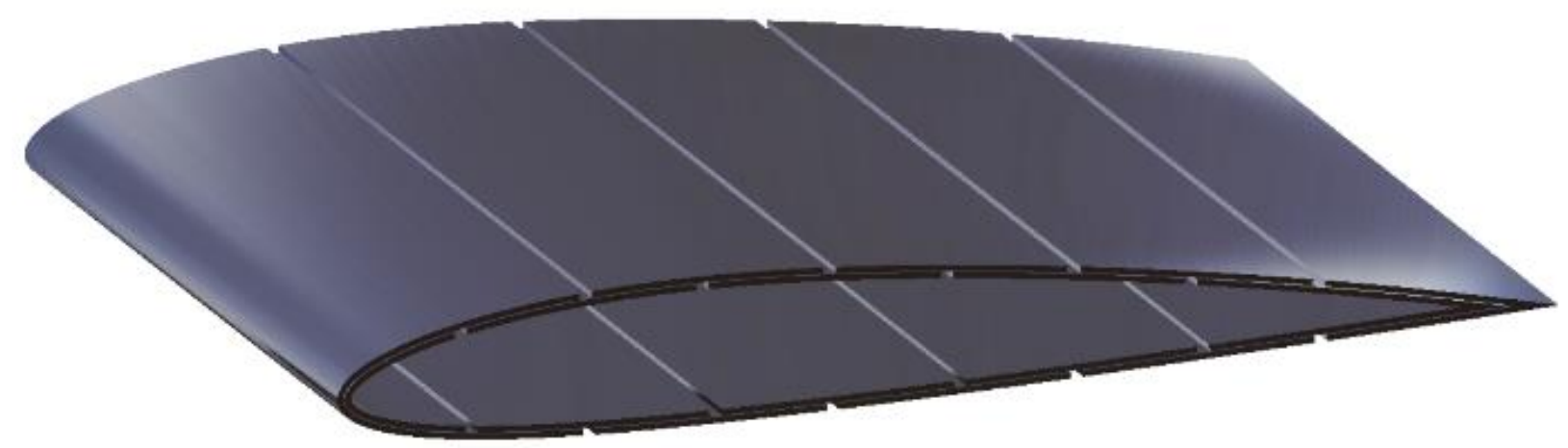

(b)

Figure 1.6. Segmented sliding skin of a NACA 2412 with chord-wise distributed (a) backwardfacing steps, and (b) trenches.

In the case of backward-facing steps, the steps extend along the span of the wing and are distributed at specific intervals along the chord-wise direction of the wing. Starting from the leading edge of the wing, the boundary layer develops gradually as the flow travels over the wing, when suddenly the flow is interrupted with the leading sharp edge of the step and separates from the surface. Figure (1.7) shows the streamlines of the velocity field for a backward-facing step installed on the upper surface of a NACA 2412 airfoil at the mid-chord location.

The separation of the flow at the upper edge of the step creates a low-pressure recirculation zone at the step vicinity that attracts the boundary layer back to the wing's surface. This alters the aerodynamic properties of the flow over the step. A more serious problem associated with backward-facing steps is that they introduce a recirculation zone with inflected velocity profiles. 
These inflectional velocity profiles are intrinsically unstable and will result in the transition of the boundary layer, no matter how small this step is.

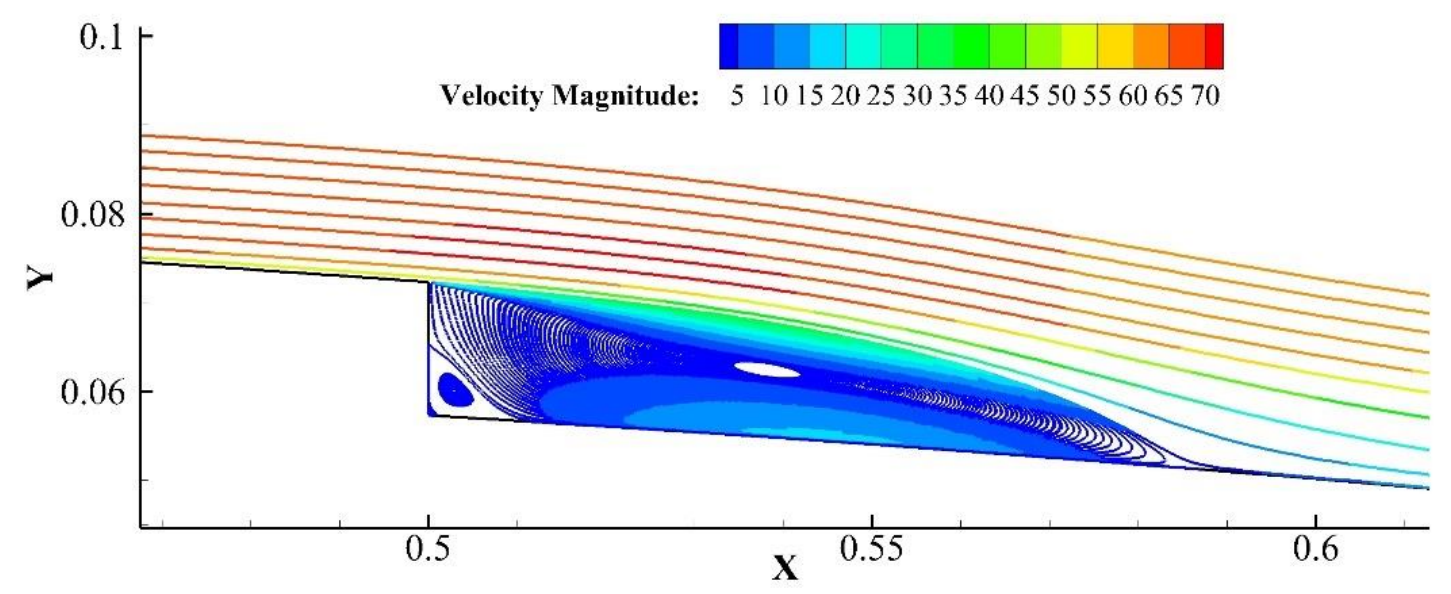

Figure 1.7. Streamlines of the velocity field at the vicinity of a backward-facing installed on the upper surface of a NACA $2412\left(\operatorname{Re}=5.7 \mathrm{e} 6, \mathrm{M}=0.17\right.$, and $\left.\alpha=2.5^{\circ}\right)$.

The intrinsic instability of inflectional velocity profiles can be derived using the Orr-Sommerfeld linear stability analysis developed by William McFadden Orr [19, 20] and Arnold Sommerfeld [21] at the beginning of the $20^{\text {th }}$ century. The analysis proved that the inflected velocity profile that develops over backward-facing step is intrinsically unstable and is an enough condition for instability.

Another important aerodynamic aspect that is altered by introducing discontinuities to the wing's surface is the near stall behavior. Most airfoils are designed such that they have a near optimum performance at the onset of separation [23]. Near this critical angle of attack, the flow starts to experience a strong viscid-inviscid interaction that dictates the performance of the airfoil. Introducing a backward-facing step on an airfoil, and specially on the upper surface will significantly affect the near stall behavior promoting a smaller critical angle of attack and early flow separation that will be shown later in chapter 4 .

These adverse aerodynamic effects can be avoided if gaps are found between the panels as shown in figure $(1.6 \mathrm{~b})$. The discontinuities between each two panels are regarded as trenches or rectangular cavities that are distributed along the chord-wise direction and extend along the span of each segment of the morphing wing. Unlike the backward-facing step where the boundary layer instability is unconditional at the step vicinity, trenches are conditionally stable. As the flow travels over the leading edge of the trench, the flow separates and a recirculation zone is generated at the cavity. The recirculation zone creates an inflected velocity profile which is a source of instability and may trigger the transition of the boundary layer. However, if the trailing edge of the cavity is 
'close enough', the inflected velocity profile is trimmed and the flow reattaches to the airfoil surface. By running a number of numerical test cases, it was observed that at small trenches widths, the laminar boundary layer bypasses the trench without experiencing any transition.

Designing a trench that preserves the laminar state of the boundary layer is a challenging task that requires a careful study of the primary and secondary flow features over the cavity. Despite its very simple geometry, flow over cavities is very complex to model. It consists of rich flow phenomena involving shear layers, shock waves, expansion waves and acoustic resonance, secondary flows, and entrapped vortices. Even the shear layer itself was found from experimental and direct numerical simulation (DNS) results to behave in one of three different modes. Depending on the geometry of the cavity and the flow properties, the shear layer can either be in the no oscillations mode, the shear layer mode, aka Rossiter mode, or the wake mode. Each mode has to be studied and modeled with high fidelity to derive a scaling law that can predict the critical width of the trench that will trigger the boundary layer transition.

In order to avoid dealing with discontinuities in whole, the author will propose a design of a stretchable/flexible skin in chapter 5. Several studies [24 - 26] mentioned elastomers as a good candidate for a stretchable morphing skin. Due to their very low tensile modulus, elastomers can elastically stretch up to $1000 \%$ of their original length and return back to their shape upon unloading without any plastic deformation. However, elastomers on their own are unsuitable to carry and transfer large aerodynamic loads. For that reason, an underlaying structure must be used to support flexible elastomer. A proposed design for an underlaying structure is auxetic materials. Auxetic materials have negative Poisson's ratio which become wider when stretched and narrower when compressed. This is achieved by their unique multiple cells structure with re-entrant edges that unfold upon the application of any stretching force. Such auxetic materials are characterized by their high out-of-plane stiffness and high in-plane flexibility, which make them suitable to be used as an underlying supporting structure for morphing skins. Olympio et al. [27] used a zeroPoisson ratio cellular structures as an underlying support for the morphing skin, and a similar design was tested in [26] where a high strain-to-failure silicone skin was supported by an underlying flexible honeycomb core on the morphing wing of an unmanned combat air vehicle (UCAV). In chapter 5, the author will combine these efforts in one new design a flexible morphing skin. The design consists of a compliant reinforced cellular structure covered with an elastomer composite. During morphing, stretchable skins developed small anisotropic ripples parallel to the dominant morphing degree of freedom. The presence of such ripples and wrinkles can have a noticeable impact on the aerodynamic performance of the morphing wing. The aerodynamics of such wrinkles will be studied. 


\subsection{Thesis Structure:}

The goal of this project is to numerically study the aerodynamic effects accompanied with sliding and stretchable morphing skins and reach design guidelines that can ensure an optimum aerodynamic performance.

In Chapter 2, the numerical tool used to carry out the flow simulations in the thesis will be presented. This tool is the high fidelity CFD code FLUENT V15, which is a finite volume method that solves the fluid mechanics governing equations (Navier-Stokes equations) with any additional transport equations for turbulence modeling. The turbulence model used is this thesis is the transition SST turbulence model [28 - 30]. The turbulence model will be briefly introduced with references to validation test cases that shows its reliability in modeling turbulence flow and transition of attached and shear flows. Due to a lack of resources about the accuracy of gradient operators, large portion of chapter 2 is dedicated to studying commonly used gradient reconstruction methods and their effect on the accuracy and efficiency of the CFD solution when used with different grid types. A final section in chapter 2 is dedicated to present a general validation test case for the flow over a NACA 2412, the airfoil used through out this thesis. The numerical results are compared to the experimental ones provide the reader with the required confidence in the CFD tool used and its settings. After having the CFD tool set and validated, examination of different types of morphing skins will be presented. Figure (1.8) shows a visual representation of this thesis structure.

Chapter 3 will present a detailed study on the aerodynamic performance of airfoils with sliding panels separated with gaps and rectangular cavities. As the flow travels over the cavity, it experiences a sequence of complex stages; flow separation, reattachment, acoustic radiations, hydrodynamic resonance, and amplified instabilities. To add to the complexity of these types of flows, it was observed that based on the flow geometry and flow properties, the flow over the cavity vicinity can behave in one of three different modes; no-oscillations mode, shear-layer mode, and wake mode. In chapter 3, the three modes will be studied and modeled numerically. Results from the numerical simulations will be validated against experimental, and semi-empirical results. 


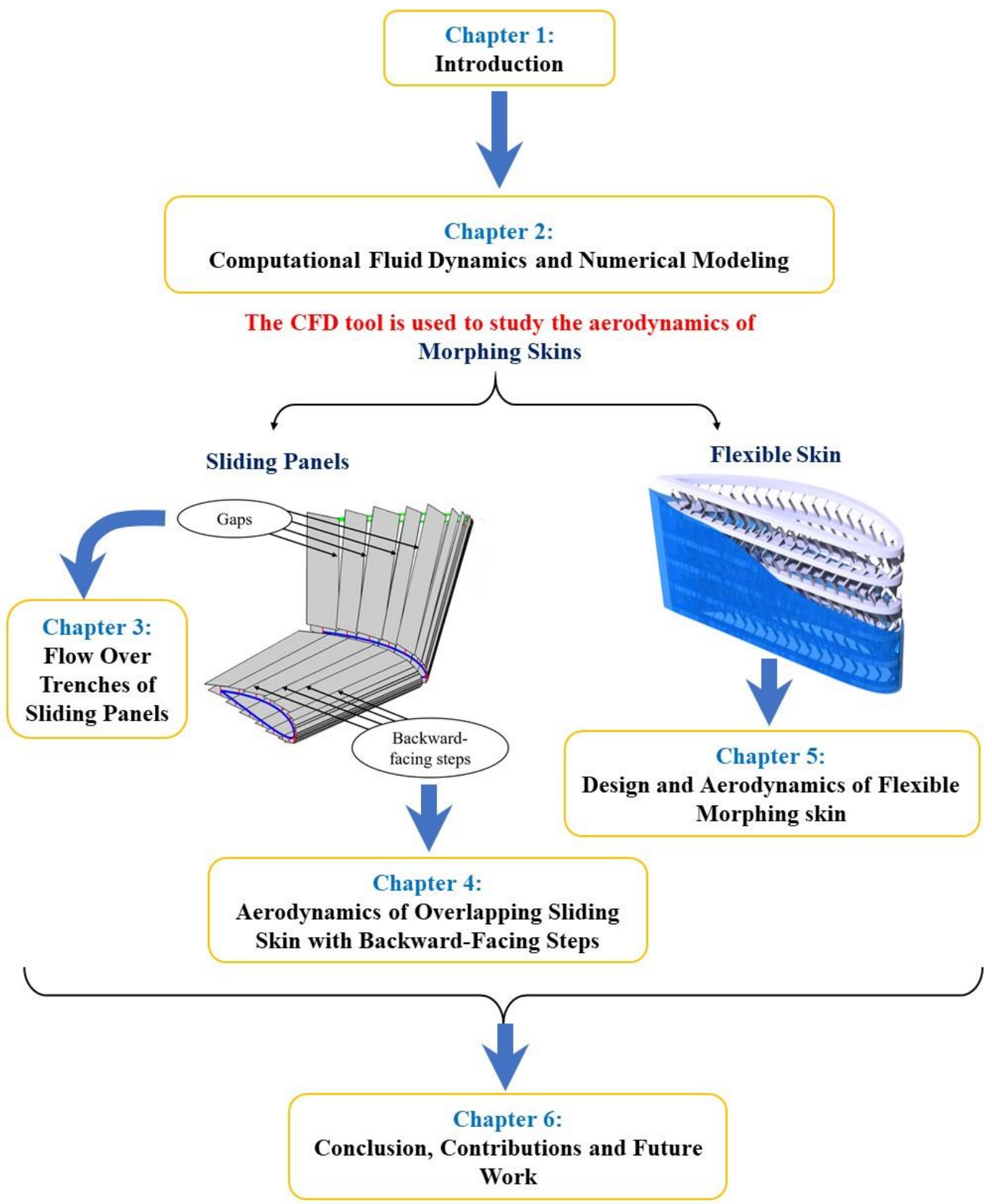

Figure 1.8. The structure of this thesis and its six chapters. 
It was observed from the numerical results that there are cases where the laminar boundary layer by-passes the trench, and other cases experience a boundary layer transition from laminar to turbulent. A separate study of the factors that trigger the transition of the boundary layer will be presented in chapter 3 , and a scaling law that can predict the critical width of the trench that triggers the boundary layer transition will be established.

Chapter 4 will focus on the aerodynamics of morphing sliding skins with backward-facing steps. In this design, the sliding panels are ordered sequentially where each panel is laying on top of the preceding panel. This creates a cascade of backward-facing steps on the upper and lower surfaces of the morphing wing. Chapter 4 will focus on studying the aerodynamic performance of airfoils with backward-facing steps employed on either side. A comprehensive numerical study will investigate the effect of the step location, depth and angle on the aerodynamic properties of the airfoil, namely, the lift coefficient $c_{l}$, the drag coefficient $c_{d}$, the lift-to-drag ratio $L / D$, and the critical angle of attack $\alpha_{c r}$.

In chapter 5, a proposed structural design for stretchable morphing skins will be studied, optimized and tested. The design consists of an underlaying flexible structure used for the reinforcement of the structure and to carry the aerodynamic loads. This structure is covered with a highly anisotropic composite material that has been tailored to be flexible in certain degrees of freedom, and very strong in other directions. This design will be studied, optimized, and a prototype will be manufactured and experimentally tested to show its capability of carrying outof-plane loads. In the same chapter, the aerodynamics of wrinkled airfoils will be studied to examine the adverse effect that a flexible morphing skin can have on the performance of the morphing wing.

Chapter 6 is a summary of all significant results presented in chapters 2 through 5. Main contributions of this research and future work will be presented in chapter 6 as well. 


\section{COMPUTATIONAL FLUID DYNAMICS AND NUMERICAL MODELING}

\subsection{Introduction:}

In the field of fluid dynamics, Computational Fluid Dynamics (CFD) is considered to be a new "third approach" in the philosophical study and development of this field. First, in the seventeenth century, the foundation of the experimental approach was established in England and France. The eighteenth and nineteenth centuries witnessed the formulations of many fluid dynamics theories that constitutes the second approach in studying fluid dynamics [31]. The advent of computational power and the level of accuracy reached by the numerical algorithms have promoted the usage of this third approach CFD when dealing with any new fluid dynamics problem. CFD can resolve the physical aspects of fluid flow to a level that cannot be reached by the experimental approach, and may require exhaustive efforts to be done analytically, if ever possible. For these reasons, and many others, the current research efforts mainly depend on the numerical results obtained from CFD and are backed up with experimental and analytical results when applicable.

The commercial CFD code ANSYS FLUENT V.15 is used in this project to numerically simulate the flow. This code uses the unstructured finite volume approach to discretize the computational domain and solve the governing continuity, momentum, energy and turbulence equations on a discretized domain. An implicit density-based solver is used with finite volume second order schemes to discretize the convection and diffusive fluxes of the transport equations. For proper resolution of the viscous boundary layer and its transition from laminar to turbulent, the turbulence of the flow was modeled using the four equation Langtry-Menter transitional shear stress transport turbulence model (Transition-SST model) [28 - 30]. Menter and Langtry [28] tested this model on several aerodynamic applications such as a flat plate, a Zierke and Deutsch compressor, an NREL wind turbine, an Aerospatiale A and the McDonald Douglas 30P-30N airfoils. Results showed that the wall shear stresses were well resolved and matched well the experimental data; an agreement that was not observed when a laminar model or fully turbulent models such as the k- $\varepsilon$ or the k- $\omega$ 
models were used. It should be noted here that one of the current limitations of the transition-SST model is that it does not account for crossflow instabilities and roughness [30].

Chapter 3 depends heavily on the Transition-SST model to predict the transition of the boundary layer from laminar to turbulent when traveling over cavities. It is claimed that this turbulence model is calibrated for an attached flow, and cannot be used with shear flows, as it is the case when flow travels over cavity. This claim can be disputed by the clear statement of Langtry and Menter in [30] when they mentioned that 'The present transition model accounts for transition due to freestream turbulence intensity, pressure gradients and separation', and flow separation is the reason of the transition in case of flows over cavities.

Among the validation test cases that the authors ran in [30] is the flow over the Pratt and Whitney PAK-B low pressure turbine blade. This profile encounters a laminar separation bubble on the suction side of the airfoil followed by a subsequent reattachment due to the transition of the boundary layer over the laminar separation bubble. The transition of the boundary layer occurs at the free shear layer due to the inflected velocity profile of the laminar separation bubble. This is the exact same transition mechanism that is experienced in case of flows over rectangular cavities. Even the main mode of instability in both cases is the same, namely, the Kelvin-Helmholtz instability. The RANS transition SST model managed to reach a very good agreement between the experimental and numerical data as shown in the figure (2.1). 


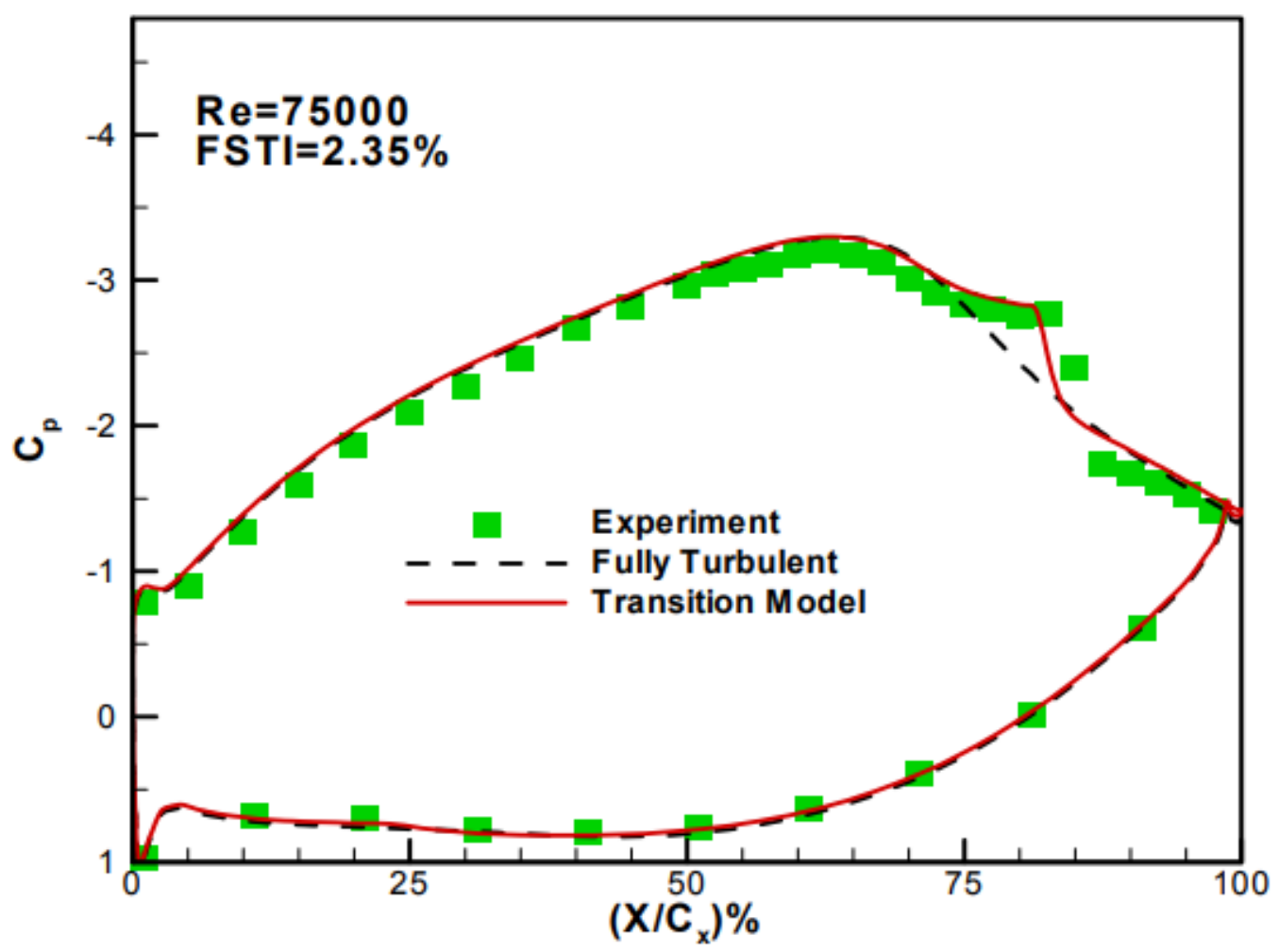

Figure 2.1. Predicted blade loading for the Pak-B Low-Pressure turbine at a Reynolds number of 75,000 and a freestream turbulent intensity of $2.35 \%$. Reprinted from [30].

Another example presented in [30] is the flow over the S809 airfoil. This airfoil is a $21 \%$ thick, laminar-flow airfoil that experiences laminar separation bubbles on both sides of the airfoil, and subsequent transition of the boundary layer at the bubbles locations ( $\sim 0.5$ of the chord length). The transition SST model precisely captured the locations of the bubbles, the shear layer transition, and reattachment of the flow. For all these reasons, the transition SST model was chosen to numerically model the turbulence of the flow and its transition when traveling over the airfoil and cavities. The author still has to mention here that the turbulence modeling in general, and the transition SST turbulence model is a major limitation in CFD.

Another critical procedure that must be followed in any CFD simulation practice is the choice of the optimal density of the grid. The grid must be fine enough to capture all the important flow features, but at the same time, it has to be fine enough to minimize the spatial discretization error of the simulation and capture all the rich physical features of the flow. To do that, the procedure proposed by Timothy Baker [32] is being followed for every case presented in this thesis. This approach provides an estimation of the order of convergence $p$ of the solution by tracking an aerodynamic property $\mathcal{F}$ on a family of three or more consecutively refined meshes. An aerodynamic property $\mathcal{F}$ is evaluated on the coarse, medium and fine meshes to obtain $\mathcal{F}_{c}, \mathcal{F}_{m}$ and 
$\mathcal{F}_{f}$ respectively. These values are extrapolated using Richardson's extrapolation method to calculate the continuum value $\mathcal{F}_{h=0}$ which represents the expected numerical value when the spacing between the nodes of the mesh tends to zero. The continuum value $\mathcal{F}_{h=0}$ is calculated from the three values $\mathcal{F}_{c}, \mathcal{F}_{m}$ and $\mathcal{F}_{f}$ as:

$$
\mathcal{F}_{\mathrm{h}=0} \cong \mathcal{F}_{\mathrm{f}}+\frac{\mathcal{F}_{\mathrm{f}}-\mathcal{F}_{\mathrm{m}}}{\mathrm{r}^{\mathrm{p}}-1}
$$

where $r$ is the refinement ratio from one mesh level to the other, and in our case it is constant and equals to 2. $p$ is the observed order of accuracy of the solution and is calculated as:

$$
p=\frac{\ln \left(\frac{\mathcal{F}_{\mathrm{c}}-\mathcal{F}_{\mathrm{m}}}{\mathcal{F}_{\mathrm{m}}-\mathcal{F}_{\mathrm{f}}}\right)}{\ln (\mathrm{r})}
$$

This order of accuracy can be also calculated from the logarithmic slope of the errors of the three meshes $\varepsilon_{c}, \varepsilon_{m}$ and $\varepsilon_{f}$. In this case, the error of $\mathcal{F}$ in each mesh is calculated as:

$$
\varepsilon_{\mathrm{c}}=\left|\mathcal{F}_{\mathrm{h}=0}-\mathcal{F}_{\mathrm{c}}\right|, \quad \varepsilon_{\mathrm{m}}=\left|\mathcal{F}_{\mathrm{h}=0}-\mathcal{F}_{\mathrm{m}}\right| \text { and } \quad \varepsilon_{\mathrm{f}}=\left|\mathcal{F}_{\mathrm{h}=0}-\mathcal{F}_{\mathrm{f}}\right|
$$

Even when using CFD algorithms with theoretical $2^{\text {nd }}$ order discretization schemes, the boundary conditions, the numerical models and the grid quality will reduce this order so that the observed order of convergence will likely be lower than 2 .

Through out the thesis there are several test cases that are 2D in nature, and other that are 3D. Some cases were solved using RANS and other were solved using URANS. The computational time of each case to reach convergence varied greatly. Table (2.1) lists the approximate wall clock time consumed by each case.

Table 2.1. Approximate wall clock time of different computational cases.

\begin{tabular}{cccc}
\hline Case & \# Cells & \# Cores & Wall Clock Time \\
\hline 2D NACA 2412 - Steady & 210,000 & 16 & $\sim 3$ hours \\
\hline 2D NACA 2412 - Transient & 210,000 & 16 & $\sim 8$ hours \\
\hline 3D NACA 2412 - Steady & $15,000,000$ & 16 & $\sim 3$ days \\
\hline
\end{tabular}


Another important decision that each CFD researcher has to make is the method used to reconstruct the gradient of scalar quantities over the control volumes. Despite years of research and developments in CFD codes and algorithms, very little is published on the effect of the gradient reconstruction method on the unstructured flow solvers. Even in literature, very little can be found about the compatibility of the gradient reconstruction method with the type of the mesh used. For that reason, an extra effort was done to thoroughly study three commonly used gradient methods, namely, the Green-Gauss cell based (GGCB) method, the Green-Gauss node based (GGNB) method, and the Least Squares cell based (LSCB) method. The study investigates the effect of the aforementioned gradient reconstruction methods on the flow solver when used with different grid types. The reader can refer to the following references [33-35] for more details.

The rest of this chapter will present a conclusion on which gradient reconstruction method is the most accurate in resolving the flow over morphing wings, taking into consideration the type of the mesh used. First, the numerical formulation of the three methods is presented, followed by a derivation of their formal order of accuracy on a 2D quadrilateral mesh. For more irregular meshes, formal derivations are exhaustively challenging, if even possible, so a numerical tool will be used to estimate the observed order of accuracy of each method on a wide range of mesh types. The practical implications of these results are tested on a full Navier-Stokes equations solver, and a comparison between the efficiency and accuracy of each method is presented. 


\subsection{Verification of the Gradient Reconstruction Method}

\subsubsection{Numerical Formulation of the Gradient Reconstruction Methods:}

The Green-Gauss cell based (GGCB) and the Green-Gauss node based (GGNB) methods are based on the discretized divergence theorem (a.k.a. the Green-Gauss theorem). The discretized form of the divergence theorem is given by:

$$
\nabla \phi=\frac{1}{\forall} \sum_{f}^{N_{\text {faces }}} \phi_{f} \boldsymbol{A}_{\boldsymbol{f}}
$$

Equation (2.4) states that the gradient of a certain scalar quantity $\phi$, over a control volume $\forall$ is estimated as the sum of the surface fluxes. The surface fluxes are calculated as the product of the surface value $\phi_{f}$ and the surface vector $A_{f}$. Both the GGCB and the GGNB methods use equation (2.4) to reconstruct the gradient $\nabla \phi$ at the center of each cell, but the way in which the face value $\phi_{f}$ is defined makes the difference between both methods.

\section{The Green-Gauss Cell Based Method}

The simple Green-Gauss cell based (GGCB) method calculates the face value $\phi_{f}$ as the simple average of the center values of two neighboring cells, thus the name 'Simple'.

$$
\phi_{f}=\frac{\phi_{P}+\phi_{Q}}{2}
$$

Equation (2.5) shows the simple averaging process between two neighbouring cells ' $\mathrm{P}$ ' and ' $\mathrm{Q}$ ' having center values of $\phi_{P}$ and $\phi_{Q}$. This simple averaging assumes equal contribution from each cell regardless of their geometric properties (aspect ratios, skewness, curvature, ... etc). Where $\phi_{P}$ and $\phi_{Q}$ are the scalar values at the centers of the two cells sharing a common face.

\section{The Green-Gauss Node Based Method}

The Green-Gauss node based (GGNB) method approximates the face value of the $\phi_{f}$ of the cells as an average of all the nodes enclosing the cell face as:

$$
\phi_{f}=\frac{1}{N_{f v}} \sum_{i=1}^{N_{f v}} \phi_{N_{i}}
$$

Where $N_{f v}$ is the number of nodes defining the face, and $\phi_{N_{i}}$ is the nodal value at the $i^{\text {th }}$ node. The nodal value $\phi_{N}$ is calculated using a highly robust approach that was first introduced by 
Holmes and Connell [36] in 1989, and represented by Rauch et al. [37] in 1991. In this approach, the nodal values $\phi_{N}$ is calculated as the weighted average of all center values of cells in direct contact with this node. This is achieved through an exact linear solution of the surrounding cells, thus the Laplacian of this linear function is exactly equal to zero in the $\mathrm{x}$ - and $\mathrm{y}$-directions. The weight $w_{i}$ of each cell's contribution is calculated by optimizing a cost function tending to reach unity for each weight. This optimization results in:

$$
w_{i}=1+\lambda_{x}\left(x_{i}-x_{0}\right)+\lambda_{y}\left(y_{i}-y_{0}\right)
$$

where $\lambda_{x}$ and $\lambda_{y}$ are the Lagrange multipliers. $\left(x_{0}, y_{0}\right)$ and $\left(x_{i}, y_{i}\right)$ are the components of the position vector of the node under consideration, and the center of the $i^{\text {th }}$ cell surrounding the node, respectively.

\section{The Least Squares Cell Based Method}

Unlike the GGCB and the GGNB methods which are based on the Green-Gauss theorem, the Least Squares cell based (LSCB) method approximates the gradient at the center of each cell using the least squares approximation. The gradient of each cell is assumed to change linearly along the separating distances from the center of the cell under consideration to the centers of all the neighbouring cells. In this case, the gradient could be written in a matrix form as:

$$
\left[\begin{array}{ccc}
\Delta r_{1 x} & \Delta r_{1 y} & \Delta r_{1 z} \\
\Delta r_{2 x} & \Delta r_{2 y} & \Delta r_{2 y} \\
\vdots & \vdots & \vdots \\
\Delta r_{N x} & \Delta r_{N y} & \Delta r_{N z}
\end{array}\right]_{N \times 3}\left[\begin{array}{c}
\nabla \phi_{0 x} \\
\nabla \phi_{0 y} \\
\nabla \phi_{0 z}
\end{array}\right]_{3 \times 1}=\left[\begin{array}{c}
\phi_{1}-\phi_{0} \\
\phi_{2}-\phi_{0} \\
\vdots \\
\phi_{N}-\phi_{0}
\end{array}\right]_{N \times 1}
$$

Equation (2.8) represents an over determinant system of equations, with a singular $N \times 3$ coefficient matrix on the left-hand side. The coefficient matrix is decomposed using the GramSchmidt process yielding a matrix of weights. Each of the neighbouring cells will have three weighting factors $w_{i}^{x}, w_{i}^{y}$ and $w_{i}^{z}$. The gradients in the $\mathrm{x}$ and $\mathrm{y}$ directions are calculated using:

$$
\nabla \phi_{0 x}=\sum_{i=1}^{N} w_{i}^{x}\left(\phi_{i}-\phi_{0}\right)
$$

and

$$
\nabla \phi_{0 y}=\sum_{i=1}^{N} w_{i}^{y}\left(\phi_{i}-\phi_{0}\right)
$$

The LSCB method ensures a monotonic solution over the computational domain, with an exact linear solution at the center of each cell. 


\subsubsection{Formal Order of Accuracy}

In this subsection, the formal order of accuracy of the three aforementioned gradient reconstruction methods are derived using a 2D quadrilateral mesh. This analysis will give an overview of the gradient operator's sensitivity to the geometric properties of the cells. A Taylor series is expanded around the neighbouring quadrilateral cells, with its center of expansion at $(i, j)$. Figure (2.2) is used to introduce the cells' terminologies used in the derivations.

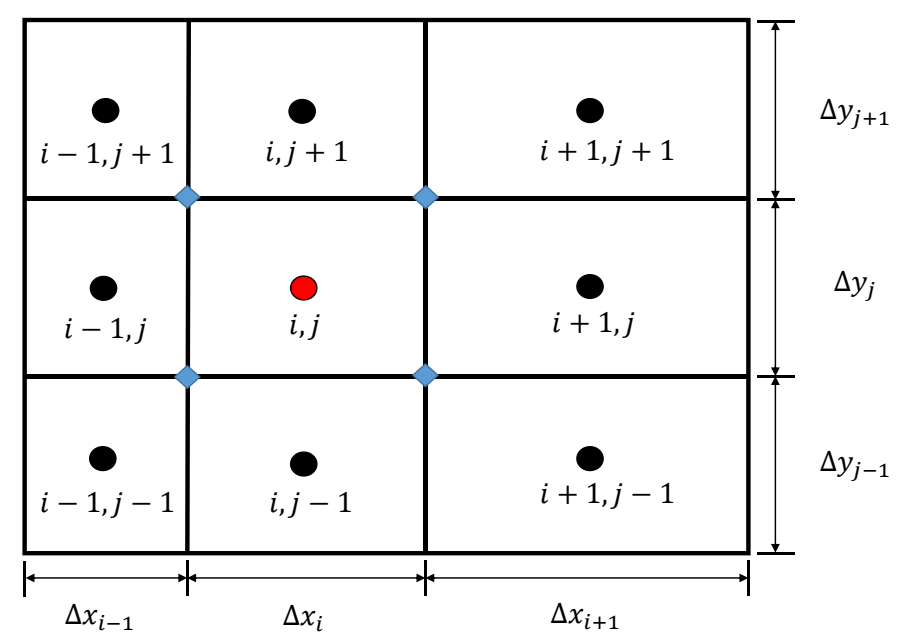

Figure 2.2. A quadrilateral cell at $\boldsymbol{i}^{\text {th }}$ and $\boldsymbol{j}^{\text {th }}$ position with all its neighbouring cells and mesh spacing.

This portion of the computational domain represents a number of quadrilateral cells with a constant growth rate $R$ along the $\mathrm{X}$ direction. Thus $\Delta x_{i+1} / \Delta x_{i}=\Delta x_{i} / \Delta x_{i-1}=R$. This is usually the case at the boundary layer of high Reynolds number simulations. The focus of the coming analysis will be on the $\mathrm{x}$-component of the gradient, thus the mesh is assumed to be equally spaced along the $\mathrm{y}$ axis.

For the GGCB method, the gradient in the x-direction of the cell $(i, j)$ is the summation of the surface fluxes at the surfaces $i+\frac{1}{2}$ and $i-\frac{1}{2}$, divided by the volume of the cell.

$$
\nabla \phi=\frac{1}{\Delta x_{i} \Delta y_{i}}\left[\phi_{i+\frac{1}{2}}-\phi_{i-\frac{1}{2}}\right] \Delta y_{i}
$$


Where the face values $\phi_{i+\frac{1}{2}}$ and $\phi_{i-\frac{1}{2}}$ are calculated as a simple averaging of the neighbouring cells as shown in:

$$
\begin{aligned}
& \phi_{i+\frac{1}{2}}=\frac{\phi_{i}+\phi_{i+1}}{2} \\
& \phi_{i-\frac{1}{2}}=\frac{\phi_{i}-\phi_{i+1}}{2}
\end{aligned}
$$

The center values of the neighbouring cells $\varphi_{i+1}$ and $\varphi_{i-1}$ can be approximated using a Taylor series expansion about the cell at $(i, j)$ as:

$$
\begin{aligned}
& \phi_{i+1}=\phi_{i}+\nabla \phi_{i} \frac{\Delta x_{i}+\Delta x_{i+1}}{2}+\nabla^{2} \phi_{i} \frac{\left(\Delta x_{i}+\Delta x_{i+1}\right)^{2}}{8}+O\left(\Delta x^{3}\right) \\
& \phi_{i-1}=\phi_{i}-\nabla \phi_{i} \frac{\Delta x_{i}+\Delta x_{i-1}}{2}+\nabla^{2} \phi_{i} \frac{\left(\Delta x_{i}+\Delta x_{i-1}\right)^{2}}{8}+O\left(\Delta x^{3}\right)
\end{aligned}
$$

By substituting equations (2.12 a) and (2.12 b) into equations (2.11 a) and (2.11 b), then back in equation (2.10), the $\mathrm{x}$ - component of the gradient at the center of the cell in figure (2.2) is given as:

$$
\begin{aligned}
& \nabla \phi_{x}=\nabla \phi_{i}+\nabla \phi_{i}\left(-\frac{1}{2}+\frac{\Delta x_{i+1}+\Delta x_{i-1}}{4 \Delta x_{i}}\right)+\nabla^{2} \phi_{i}\left(\frac{\Delta x_{i+1}-\Delta x_{i-1}}{8}+\frac{\Delta x_{i+1}^{2}-\Delta x_{i-1}^{2}}{16 \Delta x_{i}}\right)+ \\
& O\left(\Delta x^{2}\right)
\end{aligned}
$$

It could be directly deduced that the GGCB method will yield a $0^{\text {th }}$ order gradient on arbitrary mesh spacing. This is because the leading error in equation (2.13) directly contributes to the exact solution, and further refinements of the mesh will not diminish this error term. This means that the GGCB method is intrinsically inconsistent and its error is mesh dependent. Only a uniformly spaced mesh $\left(\Delta x_{i-1}=\Delta x_{i}=\Delta x_{i+1}\right)$ will nullify the second and third terms on the right-hand side of equation (2.13), and a $2^{\text {nd }}$ order accuracy is attained. Additional calculations showed that this observation applies on triangular elements as well. The same observation and interpretation of the $0^{\text {th }}$ order accuracy was documented by Sozer et al. [38].

A similar, but sizable procedure was followed to derive the formal order of accuracy of the GGNB method. The final formula is: 


$$
\nabla \phi_{x}=\nabla \phi_{i}+\nabla^{2} \phi_{i}\left(\frac{\Delta x_{i+1}-\Delta x_{i-1}}{8}\right)+O\left(\Delta x^{2}\right)
$$

Similarly, the formal order of accuracy of the LSCB method was derived as:

$$
\nabla \phi_{x}=\nabla \phi_{i}+\nabla^{2} \phi_{i} \times\left(\Delta x_{i+1}-\Delta x_{i-1}\right) \times \beta+O\left(\Delta x^{2}\right)
$$

Where $\beta$ is a combination of coefficient and given by:

$$
\begin{aligned}
& \beta=\left(\Delta x_{i-1}{ }^{2}+3 \Delta x_{i}{ }^{2}+3 \Delta x_{i} \Delta x_{i+1}+\Delta x_{i+1}{ }^{2}+\Delta x_{i-1}\left(3 \Delta x_{i}+\Delta x_{i+1}\right)\right) / \\
& 4\left(\Delta x_{i-1}{ }^{2}+2 \Delta x_{i-1} \Delta x_{i}+2 \Delta x_{i}{ }^{2}+2 \Delta x_{i} \Delta x_{i+1}+\Delta x_{i+1}{ }^{2}\right)
\end{aligned}
$$

Equations (2.12) and (2.13) show that the GGNB and the LSCB methods will achieve at least a $1^{\text {st }}$ order accurate solution on any type of meshes because of the $1^{\text {st }}$ order error terms. While on uniformly spaced meshes, the $1^{\text {st }}$ order errors are perfectly cancelled, and only $2^{\text {nd }}$ order errors are maintained. For that reason, the GGNB and the LSCB methods are considered to be linear exact gradient methods that will calculate the exact gradient of a linear function on any type of meshes. It should be noted here that for a $2^{\text {nd }}$ order convergence of the convection discretization error, at least a $1^{\text {st }}$ order gradient should be used $[38,39]$. This implies that the GGCB method can jeopardize the numerical solution in cases of non-uniform meshes. 


\subsubsection{Observed Order of accuracy}

As it has been shown in the previous section that deriving the formal order of accuracy of gradient reconstruction methods is an exhaustive task, even for regularly spaced 2D quadrilateral grids. So an alternative procedure will be shown in this section to estimate the observed order of accuracy of the gradient reconstruction methods using a numerical tool. A very effective and accurate method that showed to be successful in previous studies [38 - 42] is the isotropic scaling down method. When calculating the gradient at the center of any cell, all the nodes, cells' centers, and faces that form the stencil of the gradient of this cell are scaled down around a focal point located at the center of this cell. All the distances are scaled down by multiplying all distances of this cell's stencil by $1 / 2^{n}$, where $n$ represents the level of refinement. For $n=0$, the original mesh is retrieved, for $n=1$, all the distances are halved, while for $n=20$, all distances in the mesh are scaled down by $2^{20}=1,048,576$ times.

As the mesh is refined, the spatial discretization error will asymptotically reach zero, and the numerical solution will approach the continuum exact solution, and the logarithmic slope of the error will represent the order of accuracy of this gradient operator. Figure (2.3) shows an example of a cell isotropically scaled down by 2 refinement levels. The red dot represents the center of the cell at which the gradient is calculated, as well as the scaling down focal point. When $n=0$, the original grid is shown in black, when $\mathrm{n}=1$, all distances inside this grid are halved and shown by the blue lines. For $n=2$, all distances inside this grid are divided by 4 and shown by green lines.

Conventionally, structured grids are refined by introducing a midpoint node between each two existing nodes, then connectivity between newly introduced nodes is established. This global refinement can be consistent for structured grids, however, for unstructured meshes, the process is much more complicated. It will result in non-uniform refinement on the local scale of each cell, and globally in terms of the number of cells. The isotropic scaling method mitigates these problems by reproducing consistent refinements on all types of meshes, which is unattainable using conventional refinement methods. In addition, this method can produce extensively refined meshes using a single generated mesh. The most important feature of this method is that the computational time is not altered from one refinement level to the other. This makes the isotropic scaling down method a very attractive and efficient testing tool.

The order of accuracy of each method is evaluated using the method of manufactured solution. This means that the exact solution at each cell is known. The error of the reconstructed gradient at the $i^{\text {th }}$ cell and the $n^{\text {th }}$ refinement level is calculated as:

$$
\epsilon_{i}^{n}=\left|\nabla \varphi_{i, \text { numerically }}^{n}-\nabla \varphi_{i, \text { exact }}\right|=C h^{O O A_{i}}+H . O . T .
$$




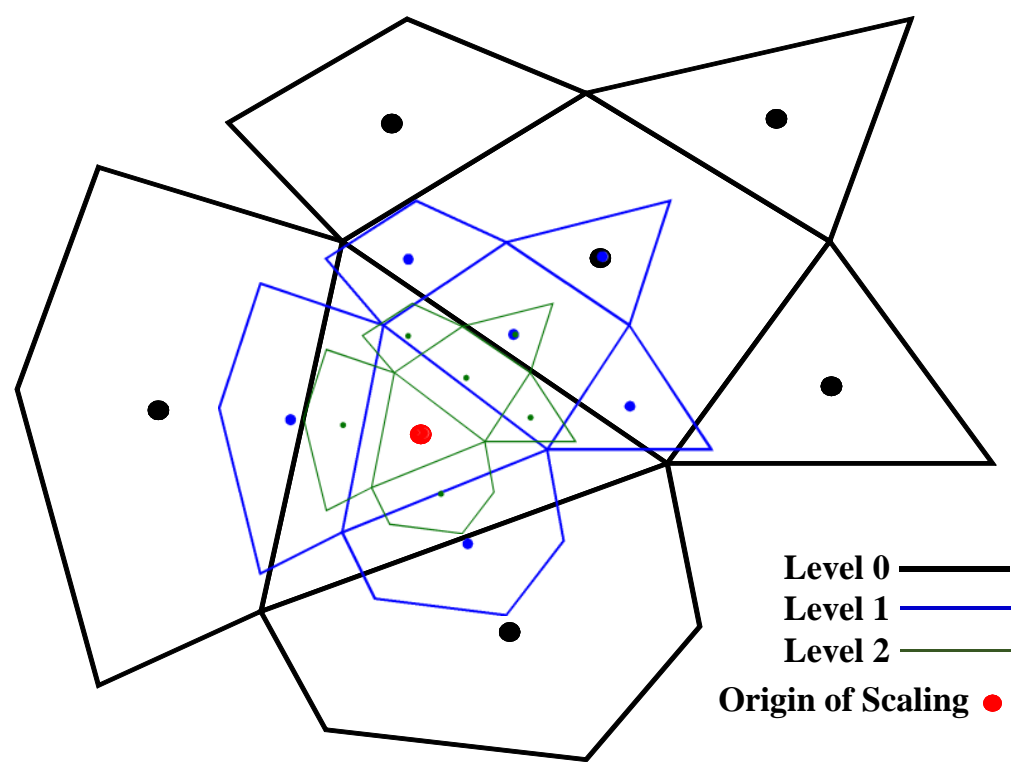

Figure 2.3. The isotropic scaling down method with the origin of scaling at the cell's center.

Where $C$ is a constant, $h$ is a measure of the mesh spacing, and $O O A_{i}$ is the order of accuracy at the $i^{\text {th }}$ cell. By neglecting the higher order terms (H.O.T.), and taking the logarithm of both sides, the result is:

$$
\log \left(\epsilon_{i}^{n}\right)=\log (C)+O O A_{i} \log (h)
$$

The order of accuracy of the solution at the $i^{t h}$ cell is then calculated as the logarithmic slope of the errors between two successive refinement levels as:

$$
O O A_{i}=\frac{\log \left(\epsilon_{i}^{n} / \epsilon_{i}^{n+1}\right)}{\log (2)}
$$

The denominator in equation (2.17) is $\log (2)$ because the mesh is scaled down by a factor of 2 from one refinement level to the other $\left(h_{i}^{n} / h_{i}^{n+1}=2\right)$.

To conveniently test a wide range of mesh types, several types of meshes are assembled on a single computational domain. The mesh consists of quadrilateral elements, right angled triangular elements and equilateral triangles that range from equally spaced to totally perturbed elements. Figure (2.4) shows the computational domain used to test the gradient operators. 

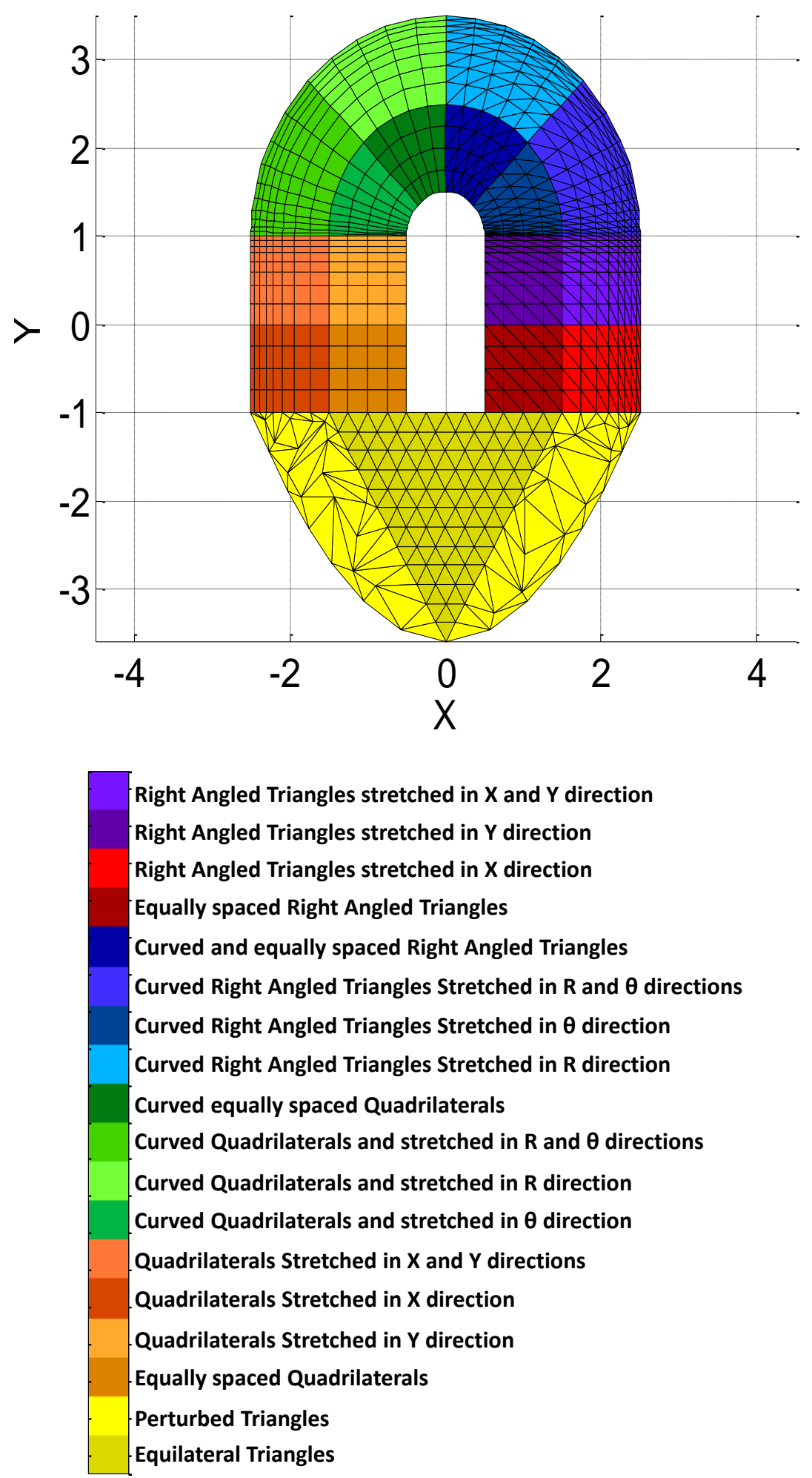

Figure 2.4. Different types of meshes that will be used to assess the order of accuracy of the three gradient operators. 
The hybrid computational domain in figure (2.4) consists of commonly used mesh elements in CFD applications. Quadrilaterals and curved quadrilaterals with their stretched variations are widely used to resolve the viscous boundary layers at high Reynolds numbers. The right angled triangular elements are also used at the viscous boundary layers to increase the CFD solver accuracy and convergence rate [43]. While equilateral triangles and their perturbed version usually represents the core elements of the computational domain in unstructured grids.

The testing function used was Ackley's function shown in figure (2.5). This function:

$$
f(x, y)=-20 \exp \left(-0.2 \sqrt{0.5\left(x^{2}+y^{2}\right)}\right)-\exp (0.5(\cos (2 \pi x)+\cos (2 \pi y)))+e+20
$$

is a benchmark equation widely used to test optimization and numerical algorithms in applied mathematics. It consists of a large funneled shape structure with its absolute minimum at the origin and the surface of the funnel has a rough texture with many local minima.

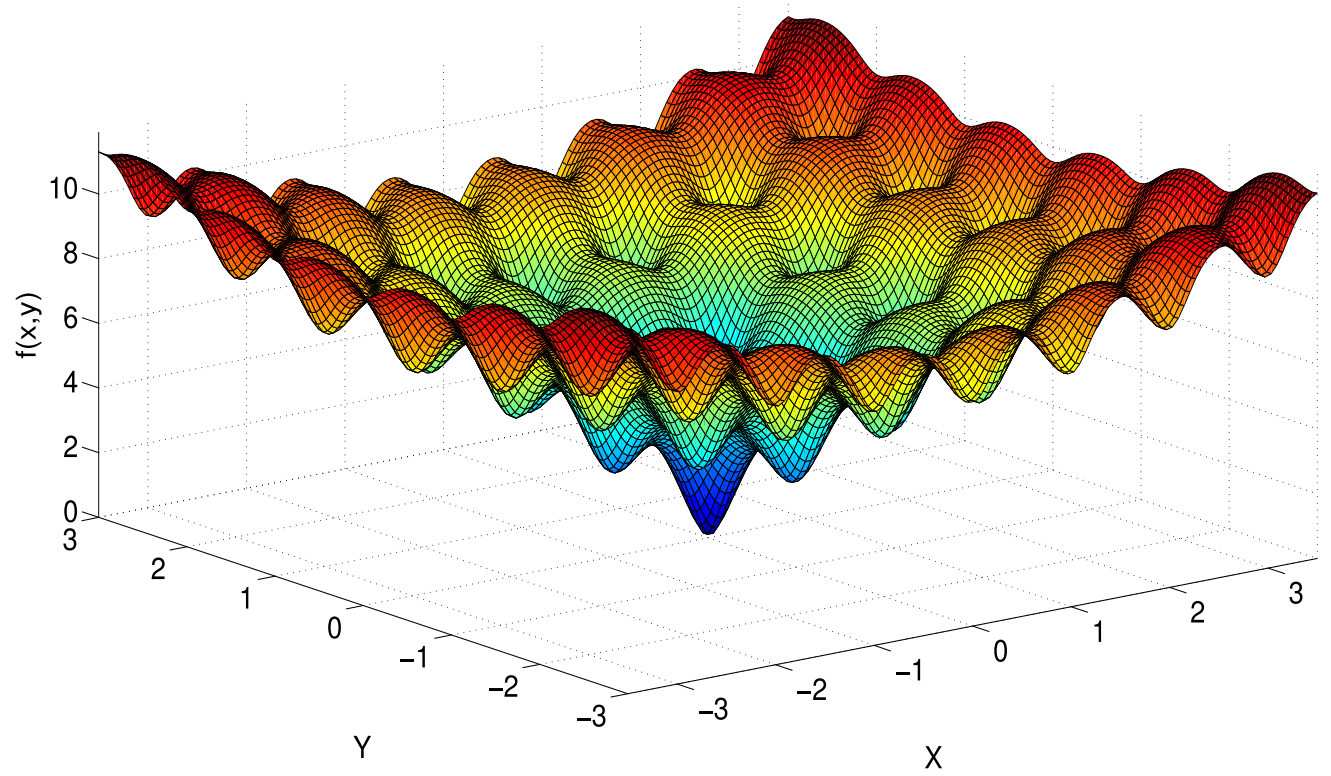

Figure 2.5. 3D representation of Ackley's testing function.

The gradient of the testing function was calculated at each cell in the computational domain using the three gradient reconstruction methods. The mesh was refined for 20 refinement levels, and at each level the gradient was calculated. Figure (2.6) shows an example of the $\mathrm{L}_{1}$ norm convergence of the $\mathrm{x}$ and $\mathrm{y}$ gradients on four variations of the curved quadrilateral elements colored by shades of green in figure (2.4). 


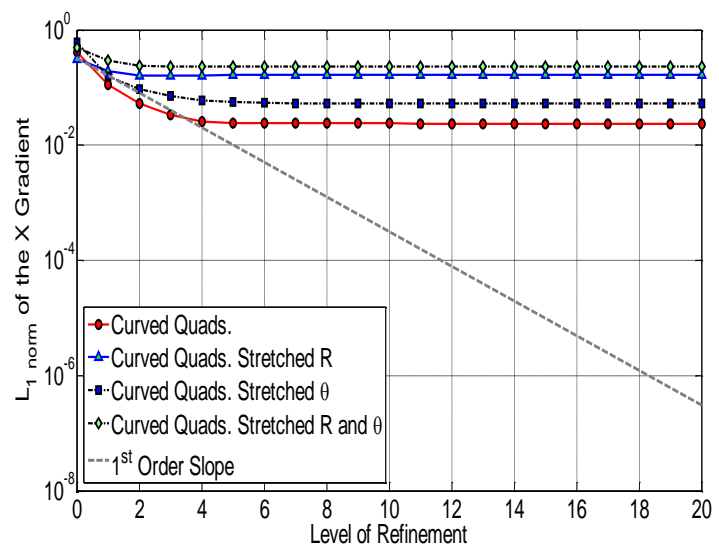

(a) X gradient of the GGCB method

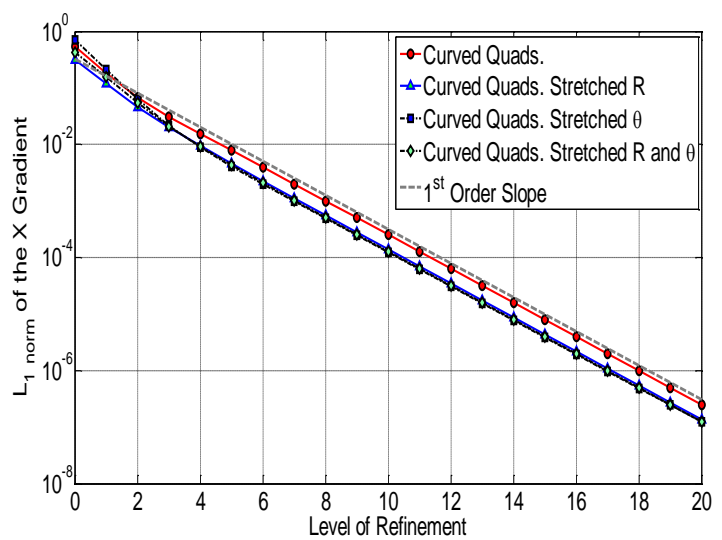

(c) X gradient of the GGNB method

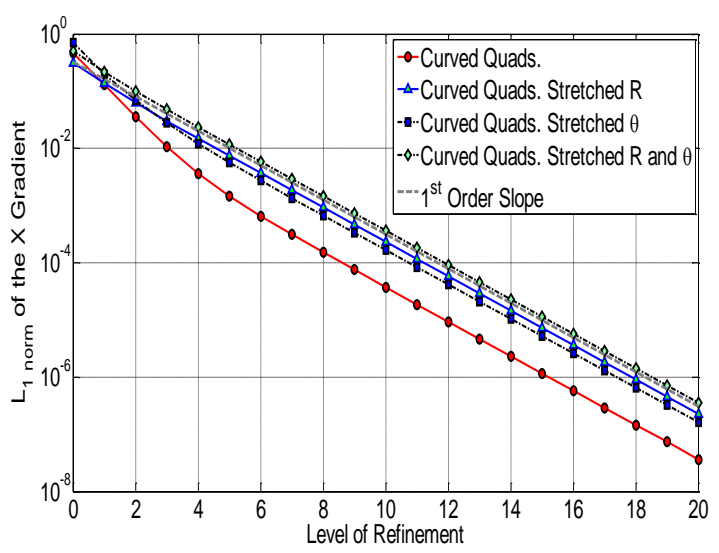

(e) X gradient of the LSCB method

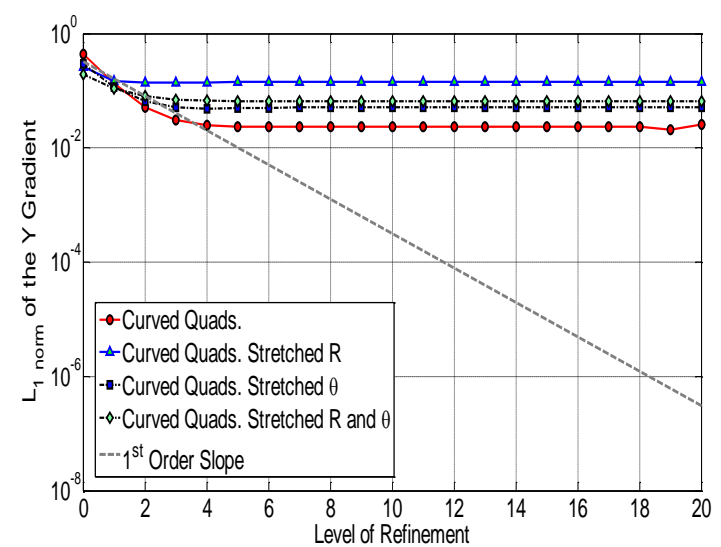

(b) Y gradient of the GGCB method

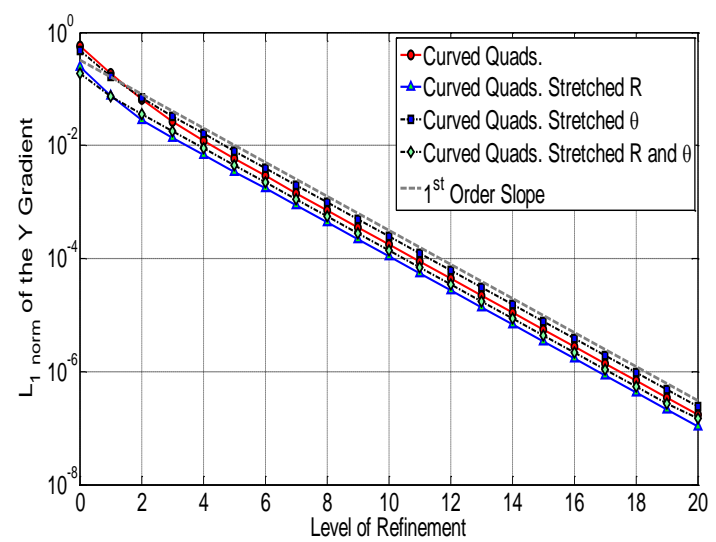

(d) Y gradient of the GGNB method

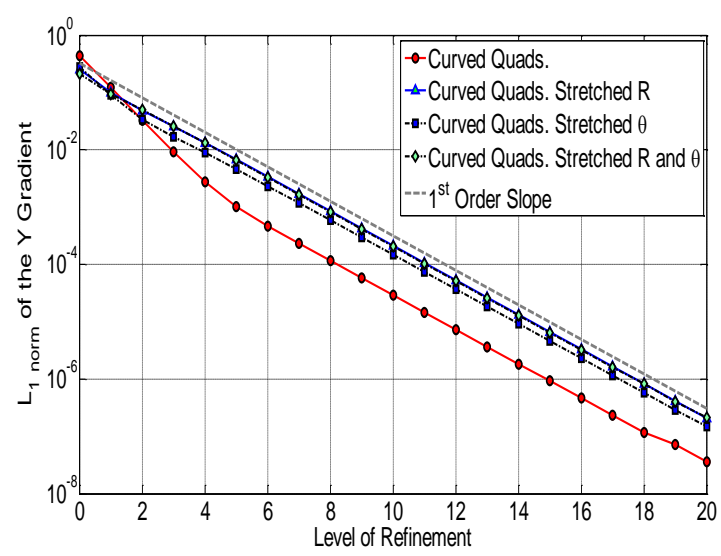

(f) Y gradient of the LSCB method

Figure 2.6. L1 norm convergence of solution on curved quadrilateral mesh using the three gradient reconstruction methods. 
The zero slopes of the lines in figures (2.6 a) and (2.6 b) show that the GGCB method achieved a $0^{\text {th }}$ order accuracy on the curved quadrilaterals. On the other hand, the first order slopes in figures $(2.6 \mathrm{c}-\mathrm{f})$ show that the GGNB and the LSCB methods obtained a $1^{\text {st }}$ order accuracy over all refinement levels. A more convenient and compact way of representing the results on all types of meshes is shown in figure (2.7). The results of the $12^{\text {th }}$ and $13^{\text {th }}$ refinement levels are used to calculate the order of accuracy of each cell using equation (2.17). Each element in the hybrid mesh is color coded with a color representing the achieved order of accuracy $\left(0^{\text {th }}, 1^{\text {st }}\right.$ or $2^{\text {nd }}$ order $)$.

Figures (2.7 a) and (2.7 b) show that the GGCB method achieved $2^{\text {nd }}$ order accurate solutions only on quadrilateral elements that are equally spaced along the direction of the gradient component.

This accuracy dropped to $1^{\text {st }}$ order on equally spaced triangular elements, and dropped again to a $0^{\text {th }}$ order accuracy when used on any other type of meshes. This is because the leading error of the gradient directly contributes to the exact solution, and further refinements of the mesh will not diminish this error term. This means that the GGCB method is intrinsically inconsistent and its error is mesh dependent. Only a uniformly spaced mesh will nullify the $1^{\text {st }}$ order error terms and a $2^{\text {nd }}$ order accuracy will be attained. In figures $(2.7 \mathrm{c}-\mathrm{f})$, the GGNB and the LSCB methods maintained a $2^{\text {nd }}$ order accurate solution on equally spaced quadrilaterals. This is due to the perfect cancellation of the $1^{\text {st }}$ order errors. While on all other types of meshes, the GGNB and the LSCB methods achieved a $1^{\text {st }}$ order accuracy. This means that they are capable of exactly reproducing the gradient of a linear function on any type of meshes.

Although the GGNB and the LSCB methods achieved similar orders of accuracy, the numerical formulation of the LSCB method is much simpler than the GGNB method, thus computationally less expensive as it will be shown in the next sections. 


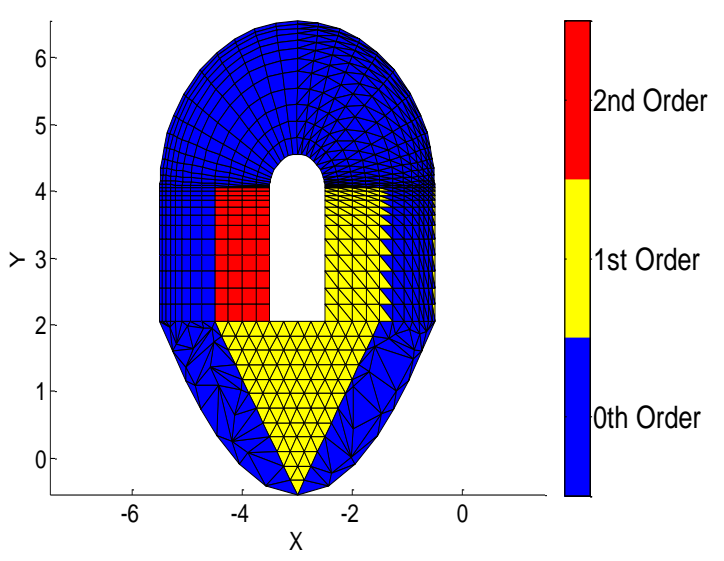

(a) X - gradient using the GGCB method

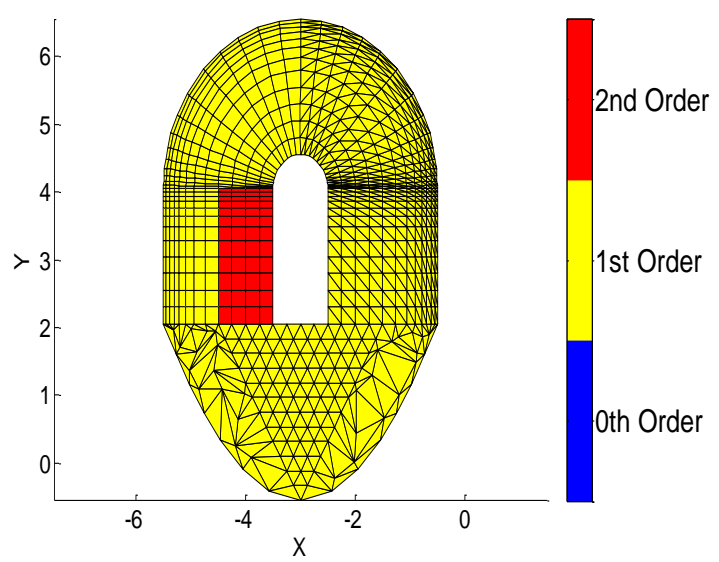

(c) $\mathrm{X}$ - gradient using GGNB method

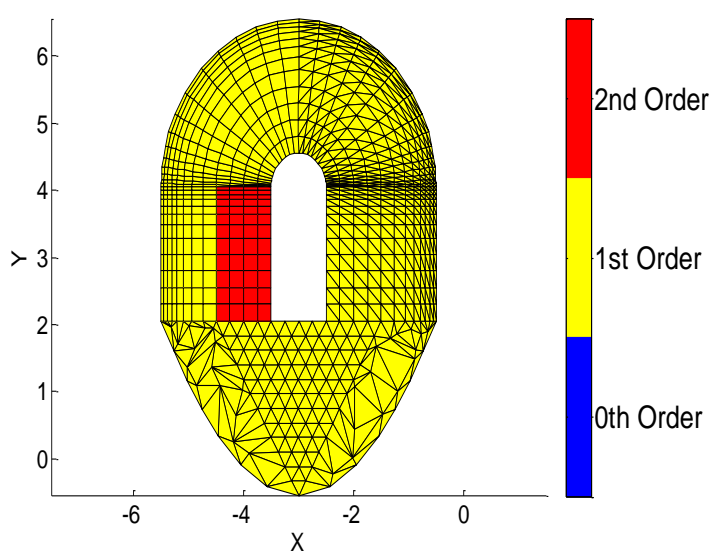

(e) X - gradient using LSCB method

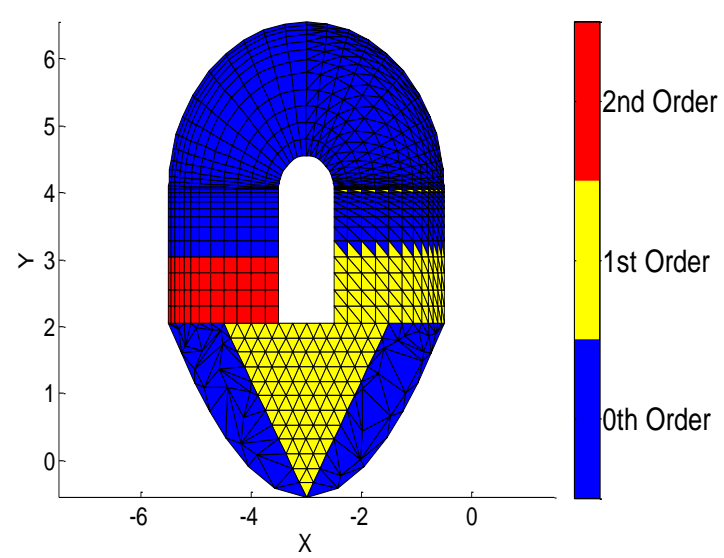

(b) Y - gradient using the GGCB method

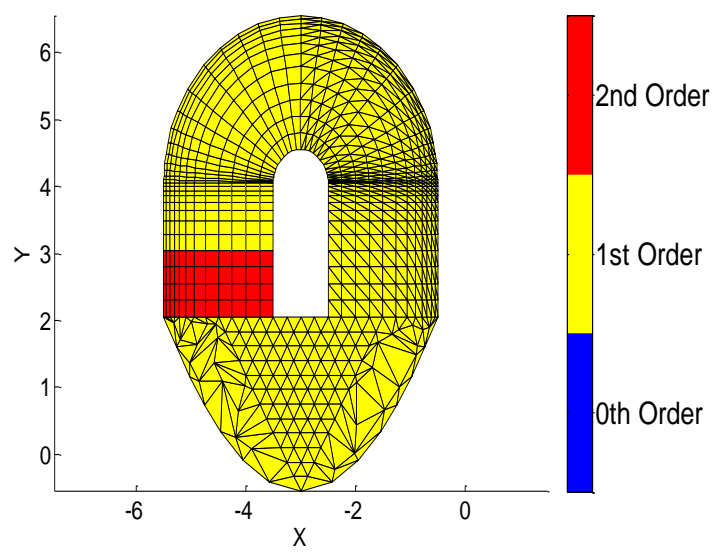

(d) Y - gradient using GGNB method

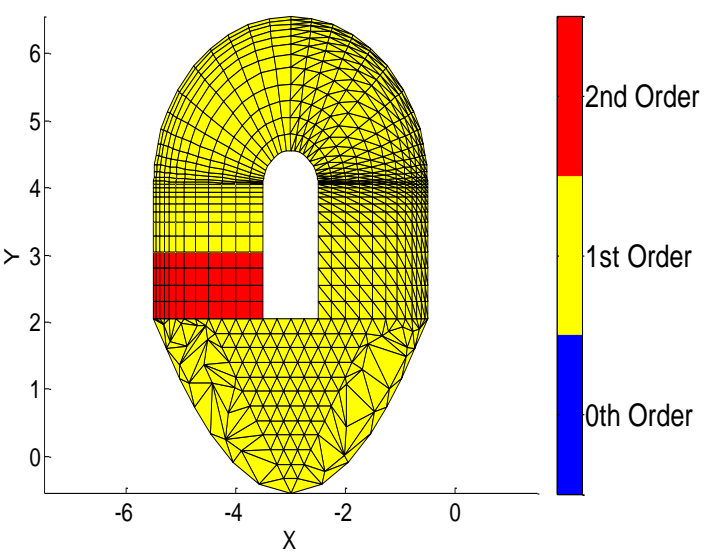

(f) Y - gradient using LSCB method

Figure 2.7. Observed order of accuracy of the reconstructed gradients, calculated using the three gradient reconstruction methods. 


\subsubsection{Practical Implications}

Spatial derivatives obtained by the gradient operators are used in several equations in CFD codes; starting from the diffusion and convection terms of the momentum equations, to the energy equation, to any additional transport equations. This makes tracing the effect of the gradient operators on the final solution an exhausting process. Despite the numerous studies that are conducted on CFD algorithms, the effect of the gradient reconstruction method on the final flow solution is still far from clear. In this section, a numerical methodology is adopted to investigate the effect of the three aforementioned gradient operators on the solution of high aspect ratio CFD solutions.

Four Aerodynamics applications will be tested in this study. In the first case, the Euler flow equations are solved on a NACA 0012 airfoil. The second case focuses on the viscous forces at the boundary shear layer of a flat plate. In the third and fourth cases, the flow between an outer stationary cylinder and an inner rotating one is induced to create a rotating Couette flow. The four cases represent four different types of meshes, and each is solved on a family of consecutively refined grids as shown in figure (2.8).

In each case, the flow is solved three times using the CFD commercial code FLUENT V15, each time a different gradient reconstruction method is used. The Richardson's extrapolation method is used to estimate the order of convergences of each gradient method when used with each case. While keeping in mind the importance of the numerical efficiency, the computational time consumed by the solver to reach convergence is used as a comparator measure for the efficiency of each gradient operator.

The computational time is calculated as the time consumed by a single Intel Xeon $2.6 \mathrm{GHz}$ processor to reach convergence. Convergence of the solution is considered to be reached when the value of $\mathcal{F}$ is changing at a rate less than $0.001 \%$ of its value from one iteration to the other. This criterion aims at comparing the efficiency of each gradient operator when used with the full set of flow equations. The description of the geometry, boundary conditions and flow features of each case are presented below. 


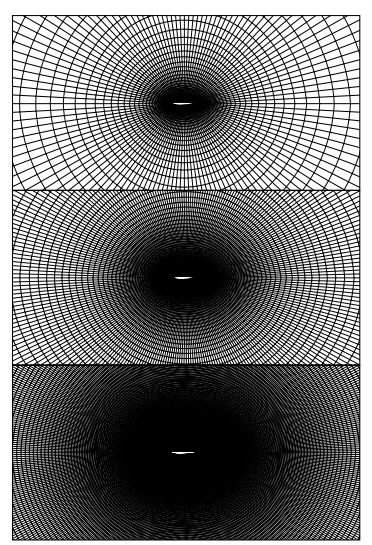

(a)

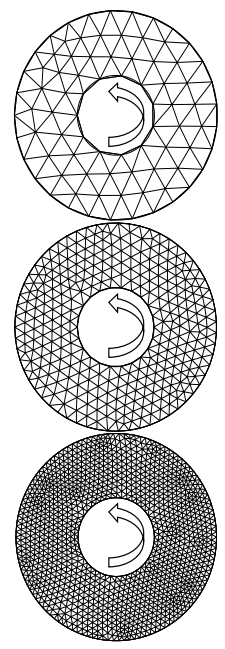

(c)

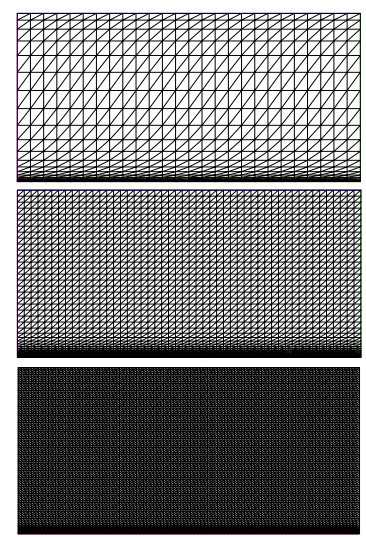

(b)

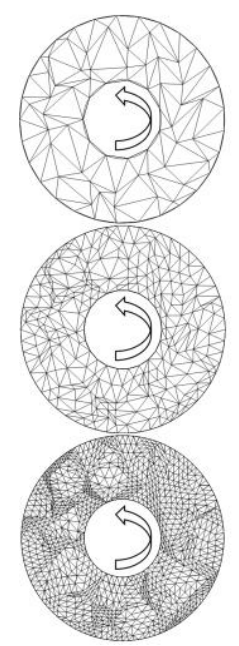

(d)

Figure 2.8. Three consecutively refined meshes used with (a) the NACA0012, (b) the flat plate, (c) rotating Couette flow on equilateral triangular mesh and (d) rotating Couette flow with perturbed mesh.

\section{Case 1: Euler solution over NACA0012 airfoil}

In this test case, the flow around a NACA 0012 airfoil is simulated using Euler's equations. The flow is assumed to be inviscid, and this assumption will significantly reduce the computational time by eliminating the refined mesh used to resolve the viscous boundary layer. The fast converging Euler equations increase the feasibility of using finer refinements for the grids, and thus a more accurate estimation of the solution's order of accuracy.

The airfoil used is a standard NACA 0012 airfoil with a sharp trailing edge. The chord length of the airfoil was set to $1 \mathrm{~m}$. An O-mesh topology was created around the airfoil with an extended far-field boundary that is 150 chords away from the airfoil. A family of consecutively refined grids 
was created with a constant refinement factor of 2 . The coarse mesh consisted of 64 nodes around the airfoil and extends to the far-field through 64 levels, thus the course mesh is $64 \mathrm{x} 128$ cells. The medium and fine meshes were created from the coarse mesh by constant uniform refinements of 2. Figure (2.8 a) shows the grids created for testing the NACA 0012 airfoil.

The flow around the airfoil was set to a subcritical flow condition with a Mach number of 0.5. An angle of attack of $\alpha=1.25^{\circ}$ was used to create a non-zero lifting force on the airfoil, and consequently a pressure drag. The drag coefficient $C_{d}$ was chosen to be the scalar property $\mathcal{F}$ for the Richardson's extrapolation analysis and will be used to estimate the numerical order of accuracy $p$.

\section{Case 2: Laminar flow over a flat plate}

With the focus on the first case was on the inviscid flow, the second case will focus on the viscous forces in the boundary layer of a flat plate. This restores the diffusion term back to the momentum equation. The geometry of the plate and the computational domain are shown in figure (2.9).

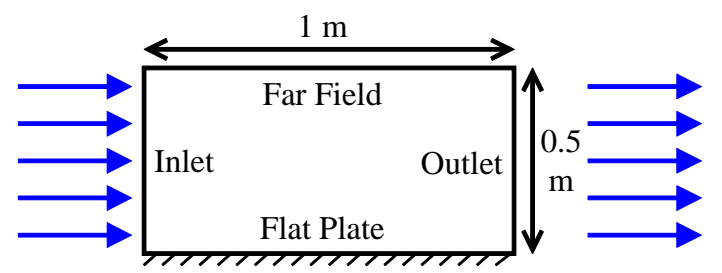

Figure 2.9. Geometry and computational domain of the flat plate test case.

The flat plate extends for 1 meter and the height of the computational domain is chosen to be approximately ten times the boundary layer thickness which could be approximated using Blasius

equation $\left(\delta_{99} \approx \frac{4.91 x}{\sqrt{R e_{x}}}\right)$. The three consecutively refined meshes that are used in this test case consist of right angled triangular elements as shown in figure $(2.8 \mathrm{~b})$. This type of mesh introduces a sort of computational complexity in reconstructing the gradient near the flat plate. This is due to the inclined surfaces of the triangular elements and the high aspect ratios near the boundary layer. Reynolds number due to length $R e_{x}$ of the flat plate is chosen to be 10,000 . The flat plate is treated as an adiabatic wall, and the upper far field of the computational domain is a symmetric axis, thus no refinement is needed at this boundary. The skin friction coefficient at $x=0.85 m\left(C_{f_{0 x=0.85}}\right)$ was chosen as the scalar property $\mathcal{F}$ for Richardson's extrapolation, and for estimating the numerical order of accuracy $p$.

\section{Cases 3 and 4: Rotating Couette flow}

In the third and fourth cases, the famous rotating Couette flow is solved numerically on an equilateral triangular and a perturbed triangular mesh respectively. This type of flows consists of 
a fluid that is entrapped between an inner rotating cylinder and an outer stationary drum. The rotation of the inner cylinder drags the next-to-the-wall layers along with it. This induces the flow between the cylinders as shown in figure (2.10).

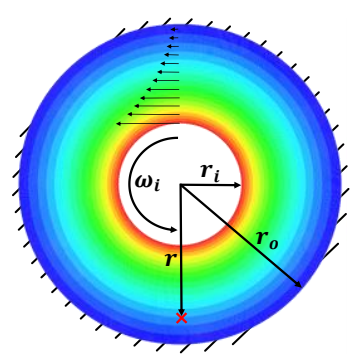

Figure 2.10. Geometry and computational domain of outer and inner cylinder of the rotating Couette flow.

In our case, the inner radius $r_{i}$ and the outer radius $r_{o}$ of the cylinders are set to $17.8 \mathrm{~mm}$ and $46.8 \mathrm{~mm}$ respectively. The inner cylinder is rotating at an angular velocity of $\omega_{i}=1 \mathrm{rad} / \mathrm{s}$. This rotation induces the flow between the cylinders whose viscosity is equal to $0.0002 \mathrm{~kg} / \mathrm{m} \mathrm{s}$. The value under consideration $\mathcal{F}$ is the tangential velocity at radius $r=35 \mathrm{~mm}$. 


\subsubsection{Results and Discussion}

Each of the four cases was solved on three different levels of refined meshes, each with the three aforementioned gradient operators. The first style that will be compared is the observed order of accuracy of the solution. Equations $(2.1-2.3)$ were used to estimate the error of the aerodynamic property $\mathcal{F}$ on each mesh of the four cases. The convergence of the spatial truncation error of $\mathcal{F}$ is plotted in figures $(2.11 \mathrm{a}-\mathrm{d})$.

The slopes of the straight lines in the figures represent the order of accuracy of the solution. Figure (2.11 a) shows the deviation of the calculated drag coefficient in each of the nine runs when compared to its corresponding continuum value $C_{d_{h=0}}$. Both the GGNB and the GGCB methods yielded solutions with slopes close to each other, with values of 1.6913 and 1.6537, respectively. While the LSCB method achieved a value of 1.8712 which is close to the theoretical value of 2 for $2^{\text {nd }}$ order discretization schemes.

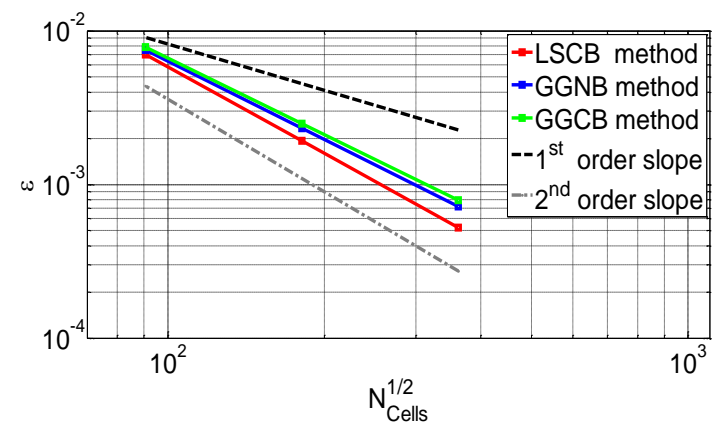

(a) Case 1

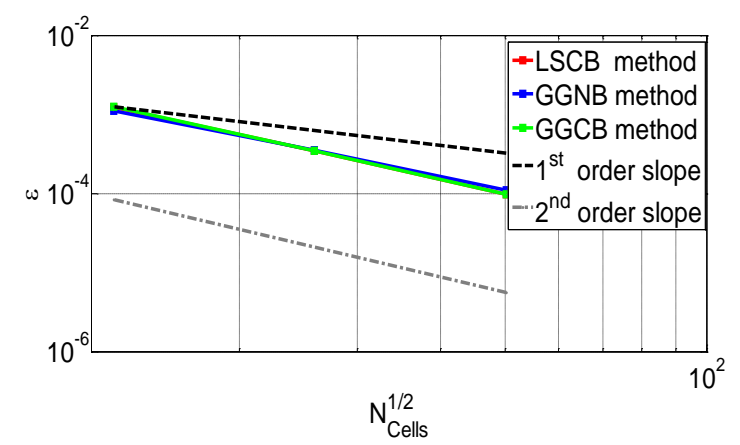

(c) Case 3

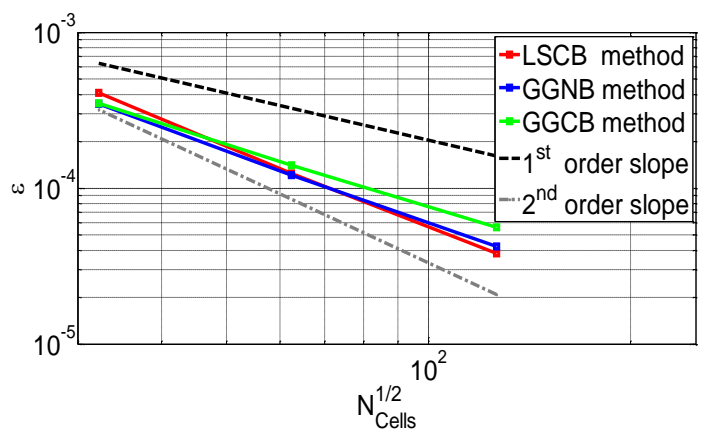

(b) Case 2

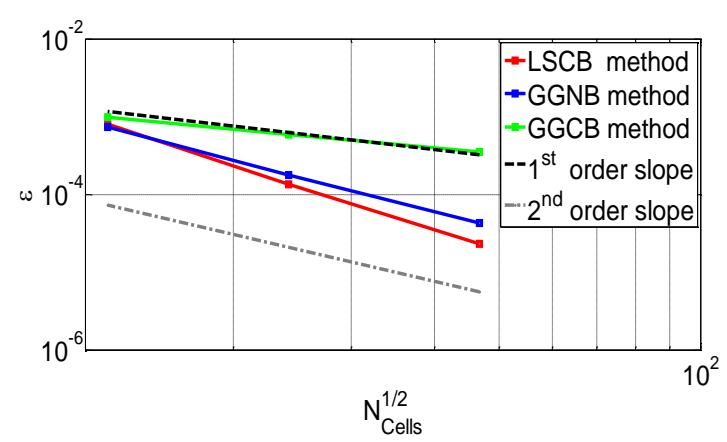

(d) Case 4

Figure 2.11. Convergence of the spatial truncation error in the four cases. 
In case 2, the GGCB method achieved the least accuracy with a slope of 1.3428, then the GGNB resulted in an observed order of accuracy of 1.5466, and the LSCB method achieved the highest accuracy of 1.7347 . These results are plotted in figure $(2.11 \mathrm{~b})$.

The equilateral triangular elements in case 3 resulted in orders of accuracies that are close to each other in cases of the three gradient operators. The calculated slopes of the lines in figure $(2.11 \mathrm{c})$ are found to be 1.88, 1.708 and 1.86 for the GGCB, the GGNB and the LSCB methods respectively. These results were expected from figure (2.7), where on meshes with equidistant spacing, the three gradient operators obtain the same $1^{\text {st }}$ order accuracy.

The perturbed meshes in case 4 revealed the disability of the GGCB method to handle the viscous and inviscid fluxes on highly skewed triangular (or quadrilateral) elements. The GGCB method fell below the $1^{\text {st }}$ order accuracy with a value of 0.80781 despite using $2^{\text {nd }}$ order discretization schemes with the flow solver. In the second place came the GGNB method and the LSCB method was the most accurate. The calculated order of accuracy of the former two methods slightly exceeded the $2^{\text {nd }}$ order accuracy. This is due to the inconsistent refinement of the perturbed triangular mesh that results in non-uniform mesh density over the computational domain.

The second test of the gradient reconstruction methods assessment compares the efficiency of each gradient operator. The computational running time consumed by the solver to reach convergence on the finest mesh is used as a measure to compare the gradient operator's efficiency.

The intent of this study is to compare the performance of each gradient operator when used on different types of meshes, and not to calculate the CPU time of the solution. For that reason, the measured computational time in each case is normalized by the minimum time required to reach convergence with each of the three operators. In the four cases, the GGCB method was the first to reach convergence, thus its non-dimensional time is equal to 1 in all cases as shown in figure (2.12).

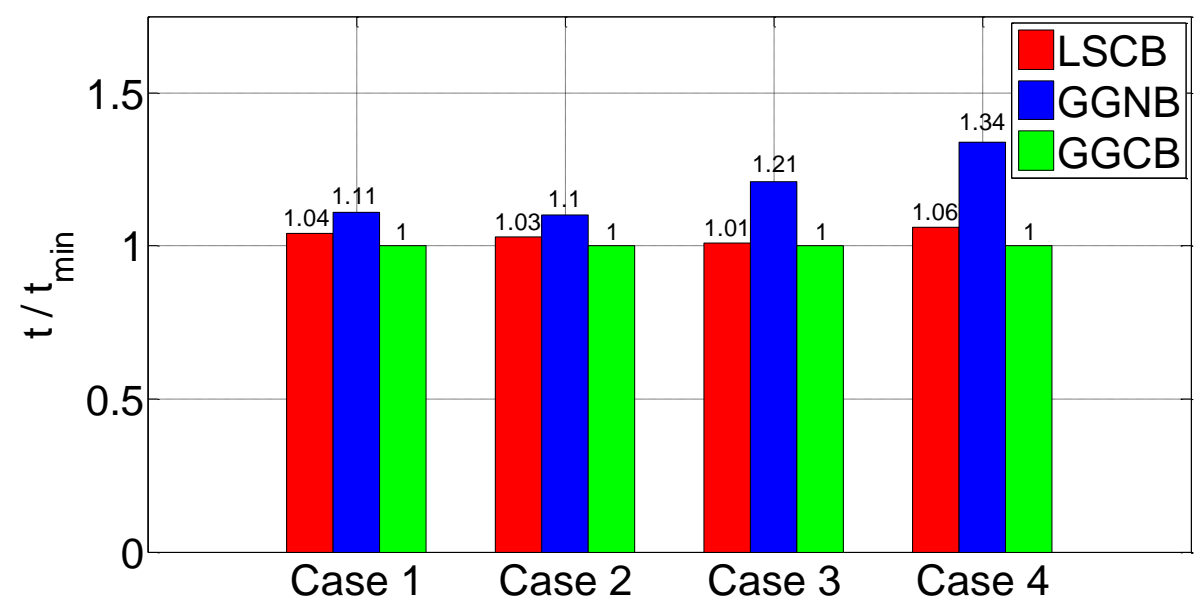

Figure 2.12. Comparison of the computational expenses of the gradient operators for the four cases. 
The GGCB method was always the first to reach convergence because of its simple formulation that calculates the face value $\phi_{f}$ as the average of the neighbouring cells. On the other hand, the GGNB method consumed about $9-34 \%$ additional time when compared to the GGCB. This is due to the complexity of the GGNB method that involves in the calculations the nodal value at each vertex of each cell. While the relative simplicity of the LSCB method resulted in an efficient algorithm with computational expenses that are well comparable to the GGCB method. The LSCB method consumed a maximum of $6 \%$ additional time when compared to the GGCB method.

These analyses have shown that the LSCB gradient reconstruction method offers a reasonable trade-off between accuracy and efficiency. For that reason, all the numerical test cases in the next chapters will use the LSCB gradient reconstruction method to resolve the flow over morphing wings and morphing skins. 


\subsection{Validation of the NACA 2412 CFD Results}

After reaching a conclusion that the least squares cell-based method is the most accurate and efficient method, the CFD solver options are now set. It is of a great convenience for the author to present here a validation test case that will give confidence to the readers in the CFD tool used in this entire thesis. Despite presenting quantitative and qualitative validation cases in chapter 3 for flows over cavities, a general validation case for flow over an airfoil is essential.

As mentioned earlier in section 1.2, the exact model of the aircraft was not provided by Bombardier Inc., but the averaged Reynolds number at the wing tip was calculated to be approximately 5.7 million. For that reason, most of the investigations in this thesis are done at a Reynolds number of 5.7 million, and a Mach number of 0.17 to match the experimental conditions reported by Abbott and Von Doenhoff [44]. This Reynolds number is in accordance with the average of Reynolds numbers of UAV's which is the prime customer of the morphing wing technology.

In this section, flow over a NACA 2412 will be simulated using CFD at difference angle of attacks, and the numerical results will be compared to the experimental values presented by Abbott and Von Doenhoff in [44]. The NACA 2412 is used as the baseline airfoil for flow simulations in this thesis because it is commonly used for airplanes designed to operate at both subsonic and supersonic speeds. This is in addition to their applications in helicopter rotor blades and highperformance propeller blades [44, 45].

Abbott and Von Doenhoff [44] tested the airfoil at three Reynolds numbers, namely 3.1, 5.7 and 8.9 million. The Mach number reported by them was not exact, but they mentioned that it is slightly lower than 0.17. The wind tunnel used has a test section that is 3 feet wide and 7 feet high. The wing model has a chord length of 2 feet and a span of 3 feet. The lift of the wing is measured by integrating the pressure reactions on the floor and ceiling of the wind tunnel. The drag on the airfoil is obtained using the wake survey method presented in [46].

The CFD tool was set for a Reynolds number of 5.7 million and a Mach number of 0.17 . The computational domain extents for 32 chords, and a C-topology mesh was used where the blocks are wrapped around the sharp trailing edge airfoil. A mesh independent study was carried-out on a family of three consecutively refined meshes as described in section 2.1. Richardson's extrapolation method showed that the finest mesh is the most adequate mesh to resolve the flow where the error between the extrapolated asymptotic value of the lift coefficient and the value of the finest mesh is $0.87 \%$ at an angle of attack of $8^{\circ}$. Since the turbulent intensity was not reported in [44], the transition-SST turbulence model was manually tuned to a turbulent intensity of $1.75 \%$ and a surface roughness of $5 \mathrm{e}-5 \mathrm{~m}$.

The angle of attack was swept over the range of $0^{\circ}$ to $20^{\circ}$, and in each case the lift coefficient and drag coefficient were calculated. Figure (2.13) shows a comparison between the results obtained 
experimentally by Abbott and Von Doenhoff [44], and the results obtained from the CFD simulations at a Reynolds number of 5.7 million. Figure ( $2.13 \mathrm{a})$ shows the obtained lift coefficient $\mathrm{Cl}$ at different angle of attacks, and figure $(2.13 \mathrm{~b})$ shows the obtained drag coefficient at different lift coefficient values.

As it can be observed from figure (2.13 a) that a very good agreement is achieved at high angles of attack, and a perfect agreement is observed over the linear range at low angles of attack.

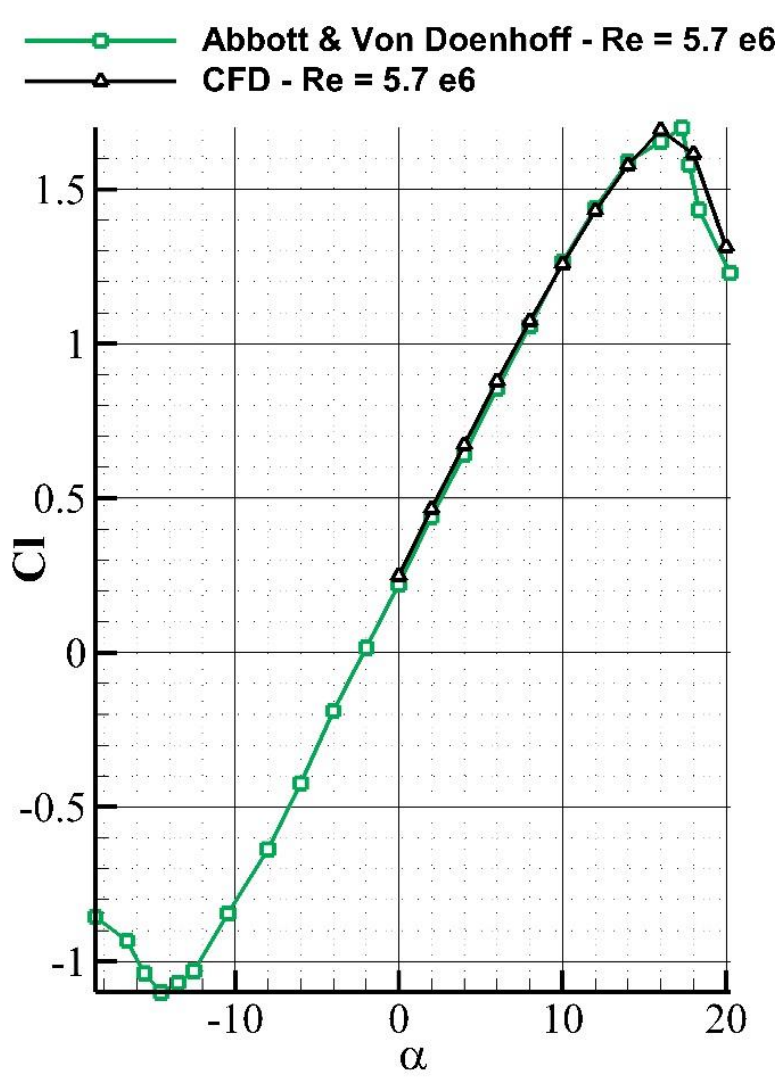

(a) $\alpha \mathrm{Vs} \mathrm{Cl}$

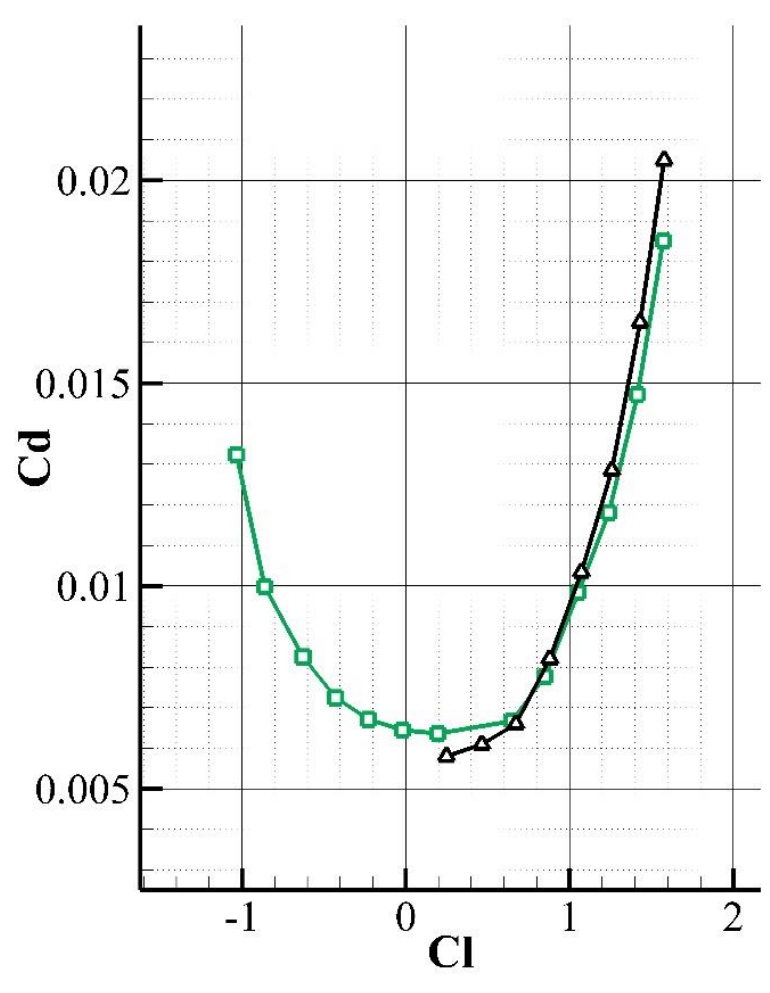

(b) $\mathrm{Cl} \mathrm{Vs} \mathrm{Cd}$

Figure 2.13. Comparison between the CFD and the experimental values [44] of (a) the lift coefficient $\mathrm{Cl}$ at different angle of attacks $\alpha$ and (b) the drag coefficient $\mathrm{Cd}$ at different lift coefficient $\mathrm{Cl}$ values.

A more sensitive aerodynamic parameter is the drag coefficient at different lift coefficient values. This property is very sensitive to the surface roughness and the free stream turbulent intensity. Tuning of the airfoil surface roughness and main stream turbulent intensity was essential to achieve the agreement shown in figure $(2.13 \mathrm{~b})$.

This validation test case provided the needed confidence to proceed with this CFD tool to study the aerodynamics of different morphing skins. 


\section{Flow OVER TRENCHES OF SLIDING PANELS}

\subsection{Introduction}

The research topic of viscous flow over rectangular cavities has recently attracted the interest of many researchers studying fluid dynamics, aeronautics, and airframe noise control. The geometry of a rectangular cavity over which viscous flow is travelling has practical implications in modeling flows over airframe discontinuities such as slotted flaps and slats, engines gaps, landing gear and weapon bays, and skin discontinuities. The high-speed flows interact with the structure of these discontinuities creating highly unsteady flow dynamics. Such flows are usually accompanied with acoustic radiations, and hydrodynamic resonance that threatens the stability and integrity of the aircraft structure and devices [47 - 49].

Despite having a very simple geometry, flow over cavities is considered one of the rich and complex flow dynamic problems. Based on the boundary conditions, the flow may include shear layer separation, shock waves, entrapped vortices, secondary flows, boundary layer transition and flow-acoustic resonance. The latter phenomenon of hydrodynamic resonance has intrigued the interest of researchers to explore the interaction between the separated shear layer and the cavity edges in all the three common methods, namely, experimentally, analytically and numerically.

Roshko [50] and Karamcheti [51] in 1955 were among the pioneers who studied this flow phenomenon. Roshko showed through his wind-tunnel experimental results [50] that sound waves are emitted from the pressure stagnation point located at the cavity trailing edge. The upstream directivity of these acoustic waves at moderate to supersonic Mach numbers was confirmed visually by Karamcheti [51] through the shadowgraph images captured from his wind-tunnel experiments. The details of this phenomenon were first revealed by Rossiter [52] who managed to relate the frequency of the acoustic radiations to the aspect ratio of the cavity and Mach number of the flow through a semi-empirical formula. The mode of instability explored by Rossiter is sometimes referred to in literature as Rossiter mode or shear layer mode. In this mode, the KelvinHelmholtz instability amplifies the small disturbances found in the shear layer over the cavity and rolling up of the vortices that travel downstream and hit the trailing edge of the cavity. The impingement of the vortices at the trailing edge of the cavity causes acoustic radiations to be emitted from the cavity trailing edge and travels upstream to close the feedback process that further 
amplifies the shear layer instability. The acoustic radiation frequency resonates with the vortex shedding frequency in a state known as the self-sustained oscillations. Sarohia [53] recorded that the onset of oscillations is observed when $\sqrt{R e_{\theta}} L / \theta_{0}>800$, where $R e_{\theta}$ is Reynolds number based on the momentum thickness, $L$ is the length of the cavity and $\theta_{0}$ is the momentum thickness of the boundary layer at the leading edge of the cavity. Gharib and Roshko [54] confirmed this finding where they observed the onset of oscillations at $\sqrt{R e_{\theta}} L / \theta_{0} \approx 720$, a value that is close enough to the value obtained by Sarohia [53]. Below this value, the flow was observed to be steady in nature. A more substantial result documented by Gharib and Roshko [54] was a new instability mode know as the wake mode. In the wake mode, the vortex entrapped in the cavity starts to emerge out of the cavity resulting in amplified off-wall oscillations in the velocity field surrounding the cavity. The oscillations observed in the wake mode resembles the wake oscillating flow behind bluff bodies, and hence the name.

With the recent advancements of computational power, and the high fidelity achieved by CFD codes and numerical algorithms, researchers started to use this new approach to explore different modes of oscillations, investigate the physical details of this flow while avoiding the external effects of intrusive experimental tools.

Colonius et al. [55, 56] and Rowley et al. [57] captured the three modes of oscillations, namely, no-oscillations mode, shear layer mode, and wake mode using two-dimensional direct numerical simulation (DNS). Rizzetta and Visbal [58], Larchevêque et al. [59 - 61], and Chang et al. [62] reached an agreement between results of the three-dimensional large-eddy simulations (LES) and the experimental values of Rossiter's frequencies. Since flow over cavities is characterized by large coherent turbulent structures, some researchers studied the flow phenomena using the unsteady RANS simulations at different flow regimes; from subsonic [47, 63 - 65], to supersonic regimes [64, $66-72]$. Over years, the complexity of the turbulence model used in the RANS method has developed from a single algebraic equation, to two equations $k-\varepsilon$ and $k-\omega$ turbulence models with their main objective being to reproduce the frequencies of oscillation obtained from experiments and Rossiter's formula.

As shown in chapter 2, the transitional SST turbulence model has proven to be an accurate enough to model the boundary layer transition. In this chapter, the URANS equations will be used with the four-equation transitional SST turbulence model to find an approximate scaling law that can predict the transition of the boundary layer over cavities. This scaling law can then be used in designing the rectangular cavity between adjacent sliding panels of a sliding morphing skin. This approach can result in a morphing airfoil that preserves the aerodynamic properties of a clean airfoil. Section 3.2 presents a numerical verification of the numerical results obtained, and a numerical validation of the results by comparing them qualitatively and quantitatively to experimental and semi-empirical results. The good accuracy achieved by the numerical results has 
encouraged a more detailed parametric study in section 3.3. In this section, the correlation between different non-dimensional numbers and the state of the boundary layer is studied. The result of this analysis will be a scaling law that can predict the transition of the boundary layer. Section 3.4 presents the practical implications of this finding with a focus on morphing skin applications. The scaling law can be used to optimize the skin design and ensure optimal aerodynamic performance that is comparable to the clean airfoil. It should be noted here that the cavity depth was not accounted for in this study because the cavity is assumed to be infinitely deep compared to the shear layer thickness [57]. 


\subsection{Numerical Model Validation}

\subsubsection{Flow Configuration}

To investigate the geometric parameters that influence the behavior of the flow over 2D cavities, several geometric dimensions are being varied in the following study. Figure (3.1) shows the computational domain with its geometric nomenclature that will be used in the rest of this chapter. To cover a wide spectrum of flow conditions over the cavity, the flow parameters as well as the geometric properties vary over a wide range. The leading edge of the plate changed over the range of $X / D=5$ to $X / D=25$, and the width of the cavity itself changed from $L / D=0.5$ to $\mathrm{L} / \mathrm{D}=4$, where $\mathrm{D}$ is the depth of the cavity, $\mathrm{X}$ is the leading-edge length of the flat plate before the cavity, and $\mathrm{L}$ is the width of the cavity. The freestream Mach number changed over the range of 0.2 to 0.8. This combination of parameters has resulted in a non-dimensional boundary layer momentum thickness at the leading edge of the cavity ranging from $L / \theta_{0}=10$ to 200 , and a Reynolds momentum thickness at the leading edge of the cavity that is ranging between $R e_{\theta_{0}}=95$ and 1500 . The combination of all these parameters has resulted in more than 75 numerical cases. In all cases the depth of the cavity $\mathrm{D}$ is set to $1 \mathrm{~mm}$. The height $\mathrm{Y}$ and the width $\mathrm{W}$ of the computational domain are determined after examining the effect of the computational domain size on the flow features and the acoustic radiations.

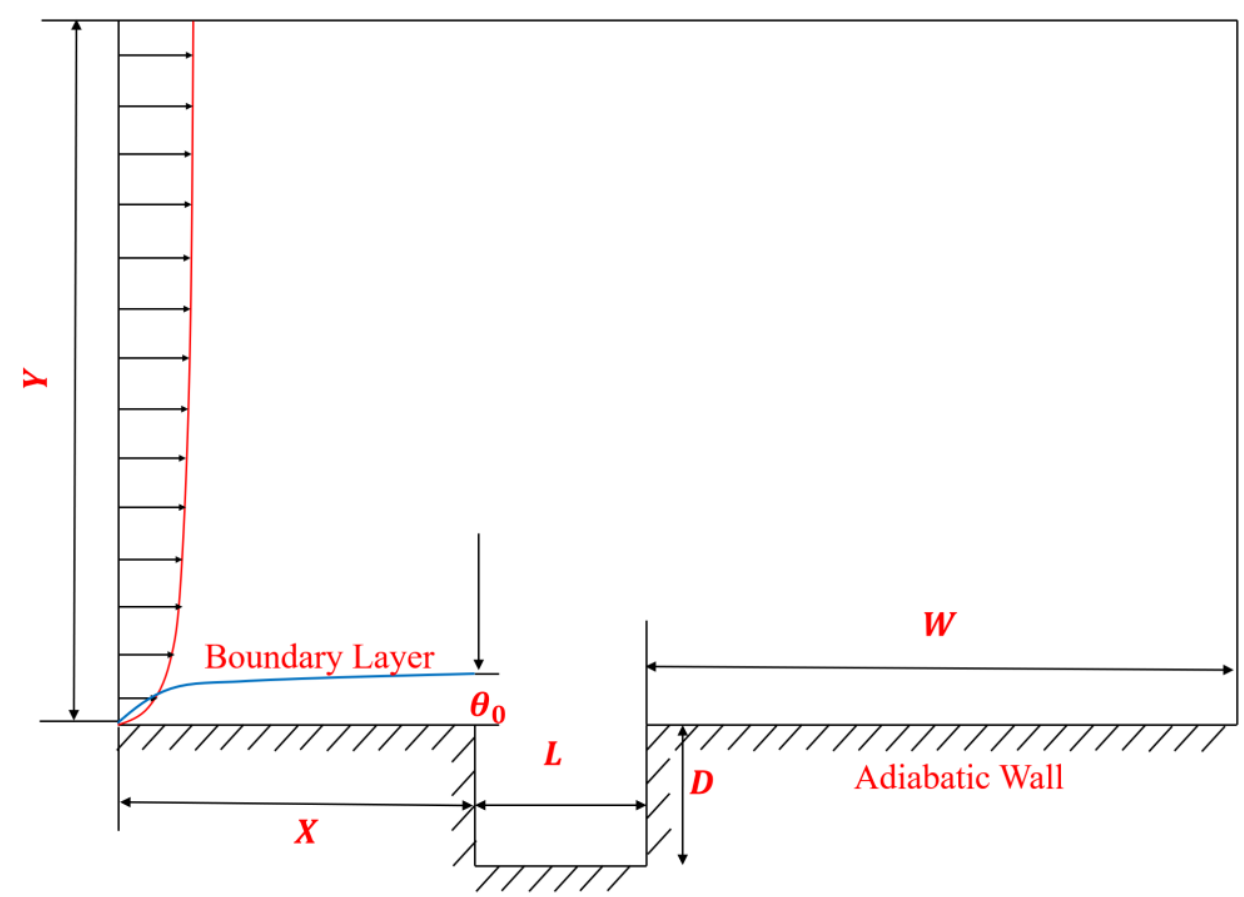

Figure 3.1. A schematic diagram of the cavity computational domain and the used nomenclature. 


\subsubsection{Boundary Conditions and Computational Domain}

The type of the boundary condition and the size of the computational domain can significantly influence the results of CFD analysis, so it is of a great importance in such CFD cases to ensure the independence of the solution from the computational domain size, and to choose the proper non-reflecting boundary conditions. For that reason, a pressure far-field boundary condition is used for the three non-wall boundaries, namely, the upper, left and right boundaries. By applying the Riemann invariants for the 1D flow normal to the boundary at the pressure far-field conditions, acoustic and pressure waves are not reflected over these boundaries. All wall conditions at the leading and trailing flat plates, and the walls of the cavity are treated as no-slip adiabatic walls. The size of the computational domain was determined after varying the computational domain size and monitored the $y$-velocity fluctuations at $\mathrm{x}=3 \mathrm{D}$ and $\mathrm{y}=0$ measured from the leading edge upper corner of the cavity. The height $\mathrm{Y}$ and width $\mathrm{W}$ of the computational domain were both set to $12.5 \mathrm{D}$ for the first case, $25 \mathrm{D}$ for the second case and 50D for the last case. The cavity length to depth ratio $L / D$ is set to 4 and the length of the leading edge of the flat plate is set to $X / D=5$. The freestream Mach number is 0.6. Such a combination has resulted in a boundary layer momentum thickness ratio $L / \theta_{0}$ of 194 and Reynolds momentum thickness at the leading edge of the cavity of $R e_{\theta_{0}}=282.4$. Figure (3.2) shows the measured y-velocity fluctuations on the y-axis, and on the $\mathrm{x}$-axis the time is presented after being normalized by the freestream velocity and the cavity depth.

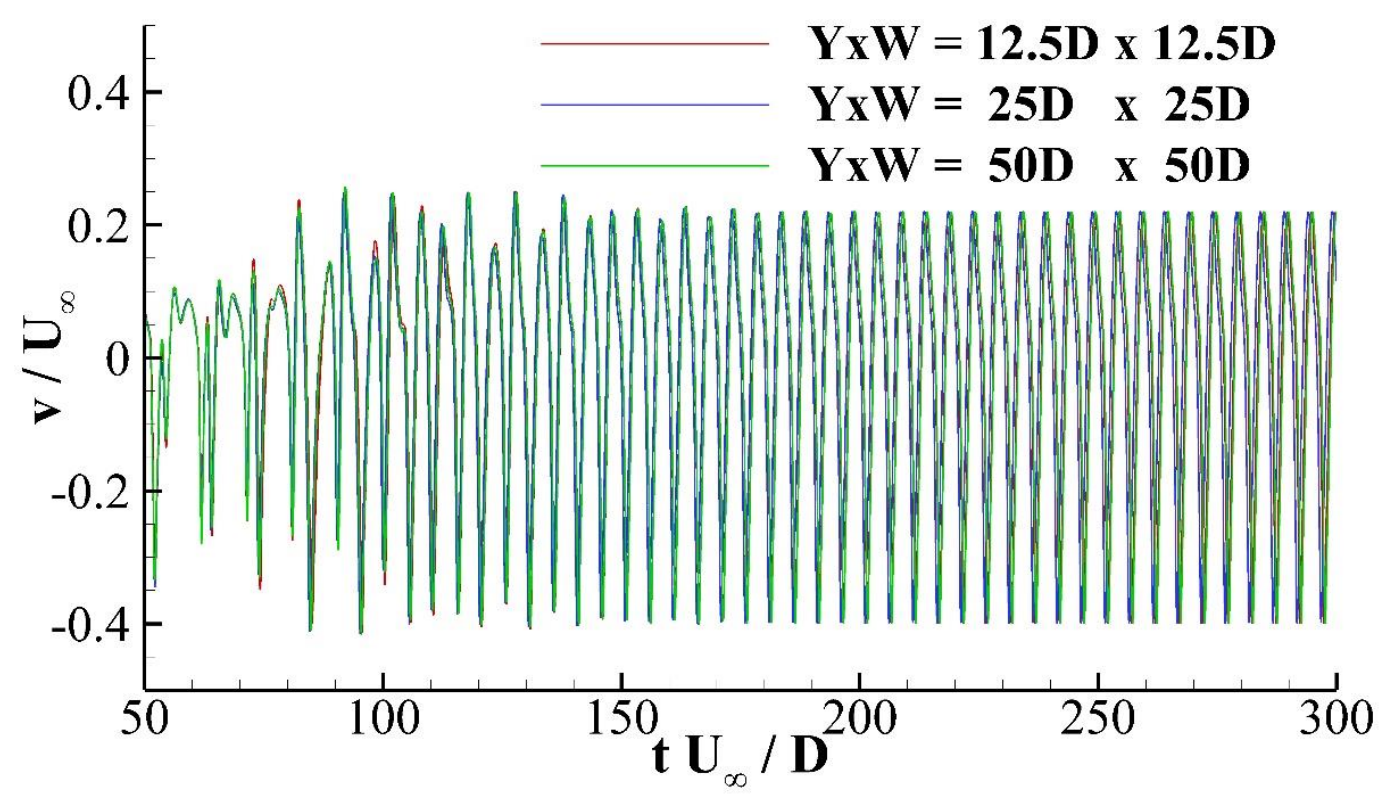

Figure 3.2. Y-component of the velocity fluctuations measured in three computational domains with different sizes. 
As shown in figure (3.2), results of the three domains are nearly coinciding with minor discrepancies observed in the first case at the first 100 time-units. This discrepancy is observed as slightly higher amplitudes at the peaks that quickly attenuates after the first 100 time-units. For that reason, the second size of the computational domain has been chosen to be used for the rest of this chapter with the height and width of the computational domain being $\mathrm{YxW}=25 \mathrm{D} \times 25 \mathrm{D}$.

\subsubsection{Meshing and Spatial Convergence}

Creating a mesh that resolves the complex flow phenomena using URANS equations is a challenging task. The grid has to properly resolve the viscous boundary layer attached to the leading and trailing plates, at the same time it has to have as much regular elements over the cavity vicinity as possible. It was found from numerical simulations that if the mesh cells over the cavity have high aspect ratio, they produce an unphysical pressure field, and fail to capture the interaction of the acoustic radiations with the vortex shedding. For these reasons, the computational domain has been divided into 14 different blocks as shown in figure (3.3).

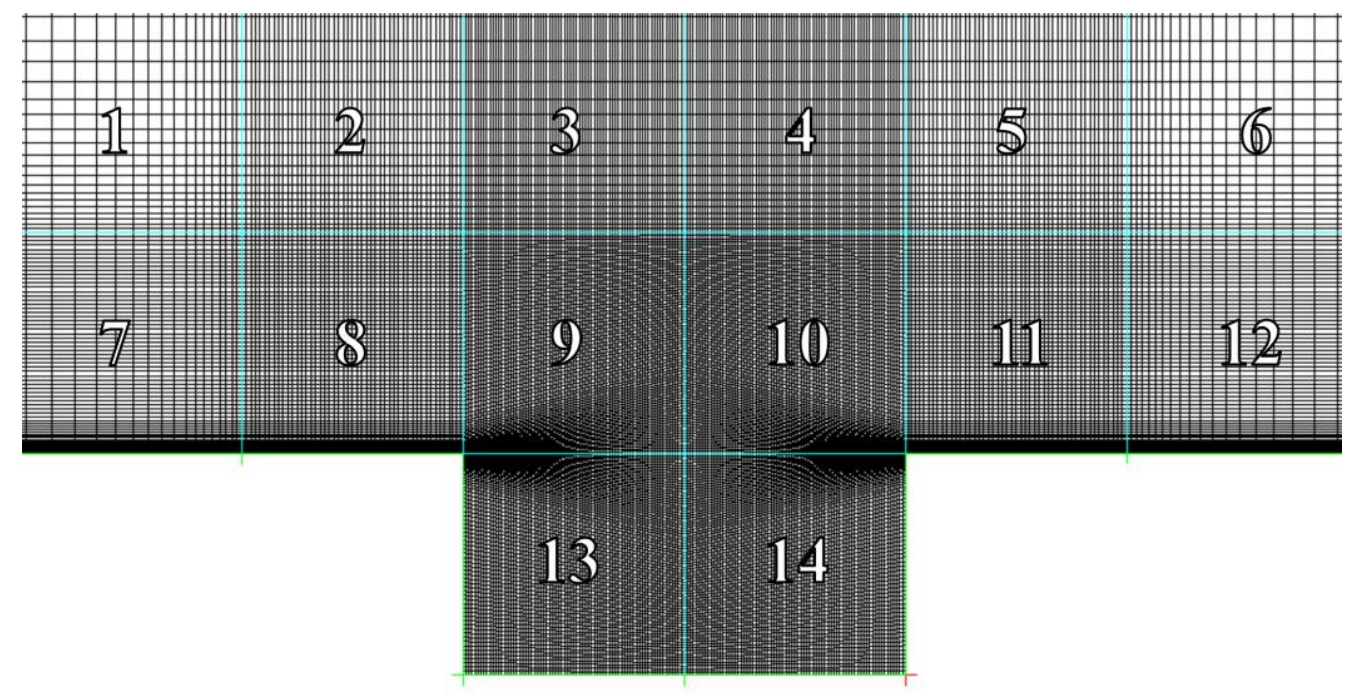

Figure 3.3. The blocking and mesh configurations used to resolve the flow over cavities.

The off-wall nodes have been clustered near the walls of blocks 7, 8, 11 and 12 to properly resolve the velocity gradient of the viscous boundary layer at the flat plate. Blocks 8 and 11 blend gradually with blocks 9, 10, 13 and 14 to expand the closely clustered nodes near the walls and reach a uniform distribution at the common edges between blocks 9 and 10, and blocks 13 and 14. This ensures that the aspect ratios of cells over the cavity have values closer to unity. The nodes in blocks $1,2,3,4,5,6,7$, and 12 expand gradually along the $\mathrm{x}$ and $\mathrm{y}$ directions towards the pressure 
far-field boundaries with a growth rate of 1.1. The maximum dimension in the whole mesh has been limited to 0.1 in case of the fine mesh and 0.22 in case of the moderately dense mesh. At all flow conditions, the $\mathrm{Y}+$ value is far below 1 .

The second challenge faced in the mesh generation process is choosing the optimal mesh density that compromises between capturing all the important flow feature and keeping the computational requirement to minimum. To achieve this optimal mesh density, the procedure explained in section 2.1 will be used here where the flow will be solved on a family of three consecutively refined meshes; coarse, medium and fine. A refinement factor of 2 was chosen, which means that the number of nodes are doubled in each direction, and quadrupled from one mesh to the other. The coarse, medium and fine meshes have approximately 38,750, 155,000 and 620,000 nodes respectively. The flow feature that is being tracked for the Richardson's extrapolation method is the non-dimensional Strouhal number $S t_{D}$ of the vortex shedding based on cavity depth and freestream velocity. The non-dimensional Strouhal number $S t_{D}$ is calculated from the y-velocity fluctuations at $\mathrm{x}=3 \mathrm{D}$ and $\mathrm{y}=0$ measured from the upper left corner of the cavity. This value has been chosen due to the essential role it is playing in the validation process of the numerical results versus the experimental and the semi-empirical equations.

For this numerical verification case, the cavity aspect ratio was set to $L / D=4$, a freestream Mach number of 0.8 and a leading edge of the flat plate of 5D. This configuration is known from literature to result in 'wake mode' fluctuations. This mode of oscillations is characterized by its vortex shedding frequency that is independent of the freestream Mach number, and that the velocity fluctuations over the cavity have a double peak spectrum. Further description of this oscillation mode will be presented in section 3.3.

The continuum value $\mathcal{F}_{h \rightarrow 0}$ of the fundamental frequency was calculated using equations (2.1) and (2.2) to be 0.048367 as shown in figure (3.4).

The values $\mathcal{F}_{C}, \mathcal{F}_{M}$ and $\mathcal{F}_{F}$ were calculated to be equal to $0.1983,0.0562$ and 0.0487 , respectively. The percentage difference between the values $\mathcal{F}_{C}, \mathcal{F}_{M}$ and $\mathcal{F}_{F}$ compared to the continuum value $\mathcal{F}_{h \rightarrow 0}$ are $310 \%, 16.3 \%$ and $0.86 \%$, respectively. The fine mesh is closer to the continuum value, however, it consumed a significant amount of computational power that will limit the diversity of this study. For that reason, the medium mesh has been chosen to be used in the rest of this chapter. 


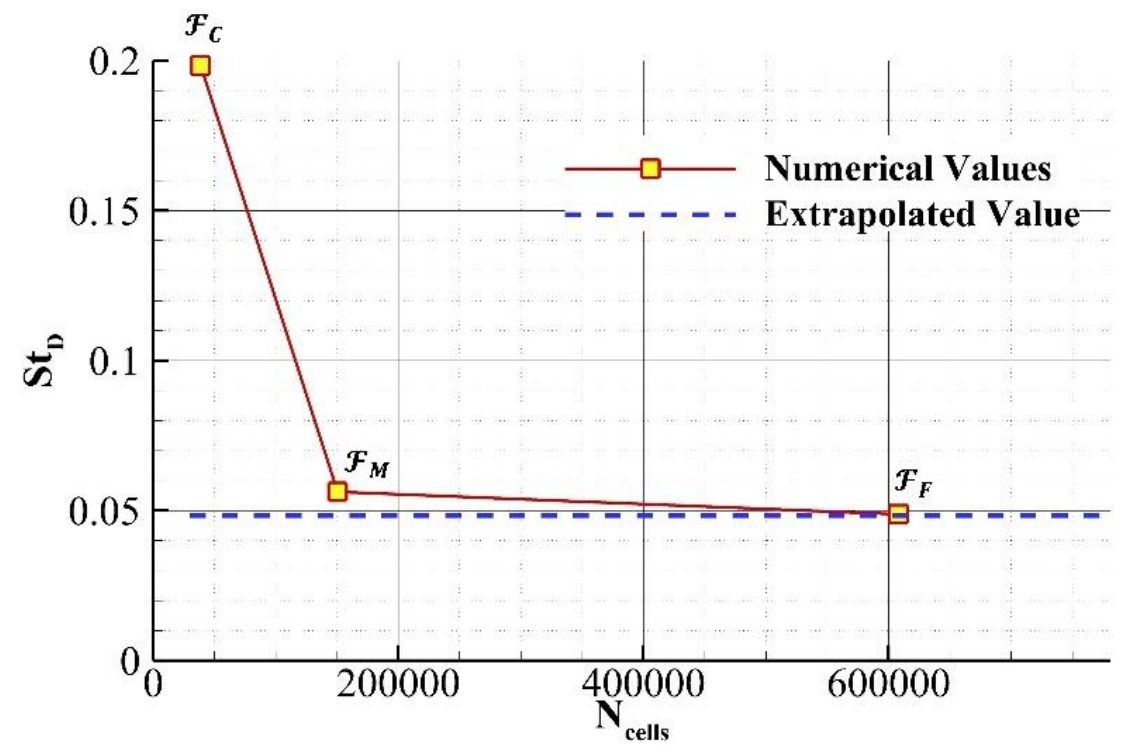

Figure 3.4. Spatial convergence of the fundamental frequency towards the asymptotic extrapolated value as the number of elements in the mesh increases.

It should be noted here that the coarse mesh in this study failed to capture the double peak oscillations that characterize the 'wake mode' oscillations. This shows that there is a threshold value in the spatial discretization that has to be met in order to capture the correct physics of this mode. Figure (3.5) shows a comparison between the y-velocity fluctuations obtained from the three meshes.

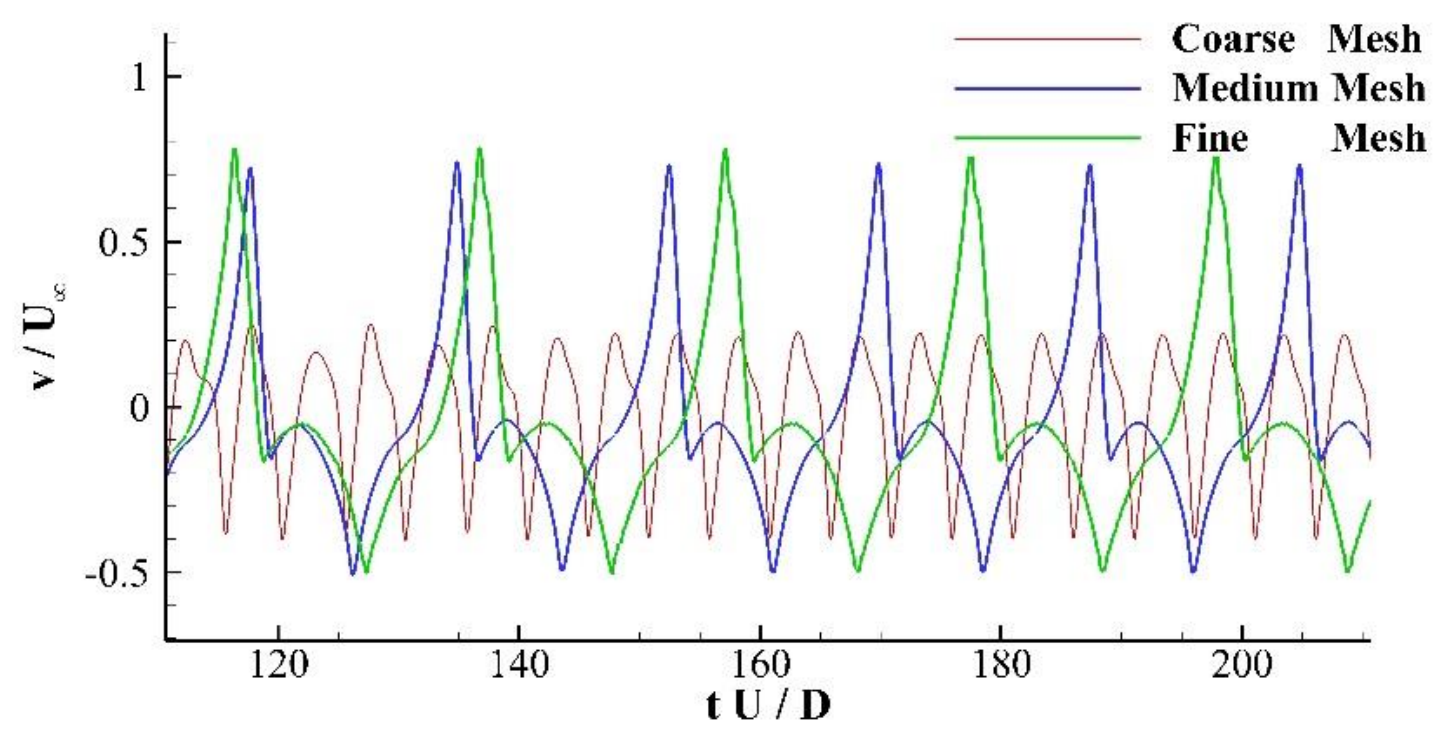

Figure 3.5. Comparison between the y-velocity oscillations in a wake mode flow on three consecutively refined meshes. 
As shown in figure (3.5), the coarse mesh failed to capture the double peak oscillations that have been correctly captured by the medium and fine meshes. In addition, it can be observed from figure (3.5) that the magnitude of the oscillations is suppressed in the case of the coarse mesh at a magnitude of 0.22 . This is compared to 0.74 and 0.78 in cases of the medium and fine meshes, respectively. This shows that there is a minimum resolution for the meshes that can correctly capture the interaction of the acoustic radiations with the other flow features.

\subsubsection{Temporal Convergence Accuracy}

A second-order temporal discretization scheme is used to capture the highly unsteady nature of flows over cavities whose vortex shedding and acoustic radiations are characterized by their high frequency interactions. This means that the time step $\Delta t$ has to be small enough to ensure a timestep independent solution. To find the largest time step that guarantees a time-step independent solution, a single cavity flow case was solved using four significantly different time-steps of $\Delta t U / D=5.2,0.52,0.052,0.0052$. The $\mathrm{y}$-velocity at $\mathrm{x}=3 \mathrm{D}$, and $\mathrm{y}=0$, measured from the upper left corner of the cavity has been monitored in each case and plotted in figure (3.6).

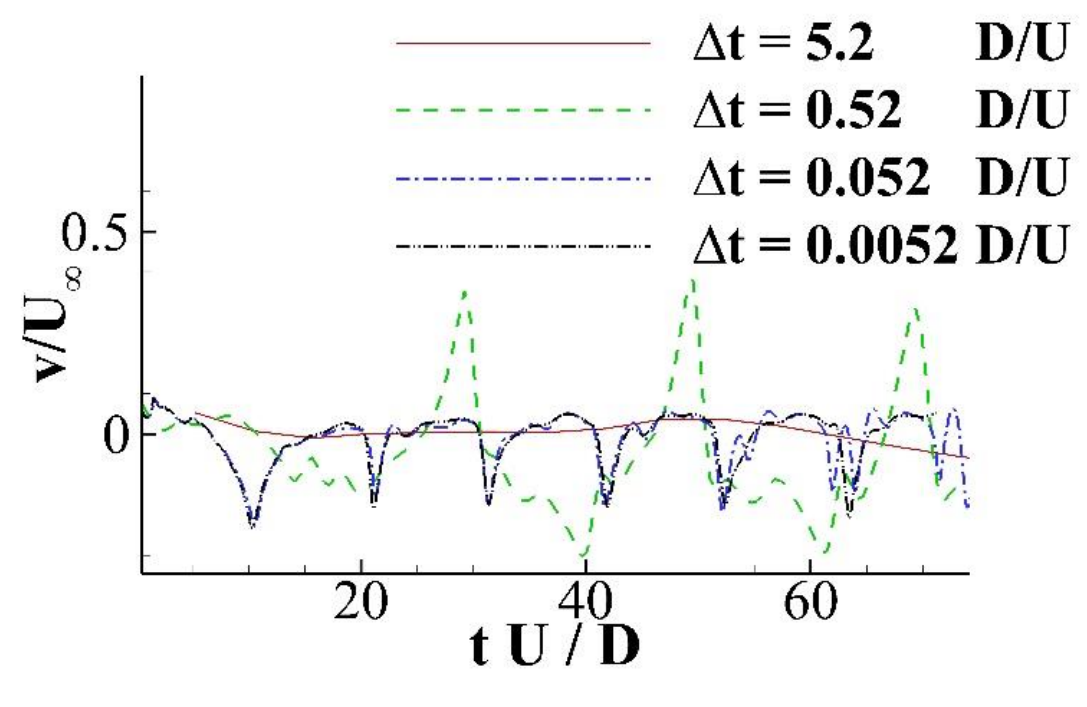

Figure 3.6. Y-velocity fluctuations over a cavity obtained using four different time-steps of $\Delta t U / D=5.2,0.52,0.052,0.0052$.

As shown in figure (3.6), the results of the two cases with the finest time-steps $\Delta t U / D=0.052$ and 0.0052 have obtained almost identical results. While the case with a time-step of $\Delta t U / D=$ 0.52 has resulted in fluctuations that are much higher in magnitude, and the largest time-step did not manage to capture any fluctuations. For that reason, the time-step of $\Delta t U / D=0.052$ is used as the marching time-step in this chapter. 


\subsection{Validation of the Computational Model}

This section focuses on validating the numerical results by comparing them to the experimental and semi-empirical correlations, and the numerical model accuracy in resolving the three modes of oscillations will be examined. As mentioned earlier, it is documented in literature that there are three modes of oscillations, namely, no oscillations, shear-layer mode, and wake mode.

The first mode of oscillations is the 'no-oscillations' mode where the boundary layer by-passes the cavity without any oscillatory motions at small freestream velocities and/or small cavity dimensions. Woolley et al. [73] and Rowley et al. [55 - 57] observed that there is a certain threshold of shear layer disturbances that will trigger these flow oscillations. Sarohia [53] mentioned that for deep cavities, the onset of oscillations takes place when $\sqrt{R e_{\theta}} L / \theta_{0} \approx 800$. Similarly, Gharib and Roshko [54] observed that the onset of oscillations took place at $\sqrt{R e_{\theta}} L / \theta_{0} \approx 780$. The y-velocity fluctuations can easily distinguish between the three modes of oscillations. In all cases, the $y-$ velocity fluctuations were measured at nine different locations over the cavity vicinity; at $\mathrm{x}=\mathrm{L} / 4$, $\mathrm{L} / 2$ and $3 * \mathrm{~L} / 4$ and at three different elevations at $\mathrm{y}=-\mathrm{D} / 2,0$ and $\mathrm{D} / 2$ measured from the upper right corner of the cavity. The nine monitoring points obtained the same fluctuation patterns with the same frequencies, but with phase shifts. Figure (3.7) shows three different signals representing the no-oscillations, the shear layer mode and the wake mode respectively. The signals shown in figure (3.7) are the y-velocity fluctuations measured at $(3 \mathrm{D}, 0)$ from the upper right corner of the cavity for three cases, no oscillation case, shear mode case, and wake mode case.

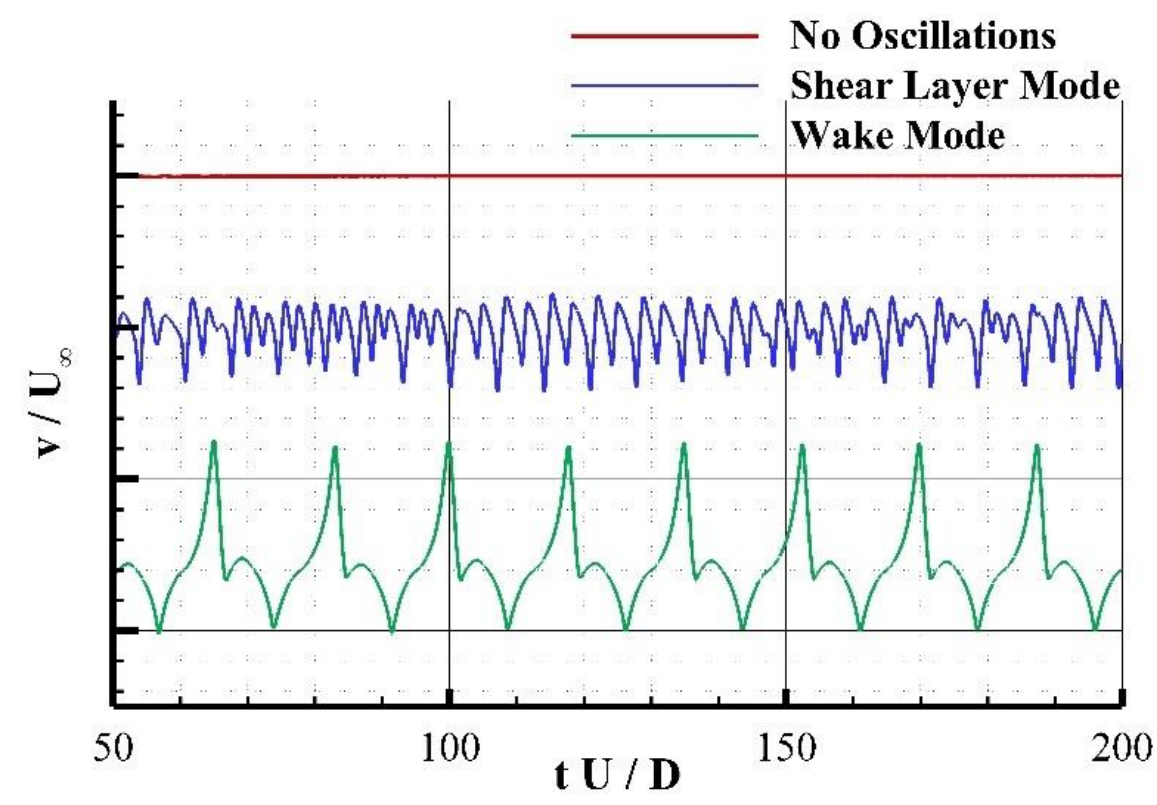

Figure 3.7. Comparison between the y-velocity oscillations in the three cavity flow regimes; nooscillations, shear layer mode, and wake mode. 
The signals are artificially shifted along the y-axis with each major tick representing $0.5 * v / U_{\infty}$, while each major tick on the $\mathrm{x}$-axis represents $50 * \mathrm{t} \mathrm{U} / \mathrm{D}$. The 'no oscillations' mode represented by the upper curve reflects a steady nature that does not oscillate. The flow is stratified and parallel to the flat plate surface. However, it was observed from the numerical simulations that a 'no oscillations' mode does not guarantee a stable state of the boundary layer. In some cases, the laminar boundary layer experienced a state transition from laminar to turbulent. While in others, the circulation of the flow inside the cavity reaches a certain limit that elevates the disturbances in the shear layer and triggers the transition of the boundary layer.

Figure (3.8) shows the intermittency contour plot of two cases, both are 'no oscillation' mode. Figure (3.8 a) shows a case with trench parameters of $L / D$ is $0.5, L / \theta_{0}$ of $19, R e_{\theta}$ of 480 and Mach number of 0.8 , while figure ( $3.8 \mathrm{~b}$ ) shows a similar case of a cavity with of $L / D$ is $1, L / \theta_{0}$ of 64 , $R e_{\theta}$ of 141 and Mach number of 0.4. It can be observed from the intermittency contour plot that despite having a lower Mach number of 0.4 , the boundary layer in figure $(3.8 \mathrm{~b})$ experienced a transition from laminar to turbulent. Further investigations of the factors that affect the transition of the boundary layer will be discussed in section 3.4.

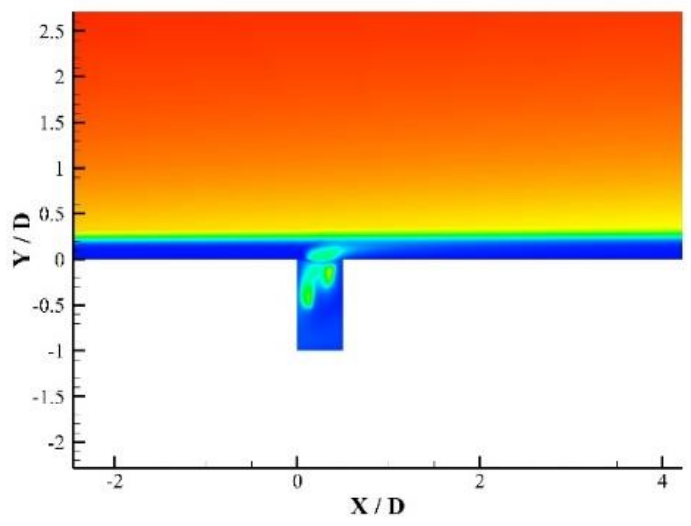

(a)

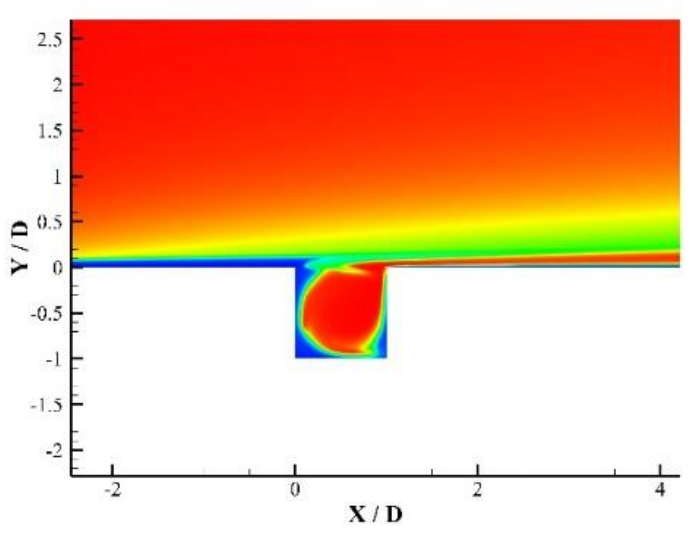

(b)

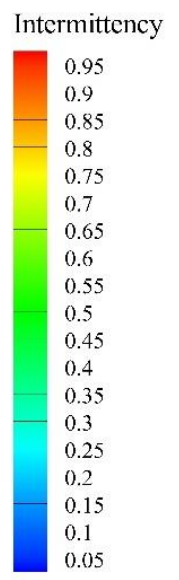

Figure 3.8. Intermittency contour plot of two cases in a cavity 'no-oscillations' mode. (a) shows no signs of boundary layer transition, while the cavity in (b) triggered the transition of the boundary layer.

The other, and more interesting mode of oscillations, is the shear layer mode, also known as, Rossiter mode. This mode is named after Rossiter [52] who was the first to study this mode of oscillations. The shear layer is characterized by a four-step feedback process shown in figure (3.9). 


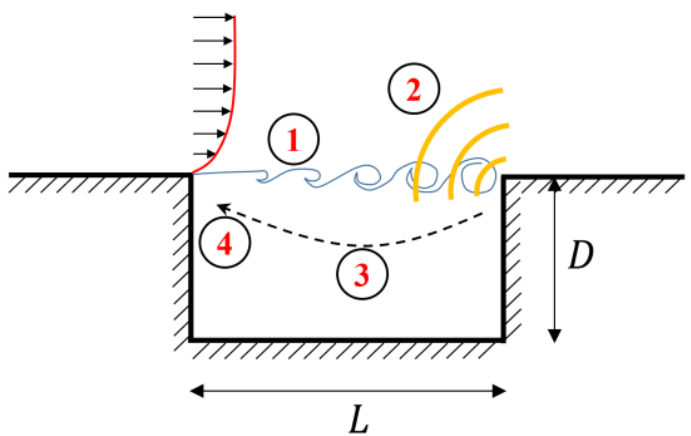

Figure 3.9. Schematic diagram of the four steps feedback process that results in the development of the shear layer mode.

As the boundary layer separates from the cavity leading edge, and due to the velocity gradient between the free shear layer and the stationary fluid inside the cavity, the Kelvin-Helmholtz instability is amplified leading to rolling up of small vortices (step 1). The vortices travel downstream and hit the trailing edge of the cavity, and this impingement results in acoustic radiations that travels upstream (step 2). The acoustic waves further amplify the Kelvin-Helmholtz instability leading to rolling up of more vortices (steps 3 and 4). This receptivity closes the loop in what is known as the self-sustained oscillations where the frequency of the acoustics resonates with the shedding frequency of the vortices.

Using the data collected from his subsonic and transonic experiments, Rossiter [52] managed to obtain a semi-empirical model that can predict the frequencies of the self-sustained oscillations at different Mach numbers as:

$$
f_{n}=\frac{U}{L} \frac{(n-\alpha)}{(M+1 / \kappa)}
$$

Another non-dimensional representation of this Rossiter formula is:

$$
S t_{n}=\frac{f_{n} L}{U}=\frac{(n-\alpha)}{(M+1 / \kappa)}
$$

Where $S t_{n}$ is the Strouhal number of the $n^{\text {th }}$ mode frequency $f_{n}, L$ is the cavity length, $\mathrm{U}$ is the freestream velocity, $\mathrm{M}$ is the freestream Mach number, $\kappa$ is an empirical constant related to the convection speed of the disturbance and $\alpha$ in another emperical constant representing a phase delay. Matching the values mentioned by Rossiter, $\kappa$ and $\alpha$ are used as 1.75 and 0.25 respectively. 
Heller and Bliss [74] modified equation (3.2) by accounting for the sound speed in the cavity based on the fluid temperature. This modified formula is:

$$
S t_{n}=\frac{f_{n} L}{U}=\frac{(n-\alpha)}{\left(M / \sqrt{1+\frac{(\gamma-1)}{2} M^{2}}\right)+1 / \kappa}
$$

Where $\gamma$ is the specific heat ratio. The results obtained from the CFD simulations showed very good agreement with the first three fundamental frequencies calculated using equation (3.3). According to equation (3.3), the non-dimensional Strouhal number of the first three modes for a Mach number of 0.8 are $S t_{1}=0.2996, S t_{2}=0.6991$ and $S t_{3}=1.0986$. Figure (3.10) shows the frequency spectrum obtained numerically for a shear layer mode with a cavity width of $\mathrm{L} / \mathrm{D}=4$, a leading-edge plate of X/D of 15, a Reynolds momentum thickness $R e_{\theta_{0}}$ of 3556.22 and $L / \theta_{0}$ of 5.13. The $\mathrm{x}$-axis is the non-dimensional Strouhal number, and the $\mathrm{y}$-axis is the power spectrum density (PSD) of the $y$-velocity component. The frequency spectrum represents the discrete Fourier transformation of the $y$-velocity fluctuations measured at $(3 \mathrm{D}, 0)$ from the upper right corner of the cavity. The semi-empirical values obtained from equation (3.3) is overlaying the numerical frequency spectrum.

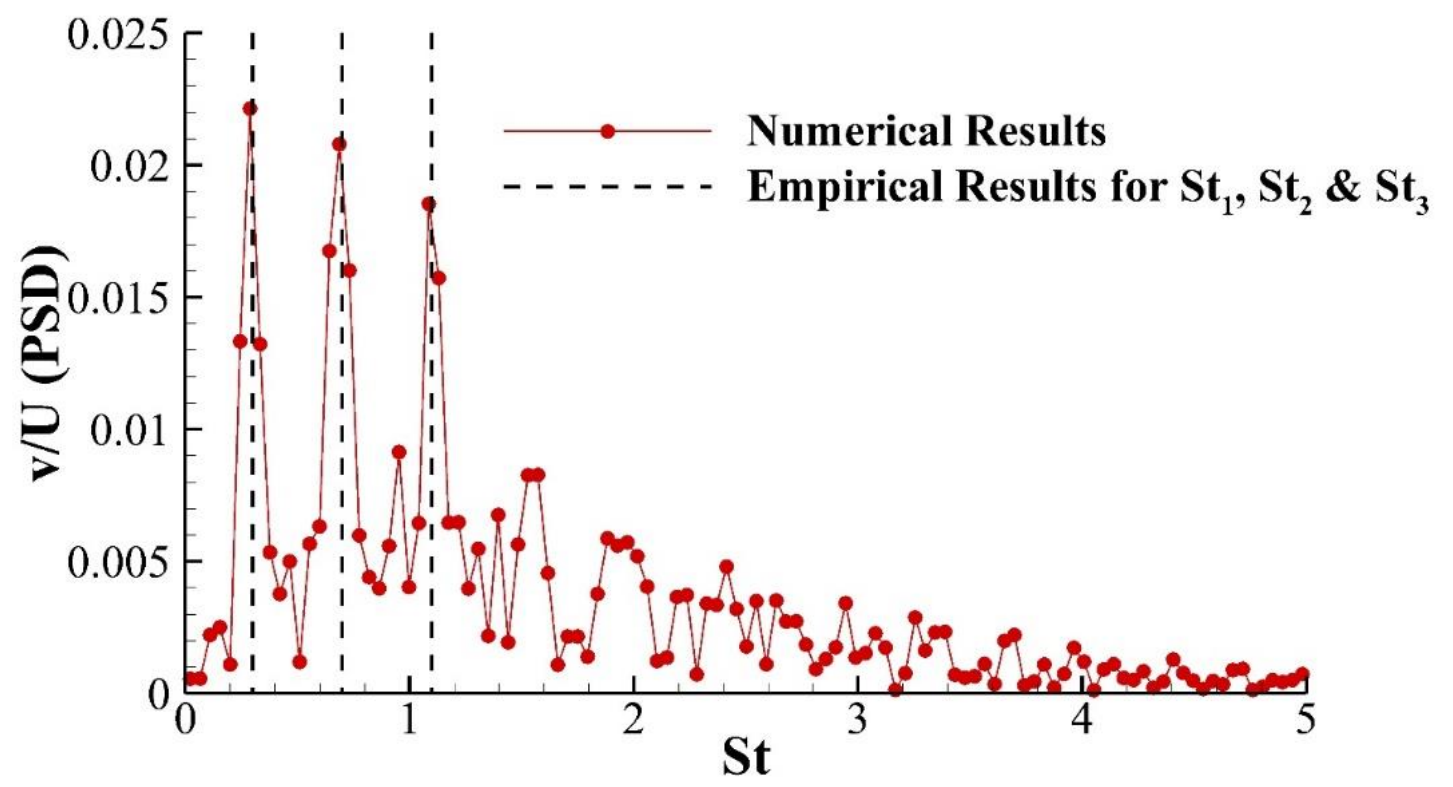

Figure 3.10. Comparison between the numerical fast Fourier transformation spectrum and the fundamental frequencies predicted by Rossiter [52] and Heller \& Bliss [74] semi-empirical equations.

As shown in figure (3.10), very good agreement can be observed between the first three fundamental frequencies calculated using equation (3.3), and the first three peaks in the DFT 
obtained numerically. Another qualitative comparison is shown in figure (3.11) where the pressure waves obtained numerically are plotted using a gray scale and compared to the Schlieren photographs experimentally captured by Krishnamurty [51] in 1956 at the same boundary conditions.

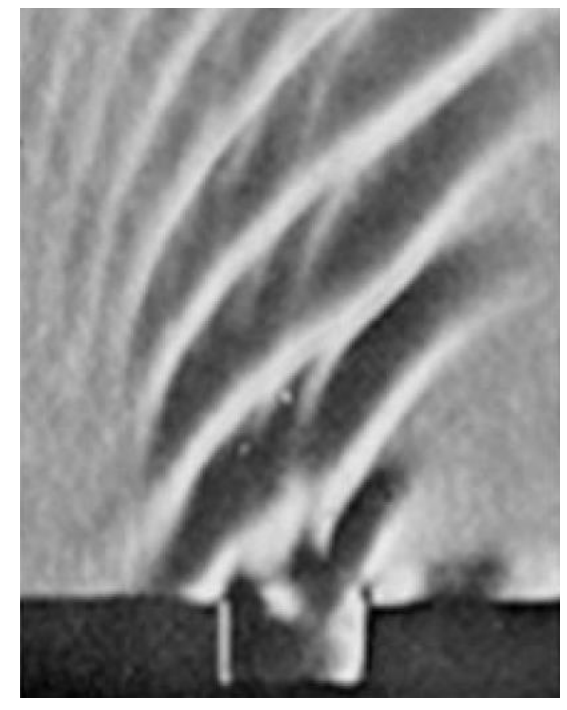

(a)

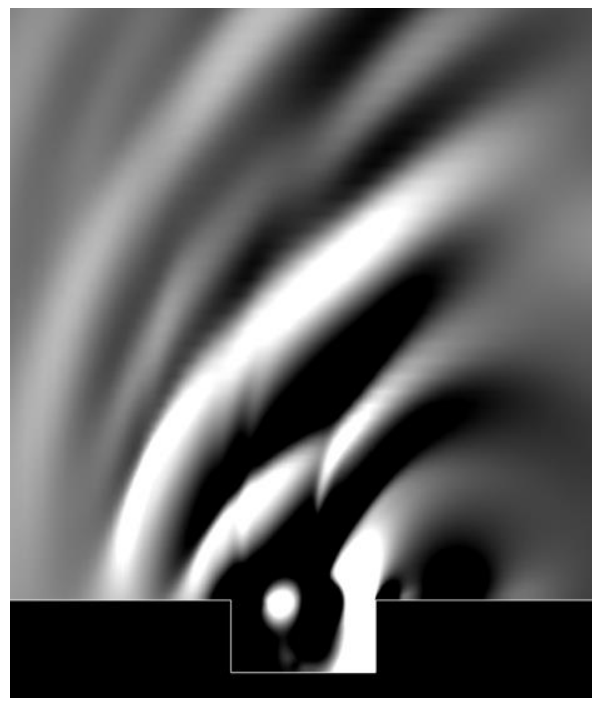

(b)

Figure 3.11. Comparison between (a) Schlieren photographs obtained experimentally by Krishnamurty [51], and (b) the results obtained from the numerical model for a Mach number of 0.8 , and a cavity with $\mathrm{L} / \mathrm{D}=2$.

Very good qualitative agreement can be observed from figure (3.11) where the experimental and numerical pressure waves are shown at the same locations and with the frequencies.

The third and last mode of oscillations is the 'wake' mode that was first documented by Gharib \& Roshko [54] in 1987. They observed that at certain flow conditions and/or cavity dimensions, the flow behavior diverts significantly from 'shear layer' mode and starts to shed large scale vortices that are comparable in size to the cavity length. They called this mode of oscillations the 'Wake Mode' because the shed vortices resemble the wakes behind bluff bodies [50]. The vortex starts its shedding from the leading edge of the cavity and travels downstream where an irrotational backflow enters the cavity from the trailing edge and travels upstream inside the cavity below the first top vortex. The first vortex is then displaced upwards by the action of the reversed flow vortex, and ejected violently from the trailing edge of the cavity. The lower reversed vortex moves up and substitutes the upper vortex, and the cycle continues. Four time-instances of the wake mode vortex shedding formation are shown in figure (3.12). 


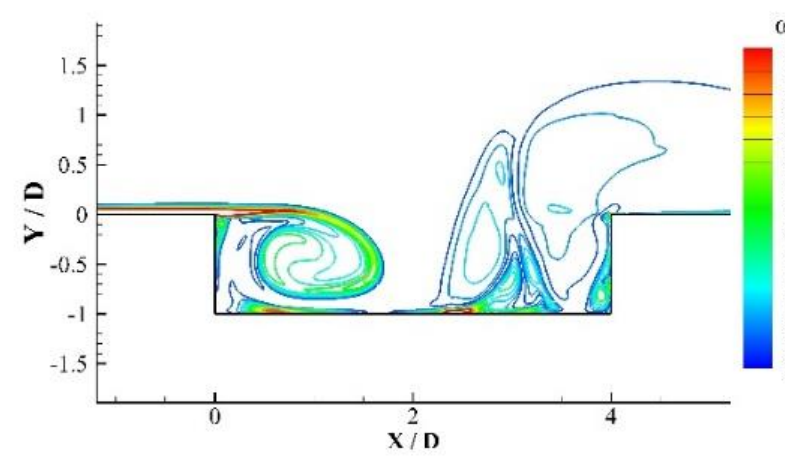

(a)

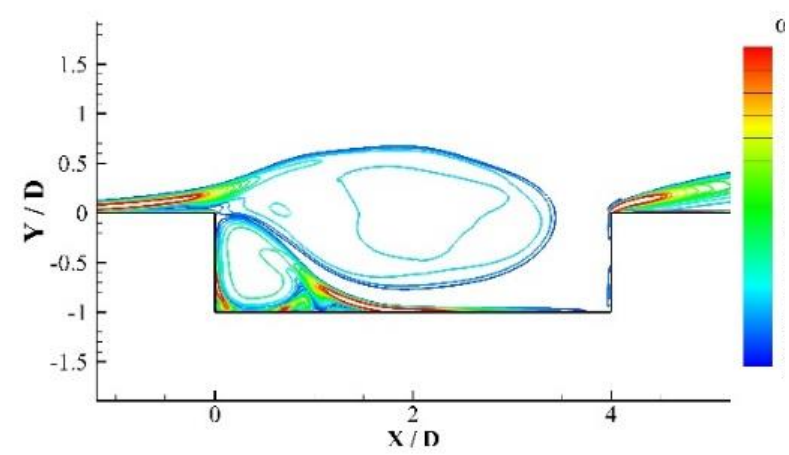

(c)

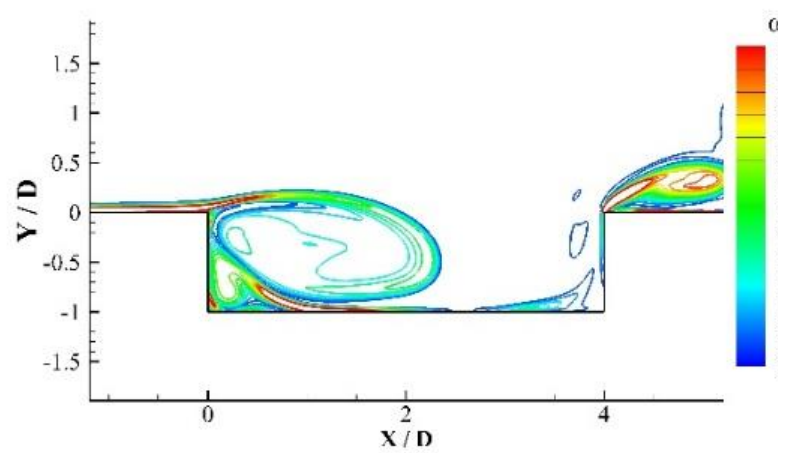

(b)

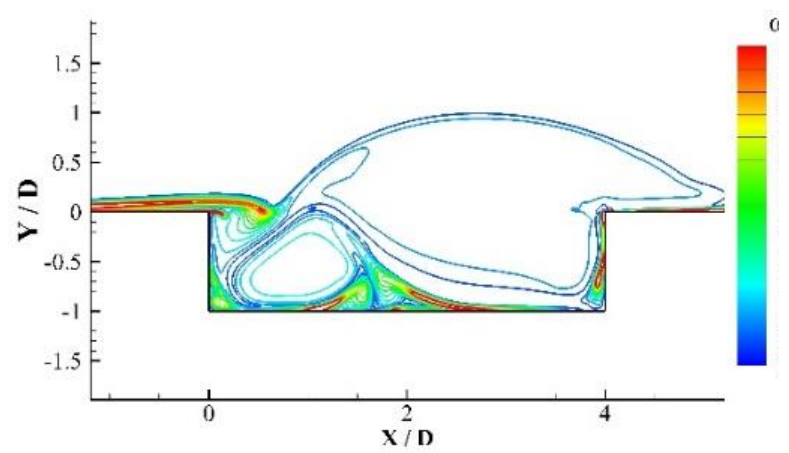

(d)

Figure 3.12. Four instantaneous contour plots of the non-dimensional vorticity magnitude showing the steps of the vortex shedding and ejection in the case of a wake mode flow at approximately (a) $\mathrm{t}=\mathrm{T} / 4$, (b) $\mathrm{t}=\mathrm{T} / 2$, (c) $\mathrm{t}=3 * \mathrm{~T} / 4$, and (d) $\mathrm{t}=\mathrm{T}$, where $\mathrm{T}$ is the periodic time of a single vortex shedding cycle.

The four snapshots shown in figure (3.12) represent a full cycle of the wake mode vortex shedding with approximately one quarter intervals of the periodic cycle of the wake formation. It is shown from figure (3.12) that the scale of the vortex shedding is the same dimension as the cavity depth. Figures (3.12 a) and (3.12 b) show that the initial vortex formation extends along the length of the cavity with a smaller vortex entrapped at the leading-edge corner of the cavity. Figure (3.12 c) shows that the major vortex emerges abruptly out of the cavity, while the smaller vortex starts moving upwards substituting the larger vortex. In figure $(3.12 \mathrm{~d})$, the flow inside the cavity is energized by the flow jet separated at the leading edge of the cavity and grows to become the primary scale vortex, and the cycle starts over again.

The frequency spectrum of the wake mode oscillations is quite different from the frequency spectrum of the shear layer mode. Even the velocity oscillations can be easily distinguished from 
the other two modes as shown in figure (3.7). The fundamental frequency is found from the numerical simulations to be independent of the Mach number. The same observation was documented by Rowley et al. [57]. In their research, they mentioned that the Strouhal frequency based on the cavity depth $S t_{D}$ is 0.064 in most cases. In a few cases where the cavity length to momentum thickness ratio increased, the independent Strouhal number was calculated as 0.054. In this research, the independent Strouhal number based on the cavity depth $S t_{D}$ was found to be 0.0571 regardless of the Mach number, cavity length, or cavity length to momentum thickness ratio. 


\subsection{Numerical Prediction of Boundary Layer Transition}

The high fidelity achieved by the URANS equations in modeling the flow details at different oscillation modes has encouraged the analysis to be upgraded to a more detailed level. In this section, the analysis will pursue a scaling law that can predict the transition of the boundary layer from a laminar to turbulent state while traveling over the cavity. Over more than 75 numerical cases, it was observed that under some conditions, the boundary layer experiences transition from laminar to turbulent, while in other cases, the boundary layer maintains its laminar state while travelling over the cavity and bypasses it as if it were a flat plate.

Several numerical cases were simulated covering a wide combination of cavity aspect ratios (L/D), leading edge momentum thickness $\theta_{0}$, and Reynolds momentum thickness $R_{\theta_{0}}$ as described in section 3.2. Different flow parameters were calculated in each case such as the boundary layer thickness $\delta$, boundary layer displacement thickness $\delta^{*}$, boundary layer momentum thickness $\theta$, shape factor of the boundary layer $\mathrm{S}$, and Reynolds momentum thickness $R e_{\theta}$. This is in addition to the fundamental non-dimensional frequencies $S t_{n}$. To study the sensitivity of the boundary layer state to different flow parameters, the following steps were followed:

a) Assess the state of the boundary layer, whether it is laminar or turbulent at the cavity leading and trailing edges. The state of the boundary layer was assessed, qualitatively and quantitatively. The quantitative assessment is based on the shape factor $\mathrm{S}$ of the boundary layer measured at the leading and trailing edges of the cavity. As mentioned by Schlichting [22], a value of 2.59 is a typical value for a laminar boundary layer, while the turbulent boundary layer shape factor ranges between 1.3 and 1.4. Since this study focuses on flow over cavities with a laminar upstream boundary layer, it was ensured that in all cases, the shape factor at the leading edge of the cavity ranged between 2.351 and 2.4666. While in the case of a boundary layer that experienced transition while traveling over the cavity, the shape factor ranged between 1.349 and 1.742. The contour plot of the intermittency function was used as a confirming qualitative assessment of the boundary layer state.

b) A matrix was created with each case listed in a separate row, and each one of the flow parameters in a separate column. The last column in the matrix is the state of the boundary layer. A value of ' 0 ' means that the boundary layer is laminar, while a value of ' 1 ' means that the boundary layer is in a turbulent state. A sample of the created matrix is shown in table 3.1 . 
Table 3.1. Sample of the matrix created to predict the factors that triggers the transition of the boundary layer over cavities.

\begin{tabular}{|c|c|c|c|c|c|c|c|c|c|c|c|}
\hline $\begin{array}{c}\text { D } \\
{[\mathrm{mm}]}\end{array}$ & L/D & X/D & $\begin{array}{c}\mathbf{M} \\
\text { Mach } \\
\text { Number }\end{array}$ & Location & $\begin{array}{c}\boldsymbol{\theta}[\mathrm{m}] \\
\begin{array}{c}\text { BL Momentum } \\
\text { thickness }\end{array} \\
\end{array}$ & $\begin{array}{c}\boldsymbol{\delta}[\mathbf{m}] \\
{ }_{\text {BL }} \\
\text { thickness }\end{array}$ & $\underset{\substack{\text { BL } \\
\text { displacement } \\
\text { thickness }}}{\boldsymbol{\delta}^{*}[\mathbf{m}]}$ & $\begin{array}{c}\mathbf{S} \\
\text { BL } \\
\text { Shape factor }\end{array}$ & $\mathbf{L} / \boldsymbol{\theta}$ & $\begin{array}{c}\boldsymbol{R} \boldsymbol{e}_{\boldsymbol{\theta}} \\
\begin{array}{c}\text { Reynolds } \\
\text { number based } \\
\text { on BL } \\
\text { momentum } \\
\text { thickness }\end{array} \\
\end{array}$ & $\begin{array}{c}\text { State } \\
\begin{array}{c}\text { State of the } \\
\text { boundary layer } \\
0=\text { Laminar }\end{array} \\
1=\text { Turbulent }\end{array}$ \\
\hline \multirow{2}{*}{1} & \multirow{2}{*}{0.5} & \multirow{2}{*}{5} & \multirow{2}{*}{0.2} & Leading edge & $2.105 e-5$ & $1.561 \mathrm{e}-4$ & $5.089 \mathrm{e}-5$ & 2.417 & 23.747 & 96.111 & 0 \\
\hline & & & & Trailing edge & $2.324 \mathrm{e}-5$ & $1.659 \mathrm{e}-4$ & $4.981 \mathrm{e}-5$ & 2.142 & 21.506 & 106.125 & 0 \\
\hline \multirow{2}{*}{1} & \multirow{2}{*}{0.5} & \multirow{2}{*}{5} & \multirow{2}{*}{0.4} & Leading edge & $1.531 \mathrm{e}-5$ & $1.171 \mathrm{e}-4$ & $3.637 \mathrm{e}-5$ & 2.374 & 32.642 & 139.841 & 0 \\
\hline & & & & Trailing edge & $1.748 \mathrm{e}-5$ & $1.317 \mathrm{e}-4$ & $3.767 \mathrm{e}-5$ & 2.142 & 28.601 & 159.604 & 0 \\
\hline \multirow[b]{2}{*}{1} & \multirow{2}{*}{0.5} & \multirow{2}{*}{5} & \multirow{2}{*}{0.6} & Leading edge & $1.285 \mathrm{e}-5$ & $1.025 \mathrm{e}-4$ & $3.035 \mathrm{e}-5$ & 2.361 & 38.898 & 176.026 & 0 \\
\hline & & & & Trailing edge & $1.521 \mathrm{e}-5$ & $1.171 \mathrm{e}-4$ & $3.021 \mathrm{e}-5$ & 1.985 & 32.856 & 208.402 & 0 \\
\hline \multirow[b]{2}{*}{1} & \multirow{2}{*}{0.5} & \multirow{2}{*}{5} & \multirow{2}{*}{0.8} & Leading edge & $1.145 \mathrm{e}-5$ & $8.791 \mathrm{e}-5$ & $2.694 \mathrm{e}-5$ & 2.351 & 43.634 & 209.231 & 0 \\
\hline & & & & Trailing edge & $1.405 e-5$ & $1.025 \mathrm{e}-4$ & $2.671 \mathrm{e}-5$ & 1.900 & 35.572 & 256.651 & 1 \\
\hline
\end{tabular}

c) Different non-dimensional quantities were generated from the matrix shown in table 3.1. These quantities combine the flow parameters with the cavity dimensions such as as $\frac{\theta}{\frac{L}{D} * \frac{L}{\theta} * R e_{\theta} * M}, \frac{\theta}{\frac{L^{2}}{D} * \frac{L}{\theta} * R e_{\theta} * \sqrt{M}}, \frac{\theta}{\frac{L}{\theta} * R e_{\theta} * M}$, and $\frac{L}{\theta} * \sqrt{R e_{\theta}}$.

d) The non-dimensional quantities obtained from step (c) were plotted on a graph with these parameters on the $\mathrm{X}$-axis, and the $\mathrm{Y}$-axis is the state of the boundary layer (' 0 ' or ' 1 ') at the trailing edge of the cavity. Among all the tested non-dimensional quantities, only one value showed a clear and sharp transition from ' 0 ' to' 1 ' at a value of approximately 600 . This non-dimensional parameter is $\frac{L}{\theta} * \sqrt{R e_{\theta}}$.

Figure (3.13) shows the strong correlation between the state of the boundary layer, and the nondimensional number $\frac{L}{\theta} * \sqrt{R e_{\theta}}$. In this figure, the state of the boundary layer is plotted on the $\mathrm{Y}$ axis, and the non-dimensional number $\frac{L}{\theta} * \sqrt{R e_{\theta}}$ is plotted on the $\mathrm{X}$-axis. The figure shows that for all cases with $\frac{L}{\theta} * \sqrt{R e_{\theta}}$ less than 600 , the state value of the boundary layer at the trailing edge of the cavity is ' 0 ' which means a laminar state. While for all cases with $\frac{L}{\theta} * \sqrt{R e_{\theta}}$ larger than 600 , the state of the boundary layer is turbulent and represented with values of ' 1 's in figure (3.13). This means that the non-dimensional number $\frac{L}{\theta} * \sqrt{R e_{\theta}}$ can be used to predict the boundary layer transition when travelling over a cavity with certain dimensions and flow properties. 


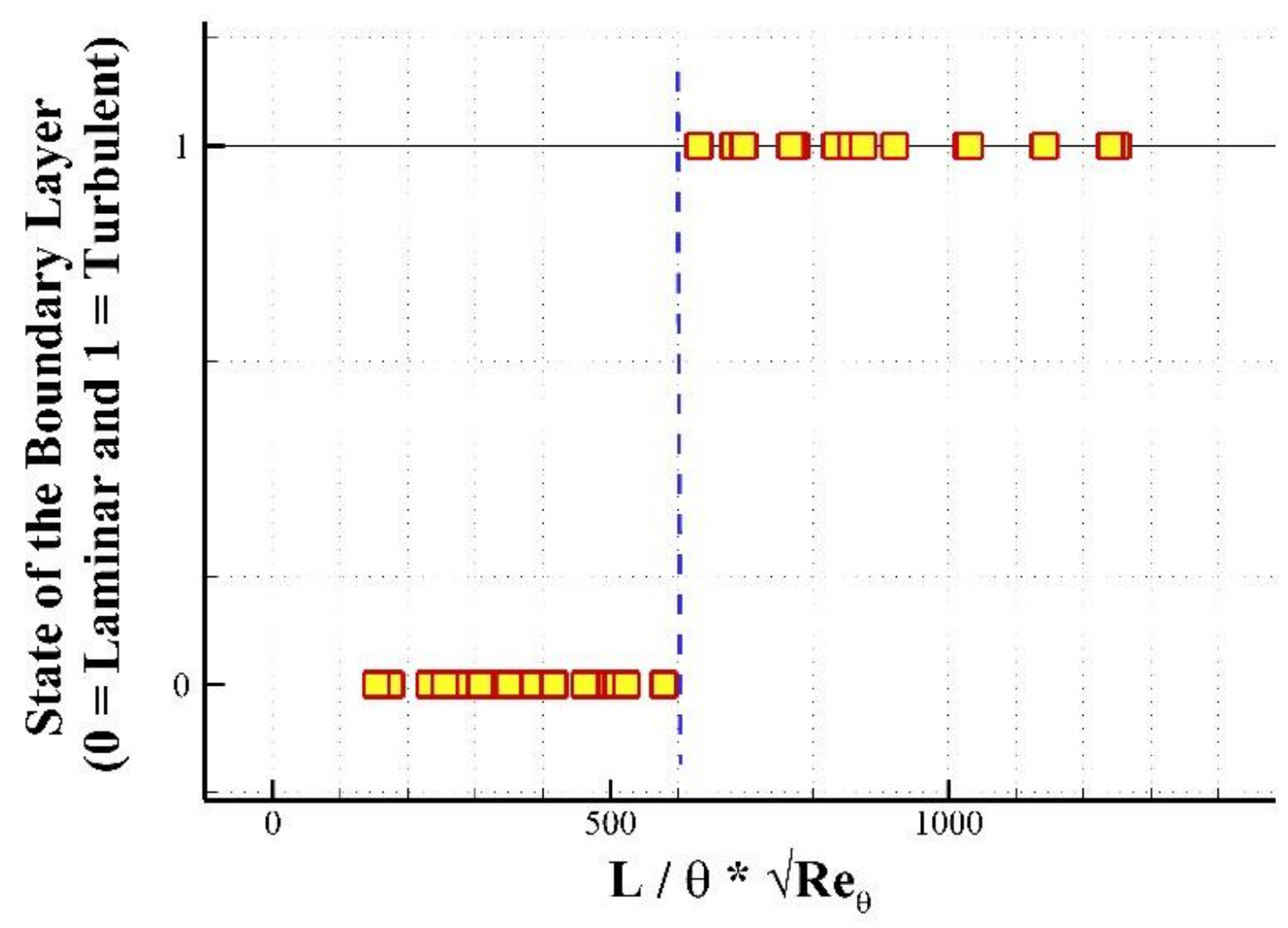

Figure 3.13. The state of the boundary layer (Laminar or Turbulent) over cavities plotted versus the non-dimensional quantity $\frac{L}{\theta} * \sqrt{R e_{\theta}}$.

To confirm this important observation, a 3D numerical case has been created of a NACA 2412 wing. The wing has a unity chord length, a unity taper ratio and no sweep angle. A trench is installed on the upper surface of the wing at $\mathrm{X} / \mathrm{C}=0.25$ and extends along the whole span of the wing which is $5 \mathrm{C}$. The depth of the trench is kept constant at $0.001 \mathrm{C}$ and its length varied along the span of the wing, starting from $0.001 \mathrm{C}$ at one end of the wing, and ends with a length of $0.005 \mathrm{C}$ at the other end of the wing. The Mach number of the mainstream flow is set to 0.2 , at atmospheric pressure and a temperature of $300 \mathrm{~K}$. The trench is small compared to wing scale and it has no sweep, no twist, no taper, therefore very little cross stream flow is expected.

The idea behind this test is to create a single case with variable $\frac{L}{\theta} * \sqrt{R e_{\theta}}$ values, instead of testing several discrete cases, each with a different $\frac{L}{\theta} * \sqrt{R e_{\theta}}$ value. As the trench length $\mathrm{L}$ varied from one side of the wing to the other, the whole non-dimensional number $\frac{L}{\theta} * \sqrt{R e_{\theta}}$ changed accordingly. By monitoring the state of the boundary layer, the sensitivity of the boundary layer to this non-dimensional number can be assessed. The state of the boundary layer was assessed by 
plotting the intermittency function at a surface that is at a very close proximity to the wing's surface. Figure (3.14) shows the results of this analysis.

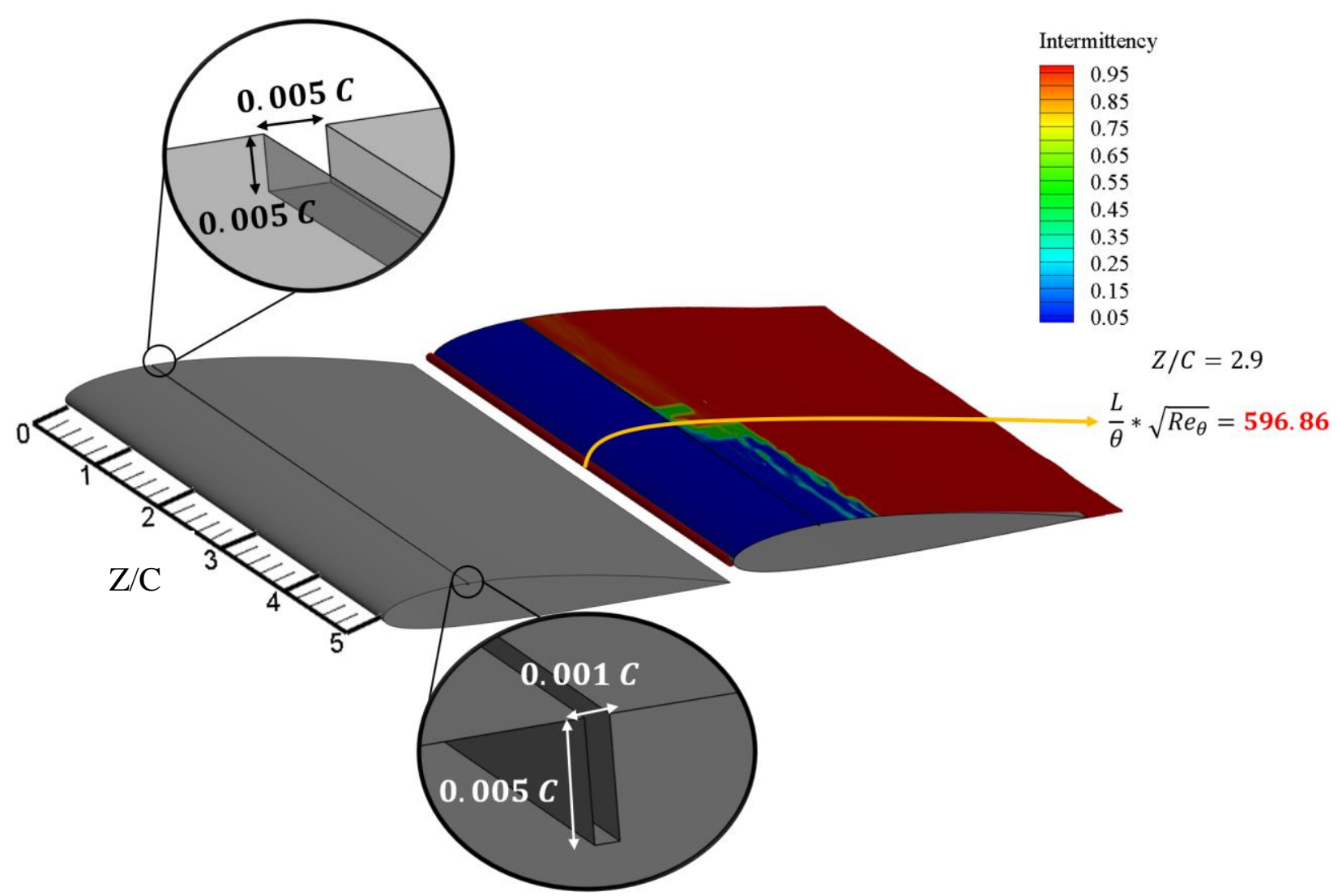

Figure 3.14. A $3 \mathrm{D}$ case of a wing with a trench having a variable length along the span. The transition of the boundary layer was shifted to the trench location at an $\frac{L}{\theta} * \sqrt{R e_{\theta}}$ value of 596.86.

Figure (3.14) shows that the transition of the boundary layer occurs naturally at approximately mid-chord length. At approximately a span position of $\mathrm{Z} / \mathrm{C}=2.9$, the transition of the boundary layer was shifted abruptly to the location of the trench which is at $0.25 \mathrm{C}$. A plane was cut at that location, and the non-dimensional number $\frac{L}{\theta} * \sqrt{R e_{\theta}}$ was calculated to confirm the observation shown in figure (3.13) with a value of 596.86. A plane was cut at $\mathrm{Z} / \mathrm{C}=3$, and $\frac{L}{\theta} * \sqrt{R e_{\theta}}$ was found equals to 582.81, and at that location the boundary layer stayed laminar over the trench. A third plane was cut at $\mathrm{Z} / \mathrm{C}=2.8$ to find a value of $\frac{L}{\theta} * \sqrt{R e_{\theta}}$ of 611.13 , and at that location, the boundary layer is totally turbulent over the cavity. These results confirm the strong correlation and dependency of the state of the boundary layer on the non-dimensional number $\frac{L}{\theta} * \sqrt{R e_{\theta}}$, and that 
the threshold value of this number is 600 . For a flow over cavity with $\frac{L}{\theta} * \sqrt{R e_{\theta}}$ less than 600 , the boundary layer will maintain its laminar state as it travels over the cavity. While in cases where $\frac{L}{\theta} * \sqrt{R e_{\theta}}$ is higher than 600 , the boundary layer will experience a transition from laminar to turbulent as it travels over the cavity. 


\subsection{Practical Applications for Morphing Skins}

The knowledge of the factors that affect the transition of the boundary layer as the flow travels over a rectangular discontinuity can have a significant effect on the design process of many aircraft parts and aerodynamic structures. For example, the gaps between the leading-edge slats and the wing can be optimized in a way that ensures an extended portion of laminar flow over the wing. Having an extended portion of the laminar boundary layer can significantly reduce the viscous drag and substantially improve the aerodynamics of the airframe. The concept is applicable on gaps found on the nacelles and on the outer skin of aircraft. As reported by Mack and McMasters [75], a $1 \%$ deduction in the drag of an aircraft can save millions of dollars per year for a typical fleet aircraft.

The recent concept of morphing wings and morphing skins can also benefit a lot from these observations. A very successful candidate of morphing skin designs is the skin of sliding panels. As it will be shown in chapter 4 and documented by Mishriky et al. [76 - 78], having a backwardfacing step on wing will significantly jeopardize its aerodynamic performance, not to mention the need of several steps to allow morphing. For that reason, the mechanical design team of morphing skins at the aerospace department at Ryerson University is trying to avoid having backward-facing steps, and instead have gaps between the panels as shown in figure (3.15). While morphing, the panels slide against each other while maintaining a certain gap distance between them. Optimizing the gap length based on the non-dimensional number $\frac{L}{\theta} * \sqrt{R e_{\theta}}$ can result in an aerodynamic performance that is similar to a clean airfoil. An example of this design is shown in figure (3.15).

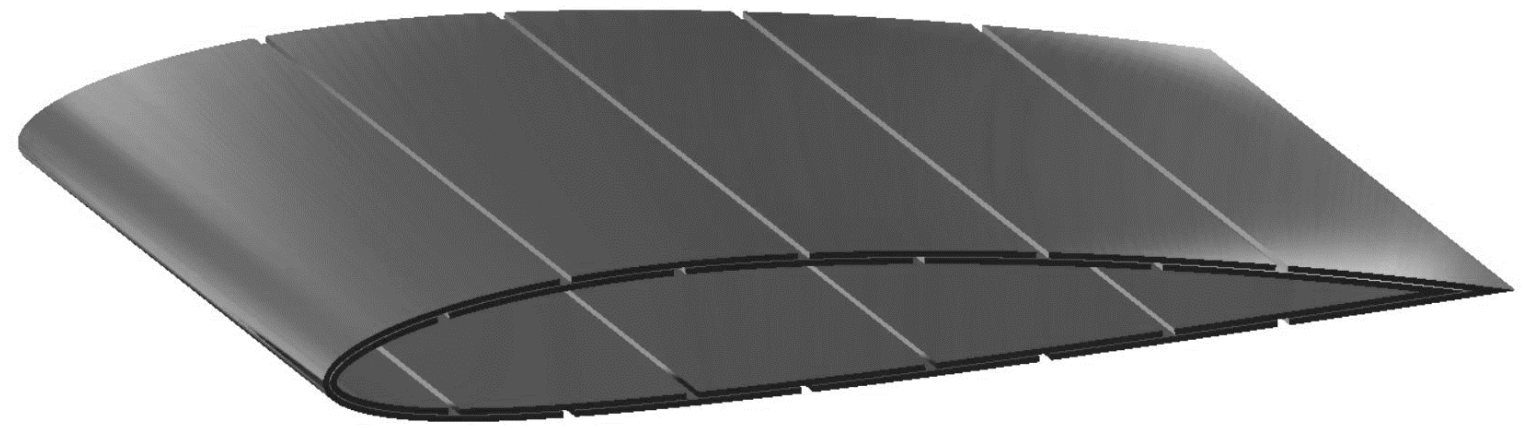

Figure 3.15. A 3D section of a morphing wing covered with sliding morphing panels. A greyscale image of figure $(1.6 \mathrm{~b})$. 
Ramrakhyani et al. [79] tested a similar design with six-segment panels using high frequency shape memory alloys (SMA). The testing was conducted in air and water tunnels to show that this design accommodated all high frequency morphing motions. Instead of randomly, or equally divided the wing into segments, Xijuan et al. [80] and Yu et al. [16 - 18] developed a more systematic way of calculating the minimum number of panels needed and the size of each panel based on the airfoil curvature while maintaining a smooth profile. Yu et al. proved mathematically that a wide range of dihedral and twist motions can be accommodated with this sliding panel design.

However, introducing a randomly designed cavity into a wing's surface can degrade the aerodynamic performance significantly, and all the potential benefits of the morphing wing concept can be lost. To emphasize this idea, a numerical test case was conducted on a unity chord length NACA 2412 airfoil with a rectangular gap installed on the upper surface of a wing at 25\% of the chord length to be approximately mid-way the laminar region of the boundary layer. Ten cases were tested, each with a different length of the gap which varied from L/C of 0.001 to 0.0075 while the depth remained constant at D/C of 0.01. Figure (3.16) shows the schematic diagram of the airfoil with a zoom on the gaps.

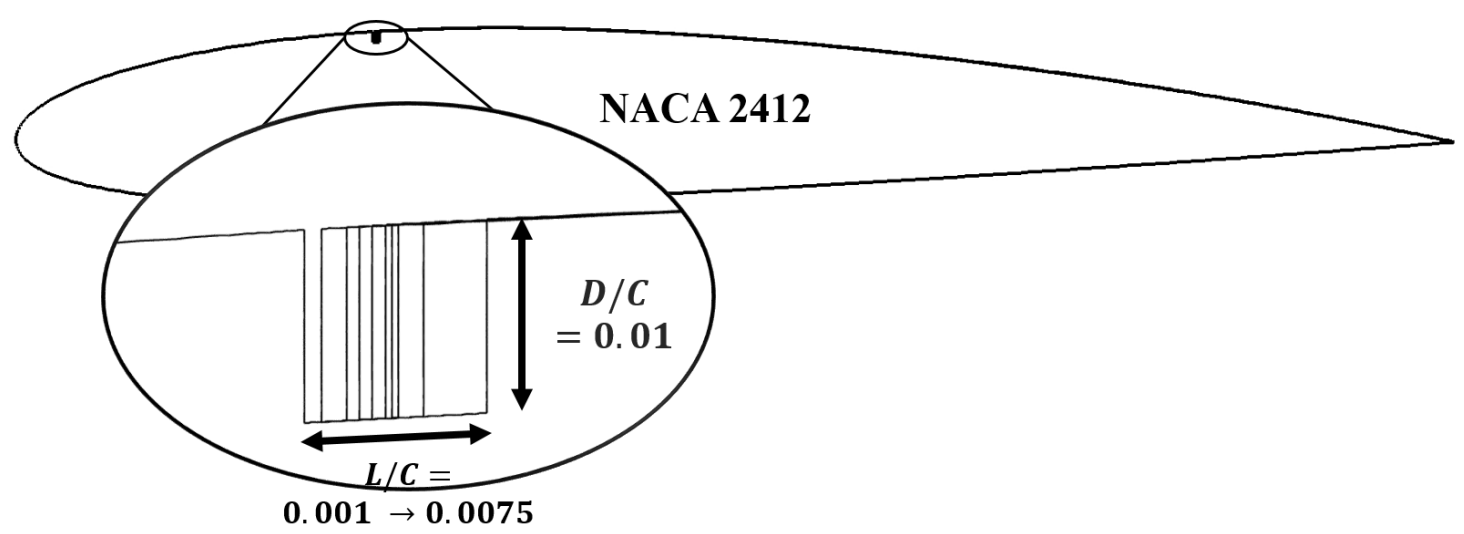

Figure 3.16. A trench introduced on the upper surface of a NACA 2412 airfoil at $X / C=0.25$ with different widths ranging from 0.001 to 0.0075 of the chord length.

The flow is set to atmospheric pressure with a temperature $300 \mathrm{~K}$ and a Reynolds number of $5.7 \mathrm{e} 6$ and an angle of attack of $2.5^{\circ}$. The same boundary conditions were used to resolve the flow over a clean airfoil. In each of the eleven cases, the drag coefficient $\mathrm{cd}$, the lift coefficient $\mathrm{cl}$, and the lift-to-drag ratio L/D has been calculated. Figure (3.17) shows the results of this analysis. 
The results in figure (3.17) show that while the gap length is small, the drag coefficient cd, the lift coefficient cl, and the lift-to-drag ratio L/D are very close to the values of the clean airfoil. This is because at small cavity lengths, the boundary layer bypasses the cavity and is treated as a solid surface similar to a clean airfoil. When the gap length increases beyond a certain threshold value, the turbulent energy inside the cavity increases and propagates to the boundary layer. This triggers the transition of the boundary layer from laminar to turbulent and degrades the aerodynamic performance of the wings. Figure (3.17) shows that compared to the clean airfoil, the lifting force decreased by $2.64 \%$, the drag force increased by $17.26 \%$, and the lift-to-drag ratio dropped by $16.84 \%$.

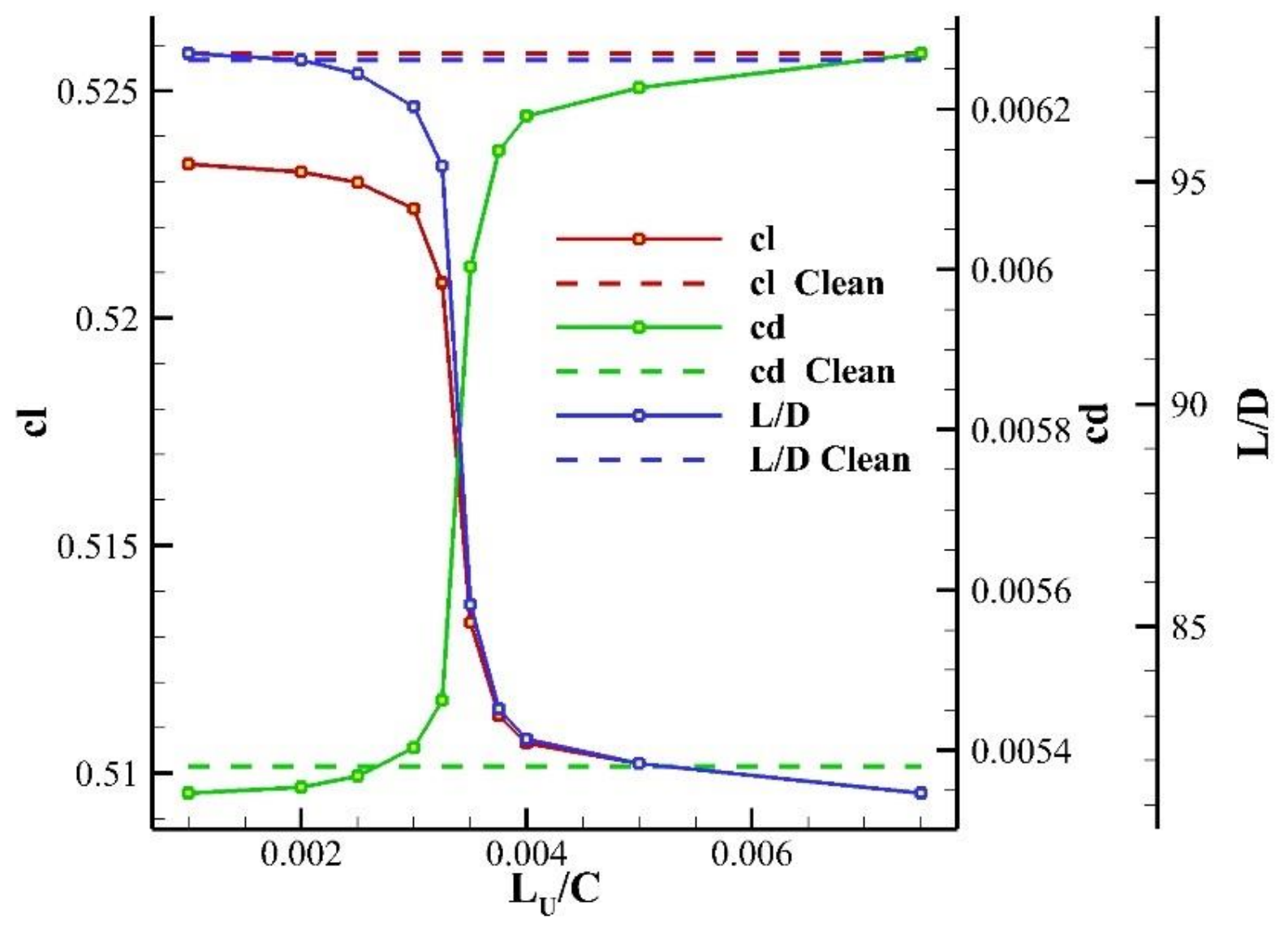

Figure 3.17. Lift coefficient cl, drag coefficient cd and lift-to-drag ratio cl/cd of a NACA 2412 airfoil with trenches located at $\mathrm{X} / \mathrm{C}=0.25$ with different widths.

This degradation in the aerodynamic performance can be mitigated by considering the nondimensional number $L / \theta * \sqrt{R e_{\theta}}$ in the designing process. If the flight conditions are known, the boundary layer momentum thickness and the Reynolds number based on the boundary layer momentum thickness can be calculated or estimated numerically at the location of where the gap will be inserted. Knowing this information and keeping in mind that the non-dimensional number 
$L / \theta * \sqrt{R e_{\theta}}$ must be lower than 600 to maintain the laminar state of the boundary layer, the maximum allowable length of the cavity can be estimated.

Table 3.2. Locations of trenches installed over the NACA 2412 airfoil, the momentum thickness at these locations, the Reynolds momentum thickness, shape factor, and the maximum allowable length of the trench calculated based on the relation $L / \theta * \sqrt{R e_{\theta}} \leq 600$.

\begin{tabular}{ccccc}
\hline \hline $\begin{array}{c}\text { Location of } \\
\text { the gap } \\
\mathbf{X} / \mathbf{C}\end{array}$ & $\boldsymbol{\theta} / \mathbf{C}$ & $\boldsymbol{R} \boldsymbol{e}_{\boldsymbol{\theta}}$ & $\boldsymbol{S}=\frac{\boldsymbol{\delta}^{*}}{\boldsymbol{\theta}}$ & $\begin{array}{c}\text { Allowable } \\
\text { L/C *10 }\end{array}$ \\
\hline & & Upper Surface & \\
\hline 0.1 & $6.8405 \mathrm{e}-5$ & $4.0205 \mathrm{e}+2$ & 2.3549 & 2.0469 \\
\hline 0.2 & $1.0350 \mathrm{e}-4$ & $6.0834 \mathrm{e}+2$ & 2.4733 & 2.5178 \\
\hline 0.3 & $1.3375 \mathrm{e}-4$ & $7.8611 \mathrm{e}+2$ & 2.5496 & 2.8622 \\
\hline 0.4 & $1.6558 \mathrm{e}-4$ & $9.7319 \mathrm{e}+2$ & 2.7416 & 3.1846 \\
\hline 0.5 & $1.9825 \mathrm{e}-4$ & $1.1652 \mathrm{e}+3$ & 2.8235 & 3.4846 \\
\hline & & Lower Surface & & 2.2160 \\
\hline 0.1 & $8.0173 \mathrm{e}-5$ & $4.7121 \mathrm{e}+2$ & 2.5630 & 2.7621 \\
\hline 0.2 & $1.2455 \mathrm{e}-4$ & $7.3209 \mathrm{e}+2$ & 2.6621 & 3.1486 \\
\hline 0.3 & $1.6186 \mathrm{e}-4$ & $9.5134 \mathrm{e}+2$ & 2.8017 & \\
\hline \hline
\end{tabular}

Based on this designing criterion, a numerical test case was conducted where five cavities were installed on the upper surface of a NACA 2412, and three cavities were installed on the lower surface of the airfoil. A clean airfoil numerical case was used to calculate $\theta$ and $R e_{\theta}$ at the leading edge of each cavity location. Based on the condition of laminar state $\frac{L}{\theta} * \sqrt{R e_{\theta}}<600$, the maximum allowable length of the cavity is calculated. Table (3.2) shows the locations of the cavity along the chord-wise direction $X / C$, the momentum thickness $\theta / C$, Reynolds momentum thickness $R e_{\theta}$, shape factor $\mathrm{S}$, and the maximum allowable gap length that will not trigger the transition of the boundary layer calculated based on the condition $\frac{L}{\theta} * \sqrt{R e_{\theta}}<600$.

A numerical test case was created for a NACA 2412 with five gaps on the upper surface and 3 gaps on the lower surface. The dimension of each gap was calculated from the values shown in the last column of table (3.2) after applying a factor of safety of 1.35. The flow Reynolds number is set to 5.7e6, a Mach number of 0.2 and a zero angle of attack. Under these conditions, the eight gaps were located in sections of the airfoil where the boundary layer is still laminar as it can be 
observed from the values of the shape factor $S$ tabulated in table (3.2). Figure (3.18) shows the intermittency contour plot of the flow around the airfoil, with a close-up on the cavities. The intermittency is usually a measure of the irregular alternation of phases. In the SST-transitional turbulence model, the value of intermittency can distinguish between laminar regimes and turbulent ones. When the intermittency value is 0 , the SST turbulence model is suppressed and the flow is treated as a laminar flow, while at a maximum value of 1 the SST model is active and the flow is fully turbulent.

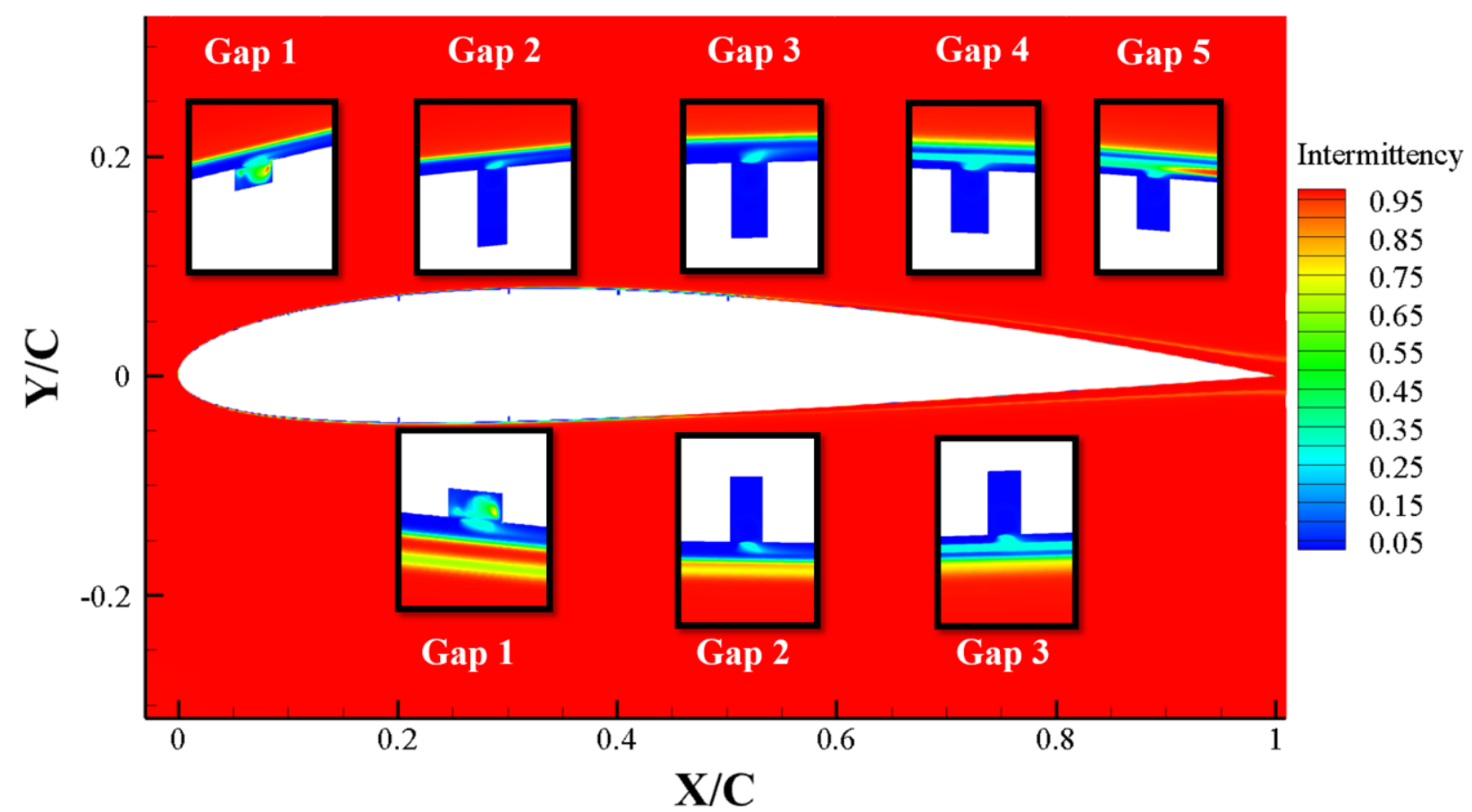

Figure 3.18. Contour plot of the intermittency function over the trenched airfoil. The zoom-in over the cavities show that the boundary layer maintained its laminar state.

It can be observed from figure (3.18) that the boundary layer bypassed the eight cavities, and that the discontinuity did not trigger the transition of the boundary layer. The aerodynamic performance of the airfoil even showed very good agreement with the performance of the clean airfoil. The lift coefficient of the clean airfoil was calculated as 0.249 , and for the trenched airfoil it was found to be 0.25 . The drag coefficient of the clean airfoil was found to be 0.0048 , which is slightly lower than that of the trenched airfoil which was found to be 0.005 . The lift-to-drag ratio is 51 for the clean airfoil, and 49 for the trenched airfoil. The drag coefficient of the trenched airfoil is slightly higher than that of the clean airfoil due to a small upstream shift in the location of the boundary layer transition. The natural transition point of the boundary layer on the upper surface of a clean airfoil is found to be at $0.52 \mathrm{C}$, while in case of the trenched airfoil, it has been shifted to $0.51 \mathrm{C}$. 
Similarly on the lower surface, the boundary layer transition has been shifted from $0.45 \mathrm{C}$ to $0.42 \mathrm{C}$ for the clean airfoil, and the trenched airfoil, respectively.

To emphasize the importance of designing the cavities based on the condition $\frac{L}{\theta} * \sqrt{R e_{\theta}}<600$, another test case was conducted with the same eight gaps on a NACA 2412 airfoil, and the gaps are located at the same locations shown in table (3.2), but this time, the gaps' lengths are fixed at 0.005C. The same boundary conditions used in the test case shown in figure (3.18) are used in this test case. Figure (3.19) shows the intermittency function contour plot over this ill-designed trenched airfoil. It can be observed that the boundary layer experienced transition at the first cavity it encountered on either side of the airfoil.

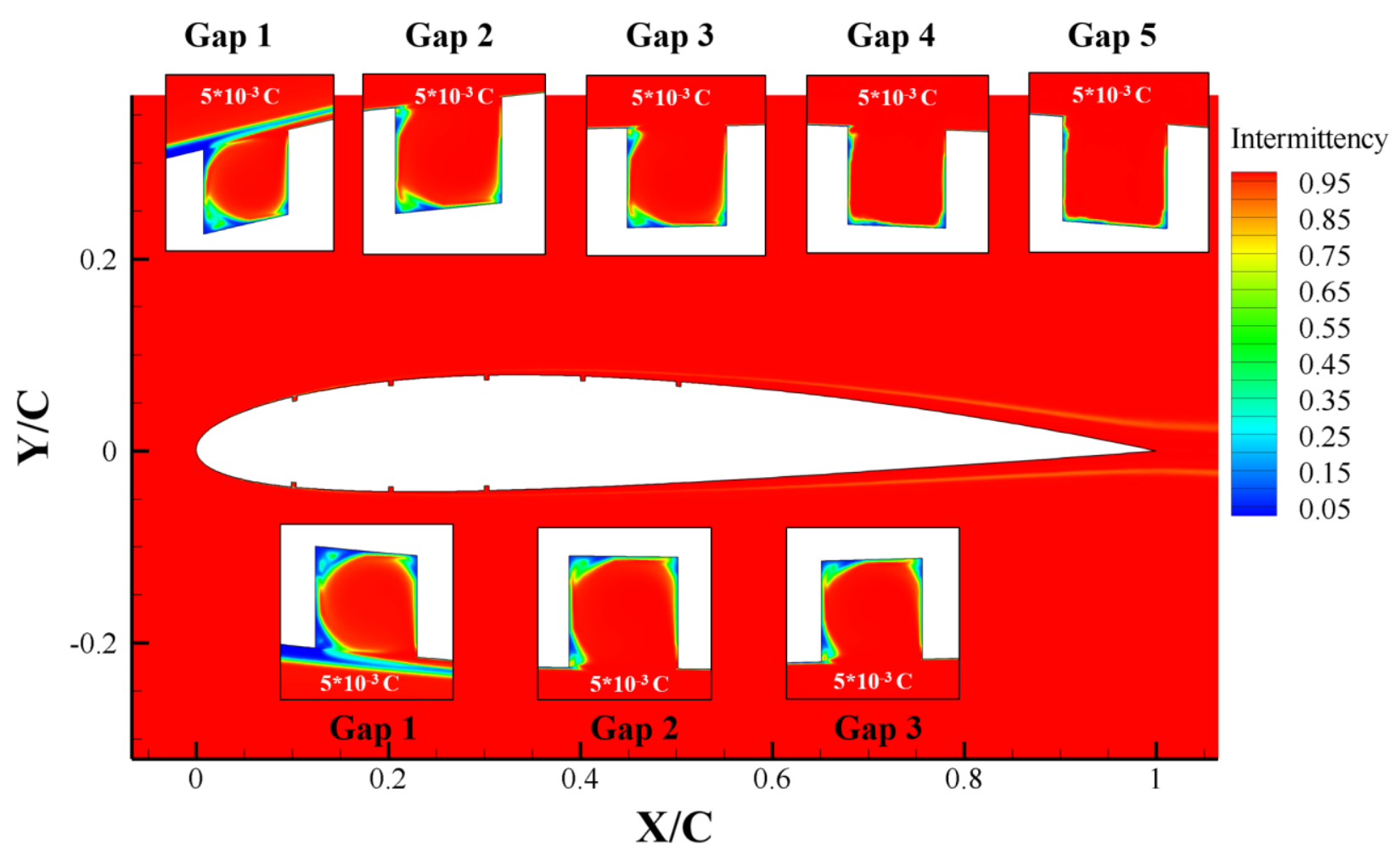

Figure 3.19. Contour plot of the intermittency function over the ill-designed trenched airfoil. The close-up on the cavities show that the boundary layer experienced transition from the first cavity.

The lift coefficient of this ill-designed trenched airfoil was found to be 0.229 , while in case of a well-designed trenched airfoil that used the formula $\frac{L}{\theta} * \sqrt{R e_{\theta}}<600$, the lift coefficient was 0.25 . This represents an $8.4 \%$ drop in the lifting capabilities of the airfoil. The drag coefficient increased by $69.2 \%$ compared to the well designed trenched airfoil, with the values increasing from 0.005 to 0.00846 . This is equivalent to a $44.7 \%$ drop in the lift-to-drag ratio. This second case emphasizes 
the importance of keeping the non-dimensional number $L / \theta * \sqrt{R e_{\theta}}$ blow 600 when introducing cavities or trenches on surfaces with laminar upstream boundary layers.

Unfortunately, it is not always the case that the panels are separated by gaps or cavities. Figure (1.5) showed that in some morphed configurations, the panels have a combination of gaps and backward-facing steps. For that reason, chapter 4 is dedicated to studying the aerodynamics of airfoils when a backward-facing step is installed on either sides. 


\section{AERODYNAMICS OF OVERLAPPING SLIDING SKIN WITH BACKWARD-FACING STEPS}

\subsection{Introduction:}

Overlapping sliding skin is one of the potential morphing designs that fulfills the mechanical requirements of morphing skins. From the aerodynamic perspective, the overlap between two panels is regarded as a backward-facing step employed along the chord length of the airfoil. This discontinuity results in a separation of the flow at the step edge and induces a low-pressure recirculation zone at the step vicinity which alters the aerodynamics of the whole airfoil. Figure (4.1) shows the streamlines of the flow and the recirculation zone aft the backward-facing step.

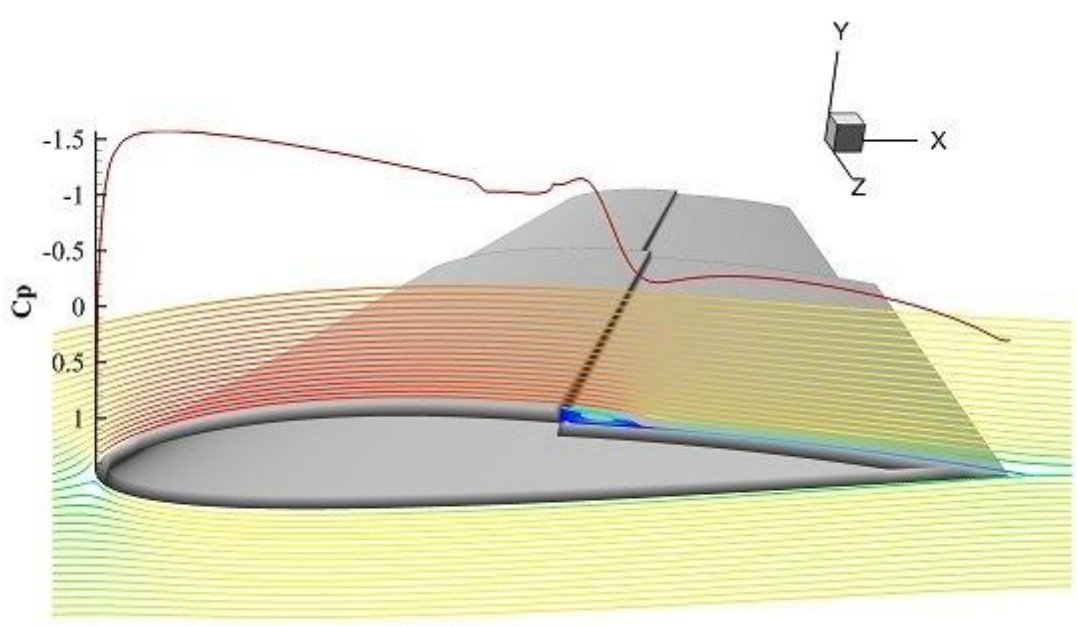

Figure 4.1. Streamlines and pressure coefficient distribution over an overlapping morphing $\operatorname{skin}\left(\operatorname{Re}=5.7 \mathrm{e} 6, \mathrm{M}=0.2\right.$, and $\left.\alpha=2.5^{\circ}\right)$. 
The backward-facing step was first introduced to the aviation community in the 1960s when Richarde Kline and his colleague Floyd Fogleman designed a paper airplane that can fly longer distances despite wind and turbulence. The paper airplane was designed with wings that are flat on the upper surface and partially hollowed at the bottom surface. In 1972, the two men filled a U.S. patent [80] for their wedged-like airfoil whose lower surface is hollowed. Two years later, the National Aeronautics and Space Administration (NASA) sponsored and conducted an experimental study [81] to examine the Kline-Fogleman patented designs. The results of these experimental tests showed that the new airfoil offered no advantages over conventional airfoils, and that the lift-to-drag ratio of the newly patented airfoil is lower than that of a flat plate. Despite these claims, the Kline-Fogleman airfoils were the source of inspiration for Fertis and Smith [82] who designed an airfoil with a backward-facing step, unlike the original design of Kline and Fogleman, Fertis and Smith introduced the backward-facing step on the suction side of the wing. They filled a U.S. patent for their design titled 'Airfoil'.

Fertis published the experimental results of their design six years later [82]. The wind tunnel tests involved a NACA 23012 airfoil with a backward-facing step on its upper surface over a range of Reynolds numbers from $1 \times 10^{5}$ to $5.5 \times 10^{5}$ and different angles of attack. Results showed improved stall characteristics at all tested airspeeds, increased lift coefficients and increased liftto-drag ratios over a wide range of angles of attack. The enhanced performance observed by Fertis [82] was not in perfect agreement with the results obtained by the numerical and experimental testing done by Finaish and Witherspoon [83]. In this study [83], they followed a more systematic way of examining 15 different configurations of a symmetric NACA 0012 airfoil. Backwardfacing steps were located on either side of the airfoil, and the Reynolds number used in this study was $5 \times 10^{5}$. Results showed that installing a backward-facing step on the upper surface of the NACA 0012 has decreased the lift-to-drag ratio due to the noticeable increase in the drag, which was directly proportional to the step depth.

In this chapter, a backward-facing step on either side of an airfoil will be numerically studied to investigate the effect of the step location, depth and angle on the airfoil's aerodynamic properties. The chord length of the airfoil used in this study was set to unity, its surface is an adiabatic no-slip wall. The fluid surrounding the airfoil is modeled as an ideal compressible gas with reference temperature of $300 \mathrm{~K}$. The meshing topology used for this analysis is a C-mesh topology that is generated around the airfoil with an extended far-field boundary that is 32 chords away from the airfoil. The far-field boundary conditions are set to pressure far-field with 0.2 Mach number and a Reynolds number of $5.7 \times 10^{6}$. The angle of attack of the flow was set to $2.5^{\circ}$, unless otherwise stated. 


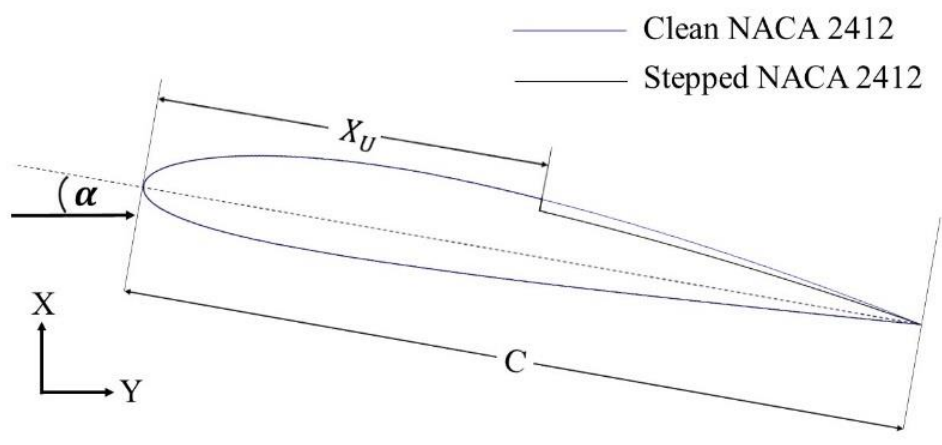

Figure 4.2. A schematic diagram of a clean NACA 2412 (National Advisory Committee for Aeronautics) overlaying the stepped NACA 2412 airfoil.

The baseline design of the stepped airfoil is a NACA 2412 airfoil with a vertically oriented step installed on either side of the airfoil. After the step, the airfoil curvature follows the original NACA 2412 profile, but scaled along the $Y$-direction to create the required descent that matches the step depth. Figure (4.2) shows a schematic diagram of a clean NACA 2412 overlaying an airfoil with a backward-facing step installed on the upper surface of the airfoil and located at a distance $X_{U}$ from the leading edge. This rest of this chapter is divided into two sections, one for the aerodynamic analysis of a step on the upper surface and the other for a step installed on the lower surface of the airfoil. It should be noted here that these installed steps are much larger in size compared to the discontinuities observed on the wings of conventional aircraft. 


\subsection{Aerodynamics of Backward-Facing Steps on the Upper Surface of an Airfoil:}

The first step in any CFD analysis is the challenging task of choosing the optimal density of the mesh to discretize the computational domain. The mesh has to be fine enough to resolve all essential flow features, and at the same time, be computationally affordable in terms of the computational power. To reach this demanding balance, the Richardson's extrapolation method explained in chapter 2 is used on a family of three consecutively refined meshes as shown in figure (4.3).
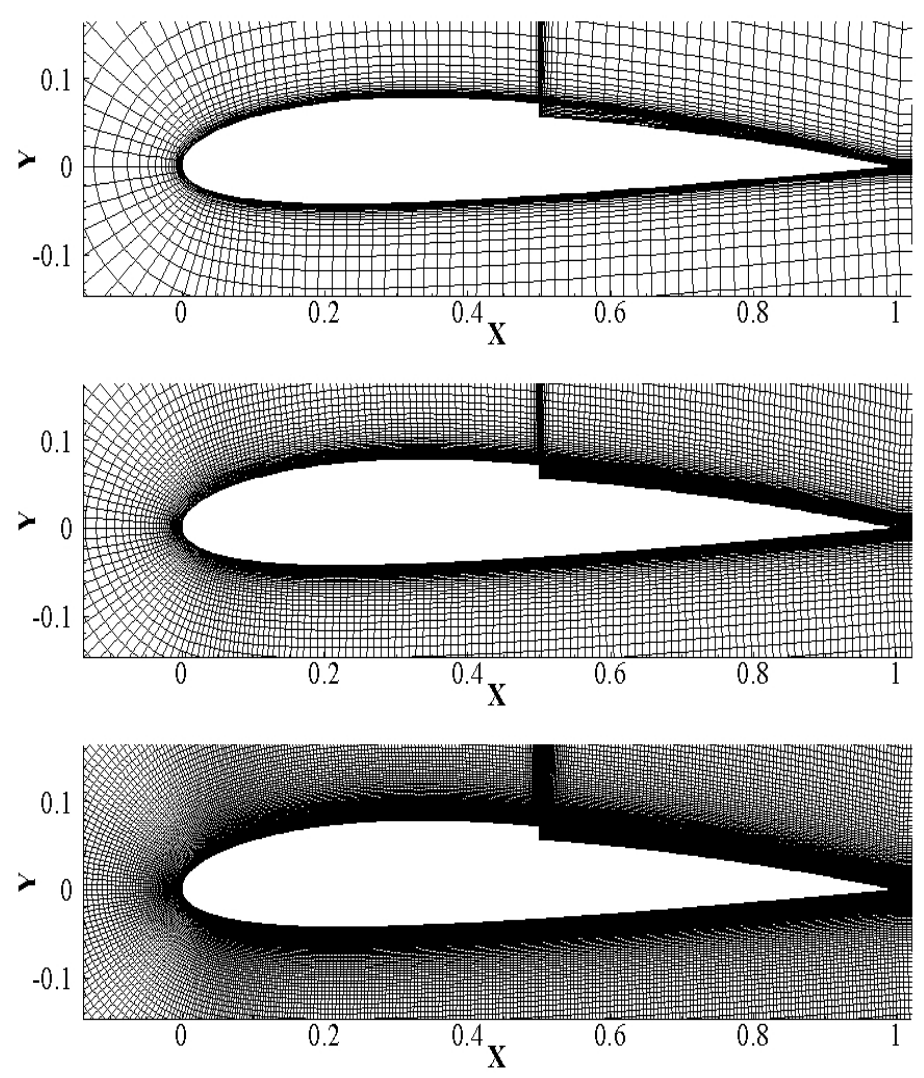

Figure 4.3. Three consecutively refined meshes used to estimate the spatial convergence.

The number of quadrilateral cells in each mesh is approximately 12,000 for the coarse mesh, 48,000 for the medium mesh and 192,000 elements for the fine mesh. The aerodynamic property $\mathcal{F}$ is chosen to be the lift coefficient $c_{l}$. The convergence of the solution is judged by the complete stability of the lift, drag and moment coefficients. Equations (2.1-2.3) were used to assess the 
spatial convergence of the grids used. The order of accuracy of the solution was calculated using equation (2.2) and is shown as the slope of the red line in figure (4.4 a).

The computed order of accuracy $p$ was found to be 2.03 which is in very good agreement with the second order discretization schemes used. Figure (4.4 a) shows that the line joining $\varepsilon_{c}, \varepsilon_{m}$ and $\varepsilon_{f}$ is perfectly parallel to the second order slope line. Figure (4.4 b) shows that the value of the lift coefficient obtained from the fine mesh is $0.244 \%$ away from the asymptotic value $\mathcal{F}_{h=0}$.

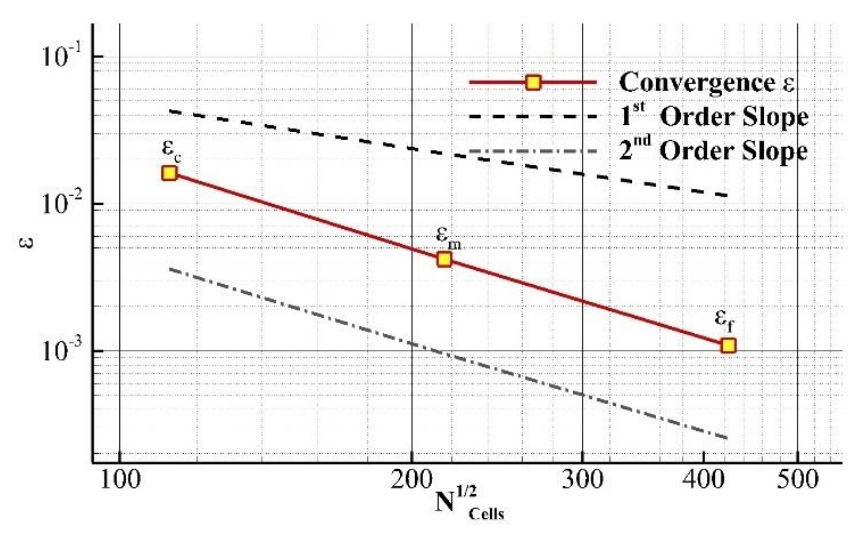

(a)

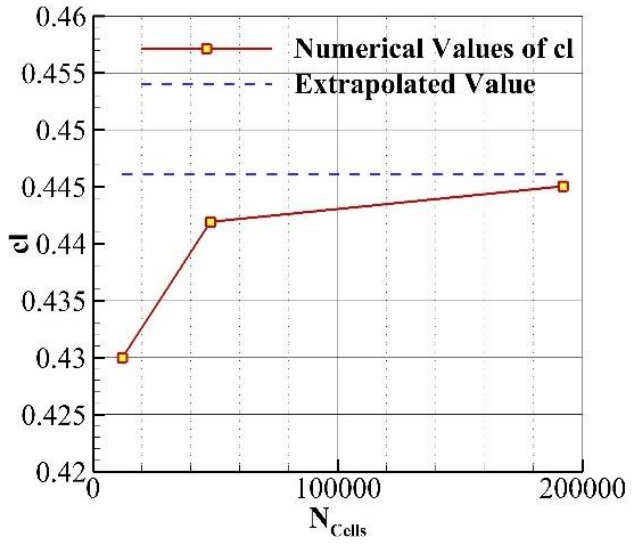

(b)

Figure 4.4. Assessing the mesh spatial convergence using (a) the Richardson's extrapolation method, and (b) the logarithmic slope of the converged errors.

This means that the results obtained from the fine mesh is very close to the asymptotic continuum value $\mathcal{F}_{h=0}$, and can be used to capture the important flow features. Therefore, the fine mesh will be used in this study to examine the aerodynamic performance of the stepped NACA 2412 airfoil. 


\subsubsection{Effect of the step location $X_{U} / C$ :}

The step location on the upper surface of the airfoil is changed from $25 \%$ to $75 \%$ along the unity chord length airfoil, with increments of $5 \%$ from one configuration to the other. This makes a total of 11 configurations that are used to investigate the effect of the step location. Figure (4.5) shows the two extreme positions of the step at $X_{U}=0.25 C$ and $0.75 C$, respectively.

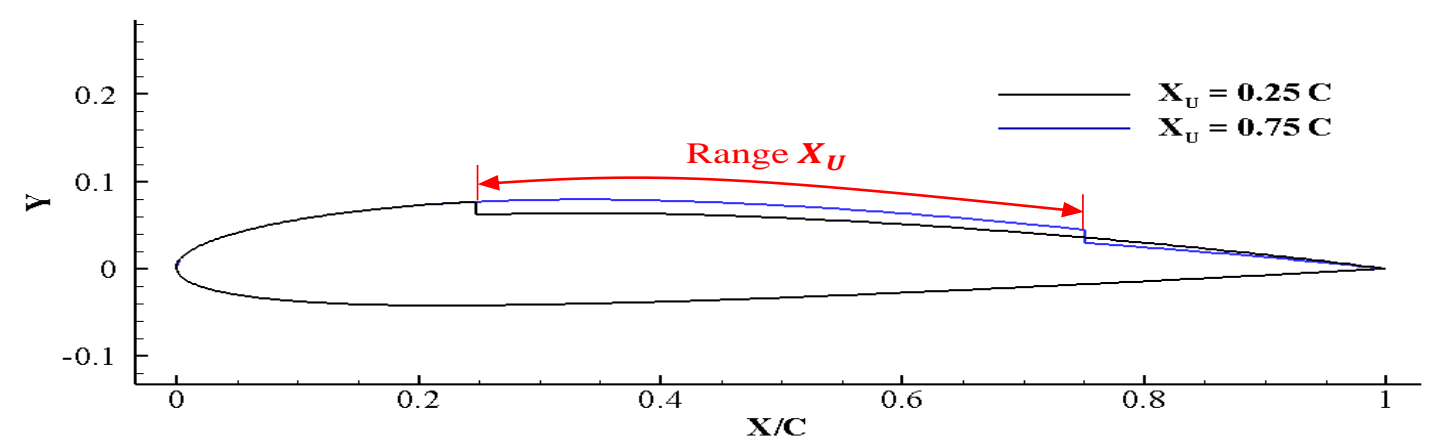

Figure 4.5. The two extreme locations of the step on the upper surface of the NACA 2412.

\subsubsection{Effect of the Step Location on the Lift Coefficient $c_{l}$}

The variation of the lift coefficient $c_{l}$ with the step locations was calculated and plotted as shown in figure (4.6). Two main observations are found in the trend of the curve shown, the first is the direct proportionality relationship between the step location $X_{U}$ and its corresponding lifting force, and the second observation is the sudden change in the slope of the points located before $X_{U} / C=$ 0.4 and the points after $X_{U} / C=0.5$. 


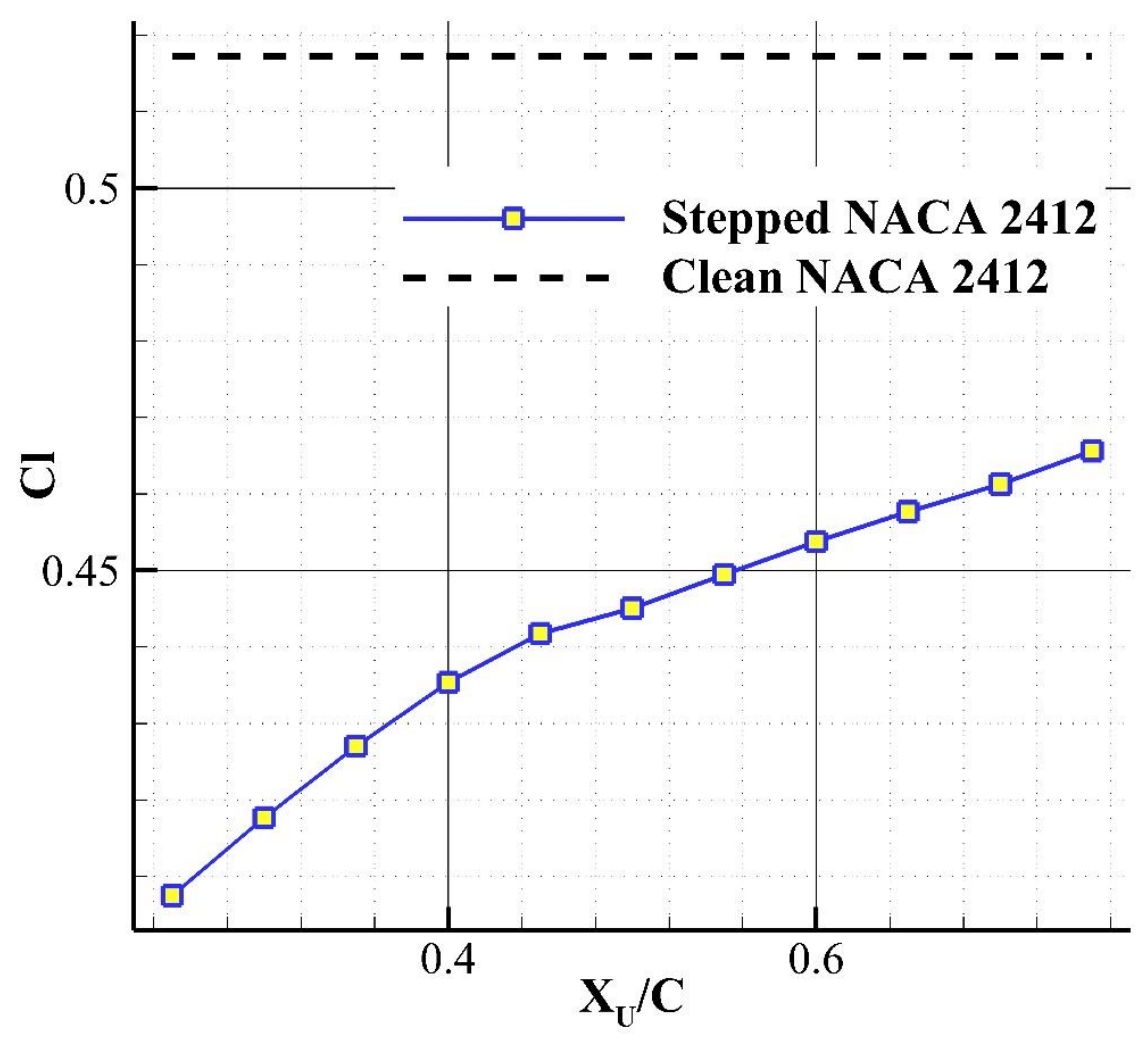

Figure 4.6. The variation of the lift coefficient at different step locations $(\operatorname{Re}=5.7 \mathrm{e} 6, \mathrm{M}=0.2$, and $\alpha=2.5^{\circ}$ ). The dashed red lines emphasize the change in the slope of the obtained values.

The fundamentals of airfoil aerodynamics dictate that the lifting force is mainly influenced by the pressure distribution around the airfoil. Therefore, to investigate the direct relation between the step location and the lift coefficient $c_{l}$, the distribution of the pressure coefficient $C_{p}$ over the stepped airfoil is plotted in figure (4.7) for cases with different step locations, as well as the pressure distribution over a clean NACA 2412 airfoil. 


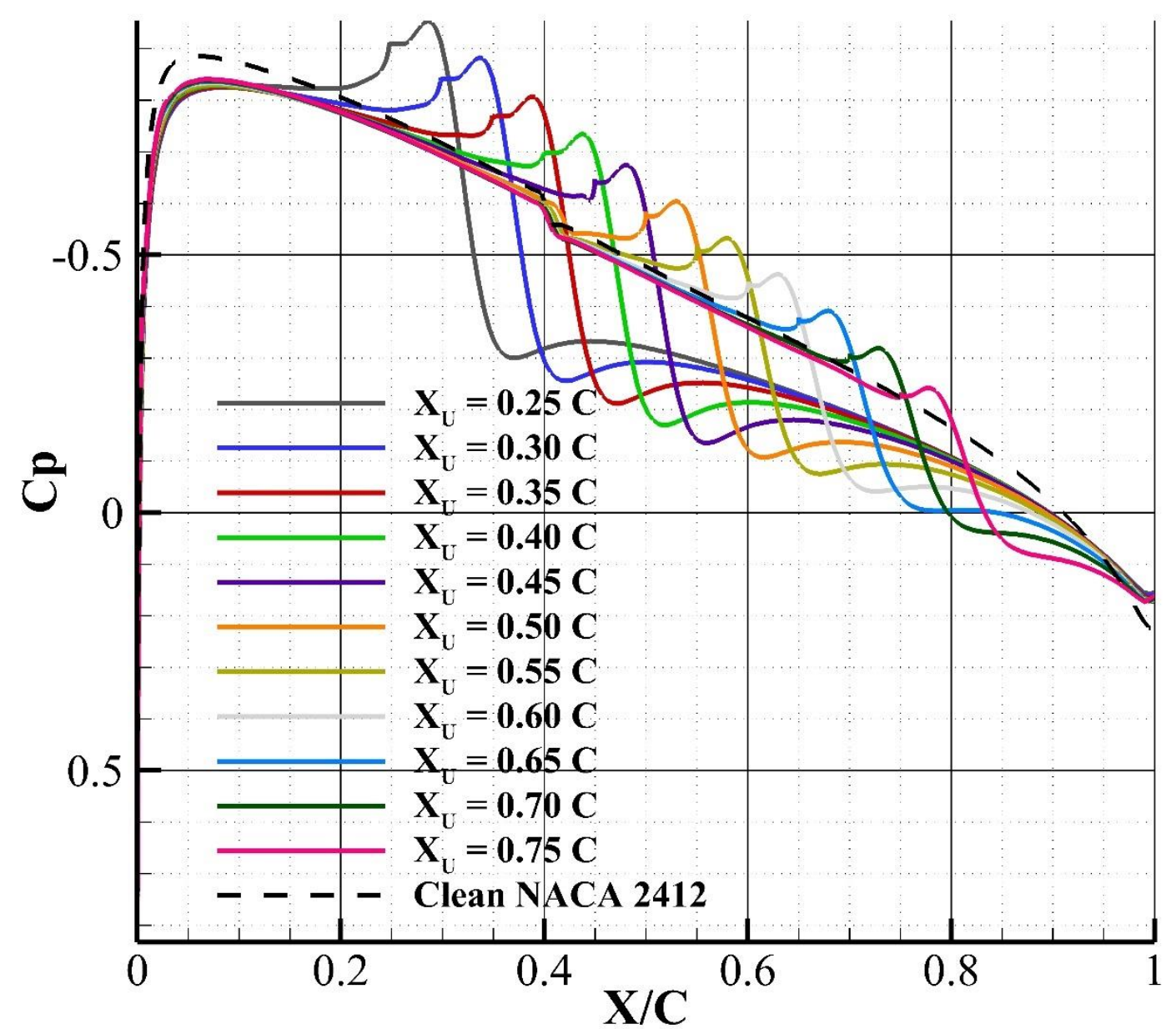

Figure 4.7. The pressure distribution over the upper surface of stepped airfoils with different step locations as well as the pressure distribution over the clean NACA 2412 airfoil $(\mathrm{Re}=5.7 \mathrm{e} 6$, $\mathrm{M}=0.2$, and $\alpha=2.5^{\circ}$ ).

With the spatial acceleration of the flow over the airfoil's leading-edge curvature, the pressure field experienced a drop in its value, and gradually starts to recover, when suddenly the flow separates from the airfoil surface at the backward-facing step leading edge. The separated flow creates a low-pressure recirculation zone at the step vicinity, hence the drop in the pressure distribution on the upper surface of the airfoil. However, the sudden reduction in the airfoil thickness after the step, relatively decreases the flow velocity which results in a subsequent highpressure region that extends to the trailing edge of the airfoil. Figure (4.8) shows the separation of the flow and its subsequent reattachment at the end of the recirculation zone. It should be noted 
here that the numerical solution managed to capture three different length scales of vortices trapped at the corner of the step. Theoretically, a large number of closed eddies with decreasing sizes and strengths is expected.

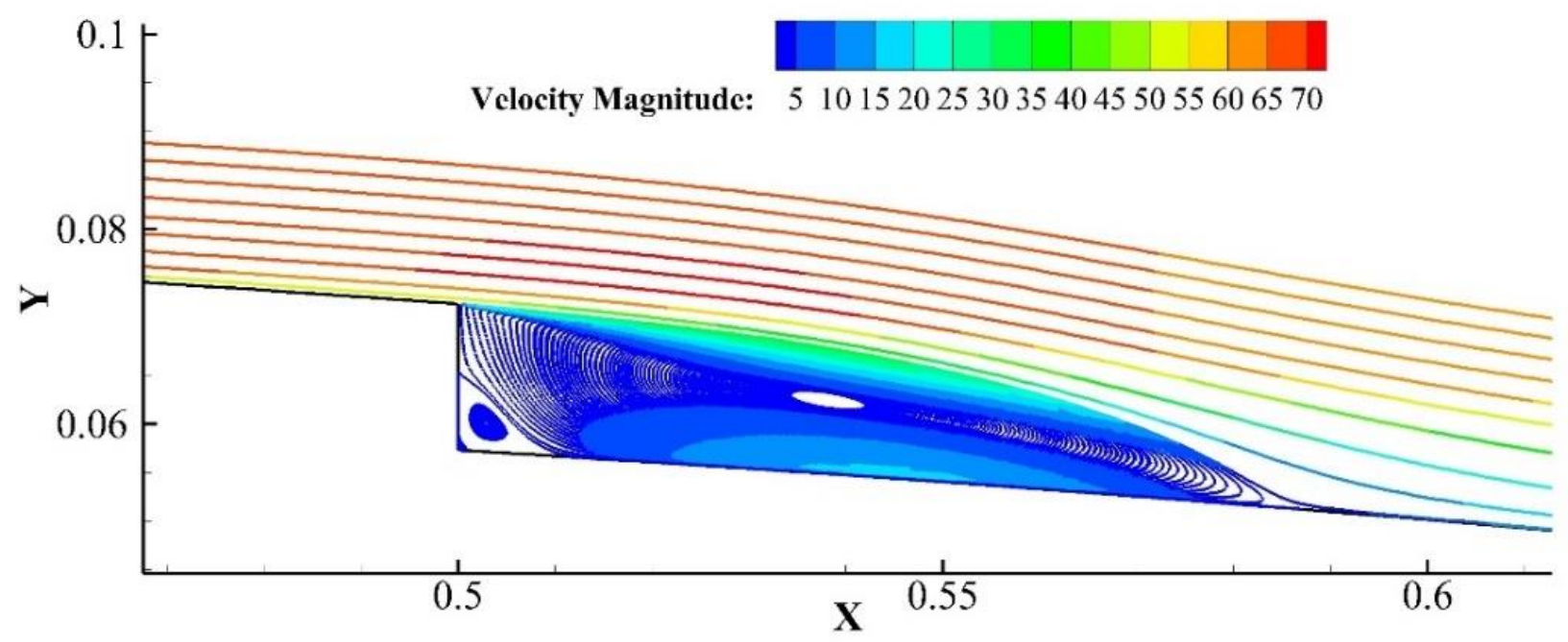

Figure 4.8. Streamlines showing the separation of the flow at the step edge and the creation of low pressure recirculation zones over the step's vicinity. The step is located at the mid-chord length on the suction side of a NACA $2412\left(\operatorname{Re}=5.7 \mathrm{e} 6, \mathrm{M}=0.2\right.$, and $\left.\alpha=2.5^{\circ}\right)$.

The change in the airfoil thickness, and consequently the increase of the pressure distribution over the suction side of the airfoil significantly affects the lifting capabilities of the airfoil and drops it below the value obtained from the clean NACA 2412 airfoil. At the same boundary conditions, a clean NACA 2412 obtains a lift coefficient value of 0.5172 which exceeds all the values obtained from the stepped airfoil configurations as shown in figure (4.6). As the step location shifts towards the trailing edge, the region of high pressure diminishes, and the lift coefficient increases. This justifies the direct proportionality relationship between the step location and the value of lift coefficient $c_{l}$.

The second observation shown in figure (4.6) is the change of the slope of the lift coefficient curve for the points located before $X_{U} / C=0.4$ and the points after $X_{U} / C=0.5$. This change in the slope is mainly driven by the relative position of the step with respect to the point at which the laminar boundary layer experiences a transition from laminar to turbulent. At the chosen boundary conditions, the natural transition of the boundary layer from laminar to turbulent is approximately at $X / C=0.425$. For cases with a step located before this natural transition point, the presence of 
the step triggers the transition before the point of natural transition, prolonging the instability of the boundary layer.

\subsubsection{Effect of the Step Location on the Drag Coefficient $c_{d}$}

In each of the eleven configurations, the drag coefficient was calculated, and the relation between drag coefficient and the location of the step is shown in figure (4.9).

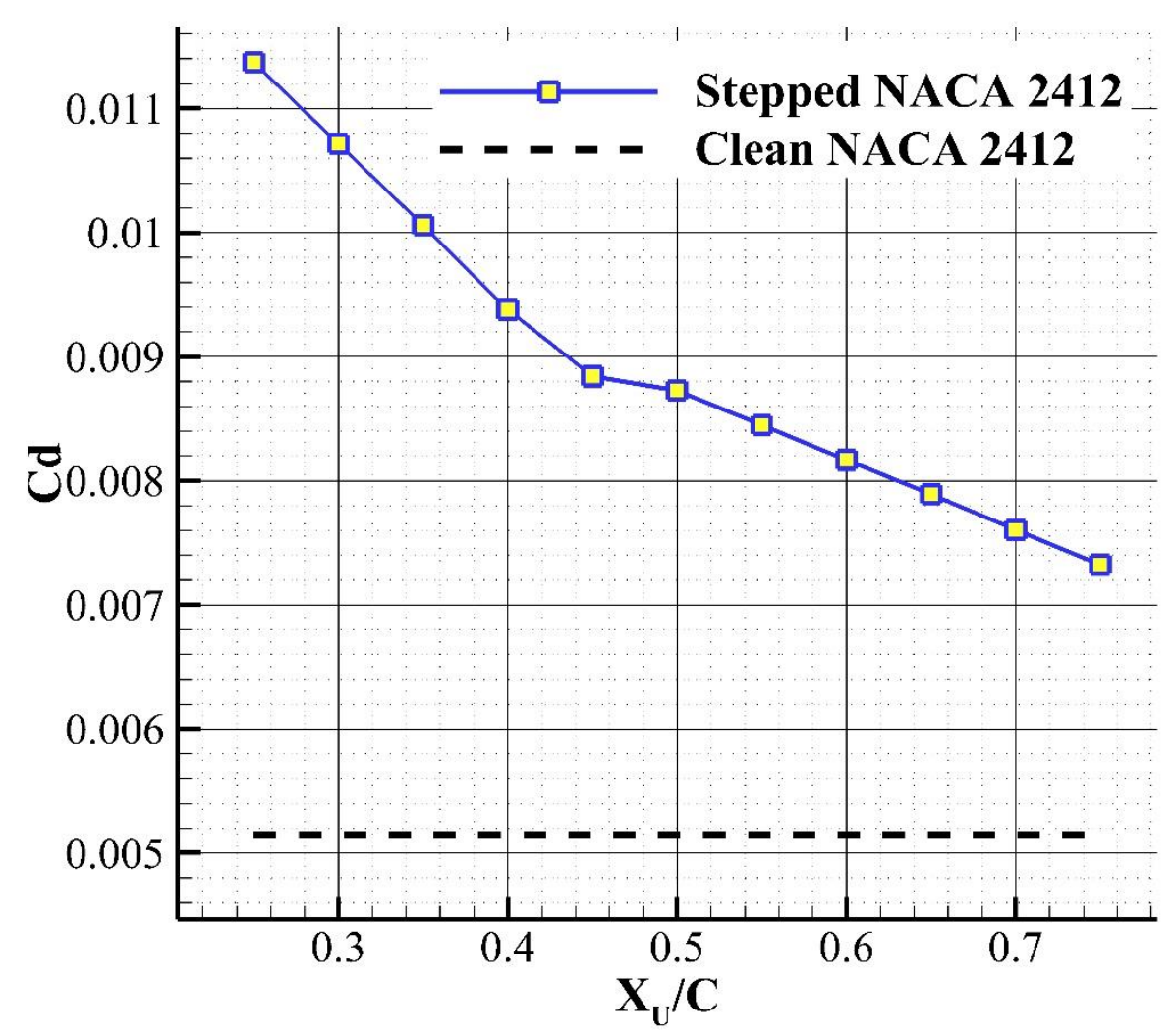

Figure 4.9. The variation of the drag coefficient at different step locations $(\operatorname{Re}=5.7 \mathrm{e} 6, \mathrm{M}=0.2$, and $\alpha=2.5^{\circ}$ ).

Similar to the observations in figure (4.6), figure (4.9) reflects two main characteristics in the relationship between the drag coefficient $c_{d}$ and the step location. The first observation is that the drag coefficient has an inverse relationship with the step location. As the step location shifts from the leading edge of the airfoil towards the trailing edge, the drag coefficient value decreases. The second observation is again related to the sudden change in the slope of the lines before and after 
the location of transition of the viscous boundary layer, which is approximately at $X / C=0.425$. For streamlined bodies, viscous drag dominates the pressure drag, as it is the case of airfoils with small angles of attack. This makes the graphs of the wall shear stresses $\tau_{w}$ or the skin friction coefficient, a good tool to compare the drag forces on different airfoils designs. Equation (4.1) shows that as the area under the curves of the skin friction coefficient increases, the viscous forces and consequently the total drag forces increase. Therefore, to justify the relation between the drag coefficient and the step location, the skin friction coefficient of all cases is plotted in figure (4.10). Figure (4.10) presents the skin friction coefficient distribution in two separate subfigures, because of the different nature of the skin friction coefficient values for cases with a step located before the transition point $(X / C=0.425)$ and after this point. Figure $(4.10$ a) shows the skin friction coefficient distribution in cases when the step is located before the natural transition point. The figure shows that the skin friction coefficient drops smoothly at the laminar section of the viscous boundary layer, but as the flow reaches the edge of the step, the laminar nature of the boundary layer is interrupted and transition from laminar to turbulent takes place.

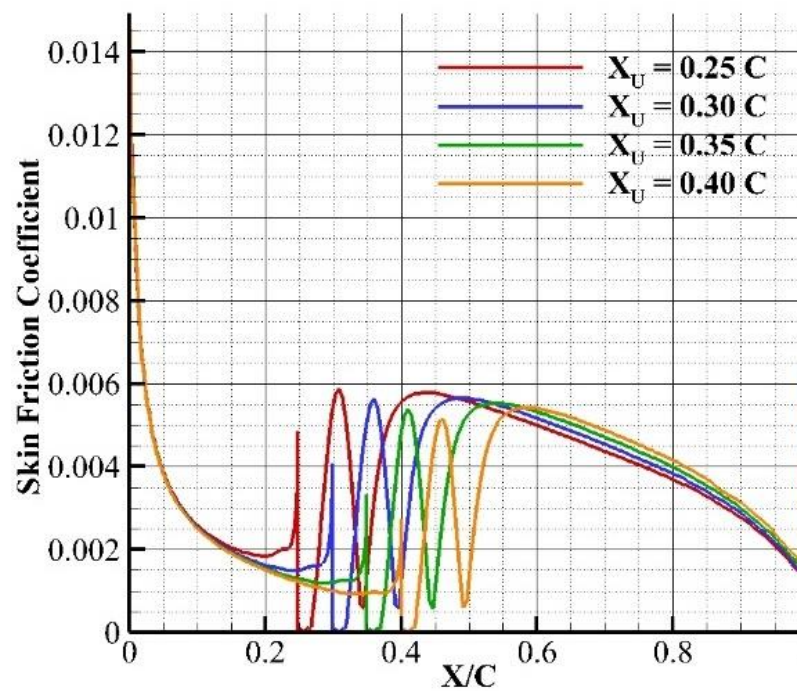

(a)

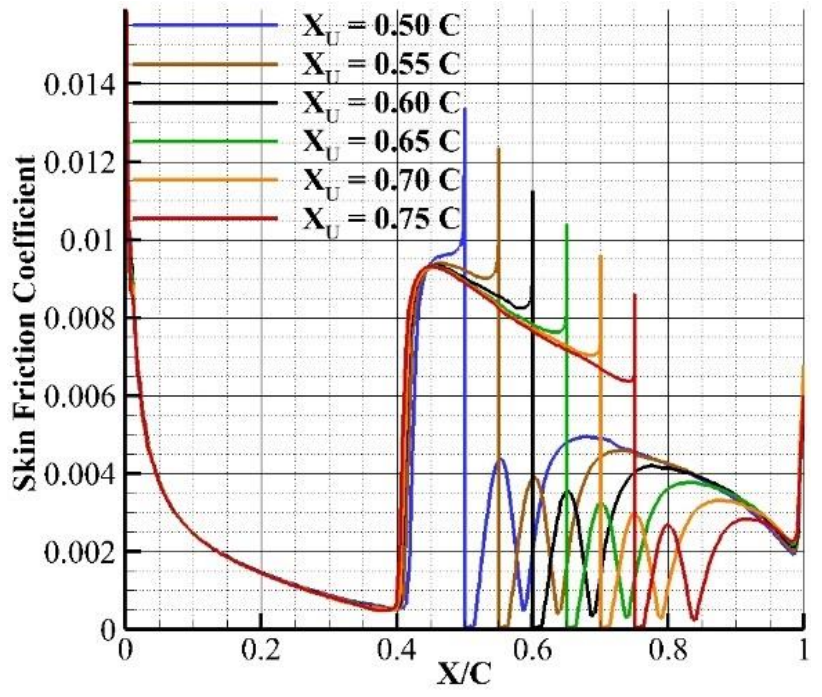

(b)

Figure 4.10. Skin friction coefficient values for airfoils with a step (a) before the viscous boundary layer transition point and (b) after the transition point $(\operatorname{Re}=5.7 \mathrm{e} 6, \mathrm{M}=0.2$, and $\alpha=$ $\left.2.5^{\circ}\right)$.

Right after the step, the skin friction coefficient value drops close to zero due to the presence of low energy eddies trapped at the corner of the step. This is followed by a concaved down curve 
confining the recirculation zone. After the reattachment of the boundary layer at the end of the recirculation zone, the skin friction coefficient follows the natural pattern of a turbulent boundary layer. The curves in figure $(4.10 \mathrm{~b})$ show that the presence of the step after the natural transition point has an important influence on the behavior of the skin friction coefficient. It prolonged the transition region and minimized the turbulent one before dropping towards zero at the tip of the airfoil.

As the step is shifted towards the trailing edge, the area under the curves in figure $(4.10 \mathrm{~b})$ increases, so the drag coefficient should have increased, but this contradicts the values shown in figure (4.9) where the drag coefficient values followed a negative slope when plotted versus the step location. This means that the pressure drag is the dominant component in the drag coefficient value in cases of airfoils with a backward-facing step, and only a decomposition of the drag coefficient can resolve this conflict.

The drag coefficient is decomposed into its main components, namely, the pressure drag and the skin friction drag coefficients as:

$$
c_{d}=c_{p}+c_{f}=\frac{1}{\rho v^{2} A} \int_{S}\left(P-P_{0}\right)(\widehat{n} . \hat{\imath}) d A+\frac{1}{\rho v^{2} A} \int_{S} \tau_{w}(\hat{t} . \hat{\imath}) d A
$$

where $C_{p}$ is the pressure drag coefficient, $c_{f}$ is the friction drag coefficient or viscous drag coefficient, $\rho$ is the fluid density, $v$ is the reference velocity, $A$ is the reference area, $p$ is the pressure at the surface $d A, p_{0}$ is the reference pressure, $\hat{n}$ is a unit vector normal to the surface $d A$, $\tau_{w}$ is the wall shear stresses at the surface $d A$, and $\hat{t}$ is a unit vector tangent to the surface $d A$.

Figure (4.11) shows the decomposed values of the drag coefficient in case of a clean NACA 2412 airfoil and in cases when the step is employed at different locations on the upper surface of the airfoil.

In all cases, the value of the viscous drag coefficient has minor changes from one case to the other, and the variation in the pressure drag component constitutes the major change in the total drag force. Unlike streamlined airfoils, the low-pressure recirculation zone acting on the vertical step creates an adverse force on the airfoil that significantly increases the pressure drag. For that reason, it is insufficient to use the skin friction coefficient values as the sole assessment of the variation of the drag coefficient. The pressure distribution over the airfoil will provide a better assessment to compare the values of the drag coefficient at different step locations. 


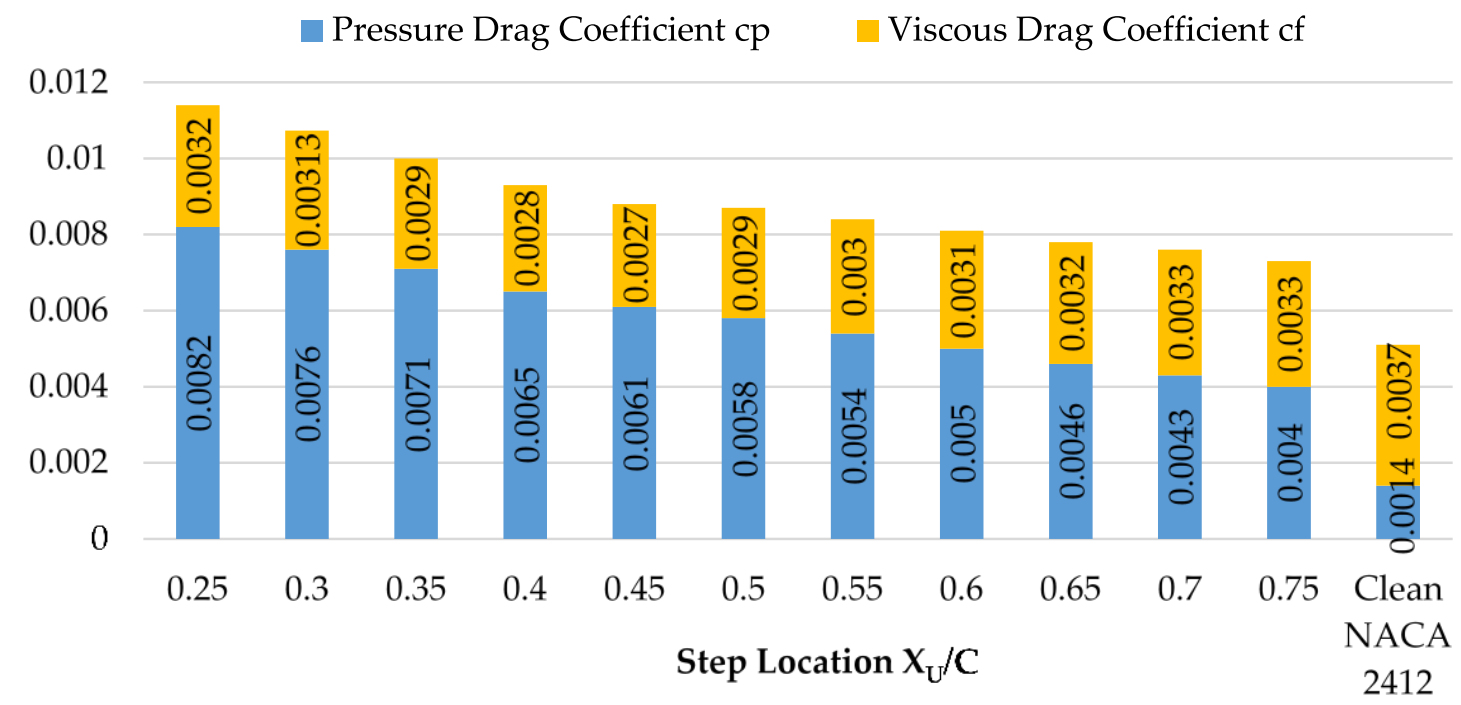

Figure 4.11. Decomposition of the drag coefficient to pressure drag and viscous drag coefficients.

Figure (4.7) showed that a low-pressure zone always exists after each step due to the vortex formation. The range of this minimum pressure varies with the variation of the step location. As the step location increases (moves towards the trailing edge), the pressure after the step relatively increases, so the adverse force acting on the step wall gradually decreases. This explains the inverse relationship between the drag coefficient values and the step location shown in figure (4.9). The change in the slope in figure (4.9) is again attributed to the transition of the viscous boundary layer, where for the cases with the step located before $X / C=0.425$, the presence of the step triggers the transition before the point where it naturally occurs.

\subsubsection{Effect of the Step Location on the Critical Angle of Attack $\alpha_{c r}$}

This part of subsection 4.2.1 tests the effect of the backward-facing step on the critical angle of attack $\alpha_{c r}$ and the near stall behavior of the stepped airfoil. Four different configurations of the NACA 2412 were tested. The first configuration is the clean NACA 2412, without any steps. The second, third and fourth cases incorporated a backward-facing step on the upper surface of the airfoil at $X_{U} / C=0.25,0.5$ and 0.75 , respectively. The four configurations were tested over a wide range of angles of attack $\alpha$ at which the separated flow on the airfoil hinders its ability to create lift. 


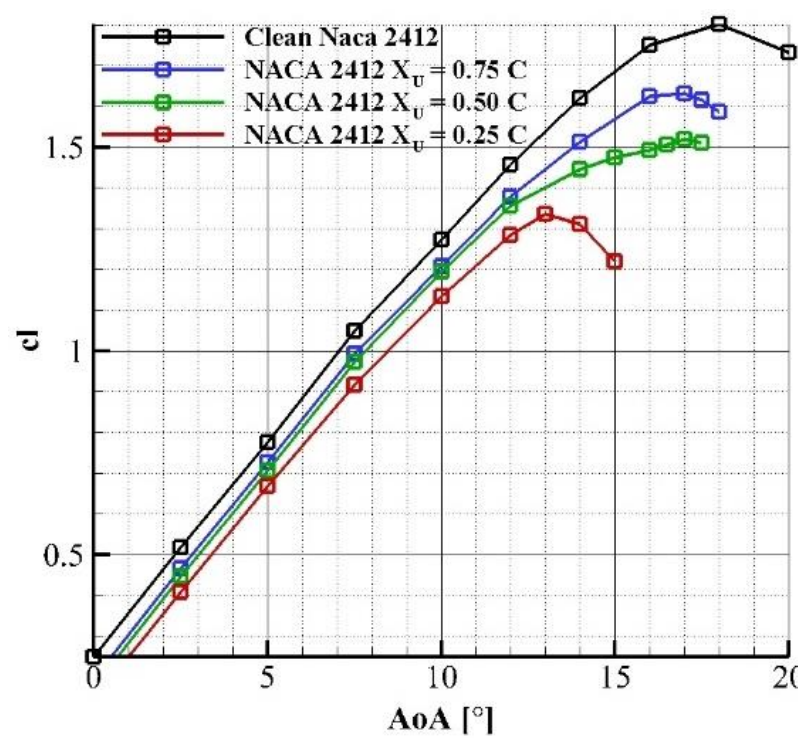

(a)

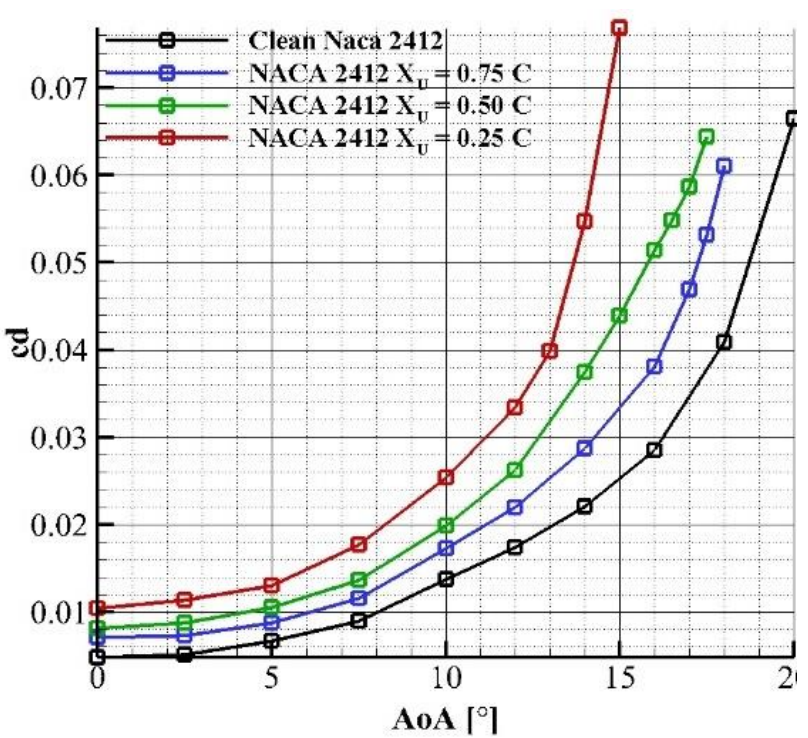

(b)

Figure 4.12. (a) Lift and (b) drag coefficients versus angle of attack $\alpha(\operatorname{Re}=5.7 \mathrm{e} 6$ and $\mathrm{M}=0.2)$.

Figures (4.12 a) and (4.12 b) show the values of the lift coefficient $c_{l}$ and drag coefficient $c_{d}$ at different angles of attack $\alpha$. The lift coefficient values shown in figure (4.12 a) start with a linear relationship that extends up to an angle of attack of $10^{\circ}$. As the separated flow starts to become dominant around the airfoils, the relation between the lift coefficient and angle of attack becomes non-linear and quickly reaches the critical angle of attack $\alpha_{c r}$. It is shown in figure $(4.12 \mathrm{a})$ that the three cases of the stepped airfoil experienced an early onset of stall when compared to the case of the clean airfoil whose $\alpha_{c r}$ is approximately at $18^{\circ}$. While in cases of a backward-facing step at $X_{U} / C=0.25,0.5$ and 0.75 , the critical angle of attack was nearly at $13^{\circ}, 17^{\circ}$ and $17^{\circ}$ respectively. This suggests that installing a backward-facing step on the upper surface of an airfoil speeds up the onset of stall and lowers the critical angle of attack. The drag coefficient values shown in figure (4.12 b) reflect that stepped airfoils will experience higher dragging forces when compared to the clean airfoil. As the step location is shifted towards the leading edge of the airfoil, higher values of the drag coefficient are experienced, and they grow faster with larger angles of attack. These results show that installing a backward-facing step on the upper surface of the NACA 2412 degrades the overall stall behavior of the airfoil. 


\subsubsection{Effect of the step depth $D_{U} / C$}

This subsection will investigate the effect of the step depth on the aerodynamic coefficients of the stepped airfoil. In all the coming numerical cases of this subsection, the step location is fixed at the mid-chord length $(X / C=0.5)$, and the only variable will be the step depth. The first configuration of the stepped airfoil starts with a step depth of $D_{U} / C=0.0075$ and increase to $D_{U} / C=0.025$ with an increment of $0.0025 C$ from one configuration to the other. Figure (4.13) shows the two extreme configurations of the step depths $D_{U} / C=0.0075$ and $D_{U} / C=0.025$, where $D_{U}$ is the step depth and $C$ is the chord length.

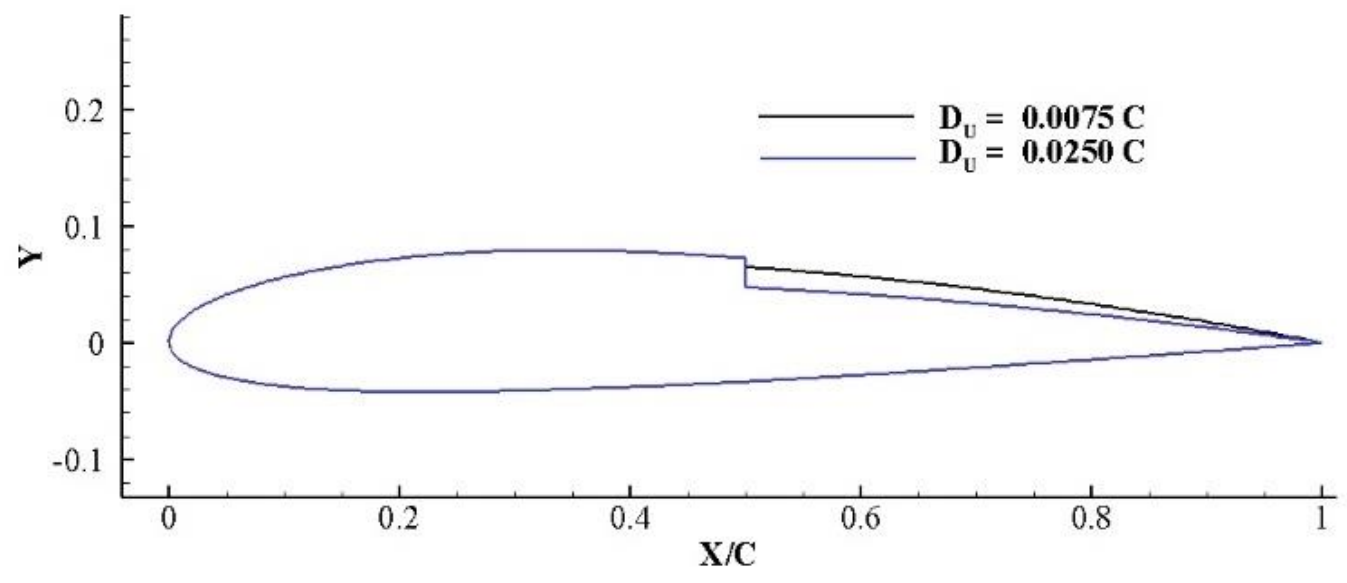

Figure 4.13. The two extreme depths of the step.

As the depth increases from $D_{U} / C=0.0075$ to $D_{U} / C=0.025$ going through the eight different configurations, the lift coefficient decreased by about $20 \%$, and the highest value of lift coefficient obtained from the stepped airfoils $(0.4812)$ is lower than the value obtained by the unchanged (clean) NACA 2412 which is equal to 0.5172 . Figure (4.14) shows the inverse relation between the lift coefficient $c_{l}$ and the step depth $D_{U} / C$. 


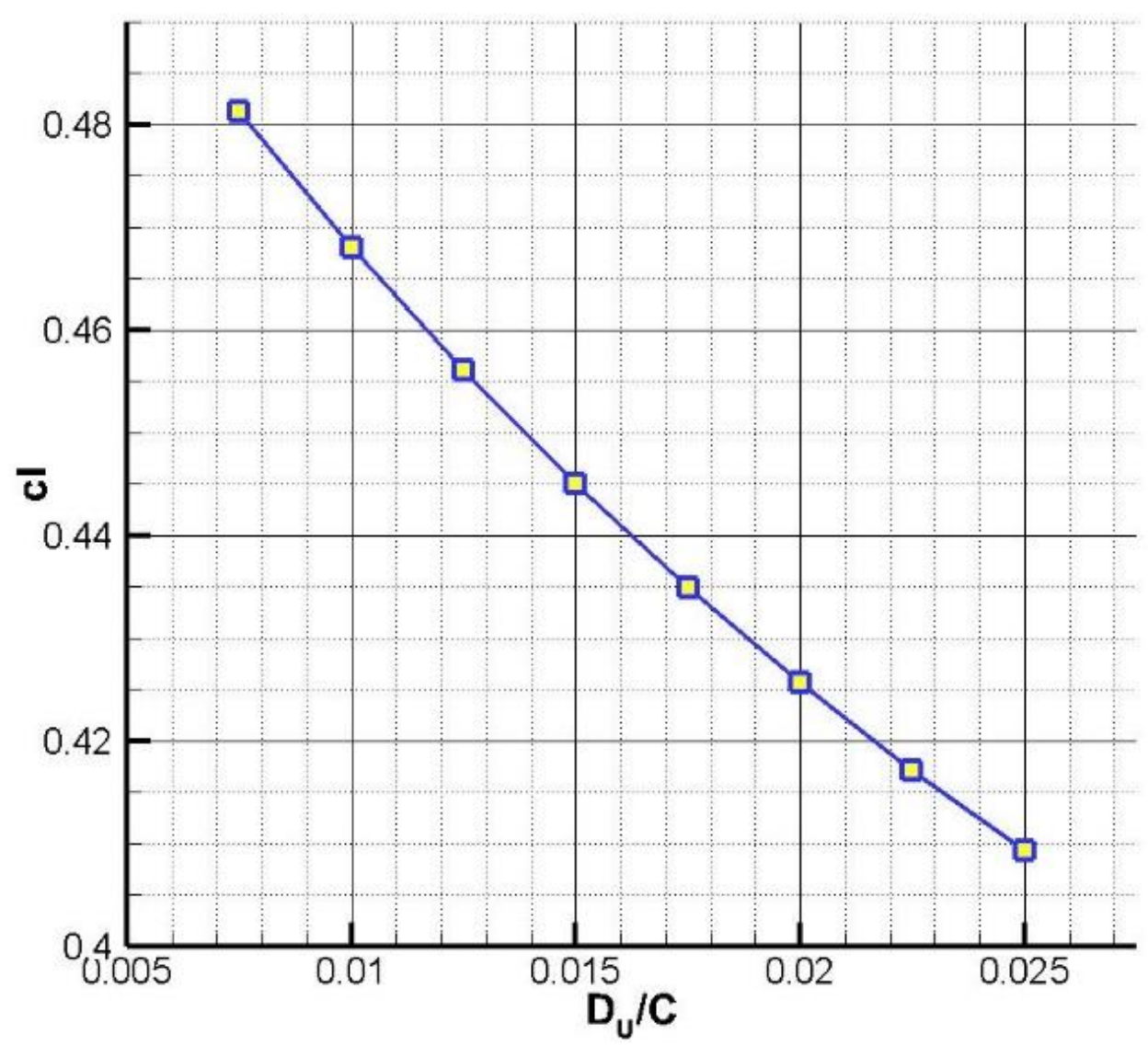

Figure 4.14. Effect of the step depth on the lift coefficient of the stepped NACA 2412 airfoil (Re $=5.7 \mathrm{e} 6, \mathrm{M}=0.2$, and $\alpha=2.5^{\circ}$ ).

The steep slope of the curve shown in figure (4.14) reflects the strong inverse correlation between the step depth and the lifting forces of the stepped airfoil. To study the relationship between the lifting forces and the step depth, the pressure distribution over the eight configurations of step depths is shown in figure (4.15).

The step caused the airfoil thickness to locally decrease, which results in flow spatial deceleration. The conservation of momentum dictates that with the entropy preserved, the pressure will increase after the step location. As the step depth increased, the pressure increased on the suction side leading to an overall drop in the lift coefficient values. 


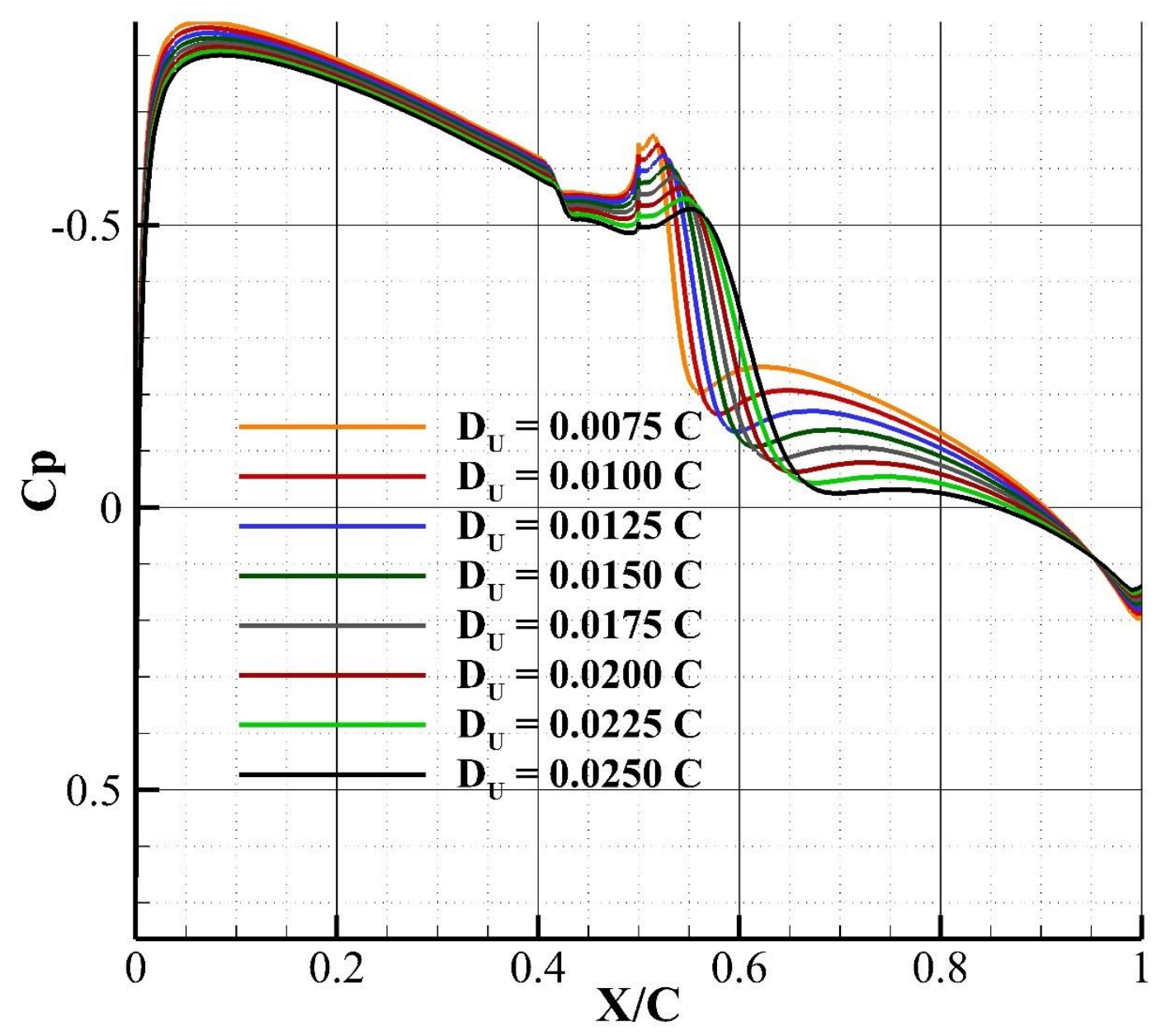

Figure 4.15. Pressure distribution over NACA 2412 airfoils with different step depths $(\mathrm{Re}=$ $5.7 \mathrm{e} 6, \mathrm{M}=0.2$, and $\alpha=2.5^{\circ}$ ).

It was observed that the step does not only affect the pressure distribution after it, but also has a small effect on the pressure distribution before it. It is observed from figure (4.15) that as the step depth increases, the pressure before the step slightly increased too. From this analysis, it could be concluded that an increase in the step depth will cause the lifting forces to decrease.

Similar to the previous analysis, the drag coefficient in each of the eight cases is calculated to establish a relationship between the step depth and the drag acting on the airfoil. Results of these calculations are shown in figure (4.16). 


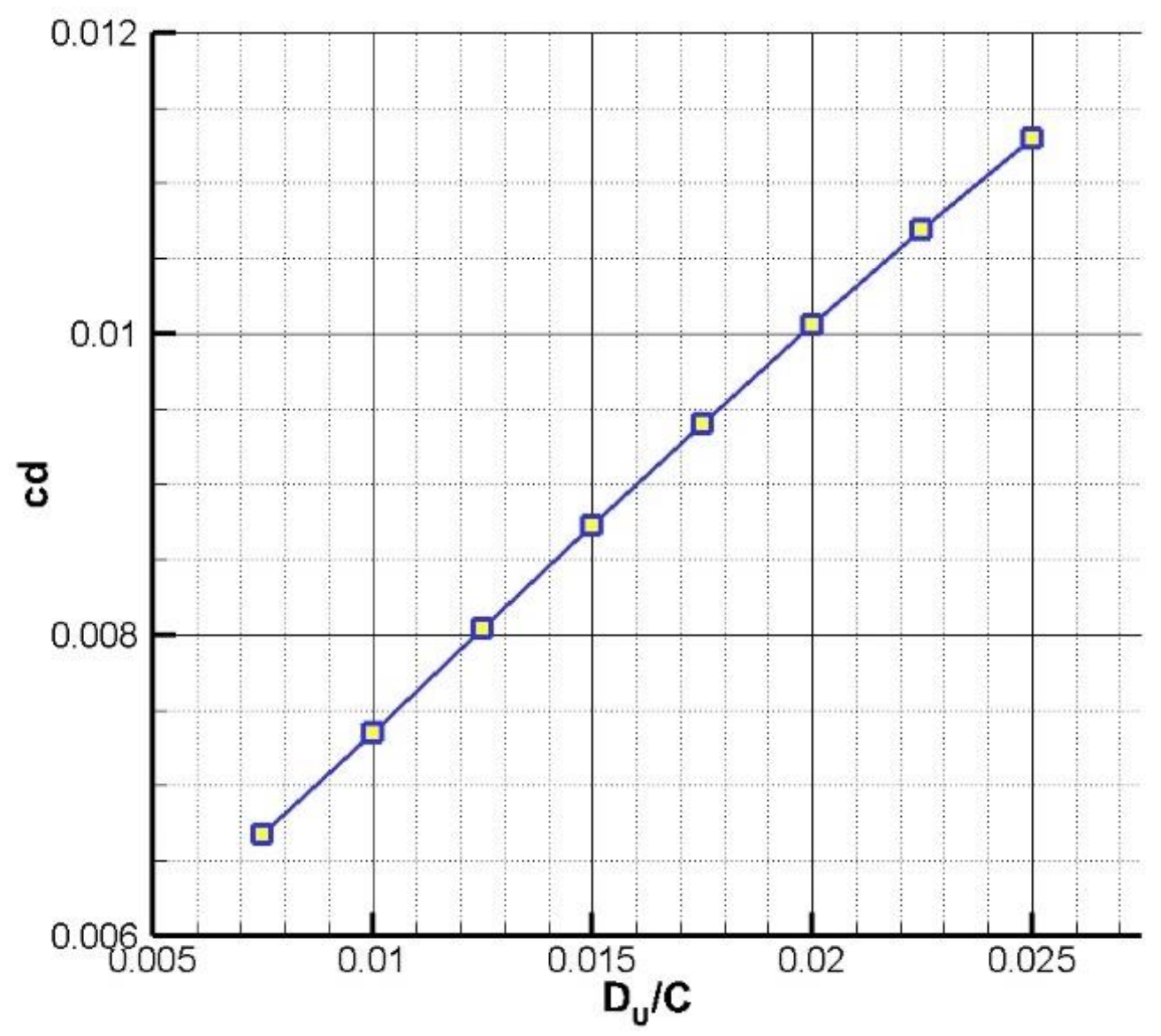

Figure 4.16. Effect of the step depth on the drag coefficient of the stepped NACA 2412 airfoil $\left(\operatorname{Re}=5.7 \mathrm{e} 6, \mathrm{M}=0.2\right.$, and $\left.\alpha=2.5^{\circ}\right)$.

An approximate linear relationship is observed between the depth of the step $D_{U}$ and the drag coefficient of the airfoil. The lowest value of drag coefficients found in the eight configurations is still higher than the value obtained from a clean NACA 2412 at the same conditions where the drag coefficient was found to be equal to 0.00515 . While the lowest value shown in figure (4.16) is observed to be equal to 0.00668 at a step depth of $0.0075 C$.

Figure (4.16) shows that there is a constant increment in the drag value from one case to the other of approximately 7E-4 with each $0.0025 C$ increment in the step depth. This relationship holds over the full testing range. The inverse linear-like relationship shown in figure (4.16) can be better understood by decomposing the drag coefficient into its two main components; the pressure drag coefficient and the viscous drag coefficient using equation (4.1). The results of this decomposition are shown in figure (4.17). 


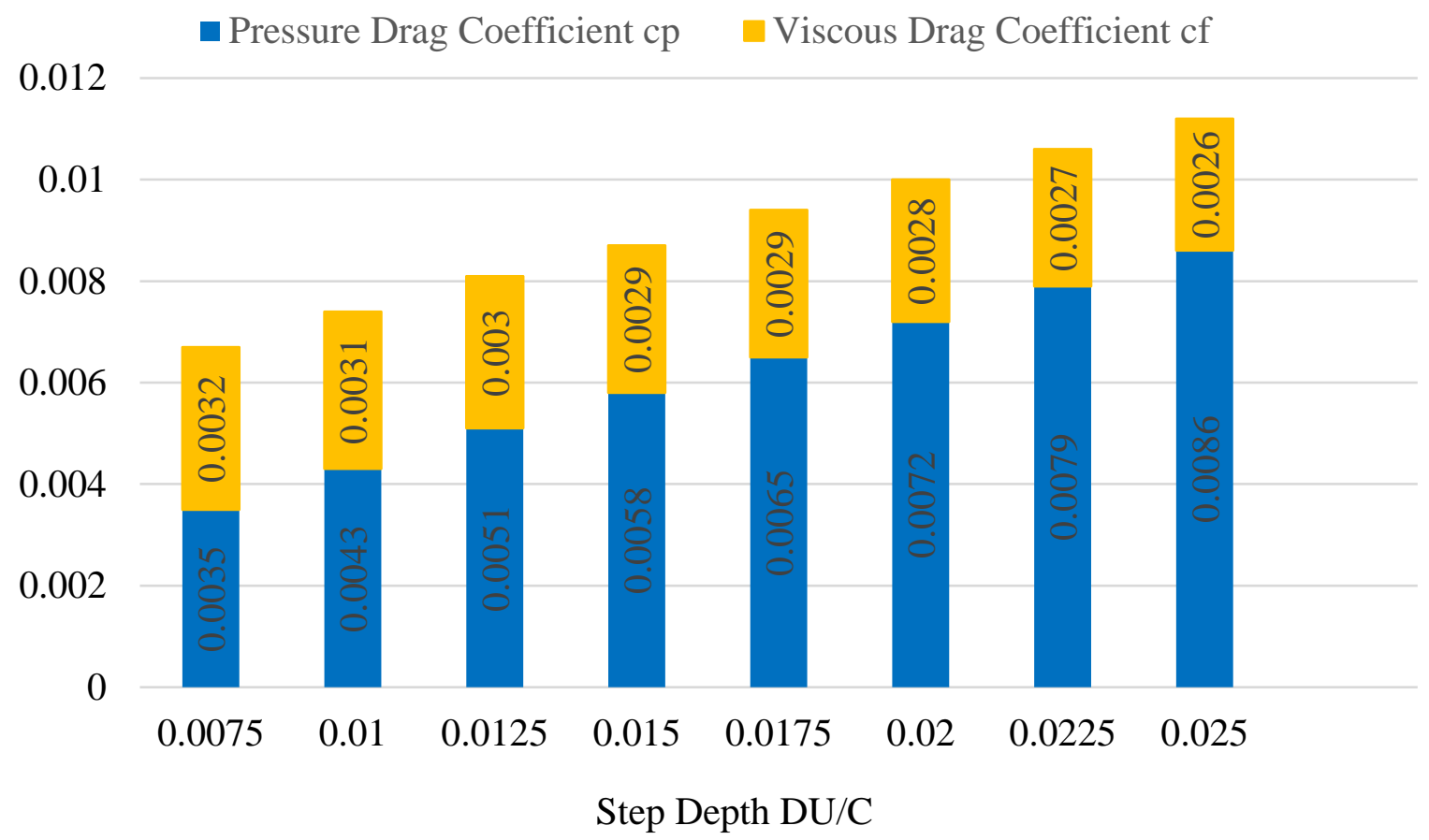

Figure 4.17. Decomposition of the Drag coefficient to pressure drag and viscous drag coefficients at different step depths.

Similar to the results shown in figure (4.11), the viscous drag component is nearly constant, while the pressure drag noticeably increases from one case to the other. This shows that the pressure drag is the dominant component in the case of stepped airfoils, and its contribution increases with the increase of the step depth. As the step depth increases, the pressure experiences a slight increase, but the exposed area of the airfoil noticeably increases. For that reason, the drag coefficient continuously and linearly increased as the step depth increased. 


\subsubsection{Effect of the step angle $\beta$}

In this subsection, the effect of the step angle on the aerodynamics of the stepped airfoil is studied. Figure (4.18) shows the five configurations of an airfoil with a backward-facing step at the midchord length, and a step depth of $D_{U}=0.015 C$, while the step angle $\beta$ changed from $45^{\circ}$ to $-45^{\circ}$, with the zero at the vertical position and positive angles are in the counter clockwise direction. In each case, the lift coefficient, drag coefficient and lift-to-drag ratio is calculated, and the results are tabulated in table (4.1).

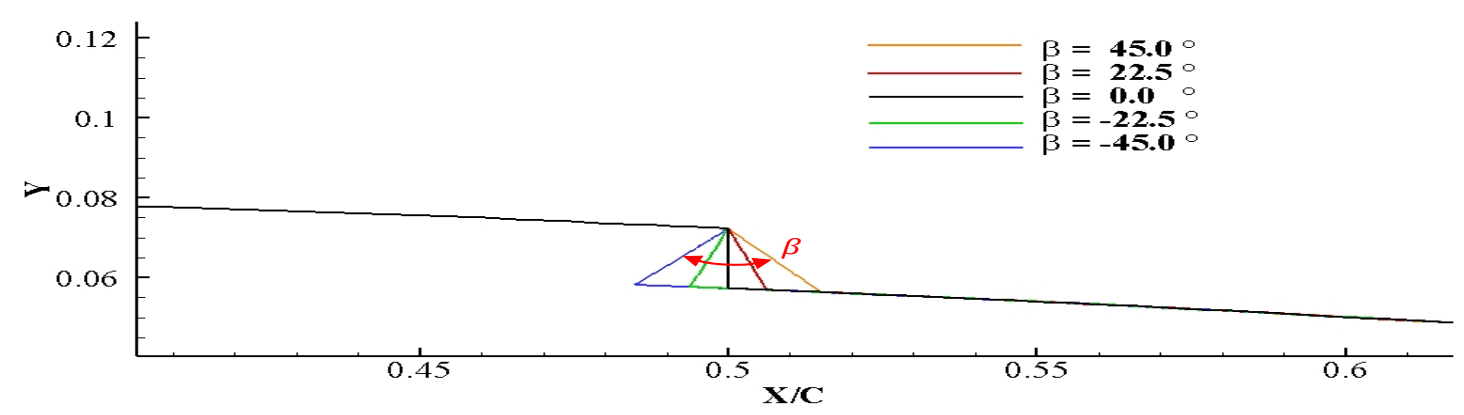

Figure 4.18. Different configurations of the NACA 2412 with different step angles.

Table 4.1. Values of the $\mathrm{cl}, \mathrm{cd}$ and $\mathrm{L} / \mathrm{D}$ at different step angles $\boldsymbol{\beta}$.

\begin{tabular}{lccc}
\hline Step Angle & $\boldsymbol{c}_{\boldsymbol{l}}$ & $\boldsymbol{c}_{\boldsymbol{d}}$ & L/D \\
\hline $\mathbf{4 5 . 0}^{\circ}$ & 0.44439 & 0.00878 & 50.6115 \\
$\mathbf{2 2 . 5}^{\circ}$ & 0.44494 & 0.00873 & 50.9527 \\
$\mathbf{0}^{\circ}$ & 0.44504 & 0.00873 & 50.9919 \\
$\mathbf{- 2 2 . 5}^{\circ}$ & 0.44503 & 0.00873 & 50.9779 \\
$\mathbf{- 4 5 . 0}^{\circ}$ & 0.44495 & 0.00873 & 50.9495 \\
\hline
\end{tabular}

The five cases obtained nearly the same values for the three aforementioned aerodynamic properties. A very small difference was observed in the lift-to-drag ratio $c_{l} / c_{d}$ in case of the angle $\beta=45^{\circ}$. This is due to a small displacement of the recirculation zone to the lower corner of 
the inclined step. In cases of step angles from $\beta=-45^{\circ}$ to $22.5^{\circ}$ the recirculation zone started at $X / C=0.513$, while in the case of $\beta=45^{\circ}$, the bottom corner of the step is shifted to $X / C=$ 0.517 , which is the location that marks the beginning of the recirculation zone. For cases with negative step angles, the additional space that is added to the step vicinity is filled with low-energy minute eddies that have negligible effect on the aerodynamics of the flow. Therefore, the effect of the step angle $\beta$ that is ranging between $45^{\circ}$ to $-45^{\circ}$ showed to be negligible on the aerodynamics of an airfoil with a backward-facing step on the upper surface of an airfoil. 


\subsection{Aerodynamics of Backward-facing Steps on the Lower Surface of an Airfoil:}

In this section, a detailed numerical simulation will be presented investigating the aerodynamic performance of airfoils with backward-facing step installed on the lower surface of a NACA 2412. The analysis starts with a mesh independence study using a family of five consecutively refined meshes to ensure a mesh independent numerical solution. The number of nodes is doubled from one mesh to the other, while the number of cells quadruples. The flow around the stepped airfoil was solved on the five meshes and values of the lift coefficient $c_{l}$ is compared from one mesh to the other. In figure (4.19), the lift coefficient values are plotted on the y-axis and the corresponding number of cells on the $\mathrm{x}$-axis.

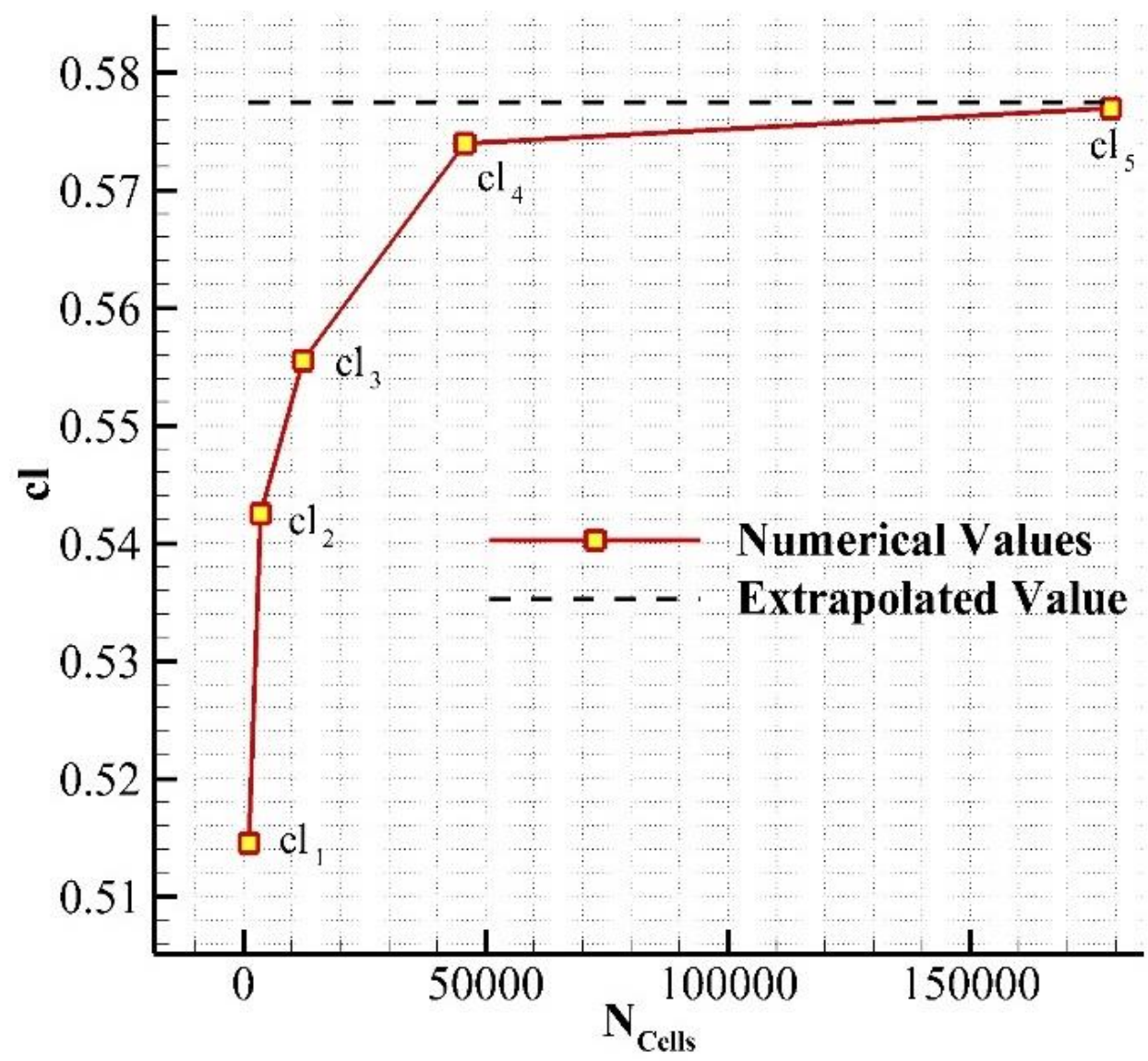

Figure 4.19. Convergence of the lift coefficient values as the mesh density increases.

As the number of cells increases, the calculated lift coefficient value approaches the asymptotic value that is theoretically achieved when the mesh spacing $h$ tends to zero $(h \rightarrow 0)$. This continuum value $c_{l(h=0)}$ was extrapolated using Richardson's method explained earlier and found equal to 0.5775 which shows that the result obtained from the finest mesh $\left(c_{l 5}\right)$ is about $0.1 \%$ off 
the continuum value $c_{l(h=0)}$. For that reason, the density of the finest mesh with about 180,000 cells and 340 nodes along the airfoil surface will be used to carry out all the numerical simulations in this study. All simulations carried out with the finest mesh obtained a Y+ value lower than 0.6. Therefore, the finest mesh will adequately ensure a mesh independent solution and is used in the following numerical analyses. 


\subsubsection{Effect of the step location $X_{L} / C$ :}

This subsection will present the effect of the step location on the aerodynamic properties of the stepped airfoil. The location of the step changed from $25 \%$ to $75 \%$ of the chord length with a $5 \%$ increment between a single configuration and the succeeding one.

\subsubsection{Effect of the Step Location on the Lift Coefficient $c_{l}$}

The variation of the lift coefficient value $c_{l}$ with the change of the step location is shown in figure (4.20). A first observation in figure (4.20) is that employing a step on the lower surface of the NACA 2412 has increased the lift coefficient when compared to the clean airfoil, regardless of the step location. At the same conditions, the clean airfoil achieved a lift coefficient value of 0.517 which is $11 \%$ lower than the lowest value obtained by the stepped configurations.

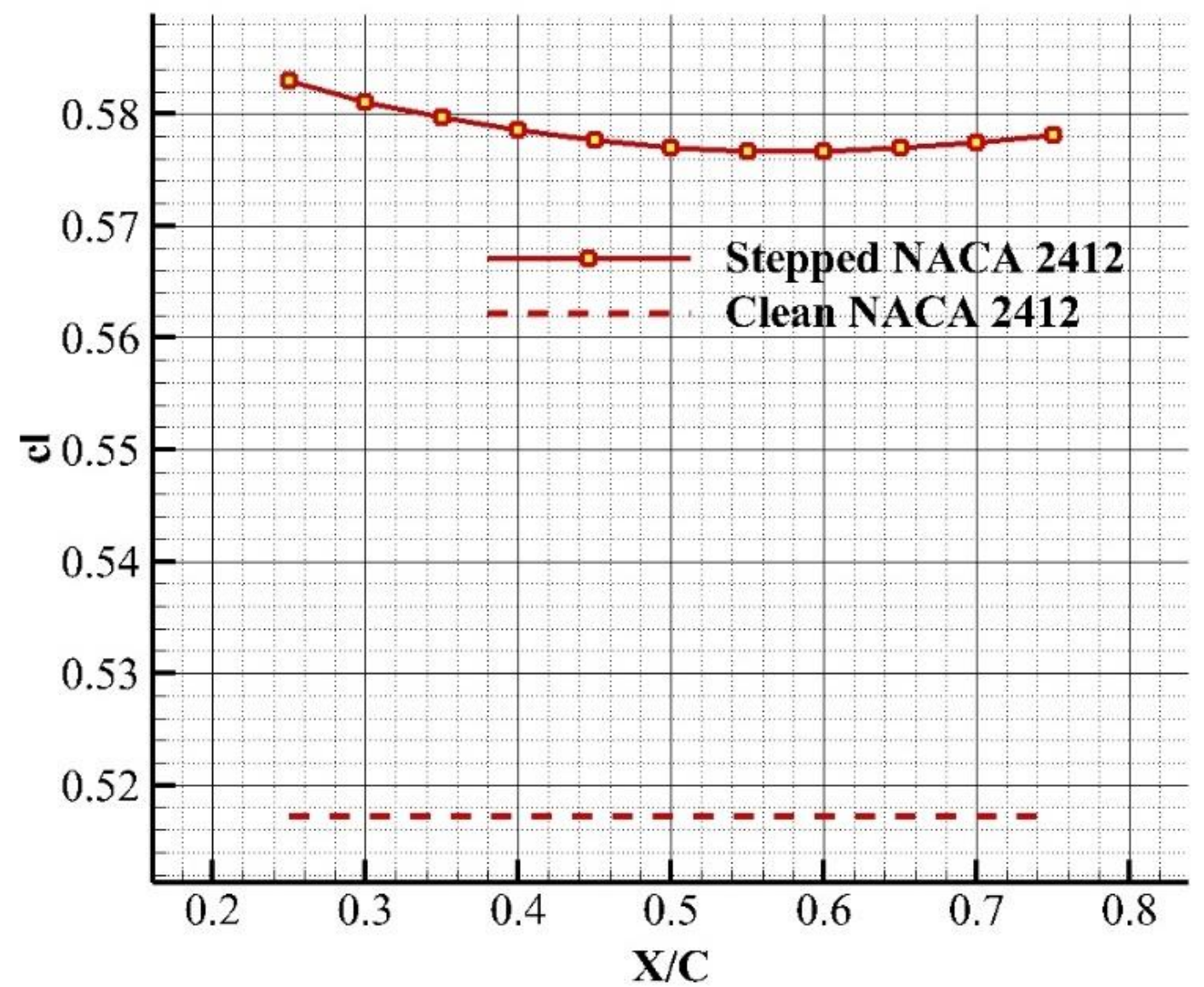

Figure 4.20. The lift coefficient value $c_{l}$ at different step locations $(\operatorname{Re}=5.7 \mathrm{e} 6, \mathrm{M}=0.2$, and $\alpha=$ $\left.2.5^{\circ}\right)$.

This increase in the lifting force is attributed to the sudden change in the airfoil thickness which will result in the deceleration of the flow at the step vicinity. The conservation of momentum 
dictates that a decrease in the velocity will cause the static pressure to increase on the pressure side, and since the total pressure did not decrease, the lifting forces acting on the airfoil significantly increased. The second observation in figure (4.20) is that the variation of the lift coefficient value with the step location did not follow a linear-like behavior as it was the case of a step on the upper surface, but rather a non-linear parabolic-like curve with a minimum value obtained for a configuration of a step at $X_{L} / C=0.55$. To understand this behavior, a closer look on the flow around the stepped airfoil is needed. Figure (4.21) shows the streamlines of the flow flooded with colors of the velocity magnitude. The backward-facing step resulted in a sudden reduction in the airfoil's thickness which forces the flow to locally separate at the edge of the step and create a low pressure recirculation zone as shown in figure (4.21).

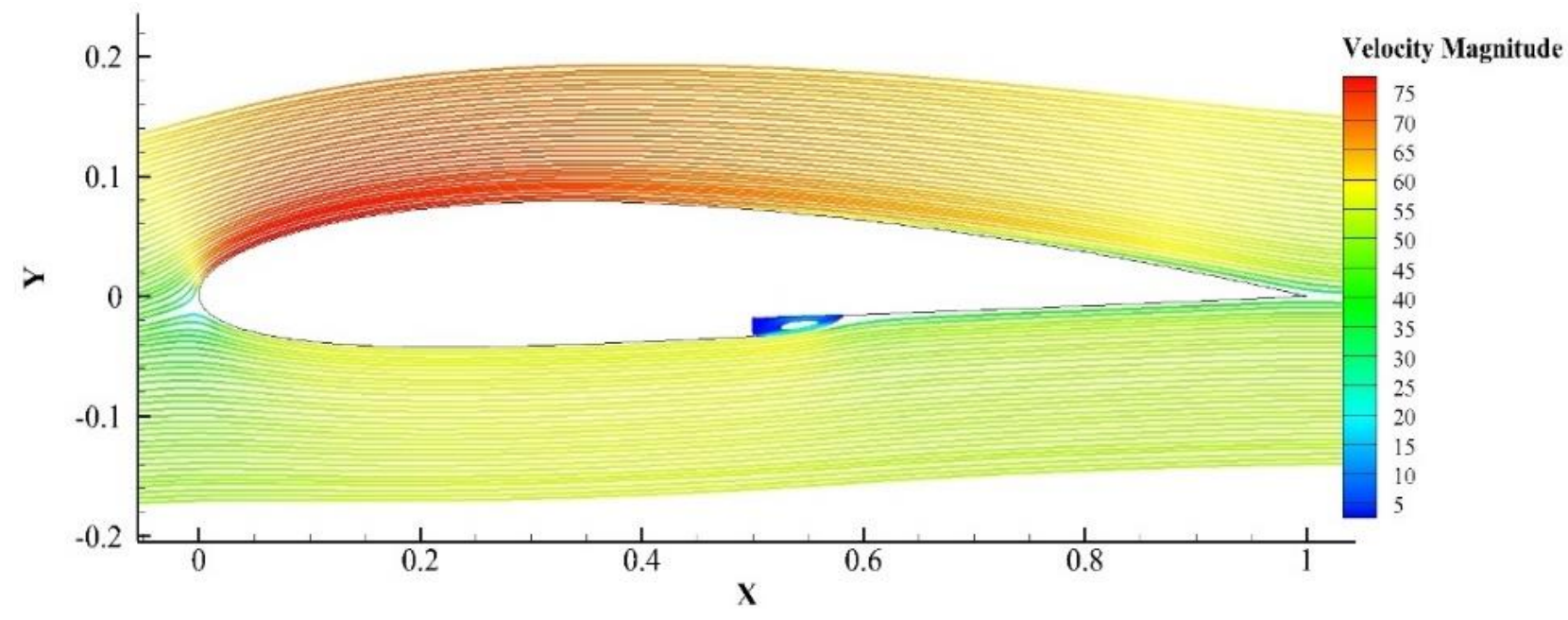

Figure 4.21. Streamlines of the flow around the stepped NACA 2412 showing its separation at the step edge, the creation of a recirculation zone and the further reattachment of the flow.

This low-pressure region assists the flow to reattach back to the thinned airfoil surface. In accordance with the conservation of mass, the reattached flow will be decelerated. Again, the conservation of momentum dictates that a decrease in the velocity field will result in an increase in the static pressure after the step. Therefore, in comparison to the clean airfoil, the pressure field will experience two additional variations; the first is a sudden reduction in the pressure field due to the presence of the recirculation zone, followed by an increase attributed to the change in the airfoil thickness. Figure (4.22) shows the pressure coefficient distribution over the lower surface of the clean NACA 2412, as well as in the eleven stepped configurations. By comparing the curve of the clean airfoil with cases of the stepped airfoil, the effect of the backward-facing step on the pressure distribution is clearly demonstrated. The separation of the flow at the step edge entrains a low-pressure recirculation zone, which decreases the lifting force of the airfoil. On the other hand, the change of the airfoil thickness will increase the pressure after the step and enhance the lifting force of the airfoil. 


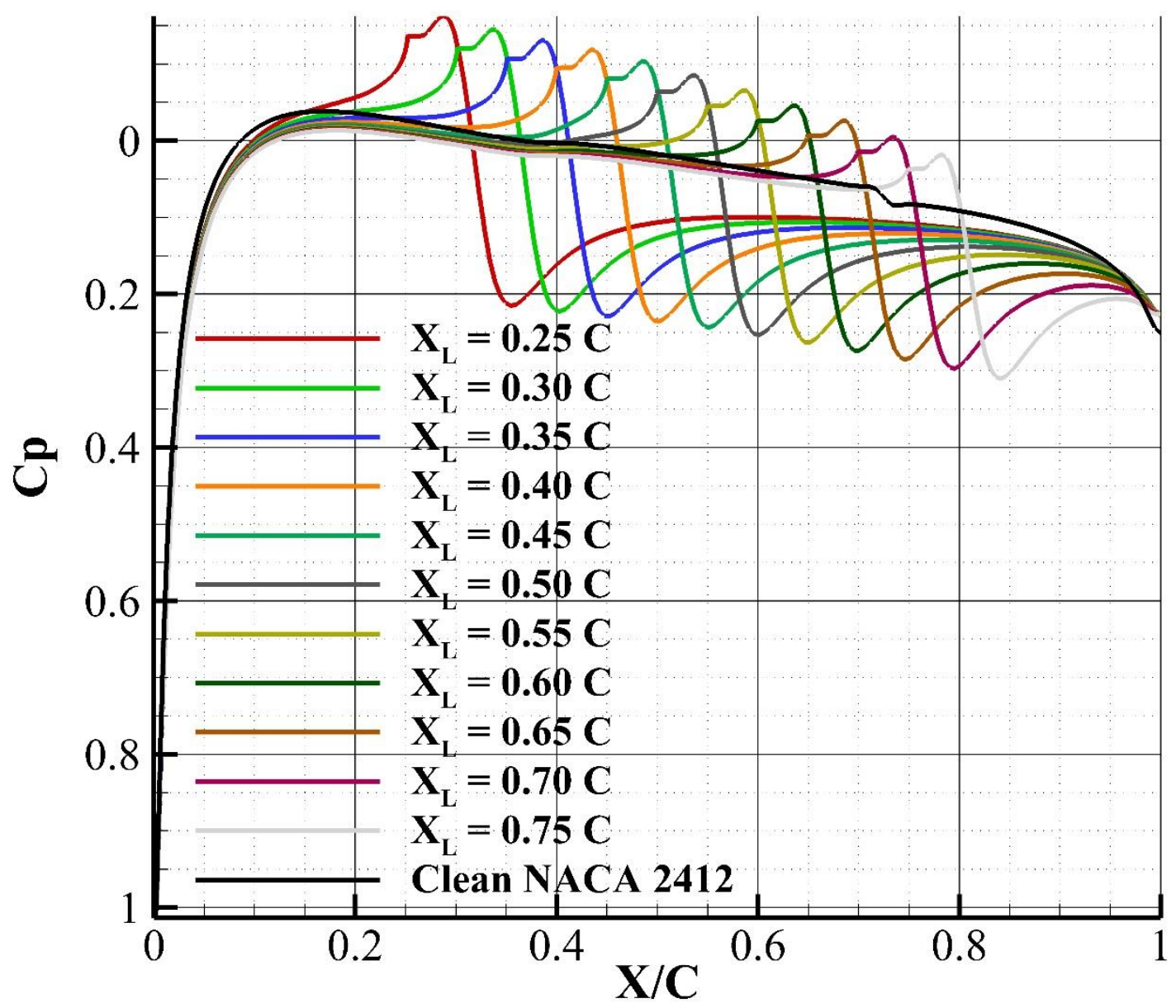

Figure 4.22. Pressure distribution over the lower surface of a clean NACA 2412 as well as in cases of stepped airfoil with steps at different locations $\left(\operatorname{Re}=5.7 \mathrm{e} 6, \mathrm{M}=0.2\right.$, and $\left.\alpha=2.5^{\circ}\right)$.

As the step location shifts from the leading edge towards the trailing edge, the area of the airfoil exposed to the high-pressure region decreases, leading to a drop in the lift coefficient value as shown in figure (4.20). However, for cases with a step located after $X_{L} / C=0.55$, a small increase in the lift coefficient values is observed. This is due to the thickness distribution of the NACA 2412 airfoil that promotes an increase in the pressure near the trailing edge of the airfoil. Therefore, as the step shifts towards the trailing edge, the pressure gradient along the airfoil supresses the intensity of the low-pressure recirculation zone and adds to the value of the high-pressure zone after the step. This leads to a slight increase in the value of the lift coefficient for cases with a step located after the point $X_{L} / C=0.55$. 


\subsubsection{Effect of the Step Location on the Drag Coefficient $c_{d}$}

In each of the eleven configurations of different step locations, the drag coefficient was calculated by resolving the normal and axial forces acting on the airfoil to a component parallel to the main stream velocity. Figure (4.23) shows the variation of the drag coefficient value as the step location shifts towards the trailing edge.

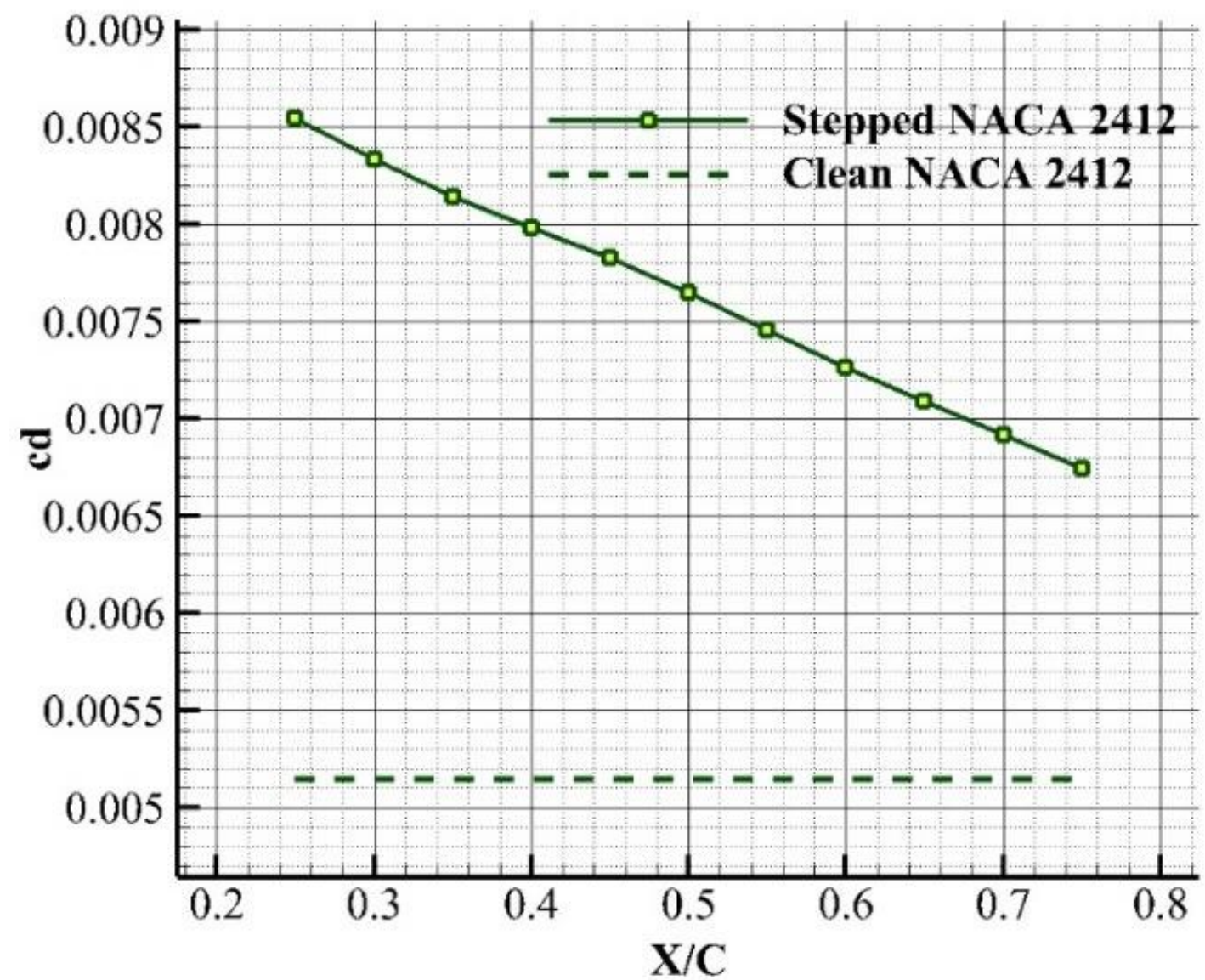

Figure 4.23. Pressure distribution over the lower surface of a clean NACA 2412 as well as in cases of stepped airfoil with steps at different locations $\left(\operatorname{Re}=5.7 \mathrm{e} 6, \mathrm{M}=0.2\right.$, and $\left.\alpha=2.5^{\circ}\right)$.

In all cases, the drag coefficient value is higher than 0.00515 , which is the value obtained by the clean airfoil at the same conditions. Equation (4.1) is again used to decompose the drag coefficient to its two main components. It should be noted here that for 2D subsonic flows, the wave drag and the induced drag components are inapplicable. Figure (4.24) shows the results of this decomposition in cases of the stepped airfoils as well as in case of a clean airfoil. 


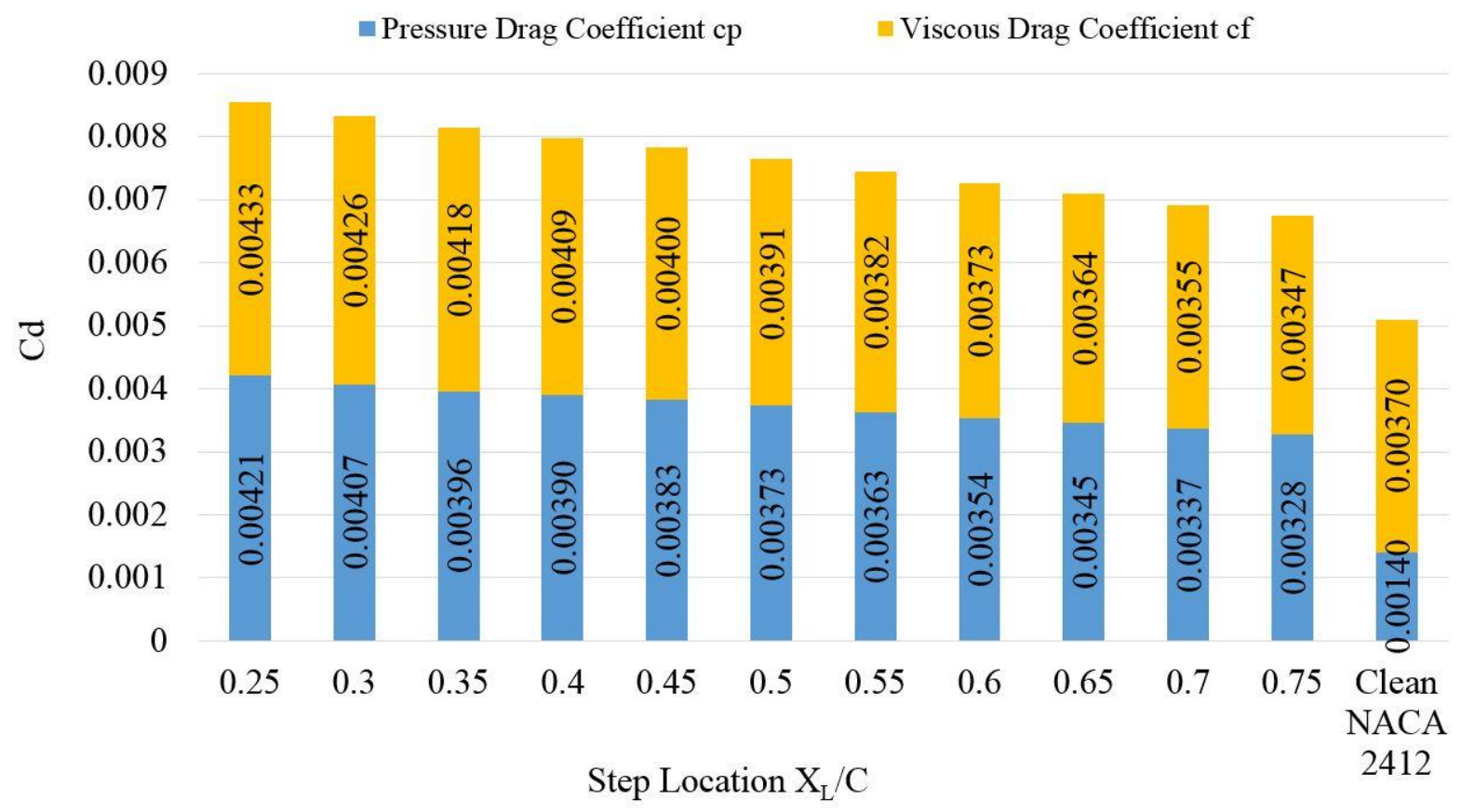

Figure 4.24. Decomposition of the drag coefficient to pressure drag and viscous drag coefficients $\left(\operatorname{Re}=5.7 \mathrm{e} 6, \mathrm{M}=0.2\right.$, and $\left.\alpha=2.5^{\circ}\right)$.

The decomposition of the drag coefficient in figure (4.24) explains the inverse relation between the drag coefficient and the step location shown in figure (4.23). As the step location shifts towards the trailing edge, both components of the drag coefficient decrease with decrements that are proportional to the step's shifted distance, but each component drops for a different reason.

The pressure drag coefficient is influenced by the static pressure value at the recirculation zone which acts directly on the vertical wall of the backward-facing step. As the step location shifts towards the trailing edge, the static pressure increases due to the decrease of the NACA 2412 thickness, which in turn increases the pressure at the recirculation zone. For that reason, the pressure drag coefficient value decreases as the step shifts from the leading edge towards the trailing edge.

On the other hand, the net viscous force is calculated as the integral of the shear stresses over the surface of the airfoil. To study the variation of the viscous drag coefficient values, the wall shear stress distribution over the airfoil (or the skin friction coefficient distribution) is presented in figure (4.25). 


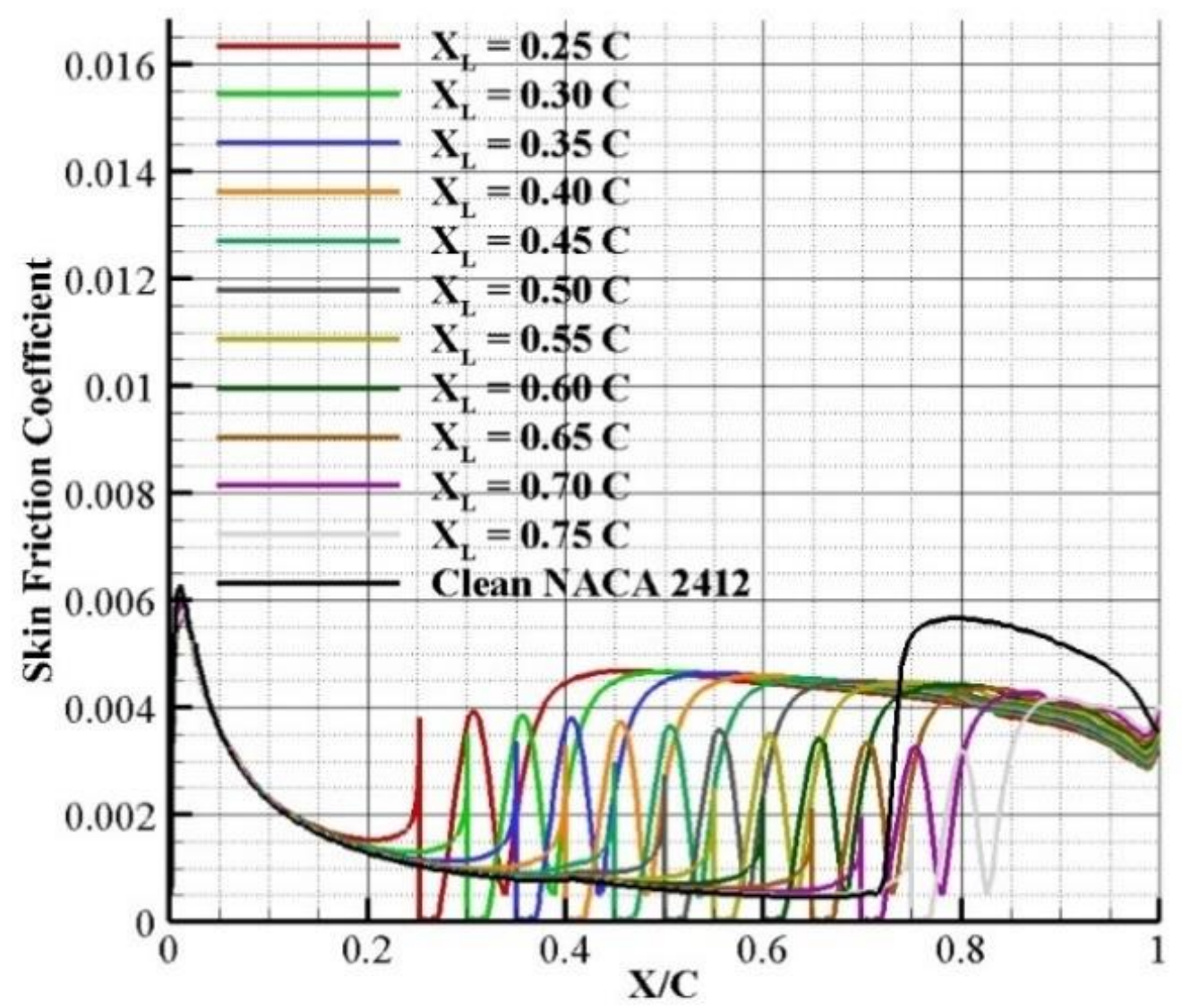

Figure 4.25. Skin friction coefficient distribution over the lower surface of a clean NACA 2412 as well as in cases of stepped airfoil with steps at different locations $(\operatorname{Re}=5.7 \mathrm{e} 6, \mathrm{M}=0.2$, and $\alpha$ $=2.5^{\circ}$ ).

In the case of the clean NACA 2412, the transition of the viscous boundary layer on the lower surface occurred naturally at about $73 \%$ of the chord length. As the step shifts towards the trailing edge, the area under the curves of the wall shear stress (or the skin friction coefficient in figure (4.25) decreases leading to a drop in the value of the viscous drag coefficient as shown in figure (4.24). With constant decrements in the pressure drag coefficient and viscous drag coefficient, the overall drag coefficient value decreases in a linear-like behavior as the step shifts towards the trailing edge.

Figure (4.25) shows another interesting observation which is the ability of some stepped configurations to delay the natural transition of the boundary layer from laminar to turbulent flow. In case of the clean airfoil, the viscous boundary layer experiences the transition at about $73.3 \%$ of the chord length, while in cases of the stepped airfoil, the transition occurs when the flow reattached after the recirculation zone. This was consistently observed in the last three configurations, where the step was located at $X_{L} / C=0.65,0.7$ and 0.75 , respectively. In these 
three cases, the transition occurred at about $X / C=0.74,0.78$ and 0.83 , respectively, which are after the natural transition point in the case of the clean airfoil. This delay resulted in lower values of the viscous drag component when compared to the clean NACA 2412. Figure (4.25) shows that the viscous drag coefficient of the clean airfoil was 0.00370 , while in the last three cases of the stepped airfoil, the viscous drag coefficient values were $0.00364,0.00355$ and 0.00347 , respectively.

\subsubsection{Effect of the Step Location on the Critical Angle of Attack $\alpha_{c r}$ :}

The near stall behavior of airfoils with backward-facing step on the lower side of the airfoil is studied using three stepped airfoil configurations with steps located at $X_{L} / C=0.25,0.5$ and 0.75. These configurations are tested and compared to the clean NACA 2412. Figures (4.26 a) and (4.26 b) show the values of the lift coefficient $c_{l}$ and drag coefficient $c_{d}$ at different angles of attacks. In the three configurations of the stepped airfoil, the maximum lift, and thus the critical angle of attack $\alpha_{c r}$ was at $16^{\circ}$. On the other hand, the clean airfoil achieved the maximum lift at a critical angle of attack of $18^{\circ}$. It is speculated that this is due an alternation in the pressure distribution on the upper surface of the airfoil. This alteration is due to trailing edge effects of the flow that is influence by step location on the lower surface.

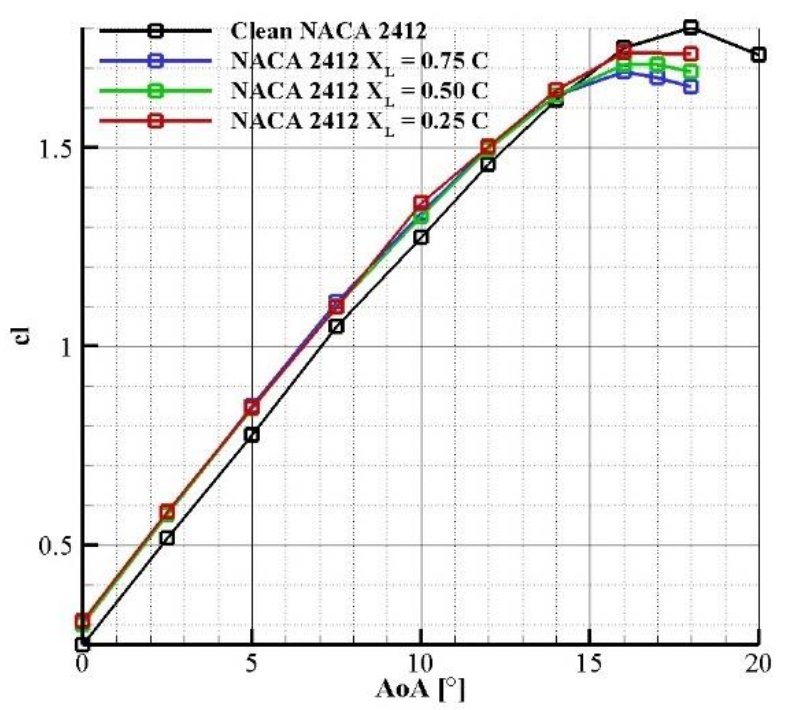

(a)

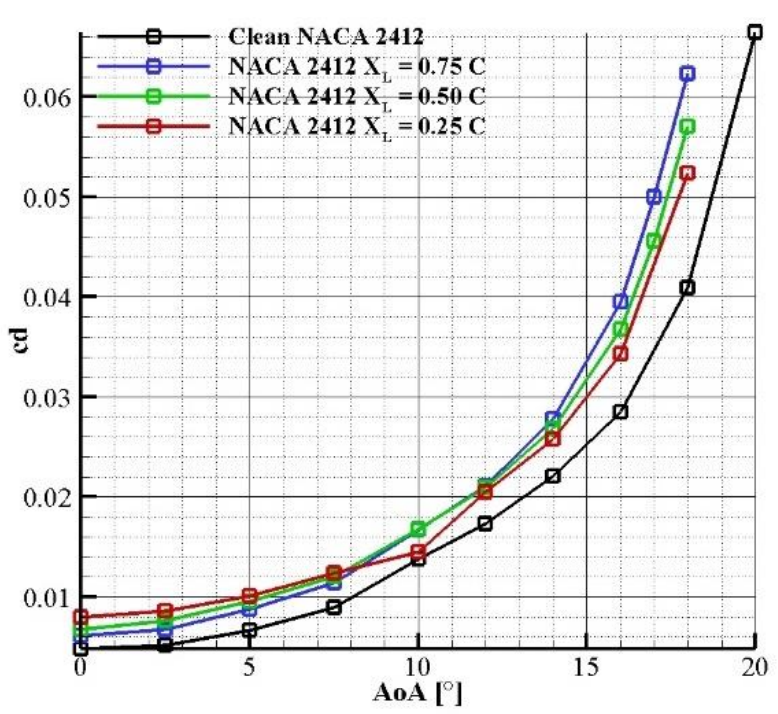

(b)

Figure 4.26. (a) Lift and (b) drag coefficients versus angle of attack $\alpha(\operatorname{Re}=5.7 \mathrm{e} 6$ and $\mathrm{M}=$ $0.2)$.

The effect of the early stall behavior of the stepped configurations is manifested in figure (4.26 b) which shows the amplified drag coefficient values as the flow approaches stall. This means that 
employing a step on the pressure side of an airfoil has an adverse effect on the onset of stall, and the step location has a negligible effect on the critical angle of attack $\alpha_{c r}$. 


\subsubsection{Effect of the step depth $D_{L} / \mathrm{C}$}

This subsection studies the effect of the step depth on the aerodynamics of an airfoil with a backward-facing step installed on its lower surface. Eight different step depths are examined, starting from $D_{L} / C=0.0075$ to $D_{L} / C=0.025$, with an increment of $0.0025 C$ from one configuration to the other. The step is fixed at the mid chord-length of the airfoil, and the flow is directed at an angle of attack of $2.5^{\circ}$. In each case the flow is solved numerically, and the lift coefficient $c_{l}$ and the drag coefficient $c_{d}$ are calculated to establish a correlation between these aerodynamic properties and the backward-facing step depth.

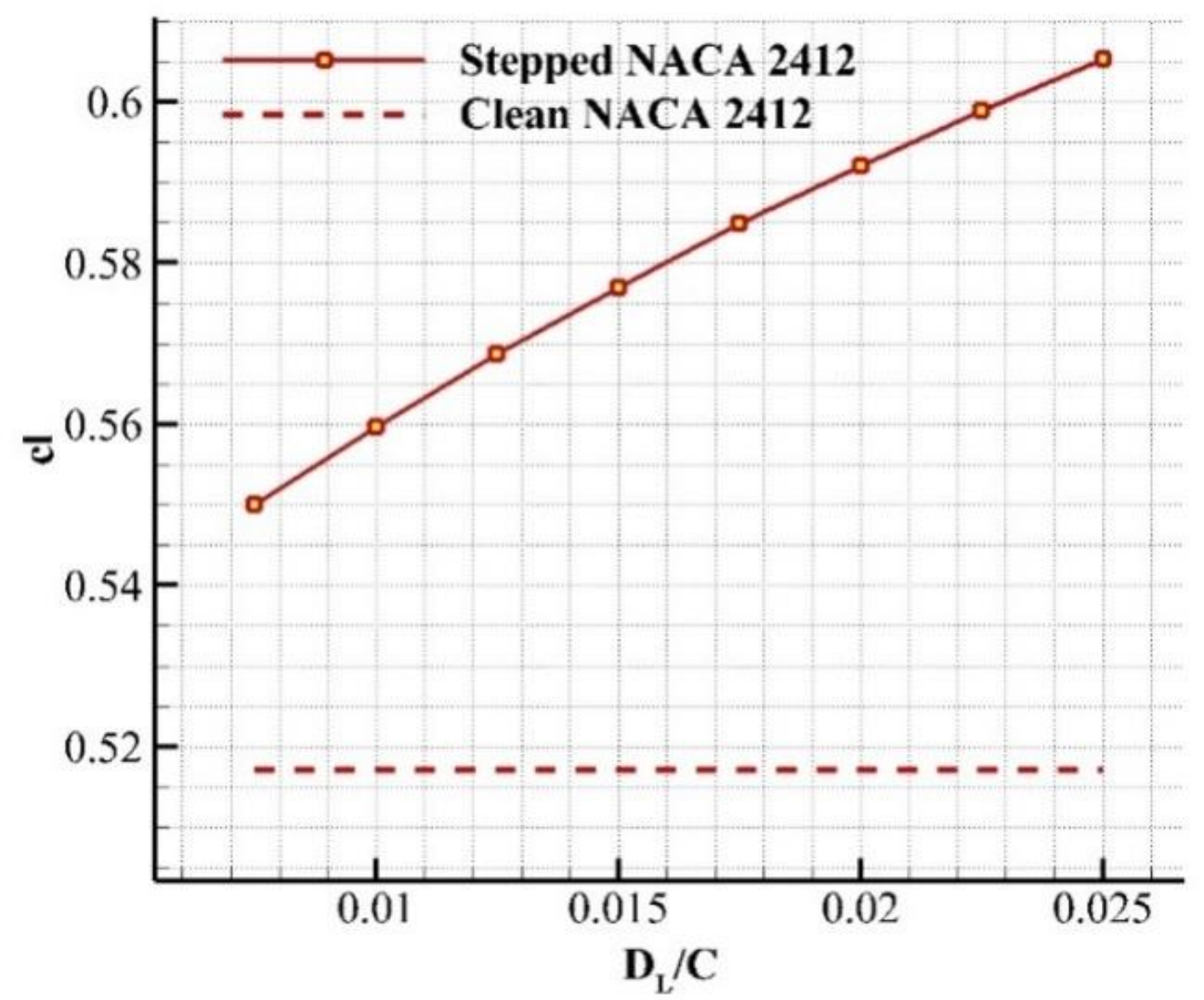

Figure 4.27. The lift coefficient value $c_{1}$ at different step depths $(\mathrm{Re}=5.7 \mathrm{e} 6, \mathrm{M}=0.2$, and $\alpha=$ $\left.2.5^{\circ}\right)$.

The lift coefficient was observed to increase by approximately $10 \%$ from 0.55 to 0.62 as the step increased from $D_{L} / C=0.0075$ to $D_{L} / C=0.025$, respectively as shown in figure (4.27). All the lift coefficient values obtained from stepped airfoils with lower surface step are higher than 0.517 
which is the value obtained from the clean NACA 2412 at the same boundary conditions. Despite this noticeable improvement in the lifting capabilities of the airfoil, installing the backward-facing step on the lower surface has perturbed the streamlined profile of the airfoil, and induced an additional form drag component that adds to the total drag force on the airfoil. Figure (4.28) shows the variation of the drag coefficient value from one configuration to the other. The drag coefficient values of the stepped airfoil experienced a noticeable increase that is directly proportional to the step depth.

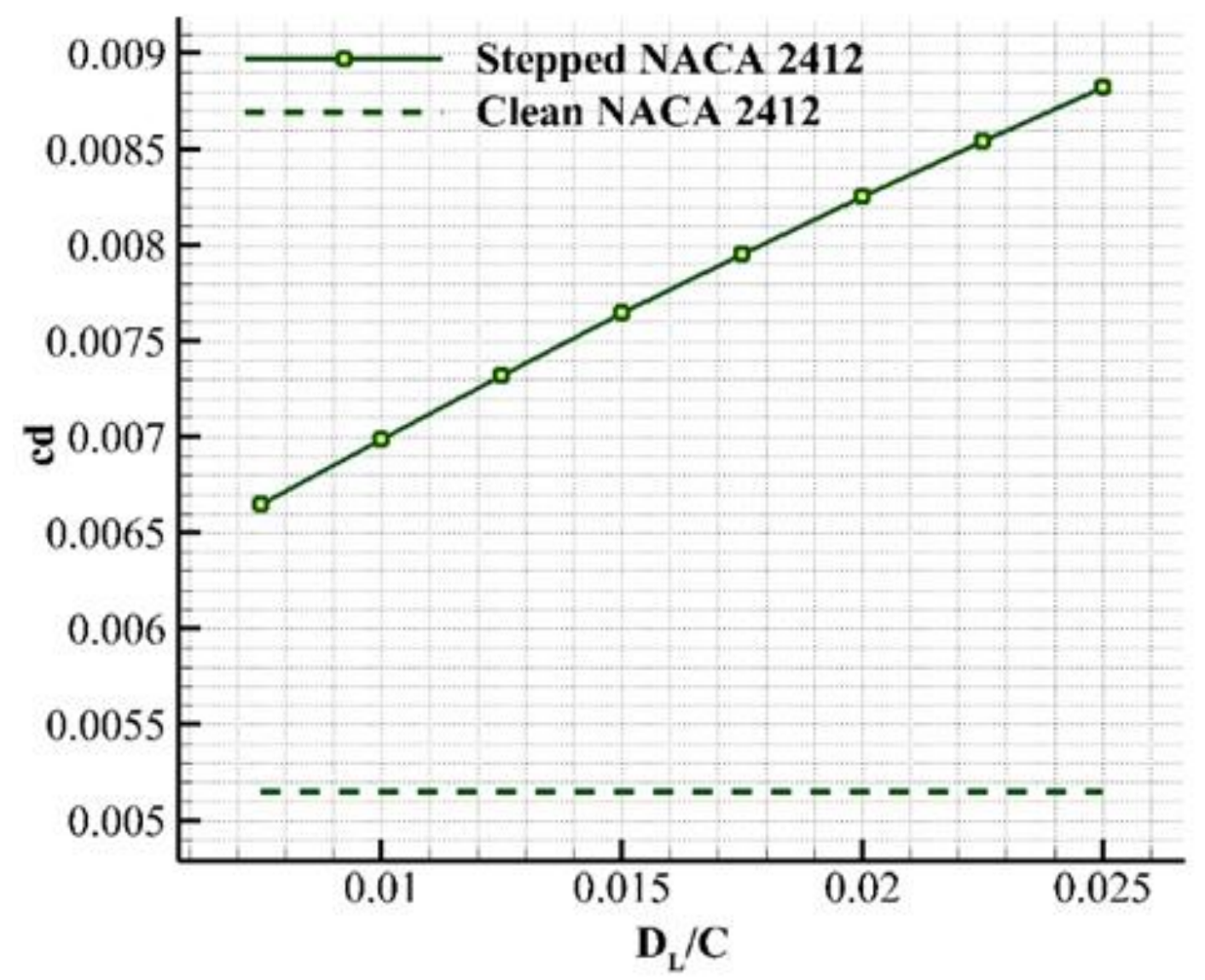

Figure 4.28. The drag coefficient value $c_{d}$ at different step depths of a backward facing step installed on the lower surface of a NACA 2412 airfoil $\left(\operatorname{Re}=5.7 \mathrm{e} 6, \mathrm{M}=0.2\right.$, and $\left.\alpha=2.5^{\circ}\right)$.

The smallest step depth of $D_{L} / C=0.0075$ resulted in an approximately $29 \%$ increase in the value of the drag coefficient when compared to the value obtained by the clean airfoil. This increase in the drag value grows with the step depth to reach approximately $71.3 \%$ in the case of a step depth of of $D_{L} / C=0.025$. The decomposition of the drag forces into its two components, namely, viscous drag and pressure drag is shown in figure (4.29). 


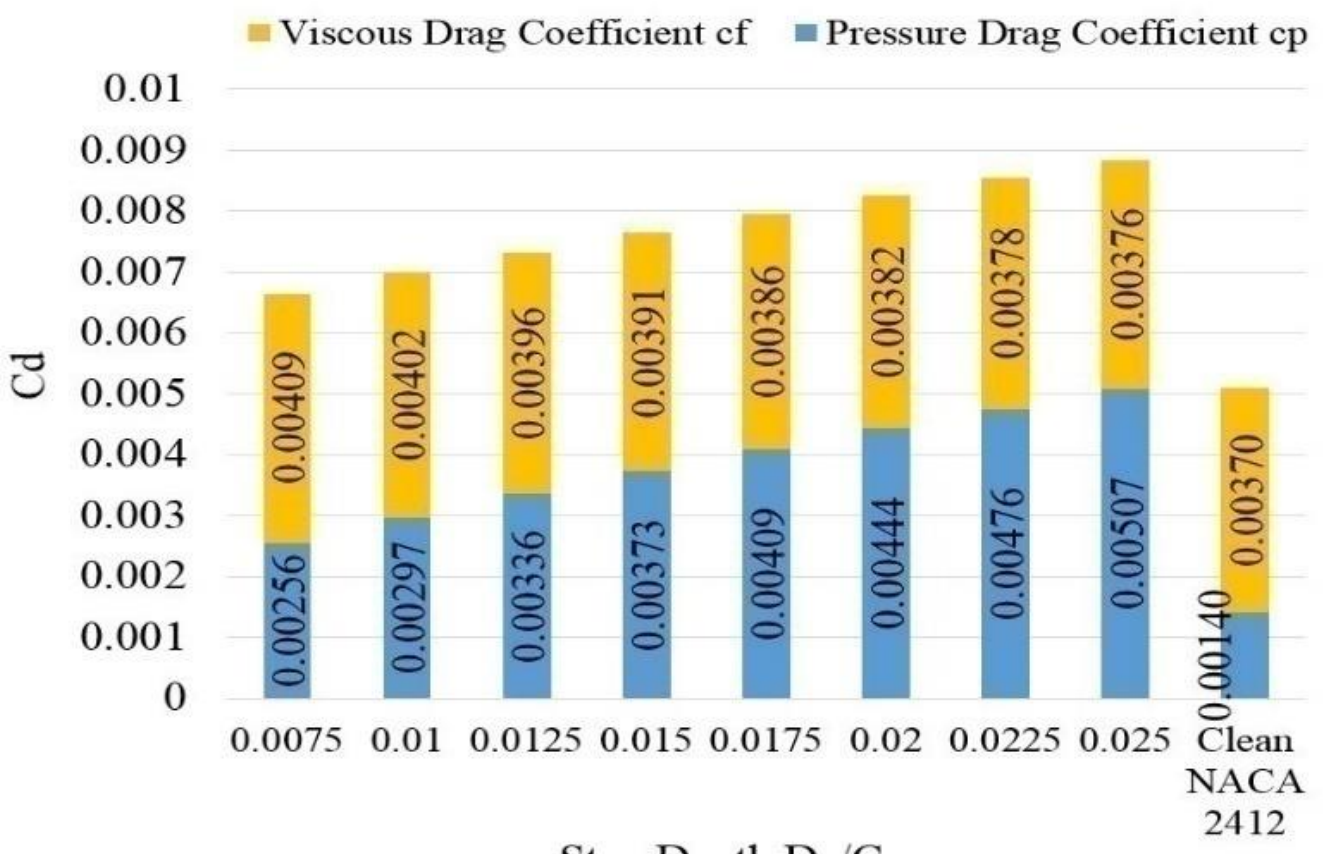

Step Depth $\mathrm{D}_{\mathrm{L}} / \mathrm{C}$

Figure 4.29. Decomposition of the drag coefficient to pressure drag and viscous drag coefficients at different step depths.

As the step depth increases, the viscous drag force slightly drops, while the pressure drag component increases significantly. The viscous drag is dropping slightly with increased step depth because larger steps produce more entropy at the recirculation zone which results in more energy dispersal, hence, lower turbulent kinetic energy after the flow reattachment. This drop in the turbulent kinetic energy is translated to lower wall shear stresses and consequently lower skin friction coefficient values as shown in figure (4.29). The pressure drag increases with the step depth for the same reason mentioned in subsection 4.2.2. As the step depth increases, the lowpressure recirculation zone acts on a larger surface area resulting in larger drag force acting on the airfoil. The increase in the pressure drag overshadows the slight decrease in the viscous forces resulting in an overall increase in the drag coefficient value with the increase of the step depth as shown in figure (4.28).

\subsubsection{Effect of the step angle $\beta$}

Similar to the analysis shown in subsection 4.2.3 where the effect of the angle of the upper surface backward-facing step was investigated, the same procedure is followed in this subsection, but this time the step is installed on the lower surface of a NACA 2412 airfoil. The step location is fixed at the mid-chord length with a depth of $D_{L}=0.015 C$, and an angle ranging from $\beta=45^{\circ}$ to $\beta=$ $-45^{\circ}$, where a positive angle is measured from the lower edge of the step and in the counter- 
clockwise direction. In each of the five cases, the lift coefficient, drag coefficient and lift-to-drag ratio are calculated and tabulated in table 4.2.

Table 4.2. Values of $\mathrm{cl}, \mathrm{cd}$ and $\mathrm{L} / \mathrm{D}$ at different step angles.

\begin{tabular}{cccc}
\hline $\boldsymbol{\beta}$ & $\boldsymbol{c}_{\boldsymbol{l}}$ & $\boldsymbol{c}_{\boldsymbol{d}}$ & $\boldsymbol{c}_{\boldsymbol{l}} / \boldsymbol{c}_{\boldsymbol{d}}$ \\
\hline $\mathbf{4 5 . 0}^{\circ}$ & 0.57703 & 0.0076517 & 75.41296 \\
$\mathbf{2 2 . 5}^{\circ}$ & 0.57698 & 0.0076474 & 75.44916 \\
$\mathbf{0}^{\circ}$ & 0.57697 & 0.0076459 & 75.46084 \\
$\mathbf{- 2 2 . 5}^{\circ}$ & 0.57699 & 0.0076473 & 75.45039 \\
$\mathbf{- 4 5 . 0}^{\circ}$ & 0.57706 & 0.0076571 & 75.36398 \\
\hline
\end{tabular}

As observed in subsection 4.2.3, changing the step angle has a negligible effect on the lift coefficient, drag coefficient and lift-to-drag ratio. The five configurations obtained the same pressure distribution curves and the same skin friction coefficient curves. In all cases, the recirculation zone started at $X / C=0.52$ and ended at $X / C=0.585$ even at a step angle of $\beta=$ $45^{\circ}$ which creates the largest vicinity at the step corner. The additional space is filled with a cascade of eddies trapped in the step's corner and their length scales decrease gradually from one level to the other. These eddies are known as the Moffatt eddies, and theoretically there should be an infinite number of these vortices trapped at the step corner as their length scale drops to zero. As the resolution of the computational domain increases, more Moffatt eddies can be modeled. Figure (4.30) shows the streamlines of the Moffatt eddies in each of the five configurations at different step angles.

As shown in figure (4.30), the main recirculation zone has the same structure and dimensions in all the five cases. As the step angle varied from $\beta=-45^{\circ}$ to $\beta=45^{\circ}$, an additional space is created at the step corner. This additional space is filled with the low energy Moffatt eddies whose influence on the airfoil aerodynamics is negligible. Four levels of the Moffatt eddies were modeled in case of a step angle of $\beta=45^{\circ}$, and the number of eddies' levels decreases with the step angle as shown in figure (4.30). Only the main recirculation zone has influence on the aerodynamics of the airfoil, and since the main recirculation zone has the same structure in the five configurations, the lift coefficient, drag coefficient and lift-to-drag ratio are not influenced by the variation of the step angle $\beta$. 

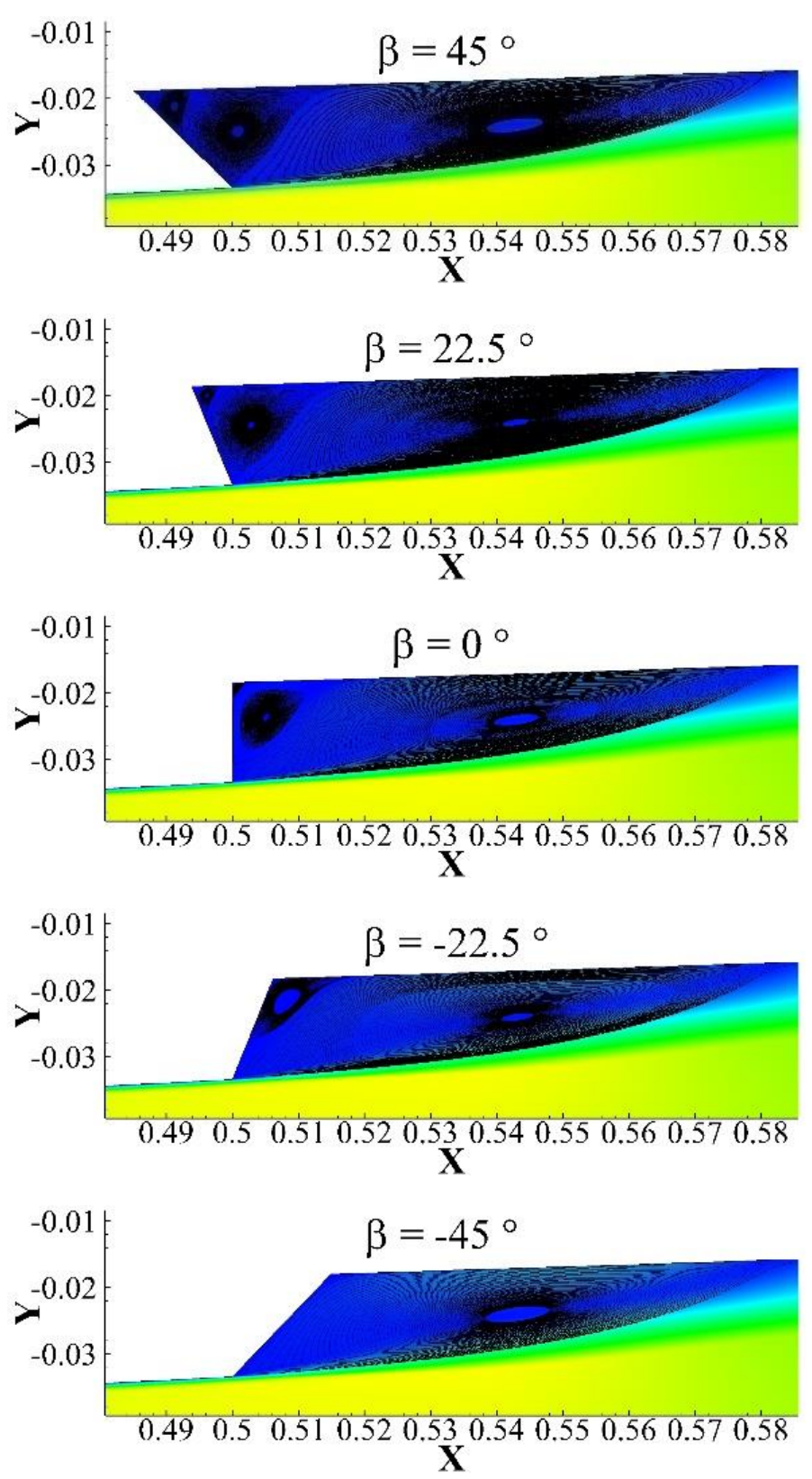

Figure 4.30. Streamlines of the recirculation zone and the Moffatt eddies at different step angles.

Studying sliding panels as a solution for the morphing skin problem has showed that the discontinuity introduced between the panels have imposed an aerodynamic penalty on the performance of this morphing design. The only case where the aerodynamic performance matched that of the clean airfoil is when the panels are separated by gaps that follow the condition $\frac{L}{\theta} *$ 
$\sqrt{R e_{\theta}}<600$. Unfortunately, this condition is not easily fulfilled at different morphing motions as shown in figure (1.5). For that reason, a seamless smooth design of morphing skin will be presented in chapter 5 . The design of a flexible morphing skin and its aerodynamics will be presented. 


\section{DESIGN AND AERODYNAMICS OF FLEXIBLE MORPHING SKIN}

\subsection{Design of The Flexible Morphing Skin}

\subsubsection{The Underlying Substructure:}

Another viable solution for the compromising problem of morphing skins is the use of flexible elastomer reinforced by fibers and an underlying structure. Elastomers are polymer-based materials made out of long chains of carbon, hydrogen and oxygen atoms. Each chain is crosslinked to its neighbouring chains at different points. The cross-linkage has provided the elastomers with high elasticity ranging from $200-1000 \%$ depending on the material used. Elastomers are also known for their high resilience which ensures a quick restoration to its original shape after the deforming force has been removed. These advantages are added to their very low permeability to gases and liquids, their electric and thermal insulation, and their ability to adhere to different fibers and metals.

The current study will take an advantage from this concept and use a specially modified zeroPoisson ratio cellular structure as an underlying support for the skin while the major ribs of the mesh will provide the module with the required stiffness along the chord-wise direction. The mesh will be designed with high flexibility in the desired degrees of freedom to ensure minimal actuation requirements. A highly anisotropic elastomer composite will be used as a sealant for the mesh. This outer layer will be tailored to maintain high stiffness along the chord-wise direction with the usage of reinforcing fibers and metal strips. Out-of-plane deflection of the skin will be experimentally tested by applying normal loads on the skin. 


\subsubsection{Design of the Underlying Substructure:}

Cellular structures are widely used in aerospace applications for their low density, low in-plane actuation requirements and high out-of-plane stiffness. Conventional honeycomb meshes inherit positive Poisson ratio resulting in a contraction of the mesh in a direction normal to the applied force as shown in figure (5.1).

\section{Convectional Honeycomb Mesh}
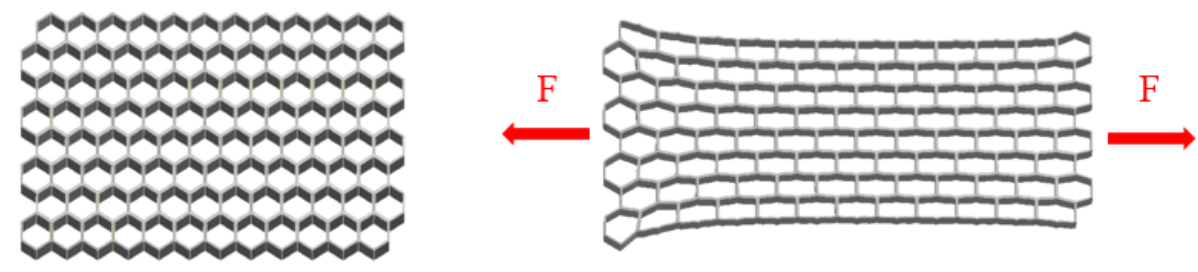

Zero Poisson Ratio Accordion Mesh
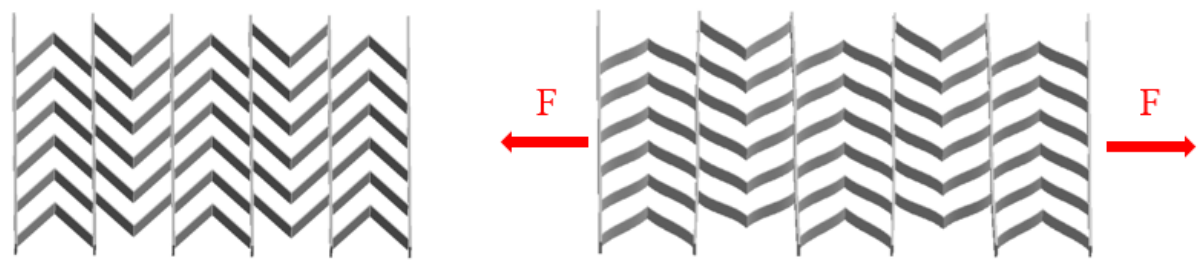

Figure 5.1. Geometrical change of conventional mesh compared to zero-Poisson ratio accordion mesh.

To overcome this disadvantage, a zero-Poisson ratio with accordion mesh will be used to provide the required motion without experiencing any contraction along the dimension normal to the applied force as shown in figure (5.1). Proper choice the geometric parameters of the accordion mesh shown in figure (5.2) will result in a viable substructure for a four-degree of freedom morphing skin. In the next few lines, these parameters will be determined according to the required skin kinematics without sacrificing strength in the desired directions.

\subsubsection{The Supporting Ribs:}

The accordion mesh consists of V-shaped minor ribs separated by major ribs along the chord-wise direction. These major ribs will provide the required stiffness and maintain the airfoil shape constant along the wing. Aluminum rods will be used as a reinforcement for the ribs as shown in figure (5.3). Aluminum was chosen for its high specific strength and workability where these rods will be bent to the desired airfoil of the wing. 


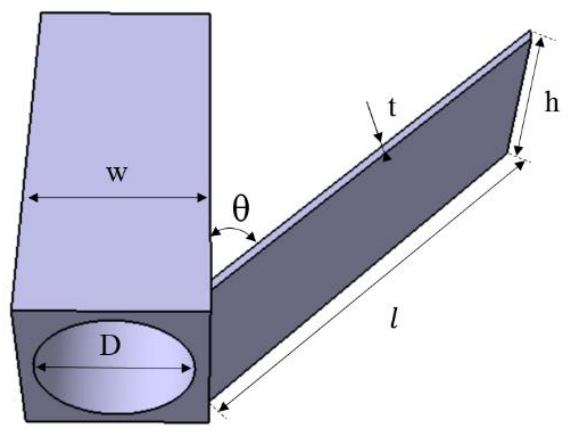

Figure 5.2. Geometric parameters of a single element of a zero-Poisson ratio accordion mesh.

When aerodynamic loads are applied on the wing, the major ribs will experience small deflections that could be estimated by solving the Euler-Bernoulli beam equation (5.1) for a fixed - fixed beam.

$$
E_{A l} I \frac{d^{4} \delta}{d x^{4}}=q
$$

Where $E_{A l}$ is the Young's modulus of aluminum, $I$ is the second moment of area of the cylindrical rods, and $q$ is the distributed load. In turn, the ribs need to be supported using spars where the separating distance $L$ between the spars will depend mainly on diameter of the aluminum rods.

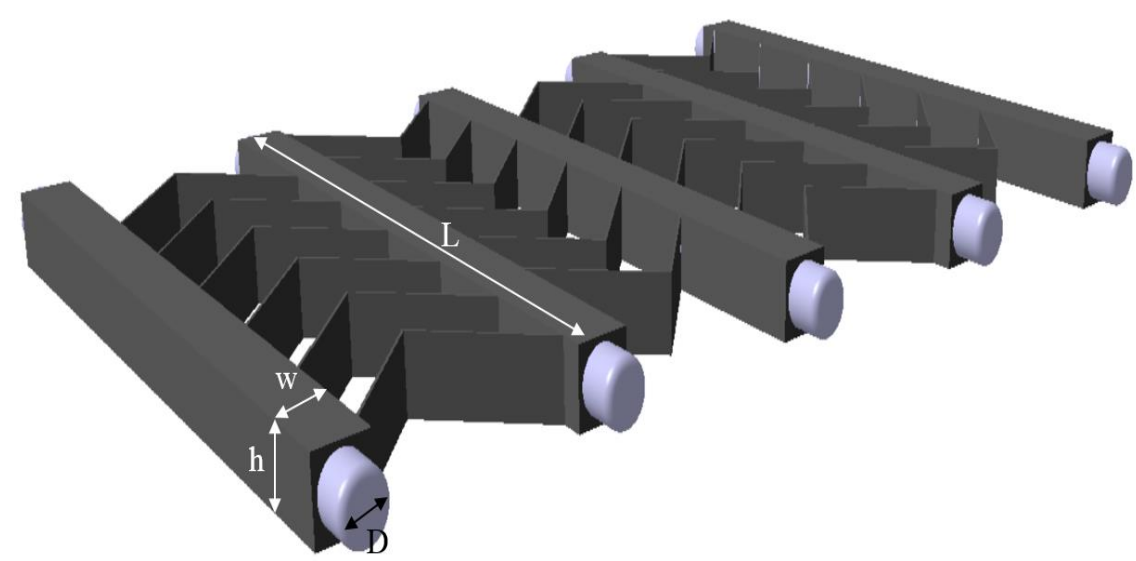

Figure 5.3. Aluminum rods supporting the accordion mesh to minimize the out-of-plane deflection. 
With a hypothetical choice of the pressure on the wing of $\mathrm{P}=10 \mathrm{kPa}$ and a maximum designed deflection for the aluminum rods of $\delta_{\max }=1 \mathrm{~mm}$, equation (5.1) is solved to obtain:

$$
L=\sqrt[3]{\frac{3 \delta_{\max } E_{A l} \pi D^{4}}{4 P(D+\sqrt{2} l)}}
$$

which represents the relationship between the separating distance of the spars $L$ and the diameter of the aluminum rods $D$.

This relationship is plotted in figure (5.4) showing the required diameter of the aluminum reinforcement rods for different spars' separating distances. Depending on the diameter of the rods, the height $h$ and the width $w$ of the major ribs in the mesh could be chosen to be a few millimeters larger than the diameter D. A diameter of $1 / 2$ in is chosen for the prototype used in this study, therefore, the height and the width are chosen to be $h=w=15 \mathrm{~mm}$.

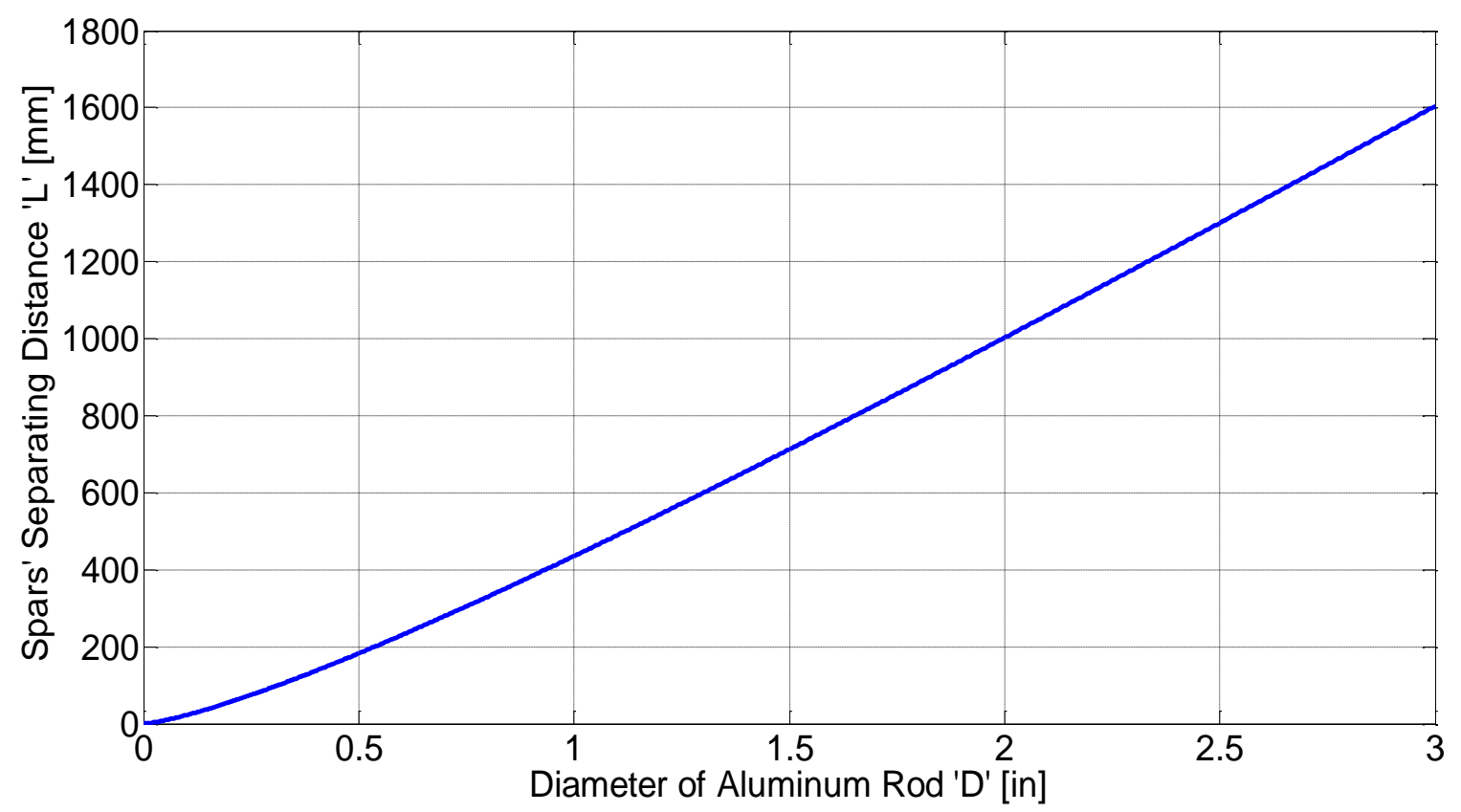

Figure 5.4. Relationship between the separating distance of the spars and the diameter of the supporting rods. 


\section{In-Plane Motion:}

The morphing wing is capable of performing two independent in-plane motions. These motions results in changing the span and the sweep angles of the wing. Most of the morphing skin will experience these two motions as a tensile and compressive loading when changing the wing's span and a shear loading when changing the sweep angle. The main intent of a passive morphing skin is to maximize the range of these motions while keeping the actuation requirements to a minimum. This could be achieved by calculating an equivalent Young's modulus $E_{e q}$ and shear modulus $G_{e q}$ for the whole mesh.

Gibbson and Ashbey [84] used the Euler-Bernoulli beam theory to calculate an equivalent in-plane Young's modulus for conventional honeycomb meshes and the same concept was used in [85]. Following a similar procedure, $E_{e q}$ and $G_{e q}$ can be evaluated for the accordion mesh.

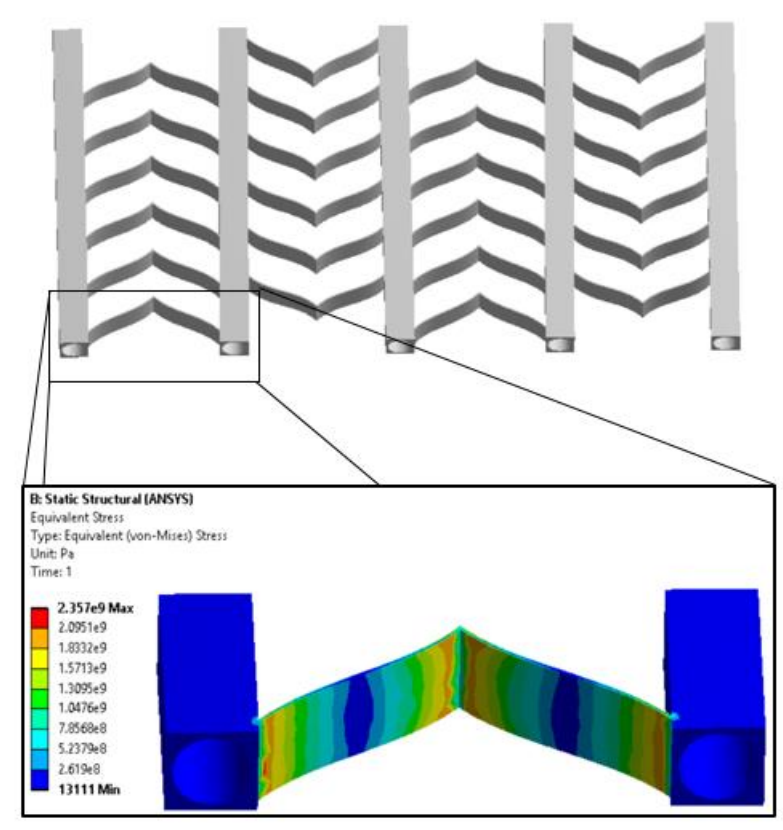

(a)

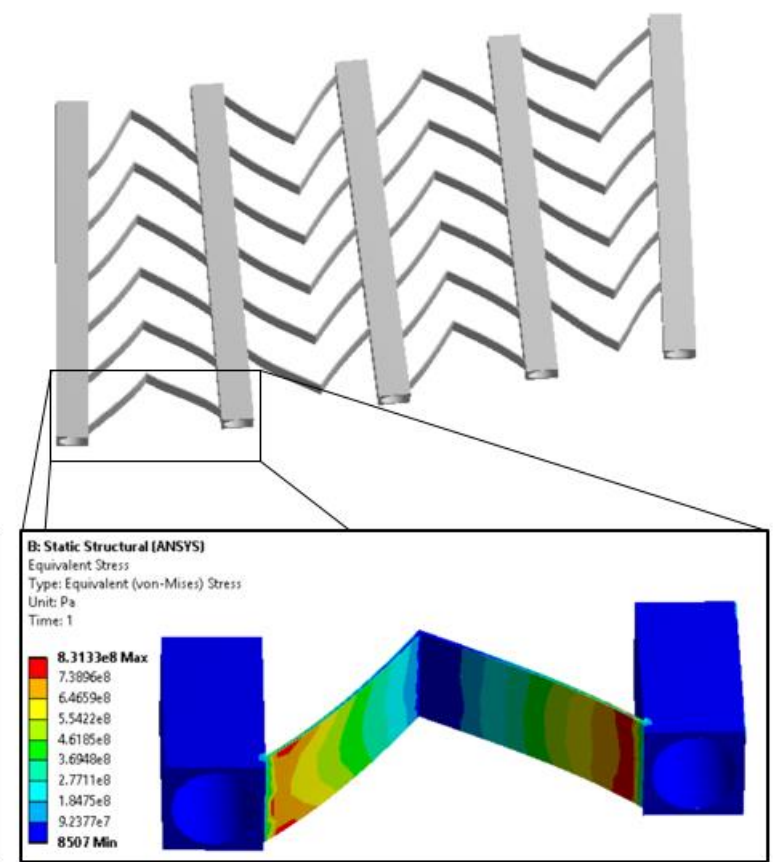

(b)

Figure 5.5. The morphed accordion mesh when experiencing (a) tensile loading and (b) shear loading with the accompanying Von-Misses stresses calculated using FEA.

If a force $F_{X}$ is applied to a rib member in the mesh, the deflection at the tip of the rib along the Xdirection is induced by the cosine component of the force $F_{X}$ resulting in a total deflection given by:

$$
\delta_{\text {Total }}=\frac{F_{X} \operatorname{Cos}(\theta) l^{3}}{E_{0} h t^{3}}
$$


Where $E_{0}$ is the Young's modulus of the mesh's material, $h$ is the height of the mesh, $w$ is the width of the major ribs and $l$ is the length of the minor ribs.

A reference area for a single element of the mesh with the height $h$ and a length $s$ will be used to calculate an equivalent stress along the $X$ direction where $s$ is the separating distance along the chord-wise direction between each two elements in the accordion mesh.

$$
\begin{gathered}
\boldsymbol{\sigma}_{\boldsymbol{X}}=\frac{\boldsymbol{F}_{\boldsymbol{X}}}{\boldsymbol{h} \boldsymbol{s}} \\
\varepsilon_{X}=\frac{2 \delta_{\text {Total }} \cos (\theta)}{2 l \sin (\theta)+w}
\end{gathered}
$$

The equivalent in-plane Young's modulus for the mesh can be evaluated as:

$$
E_{e q}=\left(\frac{t}{l}\right)^{3} \frac{E_{0}(2 l \sin (\theta)+w)}{2 s \cos ^{2}(\theta)}
$$

Similarly, the equivalent shear modulus for the mesh is:

$$
G_{e q}=\left(\frac{t}{l}\right)^{3} \frac{E_{0}(2 l \sin (\theta)+w)}{2 s \sin ^{2}(\theta)}
$$

The main factors that affects the equivalent Young's and shear moduli of the mesh are found from equations (5.6) and (5.7) to be the thickness $t$, the length of the minor ribs $l$ and the angle $\theta$ between the major and minor ribs. $E_{e q}$ and $G_{e q}$ are directly proportional to $t^{3}$ and inversely proportional to $l^{3}$ so the behavior of the mesh could be easily tuned by changing the values of $t$ and $l$. The exact values of these parameters should be chosen in accordance with the maximum allowable out-of-plane deflection of the minor ribs given by:

$$
\delta_{Z \max }=\frac{F_{Z} l^{3}}{E_{0} t h^{3}}
$$


In this study, equation (5.8) was used to obtain the dimensions of the minor ribs to be $40 \mathrm{~mm}$ and $1 \mathrm{~mm}$ for the length $l$ and the thickness $t$ respectively. This will result in a maximum deflection of $\delta_{Z \text { max }} \approx 0.26 \mathrm{~mm}$ when the mesh is manufactured from a material with elastic modulus of $E_{0}=$ $2.3 \mathrm{GPa}$.

The angle between the minor and the major ribs appeared in both expressions (5.6) and (5.7) as sine and cosine functions. As a trade-off for $\theta$, an angle of $45^{\circ}$ is chosen to compromise the desired minimal values of both moduli.

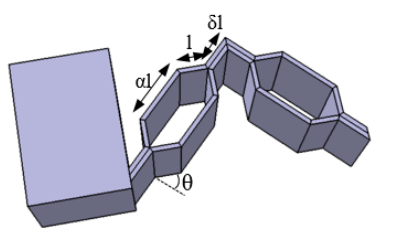

Central Hexagonal $L=(2 \delta+\alpha+2 * \operatorname{Sin}(\theta)) * l$

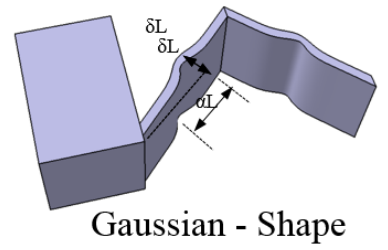

$y(x)=\delta * L * \frac{e^{\left.-\frac{2 \alpha-L}{\alpha * L}\right)^{2}}-e^{-\frac{1}{\alpha^{2}}}}{1-e^{-\frac{1}{\alpha^{2}}}}$

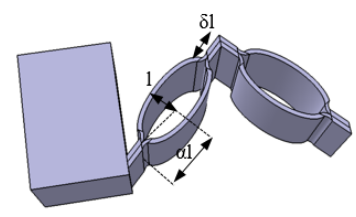

Central Ellipse

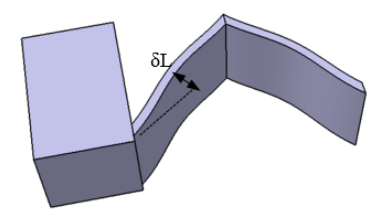

Cosine - Shape

$y(x)=\frac{\delta * L}{2} *\left(1-\operatorname{Cos}\left(2 * \pi \frac{x}{L}\right)\right)$

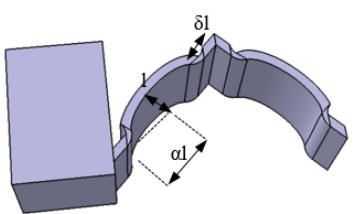

Central Half Ellipse

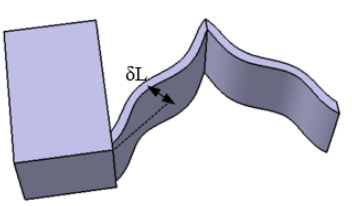

Sine - Shape

$y(x)=\frac{16}{3} * \delta * x *\left(1-\frac{x}{L}\right) * \operatorname{Sin}\left(2 \pi \frac{x}{L}\right)$

Figure 5.6. Different shapes of the minor ribs proposed by the author to reduce the local stresses for the in-plane motions.

The commercial code ANSYS Mechanical was used to perform a finite element analysis (FEA) for the in-plane motions of the mesh. This analysis showed excess local strain on the minor ribs, hence, more actuation force is required to morph the skin. In a trial to minimize these local strains, the shape of the minor ribs was modified. The proposed shapes are shown with their corresponding governing equations in figure (5.6).

These shapes were studied numerically to reduce the local stresses and strains. The span of the mesh in each case was changed by $12 \%$ and the sweep angle was changed to produce $30 \%$ global shear strain on the mesh. The maximum local strains obtained from each shape is shown in figure (5.7).

It was found that the cosine shaped ribs experienced the least local strain with a deduction of $12.94 \%$ compared to the mesh with straight ribs. This drop in the local strain is desired to minimize the actuation. 


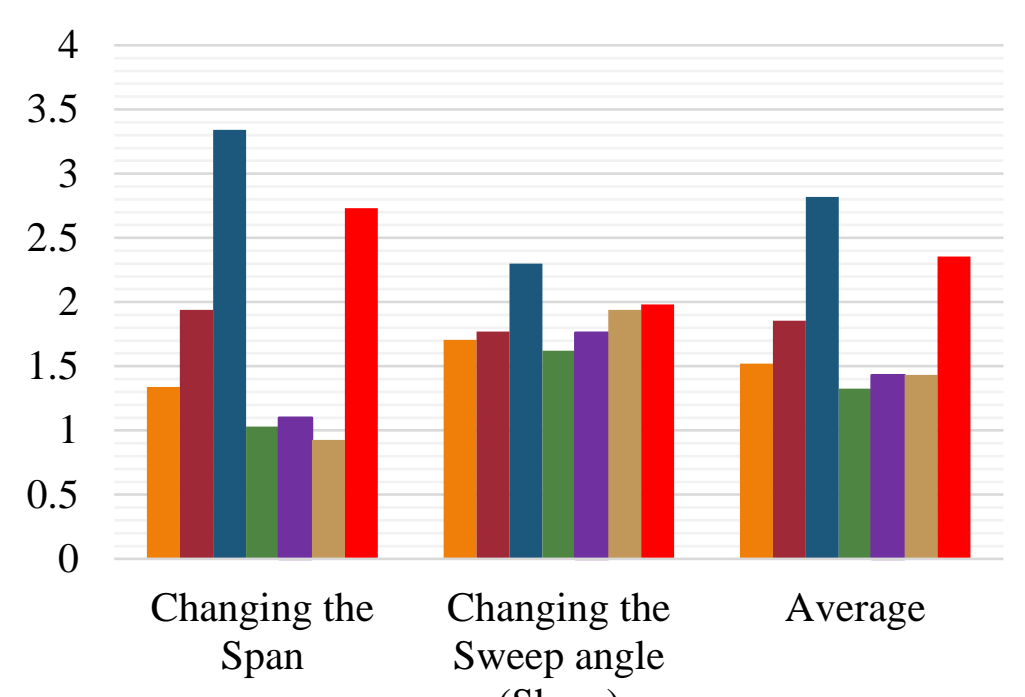

(Shear)

- Straight
Ribs
- Sine Shape
- Gaussian
Shape
Cosine
Shape
Central Half
Ellipse
Central
Hexagon
Central
Ellipse

Figure 5.7. Maximum local strain obtained from the FEA when different shapes for the minor ribs are used.

\section{Out-of-Plane Motion:}

The out-of-plane motions of the wing are encountered when changing the twist and the dihedral angles of the wing. The zero-Poisson ratio accordion mesh inherits a low bending stiffness along the direction perpendicular to the major ribs. This serves perfectly in favor of achieving the required dihedral angles, however, twisting the mesh introduces highly concentrated stresses near the joining edge of the minor ribs as shown in figure (5.8 a). These excess stresses are practically understood as more actuation forces and lower life time for the mesh before failure. The factors affecting the twist angle $\alpha$ of ribs with constant cross-sectional area are found in:

$$
\alpha=\frac{T l}{J G_{0}}
$$

Where $T$ is applied torque, $l$ is the length of the minor rib, $J$ is the moment of inertia and $G_{0}$ is the shear modulus of the mesh material. The moment of inertia $J$ for rectangular sections depends mainly on the ratio between the sides of the rectangular section. As the ratio between the sides increases, the moment of inertia $J$ increases leading to a smaller twist angle $\alpha$ for constant torque or more local stresses for constant twist angle. In a trial to reduce the stresses induced by the twist motion, the minor ribs are divided into four smaller ribs, the height of each rib is one forth the height of the original rib. The newly introduced ribs are distributed relative to each other so that the maximum out-of-plane deflection of the ribs is not changed. Using FEA, the newly designed ribs have reduced the maximum stresses by $20.28 \%$ and the locations of these stresses are greatly reduced as shown in figure (5.8 b) compared to figure (5.8 a). 

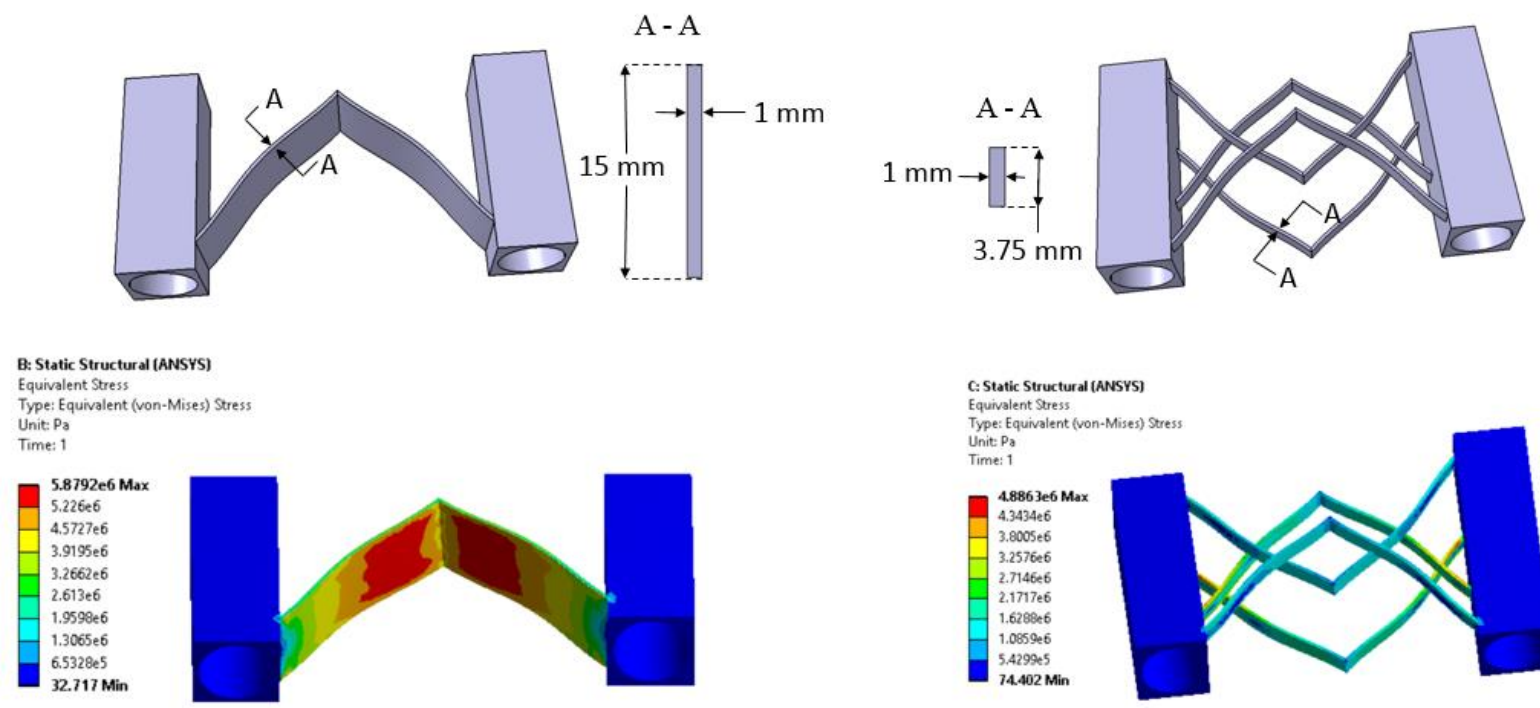

(a)

C: Static Structural (ANSYS) Equivalent Stress
Type: Equivalent (von-Mises) Stress Type: Equinalent (von-Mises) Stress
Unit: $\mathrm{Pa}$

Uime: 1

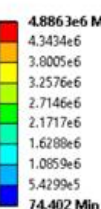

$74,402 \mathrm{Min}$

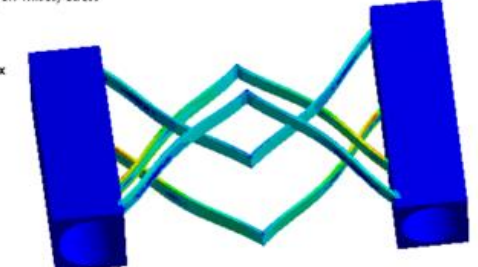

(b)

Figure 5.8. (a) Highly concentrated stresses when twisting a single minor rib compared to (b) low stresses when 4 minor ribs are used instead.

By doing this final modification, the mesh is now optimized to perform all the required morphing motions while needing minimum energy for actuation. The next section will focus on designing a sealant material that will cover the cellular structure to transfer the aerodynamic loads to the underlying mesh. 


\subsubsection{The Outer Composite Material}

The main challenge in the process of designing the outer layer of the morphing skin is to optimize the competing demands of a flexible continuous sealant material and still maintain its stiffness to carry all the aerodynamic loads. This can only be achieved by using a tailored composite material that has a flexible matrix material reinforced with longitudinal fibers along the wing's chord-wise direction. Figure (5.9), depicts the design of the required composite material.

The matrix material is the one which is in direct contact with the surrounding fluid, so it has to be chemically stable and maintain its flexibility over a wide temperature range. This made elastomers, a perfect candidate for this task where they are chemically inert and maintain their mechanical properties over a wide range of temperatures. Figure (5.10) shows different elastomer materials and their temperature range. From this figure, it can be observed that Silicone rubber maintain its properties over a wide range of positive and negative temeratures making it the best choice for the composite's matrix material.

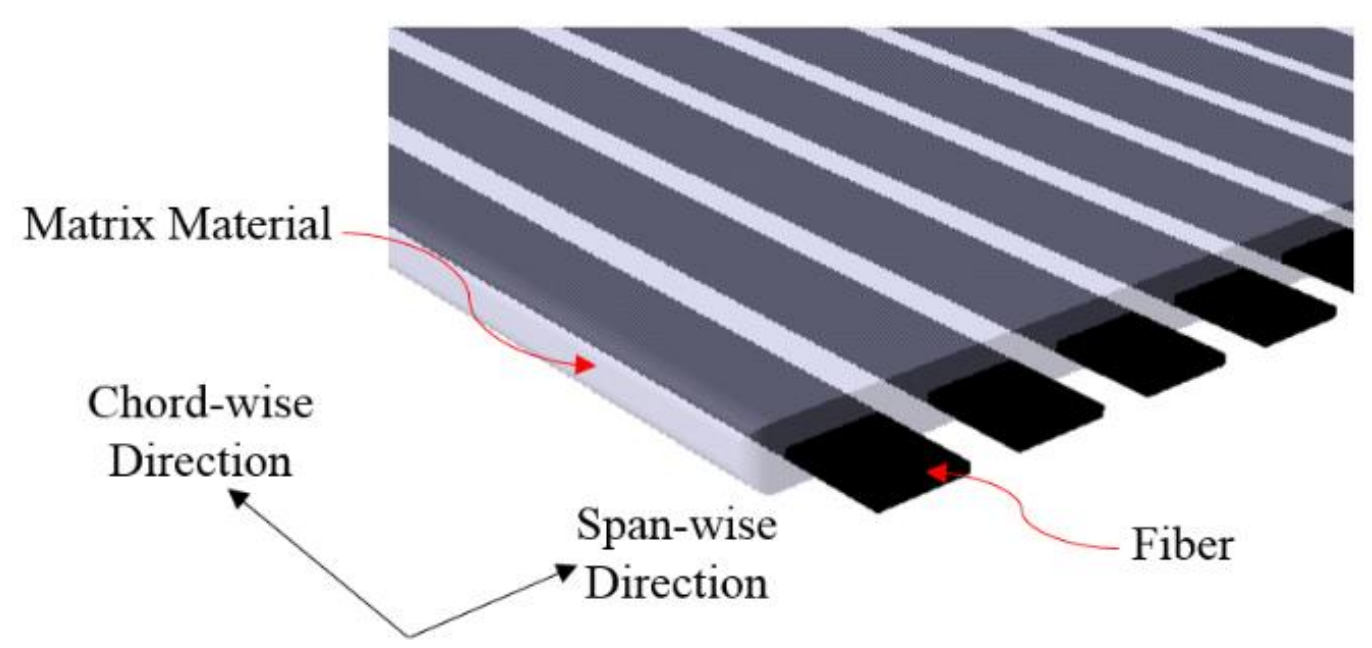

Figure 5.9. Design of the composite material with its fibers parallel to the chord-wise direction.

The shore hardness of the Silicone rubber has to be moderate, not too soft to prevent buckling and wrinkling, and not too hard to minimize the required actuation force. With these specifications, silicone rubber Ecoflex $\odot 0030$ was chosen as the matrix material composite. Ecoflex $\odot 0030$ has a Young's modulus of $29 \mathrm{kPa}$ and a Poisson ratio of 0.47 [86]. 


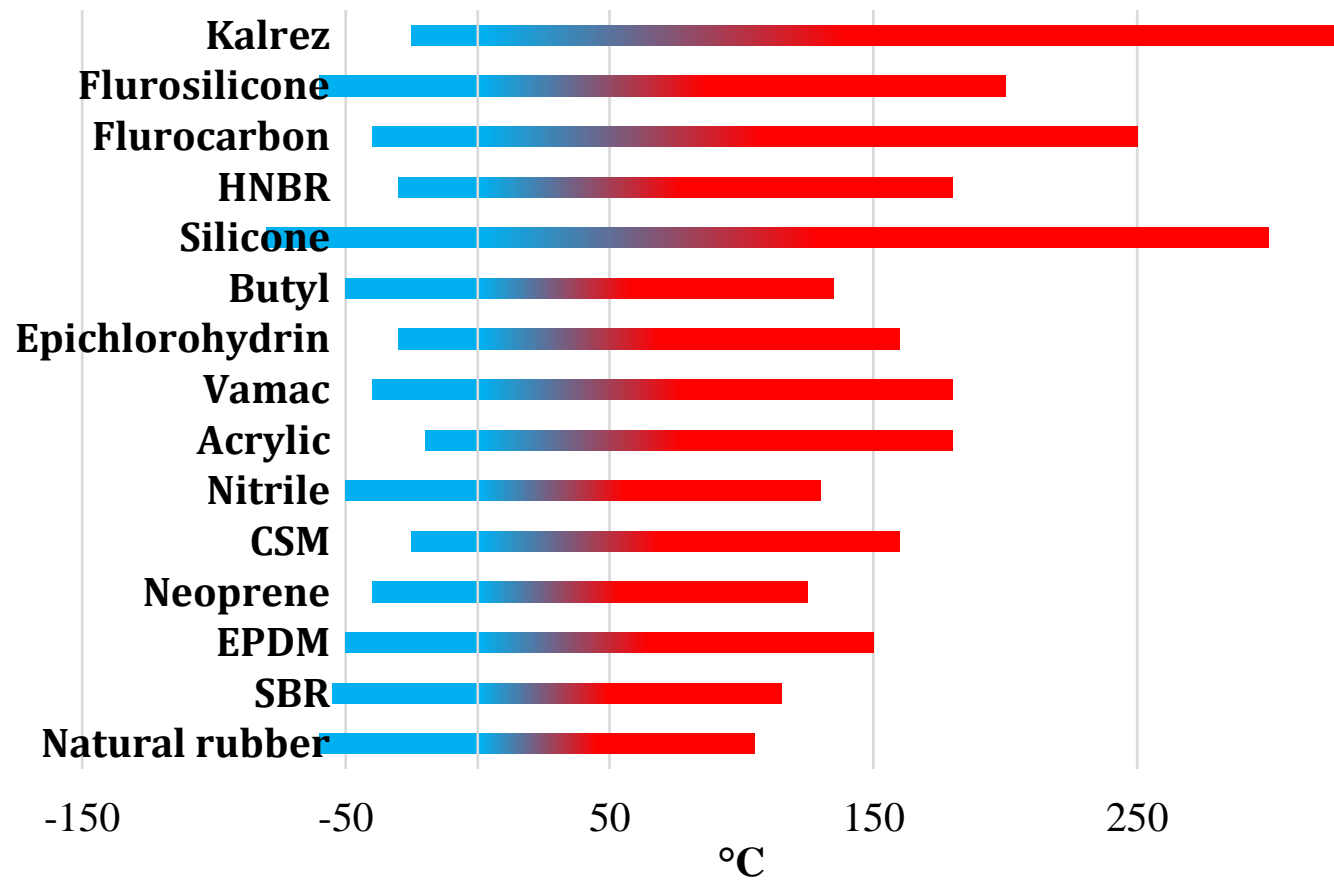

Figure 5.10. Temperature range of different elastomer materials.

In order to reinforce the composite material along the chord-wise direction, fibers with high strength have to be embedded inside the matrix material and maintain their direction parallel to the chord-wise direction. This direction will be the longitudinal one and characterized with a higher elastic modulus compared to the transverse span-wise direction which is perpendicular to the aligned fibers.

Carbon fibers are among the common materials used for reinforcing elastomers. They are characterized by their high specific strength (strength-to-weight ratio) that is nearly ten times the specific strength of stainless steel. In this study, unidirectional carbon fibers with a Young's modulus of $181 \mathrm{GPa}$ are embedded in the composite material along the longitudinal direction. However, due to the nature of carbon fibers, they can only withstand tensile loads and cannot bear any compressive loads. For that reason, thin aluminum strips will be also tested as reinforcement fibers for the composite materials.

Four different composite samples were manufactured to test the out-of-plane deflection of the skin when attached to the cellular substructure. The first two used carbon fibers with a fiber volume fraction of $2.5 \%$ and $5 \%$. The other two samples will be reinforced with aluminum strips in percentages of $3 \%$ and $4 \%$.

Table (5.1) shows the highly anisotropic properties of the tailored composite material which are estimated using the rule of mixture for composite. 
Table 5.1. Properties of the four composite samples estimated using the rule of mixture for composites.

\begin{tabular}{ccccc}
\hline Case & $\mathbf{1}$ & $\mathbf{2}$ & $\mathbf{3}$ & $\mathbf{4}$ \\
& Carbon Fibers & Carbon Fibers & Aluminum Strips & Aluminum Strips \\
\hline $\boldsymbol{V}_{\boldsymbol{f}}$ & $2.5 \%$ & $5 \%$ & $3 \%$ & $4 \%$ \\
\hline $\boldsymbol{E}_{\text {Longitudinal }}$ & $5.5 \mathrm{GPa}$ & $11 \mathrm{GPa}$ & $2.07 \mathrm{GPa}$ & $2.76 \mathrm{GPa}$ \\
\hline $\boldsymbol{E}_{\text {Transverse }}$ & $29.744 \mathrm{kPa}$ & $30.526 \mathrm{kPa}$ & $28.130-29.897$ & $27.840-30.208$ \\
& & & $\mathrm{kPa}$ & $\mathrm{kPa}$ \\
\hline $\boldsymbol{v}_{\text {Princioal }}$ & 0.4633 & 0.4565 & 0.4659 & 0.4646 \\
\hline $\boldsymbol{v}_{\text {Seondary }}$ & $2.505 \mathrm{e}-6$ & $1.266 \mathrm{e}-6$ & $6.729 \mathrm{e}-6$ & $5.084 \mathrm{e}-6$ \\
\hline
\end{tabular}

It can be observed from table (5.1) that the composites with the carbon fibers have a higher longitudinal Young's modulus because of their original high tensile strength. During the manufacturing process, the aluminum strips were observed to have a weak bonding to the silicone rubber, for that reason the actual transverse Young's modulus will be slightly lower than that provided by the rule of mixture. 


\subsubsection{Prototyping and Out-of-Plane Testing}

For quick prototyping, the 3D printing technology is used to convert the CAD design of the cellular structure into a prototype using Acrylonitrile butadiene styrene (ABS) plastic with a Young's modulus of $E_{0}=2.3 \mathrm{GPa}$ and a Poisson ratio of $v_{0}=0.35$. Figure (5.11) shows the 3D printed prototype of the cellular mesh when morphed in the four required motions, namely, tension/compression, changing the sweep angle, changing the twist angle and changing the dihedral angle.

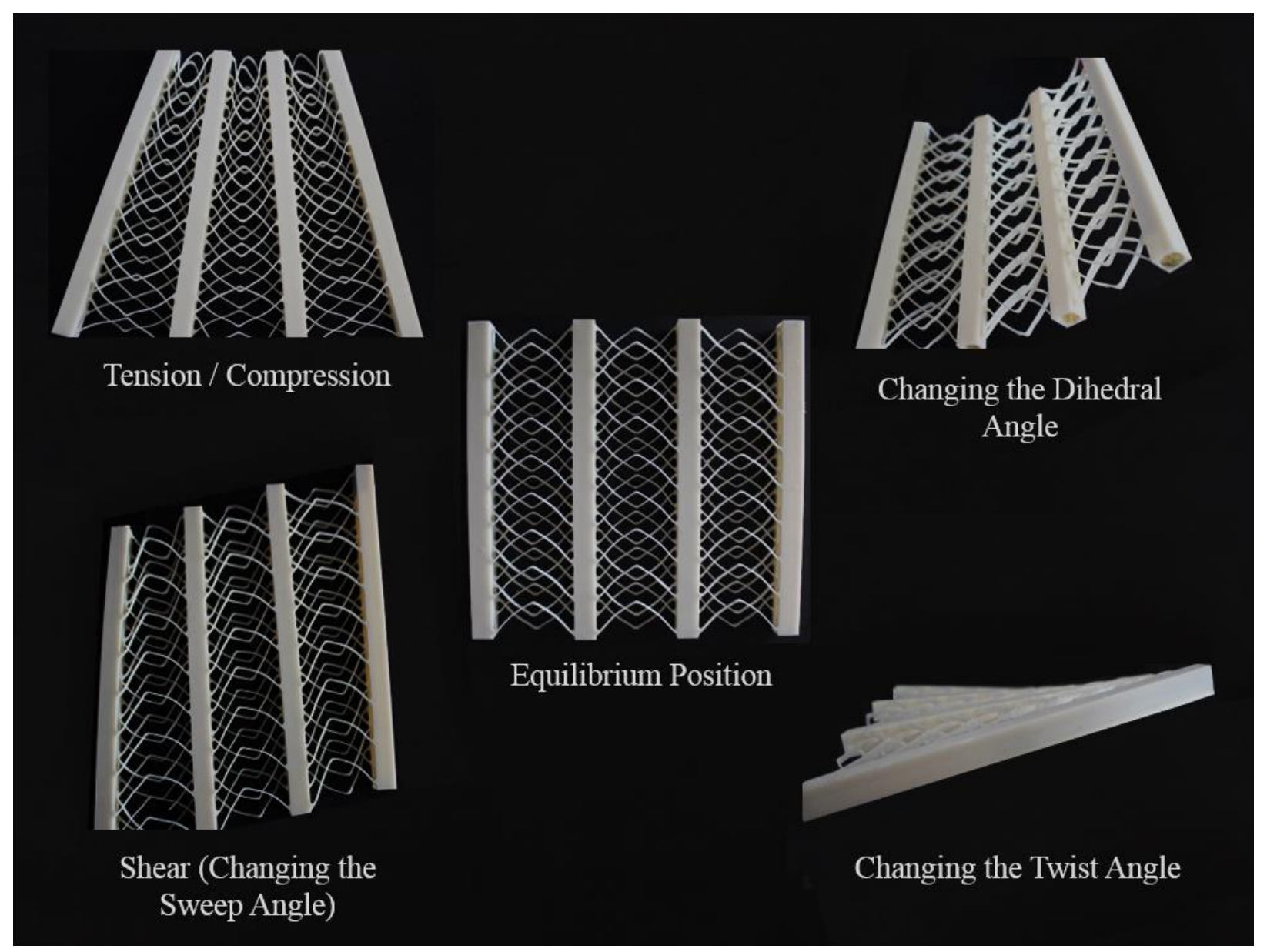

Figure 5.11. Prototype of the morphing cellular structure when morphed in the allowable degrees of freedom.

The four different outer layer samples were integrated with the reinforced cellular substructure using a silicone-based adhesive. The adhesive has thermal and mechanical properties similar to the used Ecoflex $\odot 0030$. This insures a homogeneous bonding and avoids any degradation in the desired properties. 


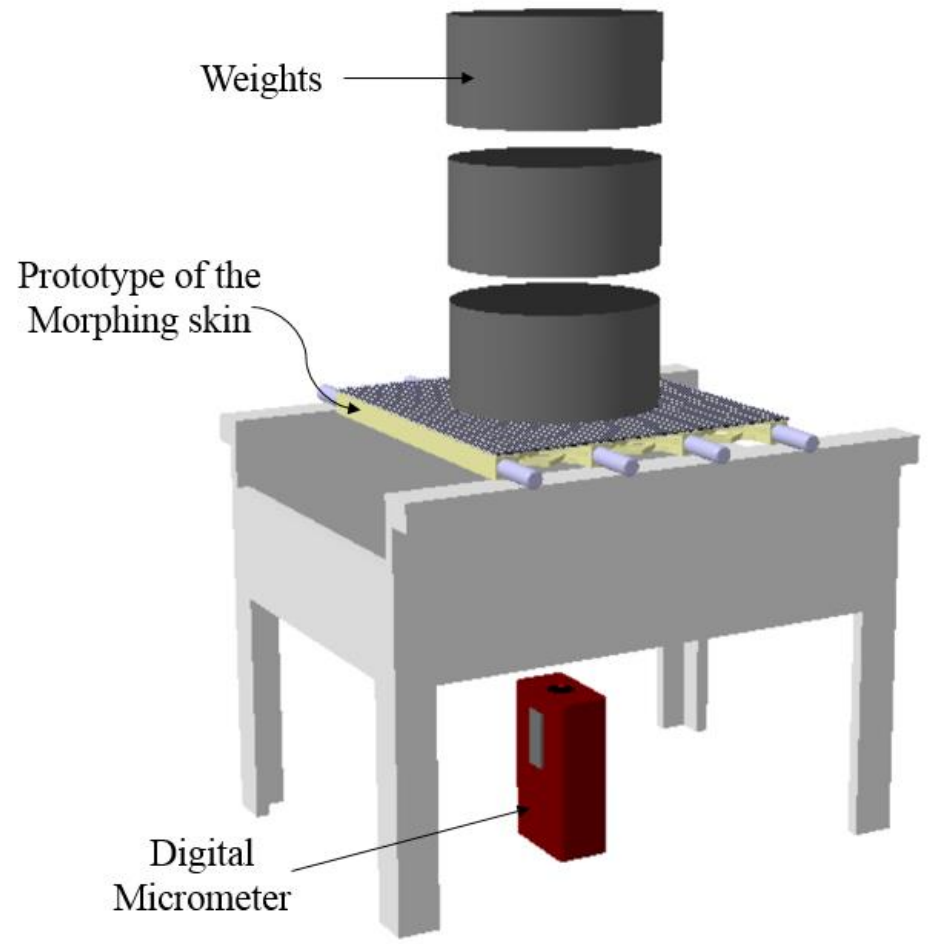

Figure 5.12. Experimental set-up to test the out-of-plane deflection of the morphing skin.

To test the out-of-plane deflection of the integrated skin, normal loads were applied to the skin prototype resembling the aerodynamic pressure. The experimental setup shown in figure (5.12) is designed to support the aluminum rods from both sides like the spars. Below the setup is a digital micrometer which comes in contact with the lower surface of the composite material.

Table 5.2. Results of the out-of-plane deflection test.

\begin{tabular}{c||cccc}
\hline $\begin{array}{c}\text { Deflection of } \\
\text { the skin at: }\end{array}$ & $\begin{array}{c}\text { Case 1 } \\
\text { Carbon Fibers }\end{array}$ & $\begin{array}{c}\text { Case 2 } \\
\text { Carbon Fibers }\end{array}$ & $\begin{array}{c}\text { Case 3 } \\
\text { Aluminum Strips }\end{array}$ & $\begin{array}{c}\text { Case 4 } \\
\text { Aluminum Strips }\end{array}$ \\
\hline $\begin{array}{c}\mathbf{2 . 5} \% \\
\text { 10\% }\end{array}$ & 1.89 & $\mathbf{5 \%}$ & $\mathbf{3} \%$ & $\mathbf{4 \%}$ \\
\hline $\begin{array}{c}\text { Normal } \\
\text { Position }\end{array}$ & 1.94 & 1.91 & 2.86 & $2.99 \mathrm{~mm}$ \\
\hline $10 \%$ Extension & 1.66 & 1.77 & 2.59 & $2.88 \mathrm{~mm}$ \\
\hline
\end{tabular}

On top of the integrated morphing skin, loads were added in six successive increments of $48 \mathrm{~N}$ covering a circular area of $0.01767 \mathrm{~m}^{2}$ resulting in a maximum pressure of $16.32 \mathrm{kPa}$. Each of the 
four cases was tested in three different positions; equilibrium, 10\% compression and $10 \%$ extension. The results of the test are listed in table (5.2).

The results in table (5.2) show that composite skins with carbon fibers have experienced less outof-plane deflection compared to the aluminum strips. This is due to the higher longitudinal elastic moduli of carbon fibers compared to aluminum. 


\subsection{Aerodynamics of Flexible Morphing Skin}

\subsubsection{FEA for Wrinkle Profile Prediction:}

To qualitatively predict the shape of the wrinkles forming on the surface of the flexible morphing skin, a finite element analysis (FEA) was performed. A single module of the morphing wing changes its geometry over the range of morphing. The twist motion was observed to introduce the largest magnitude of wrinkles when reaching its limits of $\pm 5^{\circ}$. Figure (5.13) shows the flexible morphing wing model twisted by $5^{\circ}$ continuously distributed along the span of 1 meter. The colors shown in figure (5.13) represents the magnitude of absolute displacement obtained from the finite element model.

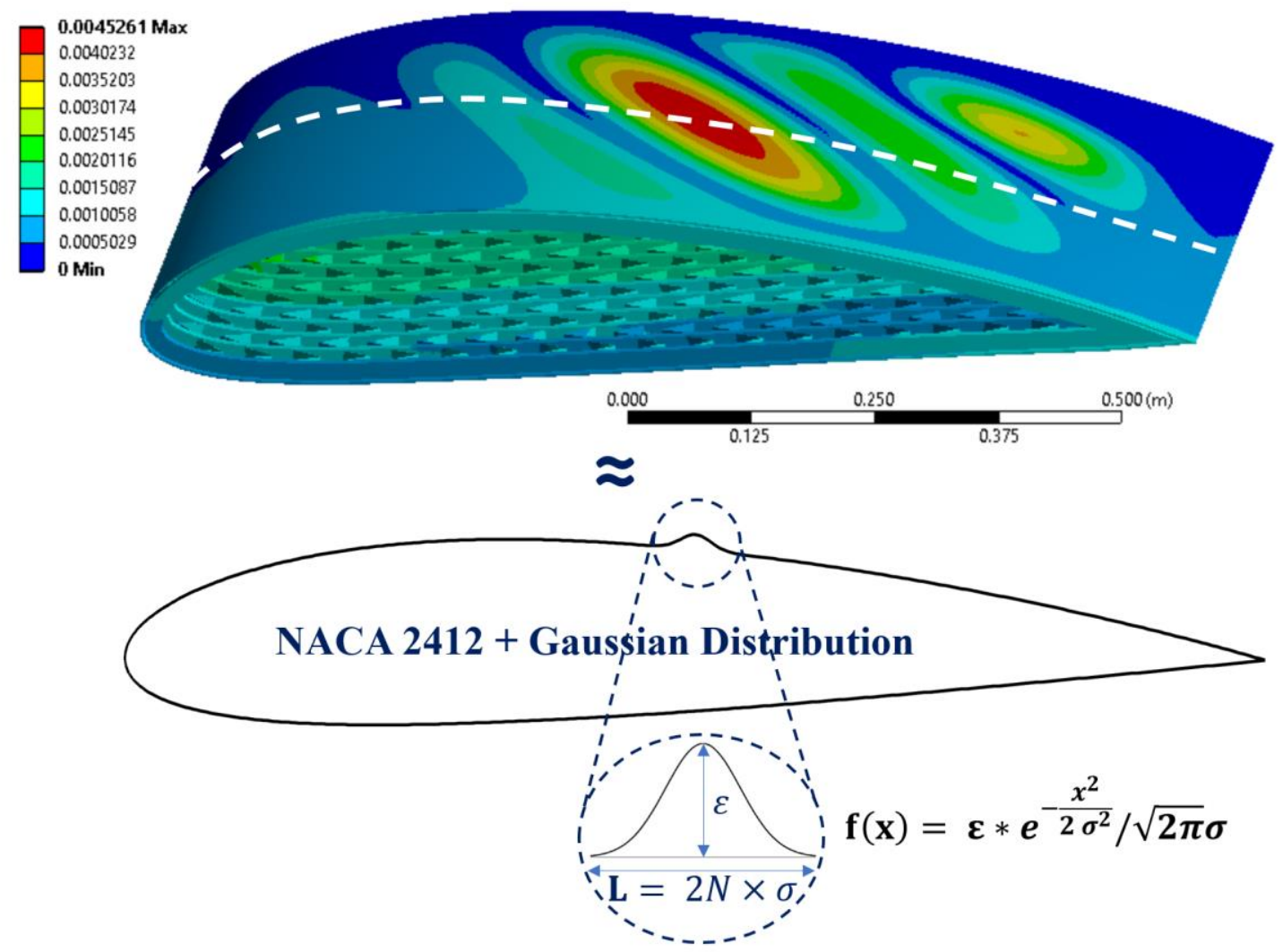

Figure 5.13. Interpolating the skin wrinkles into Gaussian distribution and adding it to the NACA 2412 profile.

A 2D profile of the morphed wing was extracted from the finite element model at the location of the maximum deformation. Since the purpose of this study is to systematically examine the aerodynamic effects of wrinkles on morphing skin, the shape of these formed wrinkles has to be 
mathematically articulated. Different known mathematical functions were tested, and the simple Gaussian distribution function showed the best agreement with the profile extracted from the finite element model. The Gaussian distribution function is given by equation (5.10):

$$
f(x)=\varepsilon e^{-\frac{x^{2}}{2 \sigma^{2}}}
$$

Where $\varepsilon$ is the height of the Gaussian distribution, $\sigma$ is the standard deviation and is equal to 1 in case of a simple Gaussian distribution. The function has a zero mean value and is symmetric about $\mathrm{x}=0$. The $1 / 2$ factor used with the exponent ensures a unit variance. This simple Gaussian distribution is added to the NACA 2412 profile as shown in figure (5.14).

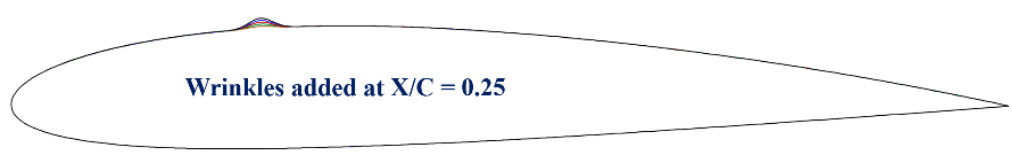

(a)

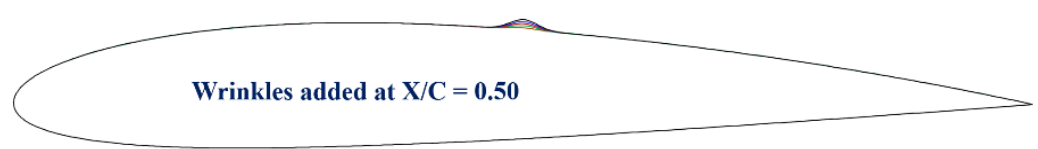

(b)

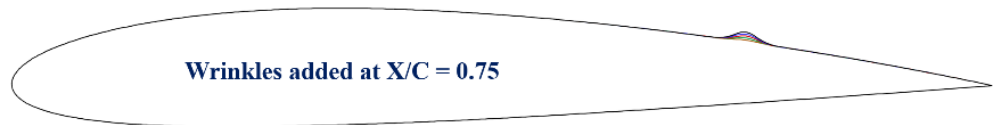

(c)

Figure 5.14. 15 configurations of the wrinkled NACA 2412 airfoil with wrinkles added at (a)

$$
\mathrm{X} / \mathrm{C}=0.25 \text {, (b) } \mathrm{X} / \mathrm{C}=0.50 \text {, and (c) } \mathrm{X} / \mathrm{C}=0.75 \text {. }
$$

The FEA showed that the winkles on the wing are usually diagonally oriented along the span of the wing as shown in figure (5.13). This means that at different sections along the wing's span, the wrinkle location is shifted along the chord-wise direction. For that reason, the Gaussian distribution is imposed on the NACA 2412 profile at three different locations along the chord-wise direction. These locations are at $\mathrm{X} / \mathrm{C}=0.25, \mathrm{X} / \mathrm{C}=0.5$ and $\mathrm{X} / \mathrm{C}=0.75$, where $\mathrm{C}$ is the chord length of the airfoil. At each of these locations, five different heights of Gaussian distributions were added 
to the NACA 2412 profile ranging from $\varepsilon / \mathrm{C}=0.002$ to $\varepsilon / \mathrm{C}=0.01$ resulting in 15 different configurations of the airfoil as shown in figure (5.14).

The 15 different configurations or wrinkled airfoils shown in figure (5.14) cover a wide range of wrinkles' profiles at different locations along the chord-wise direction. This gives a good representation of the diagonally oriented wrinkles shown in figure (5.13).

\subsubsection{Modeling and Accuracy Assessment:}

\section{Boundary conditions and Baseline Geometry:}

This study focuses on the performance of wrinkled airfoils at the incompressible flow regime and low Reynolds number of 1 million. This flight regime is a common regime for unmanned aerial vehicles (UAV's) which is the main application of the immature morphing technology. The Mach number is set to 0.2 which corresponds to a mainstream velocity of $69.42 \mathrm{~m} / \mathrm{s}$ at a temperature of $300 \mathrm{~K}$. The NACA 2412 airfoil used has a unity chord length, a sharp trailing edge, and an angle of attack of $0^{\circ}$. The computational domain is wrapped around the airfoil in a C-topology where the blocks are distributed along the computational domain in a $\mathrm{C}$ shape. The computational domain extends to 32 chords away from the airfoil and the boundary conditions at the inlet and outlet of the computational domain are set to pressure-far-field. The pressure far-field prevents pressure waves from being reflected at the boundaries by applying Riemann invariants for a onedimensional flow normal to the boundary.

\section{Accuracy and Spatial Convergence:}

One of the demanding tasks in any CFD study is determining the optimal density of the computational grid that can capture all the important flow features, and at the same time consumes reasonable computational time. To reach this demanding balance, the Richardson's extrapolation method explained in chapter 2 is used on a family of three consecutively refined meshes as shown in figure (5.15). 

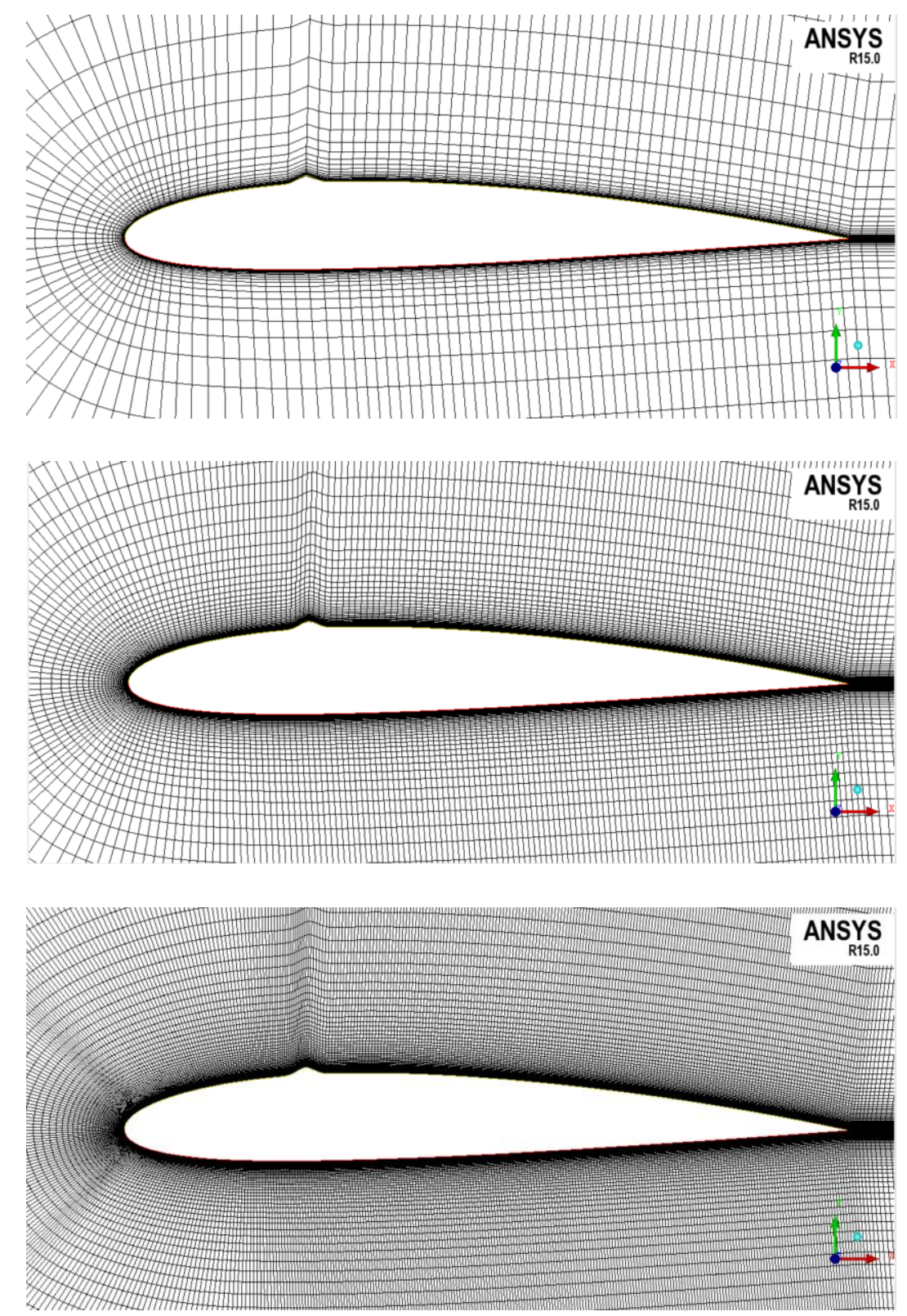

Figure 5.15. A family of three consecutively refined meshes used to apply Richardson's extrapolation method and estimate the spatial convergence.

The number of cells of the grids shown in figure (5.15) is approximately 9700, 38800 and 155200 cells for the coarse, medium and fine meshes, respectively. The lift coefficient $C l$ is used as the aerodynamic property $\mathcal{F}$ in equations (2.1) and (2.2). The observed order of accuracy $p$ calculated from equation (2.2) is found to be 2.0092 which is in a very good agreement with the theoretical $2^{\text {nd }}$ order accuracy used in the numerical solver. Equation (2.1) obtained a continuum value of 0.15739 , while the coarse, medium and fine meshes obtained lifting coefficients of 0.02811 , 0.12350 , and 0.14850 , respectively as shown in figure (5.16). 


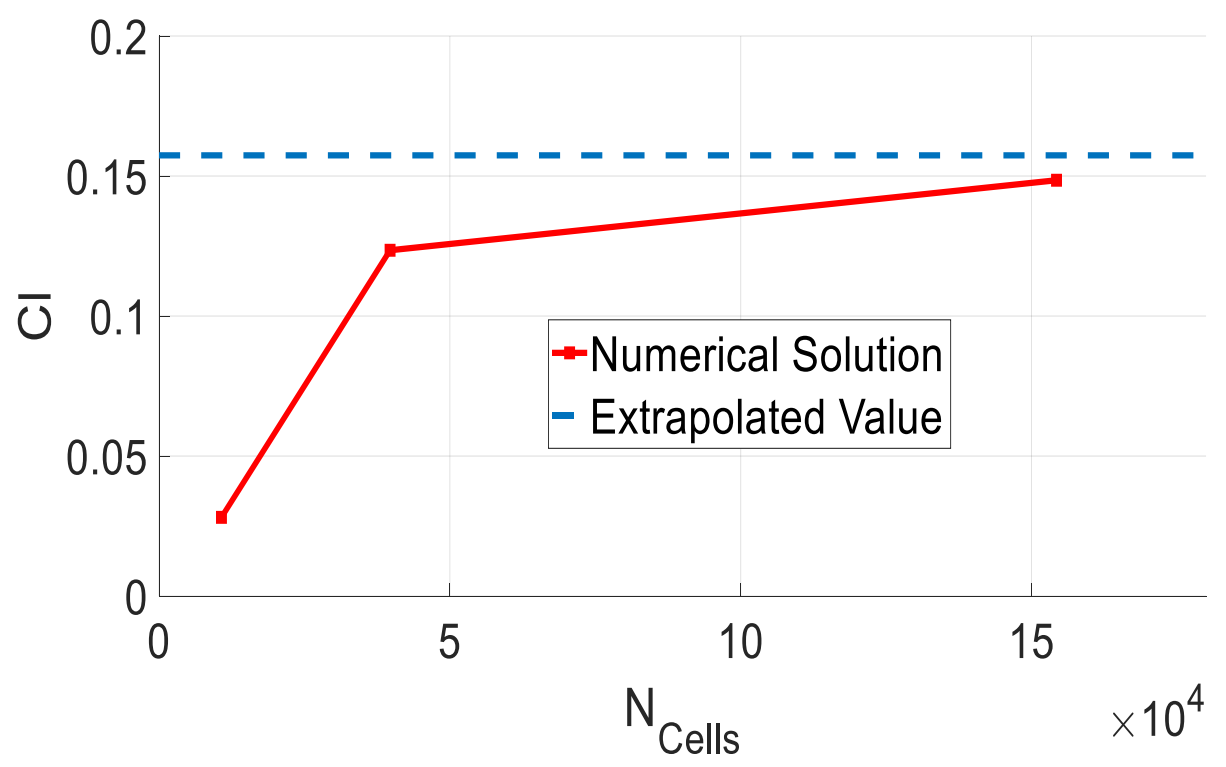

Figure 5.16. The lift coefficient values $\mathbf{C l}$ obtained on the coarse, medium and fine meshes, as well as the value extrapolated using Richardson's extrapolation method.

The percentage error of the fine mesh is found to be $0.88861 \%$. This means that the fine mesh can resolve the flow with high fidelity, and any extra refinement of the grid will be perceived as unnecessary computational expenses and will not add much to the accuracy of the solution. For that reason, all the results shown in this paper are obtained using grids with similar densities as the fine mesh shown in figure (5.16).

\subsubsection{Results and Discussion:}

Using the settings and the mesh presented in the previous section, the flow over the 15 configurations shown in figure (5.14) was solved. The main focus of this study is to determine the effect of the wrinkle height and location on the aerodynamic properties of the NACA 2412 airfoil. Figure (5.17) shows the lift coefficient $\mathrm{Cl}$ of the 15 configurations plotted using three curves, one for each wrinkle location. 


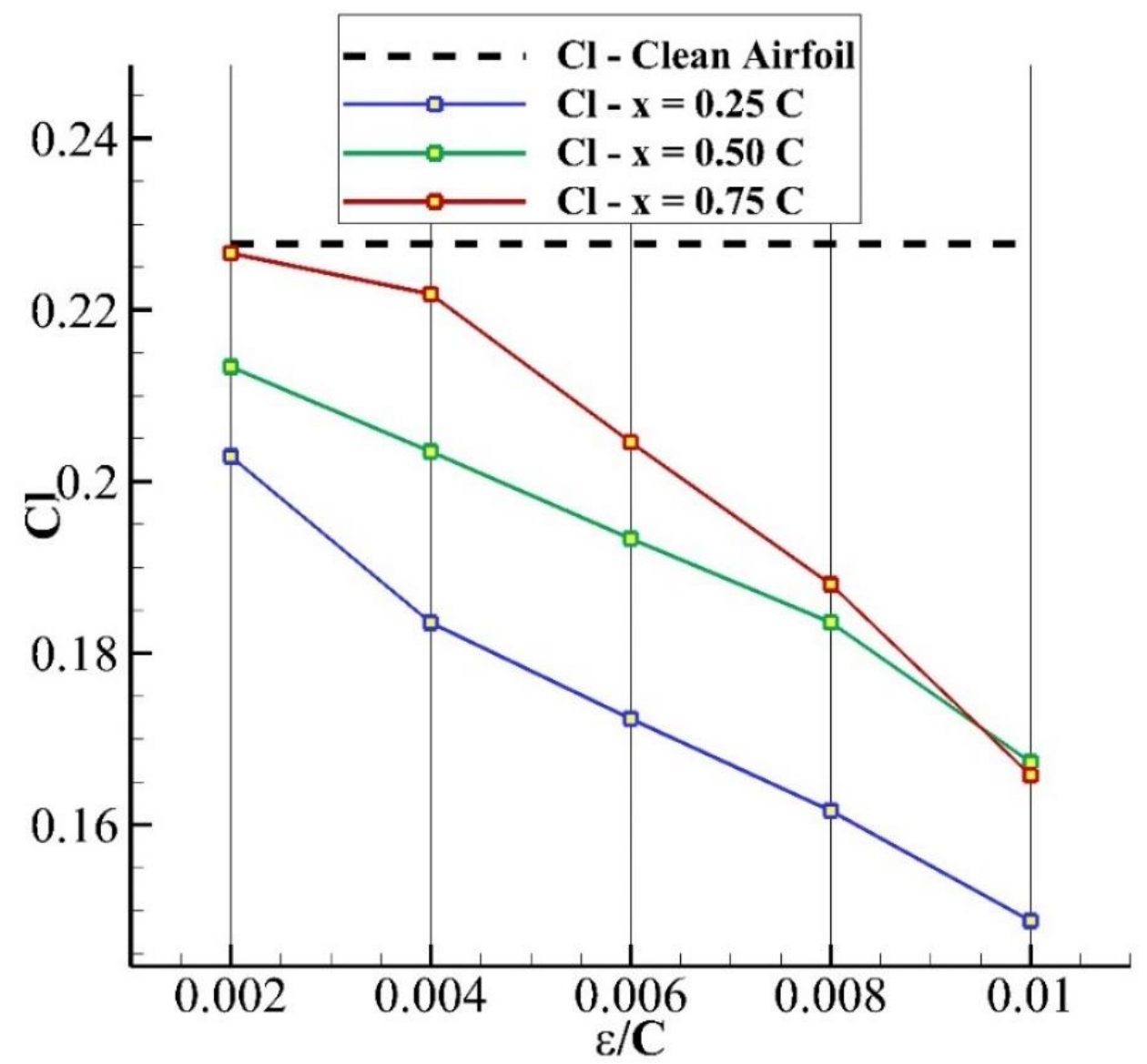

Figure 5.17. Lift coefficient values $\mathbf{C l}$ of wrinkled airfoils with different wrinkle heights and locations along the chord-wise direction of the airfoil.

Regardless of the wrinkle location, it is observed that the values of the lift coefficient have an inverse relationship with the wrinkle height. As the wrinkle height increases from $\varepsilon=0.002 \mathrm{C}$ to $0.01 \mathrm{C}$, the lift coefficient value $\mathrm{Cl}$ significantly dropped in a linear-like behavior. In case of a wrinkle located at $\mathrm{X}=0.25 \mathrm{C}$ and has a height of $\varepsilon=0.002 \mathrm{C}$, the lift coefficient value is $89.1 \%$ of the value of the clean airfoil. This value drops to $65.3 \%$ when the wrinkle height increases to $\varepsilon$ $=0.01 \mathrm{C}$ with a drop of approximately $5 \%$ in the lifting capabilities with every $0.002 \mathrm{C}$ increase in the wrinkle height. Similar results are observed for cases with the wrinkle located at mid chord length $(\mathrm{X}=0.5 \mathrm{C})$. The lifting capabilities of the airfoil dropped from $93.7 \%$ to $73.4 \%$ as the wrinkle height increased from $\varepsilon=0.002 \mathrm{C}$ to $\varepsilon=0.01 \mathrm{C}$, with an approximate increase of $4 \%$ for every $0.002 \mathrm{C}$ increase in the wrinkle height. Similarly, cases with a wrinkle located at $\mathrm{X}=0.75$ $\mathrm{C}$, the inverse relationship between the lift coefficient and the wrinkle height is sustained. The interesting observation in the third data set when the wrinkle is located at $\mathrm{X}=0.75 \mathrm{C}$, is that at a wrinkle height of $\varepsilon=0.002 \mathrm{C}$, the lift coefficient value is very close to the case of a clean airfoil. To understand the relationship between the lifting capabilities of the wrinkled airfoil and the 
wrinkle height, the pressure coefficient distribution over the upper surface of the airfoil is plotted in figure (5.18).

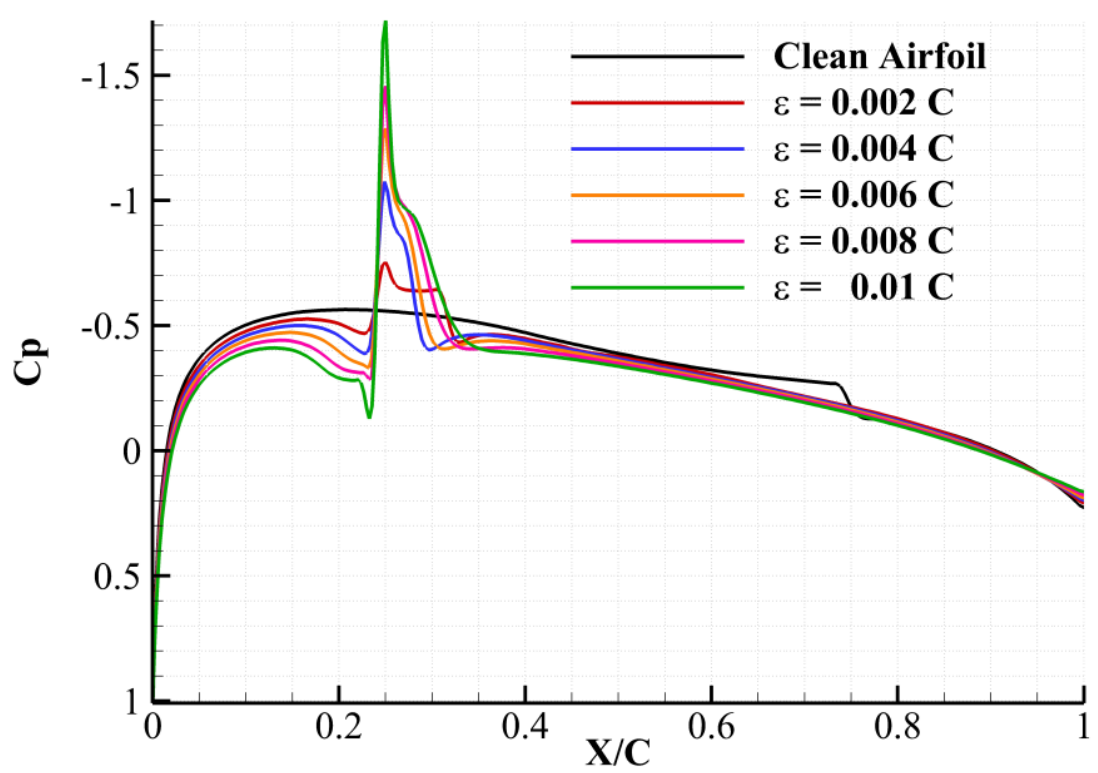

(a)

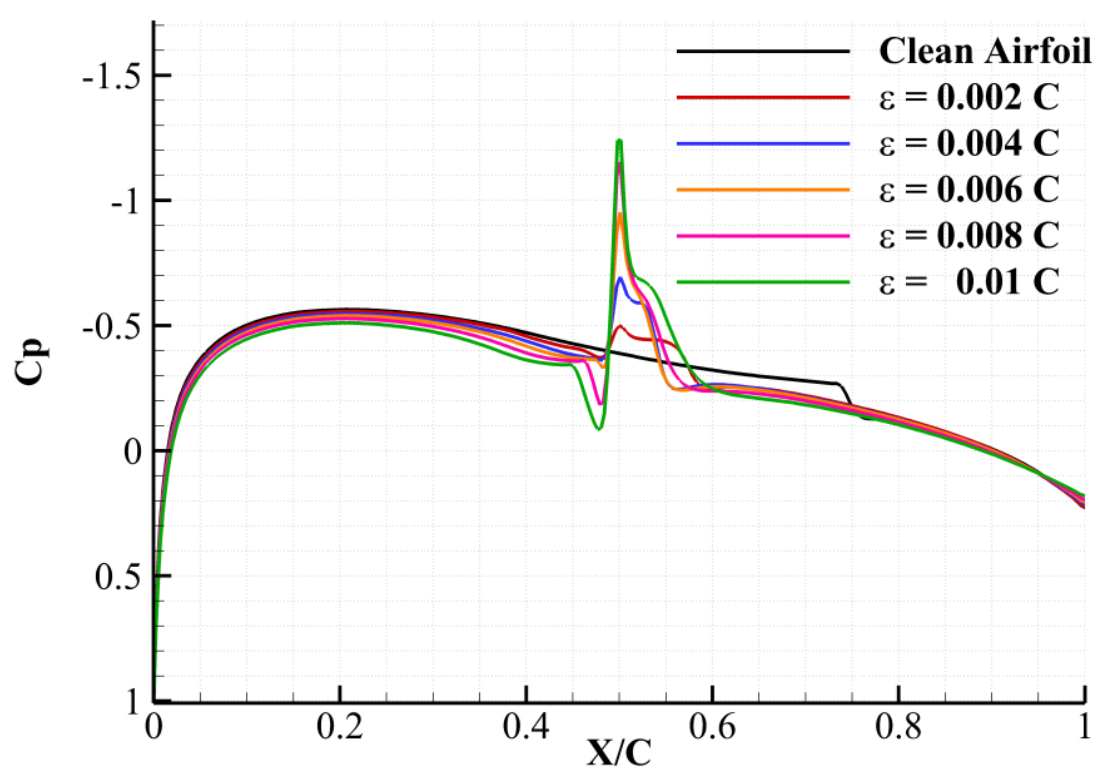

(b) 


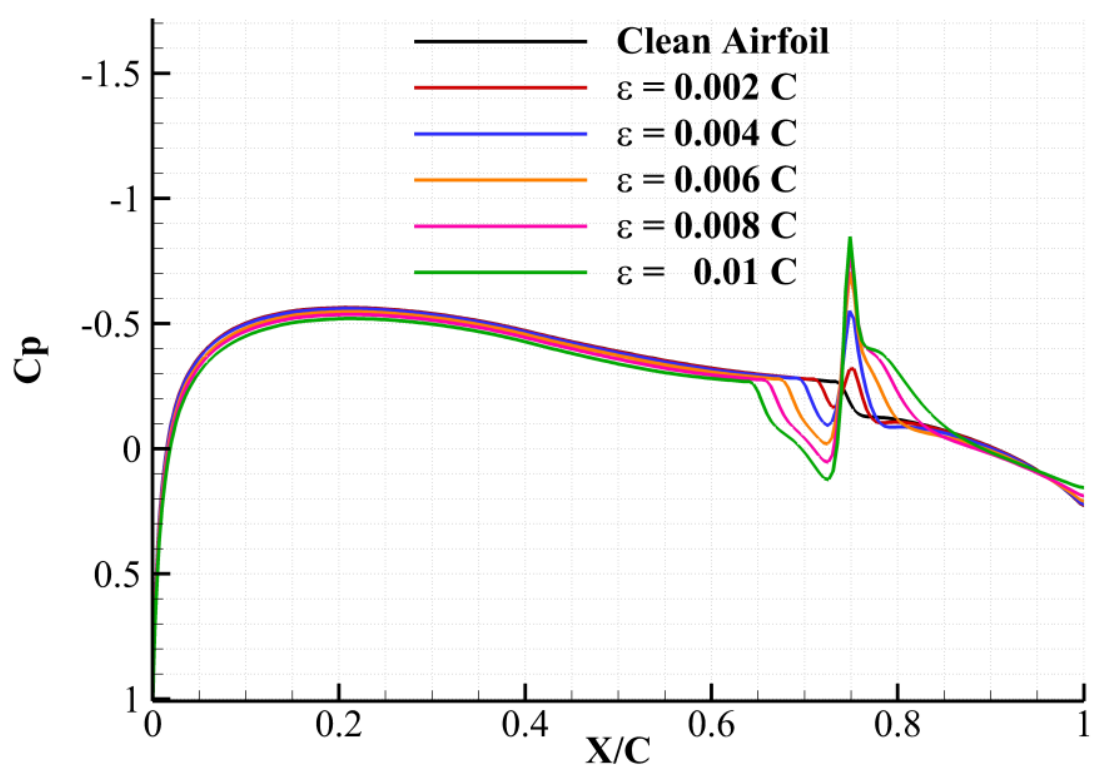

(c)

Figure 5.18. The pressure coefficient distribution for different wrinkles heights located at (a)

$$
\mathrm{X}=0.25 \mathrm{C} \text {, (b) } \mathrm{X}=0.50 \mathrm{C} \text { and (c) } \mathrm{X}=0.75 \mathrm{C} \text {. }
$$

Figure (5.18) shows that due to the flow acceleration over the wrinkle, the pressure locally decreases due to the conservation of momentum. On the other hand, inserting a wrinkle on the airfoil's surface obstructs the flow on the airfoil's surface causing an overall deceleration. This deceleration increases the pressure on the suction side of the airfoil, and consequently decreases the lifting capabilities of the airfoil. As the wrinkle height increases, the deceleration effect of the wrinkle becomes more prominent and results in higher static pressure on the suction side of the airfoil, and consequently smaller values for the lift coefficient. However, as the wrinkle location shifts towards the airfoil's trailing edge, the disturbance caused by introducing the wrinkle fades. Figure $(5.18 \mathrm{c})$ shows that in the case of a wrinkle height of $\varepsilon=0.002 \mathrm{C}$, the pressure distribution overlays the pressure distribution of the clean airfoil. For that reason, the lift coefficient of this case is very close to the value of the clean airfoil.

A similar analysis was conducted to establish the effect of installing the wrinkle on the airfoil's drag coefficient $\mathrm{Cd}$. Figure (5.19) shows the relationship between the wrinkle height and the drag coefficient $\mathrm{Cd}$ at three different wrinkle locations along the chord-wise direction of the airfoil. 


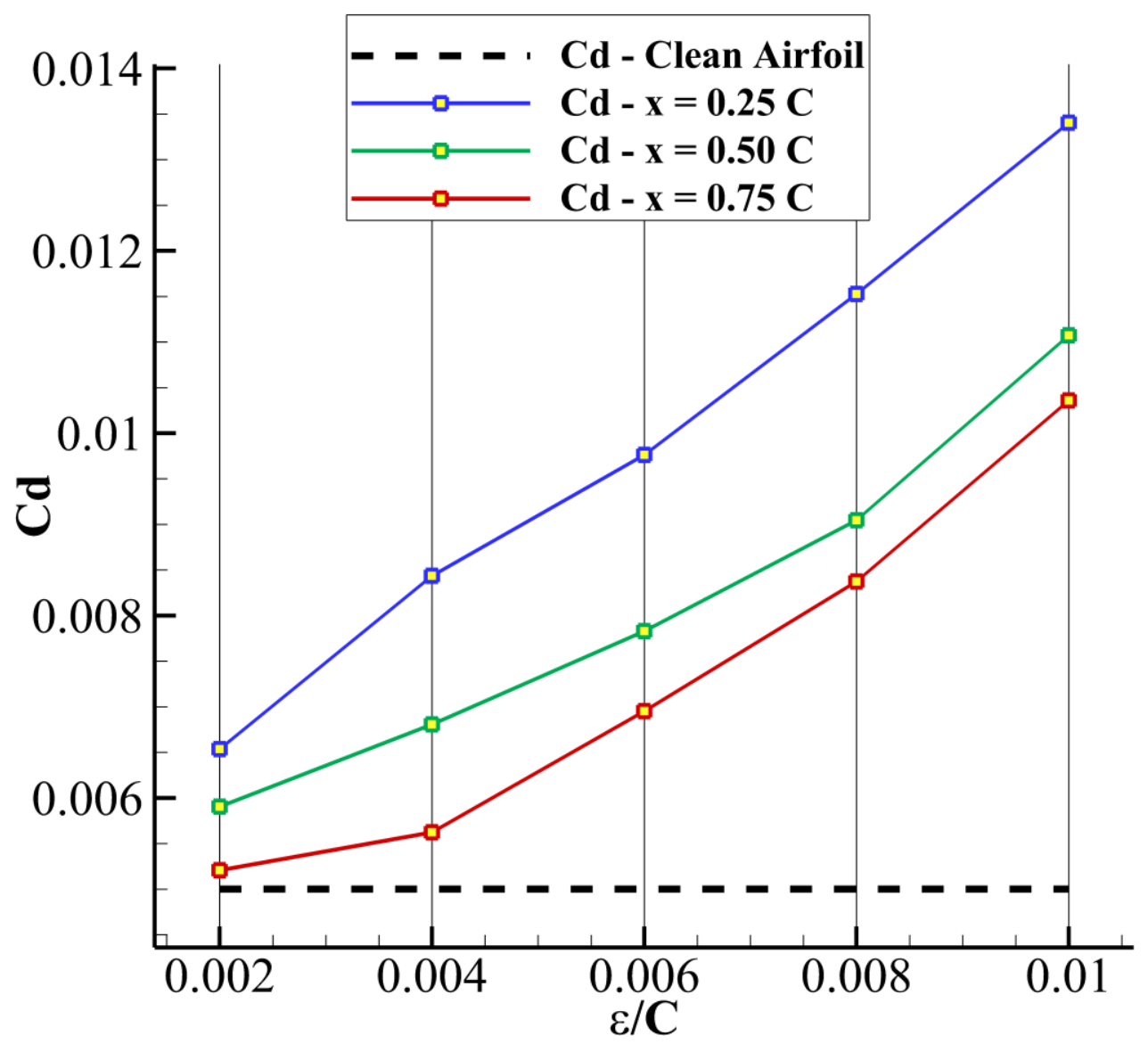

Figure 5.19. Drag coefficient values $C d$ of wrinkled airfoils with different wrinkle heights and locations along the chord-wise direction of the airfoil.

For the three wrinkle locations, $\mathrm{X}=0.25 \mathrm{C}, \mathrm{X}=0.50 \mathrm{C}$ and $\mathrm{X}=0.75 \mathrm{C}$, the drag coefficient increased linearly with the increase of the wrinkle height. For cases with a wrinkle located at $\mathrm{X}=$ $0.25 \mathrm{C}$, the drag coefficient increased from $130.7 \%$ to $267.9 \%$ of the drag value of the clean airfoil as the wrinkle height increased from $\varepsilon=0.002 \mathrm{C}$ to $\varepsilon=0.01 \mathrm{C}$. For cases with a wrinkle located at $\mathrm{X}=0.50 \mathrm{C}$, the drag coefficient value increased from $118.0 \%$ to $221.4 \%$, and for cases with a wrinkle located at $\mathrm{X}=0.75 \mathrm{C}$, the drag coefficient increased from $104.1 \%$ to $207.1 \%$. The reason behind this significant increase in the drag values can be explained from figure (5.20) which shows the skin friction coefficient distribution over the airfoil. 


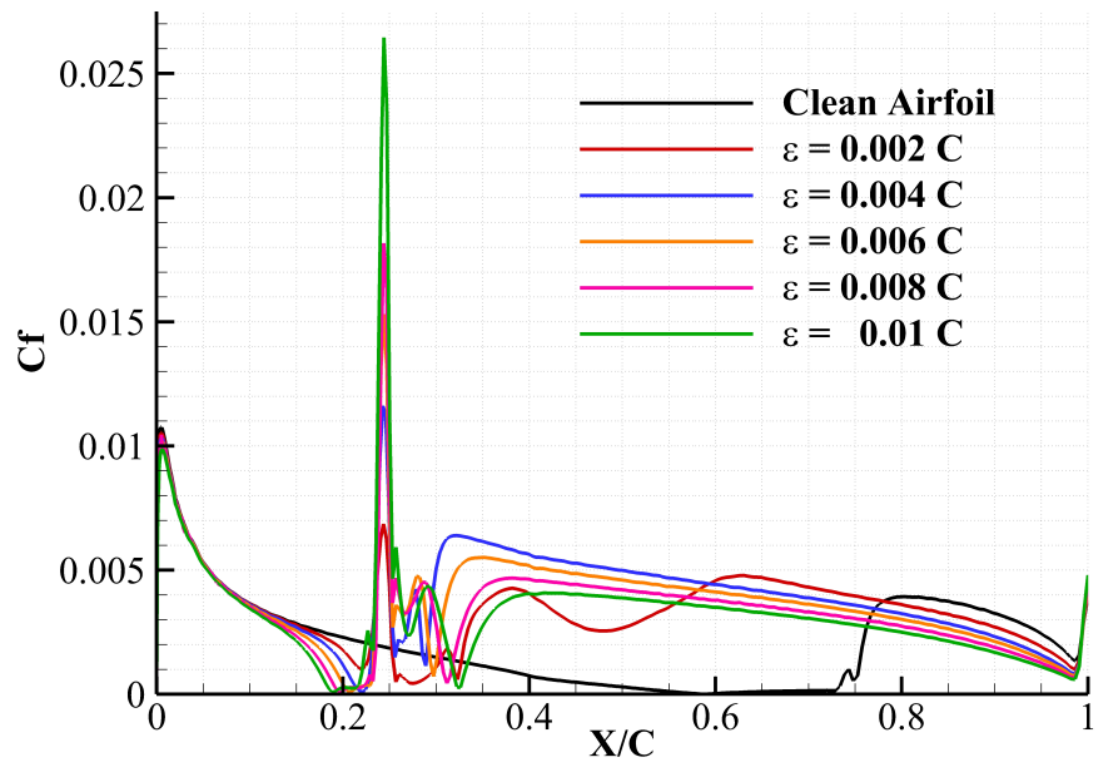

(a)

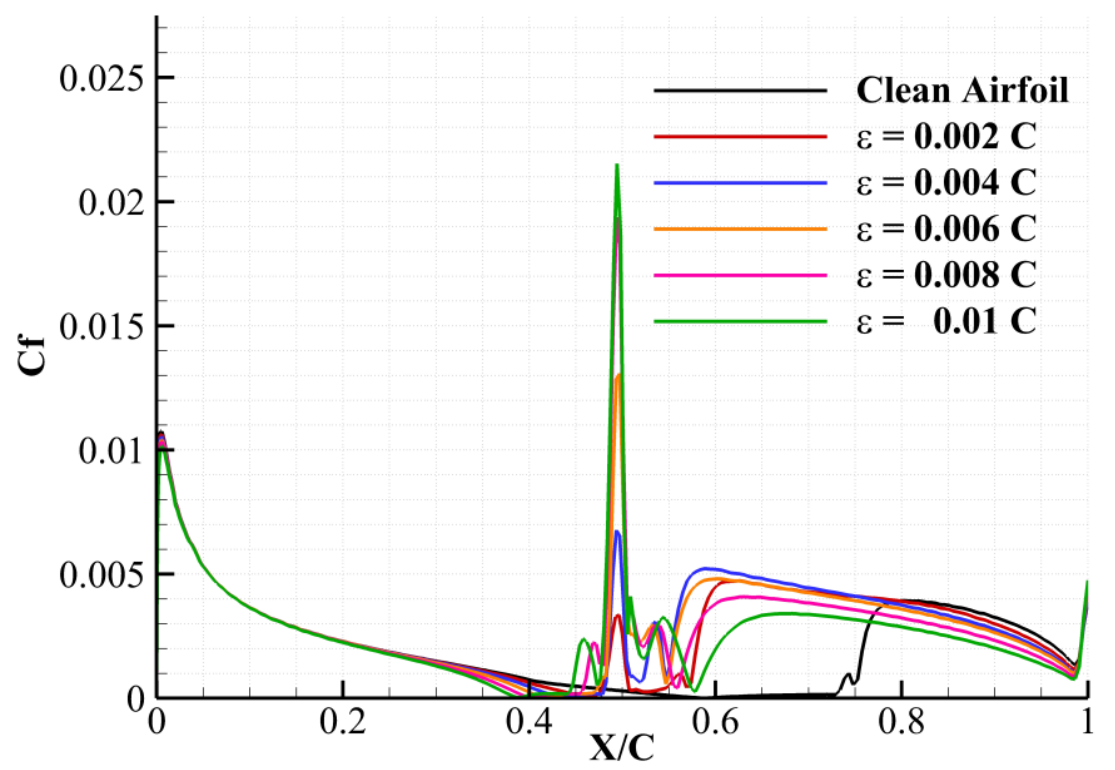

(b) 


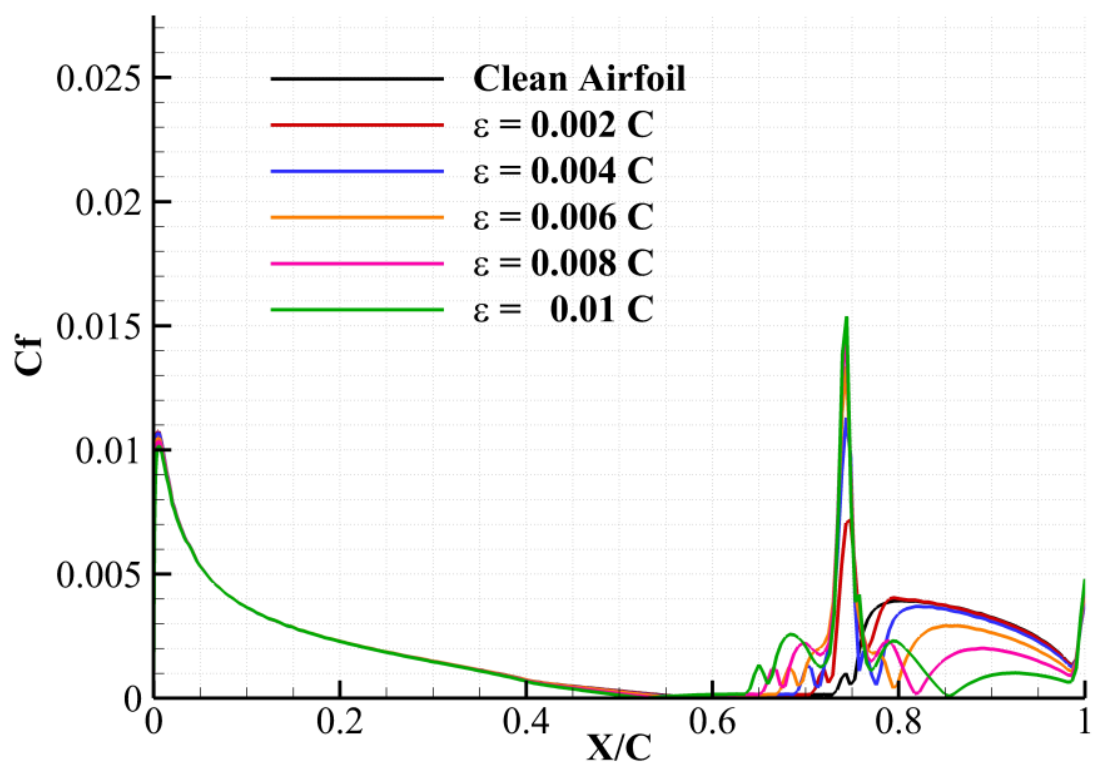

(c)

Figure 5.20. The upper surface skin friction coefficient distribution for different wrinkles heights located at (a) $\mathrm{X}=0.25 \mathrm{C}$, (b) $\mathrm{X}=0.50 \mathrm{C}$ and (c) $\mathrm{X}=0.75 \mathrm{C}$.

For streamlined bodies such as airfoils, the drag coefficient is mainly calculated from the friction drag coefficient which is calculated as the integration of the skin friction coefficient. This means that as the area under the curves in figure (5.20) increases, the drag coefficient value increases. The skin friction coefficient starts from zero at the stagnation point at the leading edge of the airfoil. The skin friction coefficient experiences a sudden increase in value due to the acceleration of the flow over the airfoil's leading-edge curvature. As the flow travels over the airfoil, the boundary layer thickens, the tangential velocity gradients decrease and the wall shear stresses decrease. This continues until the laminar boundary layer experiences a transition in its state from laminar to turbulent. In case of the clean airfoil, this transition occurs approximately at $\mathrm{X} / \mathrm{C} \approx 0.75$. At this location, the velocity gradients increase significantly leading to a sudden increase in the value of the skin friction coefficient. For all the 15 cases with their different wrinkles' heights and locations, the wrinkle has triggered the transition of the boundary layer from laminar to turbulent as shown from the intermittency contour plots in figure (5.21). 


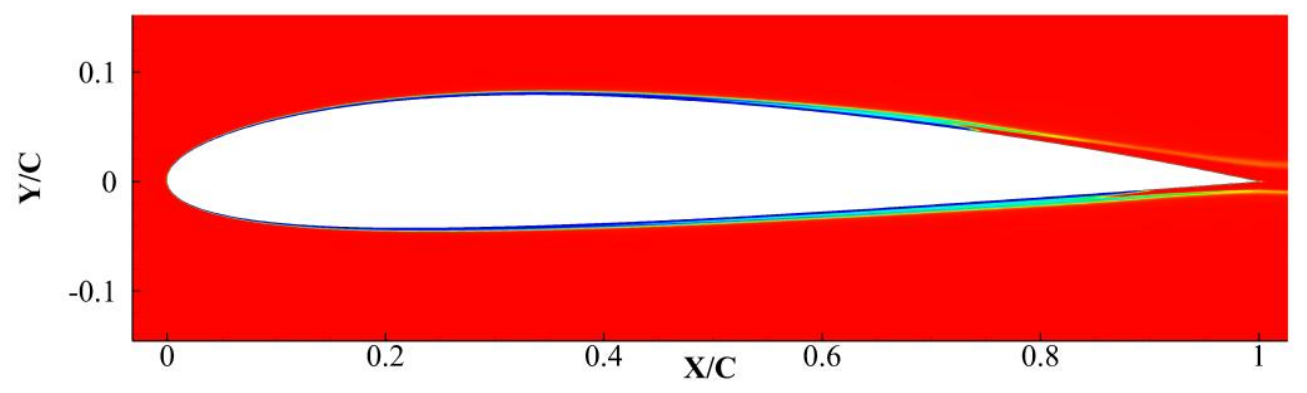

(a)

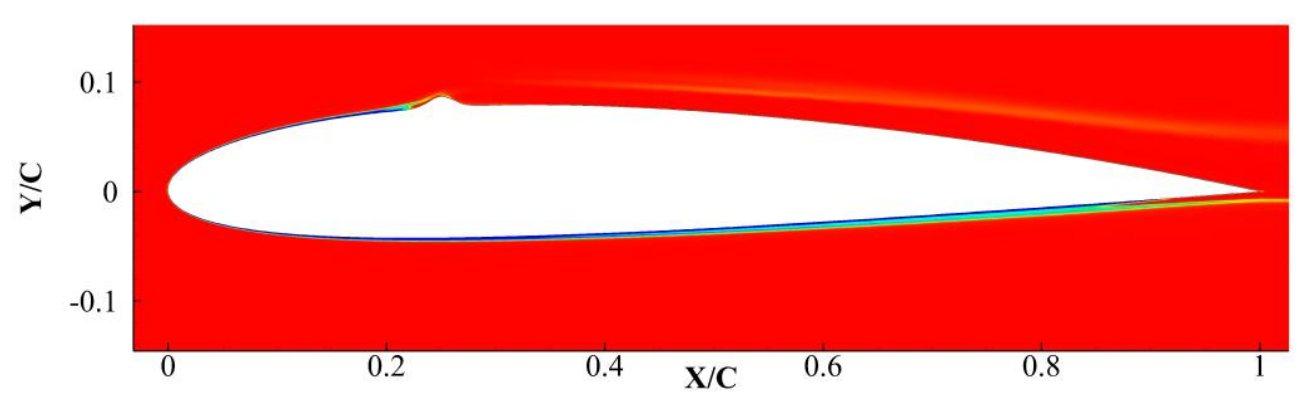

(b)

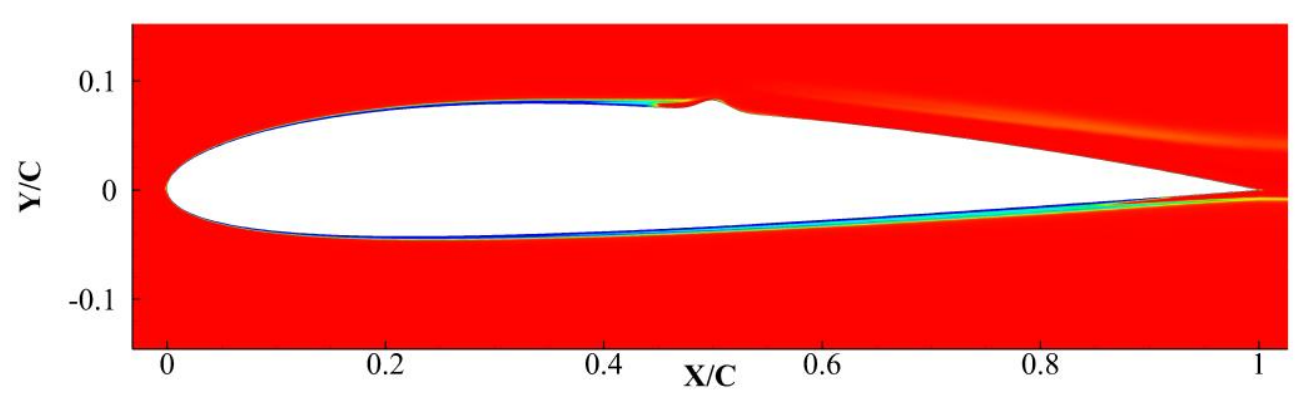

(c)

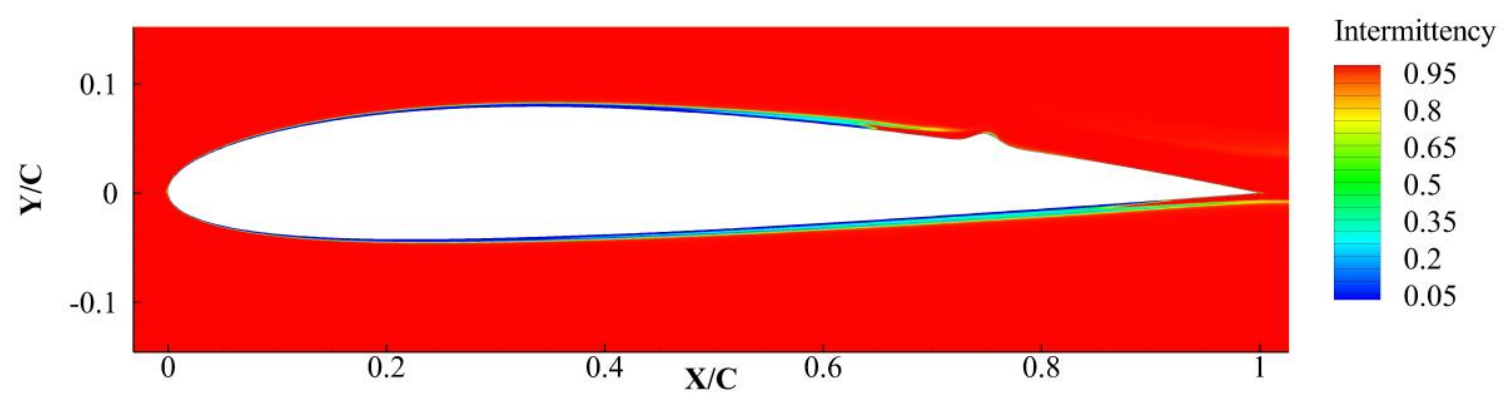

(d)

Figure 5.21. Intermittency contour plot in case of (a) clean airfoil, (b) wrinkled airfoil with the wrinkle at $\mathrm{X} / \mathrm{C}=0.25$, (c) at $\mathrm{X} / \mathrm{C}=0.50$, and at $(\mathrm{d}) \mathrm{X} / \mathrm{C}=0.75$. 
Figure (5.21) shows that regardless of the wrinkle location and height (not shown in the figure), the laminar boundary layer experiences a transition from laminar to turbulent at the location of the wrinkle or at a location slightly upstream of the wrinkle location. This transition of the state of the boundary layer results in increased values of skin friction coefficient as shown in figure (5.20) and consequently increased drag coefficient as shown in figure (5.19).

Combining the degraded lifting capabilities of the wrinkled airfoil with the increased drag force results in a significantly lower lift-to-drag ratio $L / D$ for all the cases of the wrinkled airfoils. Figure (5.22) shows that lift-to-drag ratio calculated for the 15 different configurations of the wrinkled airfoils, with the value of the clean airfoil shown as a dashed line.

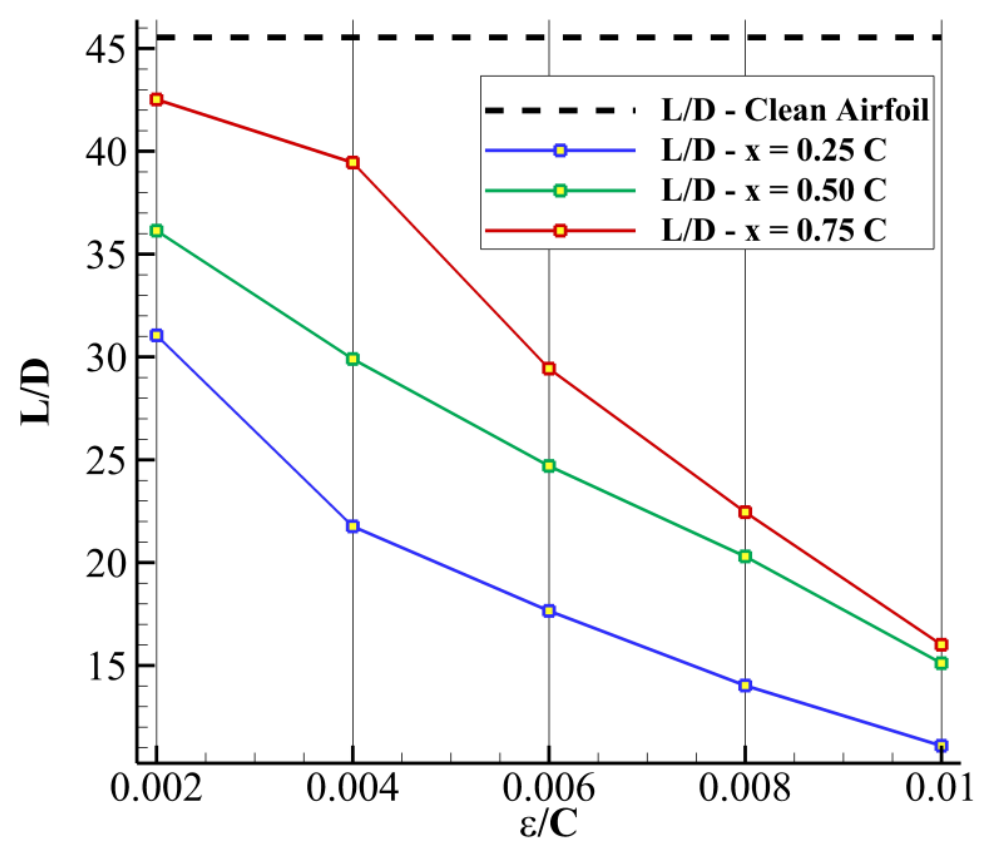

Figure 5.22. The lift-to-drag ratio of wrinkled airfoils with different wrinkle heights and locations along the chord-wise direction of the airfoil.

The lift-to-drag ratio is one of the most important aerodynamic performance metrics. For a particular UAV, the lifting force of the aircraft is dictated by its weight. Having a higher lift-todrag ratio means that the aircraft will experience less drag force for its particular weight requirement, and this is translated to a better fuel economy, an improved climbing performance, and higher glide ratio. Introducing a wrinkle to the upper surface of an airfoil significantly affects the lift-to-drag ratio. For the cases with a wrinkle at $\mathrm{X}=0.25 \mathrm{C}$, introducing a wrinkle with a height of $\varepsilon=0.002 \mathrm{C}$ has decreased to $68.2 \%$ of the lift-to-drag ratio of the clean airfoil at the same flight conditions. For a wrinkle height of $\varepsilon=0.01 \mathrm{C}$, the percentage dropped to $24.4 \%$. As the wrinkle location is shifted towards the trailing edge of the airfoil, the deterioration in the lift-to-drag ratio is slightly mitigated. For the cases with a wrinkle located at $\mathrm{X}=0.50 \mathrm{C}$, the lift-to-drag ratio 
dropped to $79.4 \%$ for a wrinkle height of $\varepsilon=0.002 \mathrm{C}$, and decreased to $33.2 \%$ when the wrinkle height reached $\varepsilon=0.01 \mathrm{C}$. Similarly, for cases with a wrinkle installed at $\mathrm{X}=0.75 \mathrm{C}$, the lift-todrag ratio dropped to $93.4 \%$ and $35.1 \%$ for wrinkle heights of $\varepsilon=0.002 \mathrm{C}$ and $\varepsilon=0.01 \mathrm{C}$, respectively. These results presented in figures (5.17), (5.19) and (5.22) show that the aerodynamic performance of wrinkled airfoils is significantly lower than the performance of a clean airfoil at the same flight conditions, and that introducing a single wrinkle to the upper surface of the airfoil has a prominent degrading effect on its performance. 


\section{CONCLUSION, \\ CONTRIBUTIONS AND FUTURE WORK}

\subsection{Summary of the results:}

This thesis documented the analyses and results obtained while investigating the aerodynamic viability of promising designs of morphing skins. The main research tool used in the analyses was numerical simulations using computational fluid dynamics (CFD).

Chapter 2 of this thesis presented an overview of the numerical algorithms used to resolve the flow over morphing skin. The work of this chapter focused on verifying the accuracy of gradient reconstructions methods when used on different types of meshes. A comparison between the numerical and experimental values of the lift and drag coefficients of a NACA 2412 at different angle of attacks was presented as a general validation test case. Results showed the following:

1) The Green Gauss cell-based (GGCB) method is intrinsically inconsistent and achieves a formal order of accuracy of $0^{\text {th }}$ order when used on arbitrary meshes due to the leading error term that contributes to the exact solution.

2) The only scenario for the GGCB method to achieve a $2^{\text {nd }}$ order accurate solution, is when used on meshes with uniform spacing in the $\mathrm{X}$ and $\mathrm{Y}$ directions.

3) Both the Green Gauss node-based (GGNB) method and the LSCB method can attain a $1^{\text {st }}$ order accurate solution regardless of the mesh geometric properties, and $2^{\text {nd }}$ order accuracy when used on uniformly spaced meshes.

4) In terms of the computational expenses, the GGNB method consumed about 9 - 34\% additional time when compared to the fastest converging method in each test case. Both the GGCB and the LSCB methods consumed nearly the same computational time.

5) Therefore, the LSCB method was used in all CFD simulations in this thesis because of compromising between accuracy and numerical efficiency.

6) The very good agreement that was observed between the numerical and experimental results of the NACA 2412 validation test case reflected the high fidelity and reliability of the CFD results. 
Chapter 3 focused on studying the aerodynamics of another type of sliding skin discontinuity. In this configuration, a trench separates between each panel and the other. It was observed that based on the flow conditions and the length of the trench, some cases experienced a transition in the state of the boundary layer from laminar to turbulent, and in other cases the boundary layer bypassed the cavity as if the flow is traveling over a flat plate. A wide range of cavity dimensions and flow conditions were tested to examine the factors that influence the transition of the boundary layer, and the following conclusions were reached:

7) The non-dimensional number $L / \theta * \sqrt{R e_{\theta}}$ showed a strong correlation with the state of the boundary layer. As long as $L / \theta * \sqrt{R e_{\theta}}$ is less than 600, the laminar boundary layer will bypass the cavity without experiencing any change in its state, but when $L / \theta * \sqrt{R e_{\theta}}$ increases beyond 600 , the laminar boundary layer experiences a transition in its state from laminar to turbulent.

8) This condition $\left(\frac{L}{\theta} * \sqrt{R e_{\theta}}<600\right)$ can be used to design the gaps of the segmented morphing skin.

9) A numerical test case was conducted testing a NACA 2412 airfoil with 5 gaps on the upper surface of the airfoil, and three gaps on the lower surface of the airfoil, all fulfilling the condition $\left(\frac{L}{\theta} * \sqrt{R e_{\theta}}<600\right)$. It was observed that the aerodynamic performance of this trenched airfoil is similar to the performance of the clean airfoil. Only a drop of 3.9\% was observed in the lift-to-drag ratio due to a very small upstream shift in the location of the boundary layer transition.

This shows that the segmented morphing skin whose panels are separated with gaps can be designed using this condition $\left(\frac{L}{\theta} * \sqrt{R e_{\theta}}<600\right)$ to ensure an aerodynamic performance that matches that of a clean airfoil.

Chapter 4 presented the numerical study of the aerodynamics of sliding morphing skins when the panels are overlapping with backward-facing step between each panel and the other. The chapter presented the effect of installing a backward-facing step on either side of an airfoil. For each case the aerodynamic effects of the step location $X / C$, step depth $D / C$ and step angle $\beta$ were investigated, and the following conclusions were reached:

10) A price has to be paid when using sliding morphing skin with a backward-facing step on the suction or pressure side of the airfoil. In comparison to the unchanged airfoil profile, employing a step on either the suction or pressure side of the NACA 2412 airfoil had an adverse effect on its lift coefficient $c_{l}$, drag coefficient $c_{d}$ and critical angle of attack $\alpha_{c r}$.

For a step installed on the upper surface (suction side) of an airfoil: 
11) The lift coefficient $c_{l}$ showed a direct relationship to the location of the step $X_{U} / C$, where the values of the lift coefficient continuously increased by shifting the step location from the leading edge to the trailing edge of the airfoil.

12) The values of drag coefficient $c_{d}$ showed an inverse relationship with the step location $X_{U} / C$.

13) Decomposition of the drag coefficient to its two main components showed a domination of the pressure drag coefficient over the viscous drag coefficient. This means that it is insufficient to use the skin friction coefficient as a sole tool of assessment, and only the pressure distribution curves will explain the relationship between the drag coefficient and the step location.

14) As the step depth increased, the lift coefficient decreased, while the drag coefficient followed a linear-like behavior that increased constantly as the step depth increased.

15) Changing the step angle from $45^{\circ}$ to $-45^{\circ}$ did not have any effect significant effect on the aerodynamic properties.

16) The backward-facing step promoted the onset of stall when installed on the upper surface of an airfoil. The case of a step at $X_{U} / C=0.25$ experienced an earlier stall condition when compared to cases with steps at $X_{U} / C=0.5$ and 0.75 , which also have lower values of critical angle of attack $\alpha_{c r}$ compared to the clean airfoil.

For a step installed on the lower surface (pressure side) of an airfoil:

17) The change in the airfoil thickness caused by the backward-facing step has increased the lift coefficient $c_{l}$ in all configurations by at least $11 \%$ when compared to the clean airfoil at the same conditions.

18) The pressure drag component has noticeably increased the value of the drag coefficients due to the low-pressure recirculation zone that is acting on the vertical wall of the step. This adverse effect is slightly mitigated as the step shifts towards the trailing edge of the airfoil.

19) The backward-facing step has delayed the boundary layer transition from laminar to turbulent flow in cases where the step is located after the natural transition point. This led to a small drop in the viscous drag components for these cases.

20) Increasing the step depth has improved the airfoil's lifting capabilities by at least $6 \%$ for a step depth of $D_{L} / C=0.0075$, and increased with the step depth to reach $17 \%$ for a step depth of $D_{L} / C=0.025$.

21) The near stall behavior of three different stepped airfoil configurations were compared with the clean airfoil. In the stepped configurations, the critical angle of attack $\alpha_{c r}$ was at $16^{\circ}$ which is lower than the value of the clean airfoil by two degrees. 
Therefore, sliding skin with a backward-facing step on either the suction or pressure side will degrade the aerodynamic performance of the airfoil and should be avoided. However, if this backward-facing step cannot be avoided, shifting the step from the leading edge towards the trailing edge, or/and decreasing the step depth can mitigate these adverse effects.

Chapter 5 presented a design for a flexible morphing skin that consists of a highly anisotropic composite material. The matrix material of the composite outer layer was chosen to be silicone, for its proven reliability at a wide range of temperatures. Two fiber materials were tested, the first is the carbon fibers, and the second is aluminum strips. This composite material is supported on major ribs that extend along the chord-wise direction of the wing, and minor ribs that keep those major ribs intact, at the same time, they allow the major ribs to move relative to each other covering the envelope of morphing motion.

22) FEA showed that a cosine shaped underlying mesh can reduce the local stresses and strains, especially for the shear (sweep) motion of the morphing skin.

Silicone rubber composite with embedded carbon fibers and aluminum strips were tested as an outer sealant for the morphing skin. An experimental setup tested the out-of-plane deflection of the design by applying a normal pressure of $16.32 \mathrm{kPa}$ on the morphing skin. Results showed that:

23) Samples with carbon fibers showed lower out-of-plane deflection compared to the samples of the aluminum strips. Out-of-plane deflection could be further improved by increasing the fiber volume fraction in the composite material.

In general, this morphing skin represents a kinematically successful design for a four-degree-offreedom morphing skin. However, a finite element model of the flexible skin was simulated over the morphing motion range, and wrinkles were formed on the flexible morphing skin.

24) A normal Gaussian distribution showed to be a good representation of the shape of the formed wrinkles.

Using a systematic approach, different wrinkles' heights were introduced to the upper surface of a NACA 2412 airfoil at different locations along the chord-wise direction. Results showed that:

25) Introducing a wrinkle to an airfoil adversely and significantly affects the aerodynamic performance of the wing.

26) A wrinkle on the upper surface of an airfoil decelerates the flow, increases the pressure increase on the suction side of the airfoil, and results in lower lifting capabilities of the wrinkled airfoil. This drop reached $34.7 \%$ of the lift coefficient value of the clean airfoil when the wrinkle is introduced at $\mathrm{X}=0.25 \mathrm{C}$ and a height of $\varepsilon=0.01 \mathrm{C}$.

27) This degraded lifting performance is mitigated when the wrinkle is shifted towards the trailing edge of the airfoil and its height decreases. The lift coefficient of an airfoil with a 
wrinkle located at $X=0.75$ and a height of $\varepsilon=0.002 \mathrm{C}$ has increased to $99.5 \%$ of the clean airfoil.

28) Introducing a wrinkle into the laminar boundary layer results in a transition in the state of the boundary layer from laminar to turbulent. This early transition of the boundary layer results in higher shear forces and larger drag coefficient values. In case of a wrinkle located at $\mathrm{X}=0.25 \mathrm{C}$ and a height of $\varepsilon=0.01 \mathrm{C}$, the drag coefficient increased by $267.9 \%$ of the value obtained by the clean airfoil. Shifting the wrinkle to a downstream location of $\mathrm{X}=$ $0.75 \mathrm{C}$ and decreasing its height to $\varepsilon=0.002 \mathrm{C}$ resulted in lower drag forces that are $104.1 \%$ of the clean airfoil.

29) Combining the effect of the dropped lifting capabilities, and the higher drag forces has resulted in a significant degraded lift-to-drag ratios for airfoils with a wrinkled surface. The lift-to-drag ratio dropped by $75.6 \%$ for a wrinkle at $\mathrm{X}=0.25 \mathrm{C}$ and a height of $\varepsilon=0.01 \mathrm{C}$. This percentage improved to $93.4 \%$ by shifting the wrinkle location to $\mathrm{X}=0.75 \mathrm{C}$ and decreasing its height to $\varepsilon=0.002 \mathrm{C}$.

30) In all cases, and regardless of the wrinkle location or height, the aerodynamic preperformance represented by $\mathrm{Cl}, \mathrm{Cd}$ and $\mathrm{L} / \mathrm{D}$ was significantly affected by the introduction of a single wrinkle. Usually during morphing, several wrinkles are formed, resulting in further lower performance.

For that reason, and despite fulfilling the kinematic and structural requirements of a morphing skin, flexible skin is not a viable aerodynamic solution for morphing wings.

As a general conclusion, it is recommended to use the sliding panels design as a solution for the morphing skin problem. Backward-facing steps must be avoided to mitigate the adverse aerodynamic effects listed above. Instead, gaps or trenches can be used between the panels. If the

condition $\frac{L}{\theta} * \sqrt{R e_{\theta}}<600$ is fulfilled, the performance of this morphing skin can match the performance of a clean airfoil 


\subsection{Contributions:}

Most of the findings in this thesis have been published, either as a journal article, or in a conference proceeding. The publications that came out of each chapter are listed below.

\section{Chapter 2:}

In chapter 2, the CFD tool used to carry out the numerical simulations was presented. Unfortunately, there are few articles and documents in literature that have studied the effect of the gradient operator on the CFD solution. For that reason, the majority of chapter 2 has been dedicated to studying the order of accuracy of different gradient reconstruction methods, their compatibility with different types of grids, their efficiency and their effect on the accuracy of the solution. This work has resulted in two conference papers, and one journal article.

1. Mishriky, F., and Walsh, P. (2016) Towards Understanding the Influence of Gradient Reconstruction Methods on Unstructured Flow Simulations. CSME Transactions 41. 2.1 (8 pages).

2. Mishriky, F., \& Walsh, P. (2016) Towards Understanding the Influence of Gradient Reconstruction Methods on Unstructured Flow Simulations. Annual Conference of the CFD Society of Canada-CFDSC '16, Kelowna, British Columbia, June 2016. (8 pages)

3. Mishriky, F., \& Walsh, P. (2016) Observed Order of Accuracy of Gradient Reconstruction Methods. Annual Conference of the CFD Society of Canada-CFDSC '16, Kelowna, British Columbia, June 2016. (6 pages)

\section{Chapter 3:}

This chapter focused on studying the flow over cavities, and validating the numerical results versus experimental data, semi-empirical equations and other benchmark simulations. The high fidelity of the numerical results allowed the author to step up the analyses to derive a scaling law that can predict the critical width of the trench that will trigger the boundary layer transition. The analysis showed that when the trench fulfills the conditions $\left(\frac{L}{\theta} * \sqrt{R e_{\theta}}<600\right)$, the boundary layer bypasses the cavity and maintains its laminar state. This means that sliding panels can be kinematically designed to fulfill this condition resulting in a morphing skin has an aerodynamic performance close to that of a clean airfoil. The work of this chapter resulted in a journal article and conference proceeding. 
4. Mishriky, F. \& Walsh, P. (2017) Prediction of boundary layer transition over cavities and its applications in morphing wings. AIAA journal. (Submitted final revision on June $29^{\text {th }}$, 2018, 28 pages).

5. Mishriky, F. \& Walsh, P. (2017) Aerodynamics of Trenched Airfoils for Morphing Skin Applications. CASI Aero'17 conference, Toronto, Ontario, June 2017. (9 pages) - The best presentation award.

\section{Chapter 4:}

Chapter 4 focused on the aerodynamics of airfoils when a backward-facing step is installed on either side of the airfoil. The aerodynamic effects of the step location, depth, and angle were studied to understand the penalties expected when the panels of the sliding morphing skin shifts in a way that introduces this type of discontinuity. The aerodynamic analysis of this chapter resulted in four journal articles.

6. Mishriky, F., \& Walsh, P. (2016) Effect of the Backward-Facing Step Location on the Aerodynamics of a Morphing Wing. Aerospace, 3, 25 (15 pages - Published).

7. Mishriky, F., \& Walsh, P. (2016) Effect of Step Depth and Angle in Kline-Fogleman (KFm-2) Airfoil. Global Journal of Research in Engineering, 16.1, (7 pages - Published).

8. Mishriky, F., \& Walsh, P. (2016) Effect of Step Depth and Angle on the Aerodynamics of a Sliding Morphing Skin. American Journal of Aerospace Engineering, Vol. 3, No. 3, 2016, pp. 24-30. doi: 10.11648/j.ajae.20160303.11 (8 pages - Published).

9. Mishriky, F., \& Walsh, P. (2016) Aerodynamics of a Backward-facing Step on the Lower Surface of a Sliding Morphing Skin. Advances in Aerospace Science and Technology (18 pages - Under Review).

\section{Chapter 5:}

In order to avoid having discontinuities on the morphing skin, the design and aerodynamics of a flexible morphing skin have been introduced in chapter 5 . The analysis showed that this design is kinematically viable. It can morph in the required degrees of freedom with minimum energy requirements, while maintaining its out-of-plane stiffness. However, during morphing, wrinkles are formed on the surface of the wing resulting in a poor aerodynamic performance. Results of these analysis resulted in two conference proceeding articles, one presents the mechanical design, and the other studies the aerodynamic performance of wrinkled airfoils.

10. Mishriky, F. \& Walsh, P. (2018) Aerodynamics of Flexible Morphing Skin. CFD Society of Canada. '18, Winnipeg, Manitoba, June 2018.

11. Mishriky, F., Walsh, P., \& Xi, F. (2015) Reinforced Cellular Structure Morphing Skin. CASI - AERO '15, Montreal, Quebec, May 2015. (10 pages) 


\subsection{Future Work:}

This section presents some of the future work that can be added to the work present in each chapter. In chapter 2 which focused on the numerical results' validation and verification, a study can be conducted to compare the numerical results of different turbulence models with benchmark experiments. Results can focus on boundary layer transition, flow separation over airfoils, laminar separation bubbles at low Reynolds numbers and shock wave formation at supercritical Mach numbers. These flow features are the main secondary flows that are commonly encountered. Another major limitation in CFD modeling is the turbulence modeling of separated flows. Despite providing several validation test cases for the turbulence model used in chapters 2 and 3, experimental validation of the ability of the transition SST model to capture the physics of shear flows will settle any disputes about this limitation.

The third chapter focused on the geometrical factors of a trench, and their corresponding flow features that will cause the boundary layer to experience transition from laminar to turbulent state over the trench vicinity. One of the main reasons of the growth of the flow instabilities over the trench were the acoustic radiations that are emitted from the trailing edge of the cavity. The author believes that by rounding the trailing edge of the cavity, the acoustic waves can be reflected at different rates than the vortex shedding rate, thus avoiding aero-acoustic resonance. Other factors that was not investigated in this thesis and can affect the stability of the boundary layer are the cavity depth, and the turbulence intensity of the upstream flow.

In chapter 4, additional analysis can be performed on airfoils with backward-facing steps by changing the shape of the step vicinity and restores the original shape of the airfoil after a small distance from the step edge. The author believes that this configuration can mitigate the large pressure drag acting on the step vertical wall. A cascade of backward-facing steps can also be tested to resemble a full morphing airfoil. In addition to this, a modal stability analysis can be performed to relate the step depth and flow variables with the instability modes and their amplification rates. This analysis may reveal ways that can delay the transition of the boundary layer at the step location.

For the fifth chapter, further study of the kinematics of stretchable skin and their patterns of deformation during morphing can be of beneficial use to integrate the aeroelasticity of the design. The morphing motion of flexible skins can produce ripples and wrinkles parallel to the direction of morphing. The author believe that there is a condition that relates the boundary layer Reynolds number momentum thickness to the minimum height for the wrinkle that will allow the laminar boundary layer to maintain its laminar state. A systematic testing of the different winkle heights can yield a non-dimensional number that can predict the transition of the state of the boundary layer. 


\section{APPENDICES}

\section{Appendix A: Steps to run a FLUENT case on Sharcnet server}

To run a FLUENT case on sharcnet, the user can follow these steps:

1. Create the geometry and grid on your local machine.

2. Open ANSYS FLUENT on a local machine (one of the computer labs on campus).

3. Load the grid to FLUENT and set all parameters of the numerical solver.

4. Initialize your case.

5. Export the '.cas' and '.dat' files.

6. Using a word processing software (such as 'Wordpad'), generate a journal file. The journal file is the file that is used on sharcnet to start the simulation and communicate with FLUENT. A simple example of a journal file is shown below:

\begin{tabular}{|l|}
; read files \\
file/read-case "Case1.cas" \\
file/read-data "Case1.dat" \\
; auto-save the data every 50 iterations for example \\
file/auto-save/data-frequency 50 \\
; set the solver courant number \\
solve/set/courant-number 5 \\
S Start the simulation for 10000 iterations \\
solve/iterate 10000 \\
Save the final results \\
file/write-data "data_Unsteady.dat" \\
;exit FLUENT \\
exit yes
\end{tabular}

Figure A. 1. Sample of a script used in a journal file used to start a FLUENT task on sharcnet.

7. Using a secure shell software like 'WinSCP', copy the case and data files to a know folder on the work directory on sharcnet cluster. The cluster must be supporting FLUENT application such as Orca, Redfin and Saw. Please check Sharcnet for a full list of the clusters that can be used for this simulation.

8. Login to a SSH client that supports command line user input such as 'PuTTy'. 
9. Navigate to the location where the case and data files are located.

Ex: cd gwork/User_name/Folder_name

10. To start running the case, the FLUENT module has to be loaded first using the following command:

module load ansys/15.0.7

11. a command line has to be typed specifying the time of this simulation, the number of cores that need to be used, the RAM memory needed for this task, name of the output file, whether the case is $2 \mathrm{D}$ or $3 \mathrm{D}$, specify if double precision is needed, and the name of the journal file that will initiate the simulation.

sqsub -r 3h --nompirun -q mpi -n 16 --mpp=1G -o ofile.\%j fluent 2ddp Journal_file.jou

Command

\begin{tabular}{|l|l|}
\hline -r $\mathbf{3 h}$ & 3 hours of running the simulation task \\
\hline -n $\mathbf{1 6}$ & 16 cores will be used \\
\hline --mpp=1G & 1 G of RAM memory will be used \\
\hline -o ofile.\%j & $\begin{array}{l}\text { The output data will be stored in a file named } \\
\text { 'ofile'. This file will contain all data about the } \\
\text { solution convergence. }\end{array}$ \\
\hline $\mathbf{2 d d p}$ & $\begin{array}{l}\text { The case is 2D and double precision will be used } \\
\text { for the solution. }\end{array}$ \\
\hline Journal_file.jou & $\begin{array}{l}\text { The journal file 'Journal_file' will be used to } \\
\text { start the simulation }\end{array}$ \\
\hline
\end{tabular}

Table A. 1. The command line used to run a FLUENT task on Sharcnet server

12. To check the status of your task, you can type the following command:

sqjobs

The status of your task will be displayed on the command window; Q means that the task is queued, $\mathrm{R}$ means that the task is running, and D means that the task is done. 


\section{Appendix B: General guidelines in generating structured grids for airfoils}

The quality of the mesh is one of the most important element in any CFD numerical simulations. The more time spent in perfecting the grid, the less time the user will have to spend in troubleshooting the results. There are many lessons learnt over the course of this work that may be crucial in obtaining physical and accurate numerical results from the CFD simulator. The purpose of this appendix is to provide the reader with some of general tips when generating a mesh to simulate transonic flows over sharp edge airfoils. The presentation of these general guidelines do not follow any specific guidelines.

\section{- Blocking for a sharp trailing edge airfoil:}

A convenient topology for a sharp edge airfoil is the $\mathrm{C}$ topology where the blocks are wrapped around the airfoil in a $\mathrm{C}$ shape. An example of this grid topology is shown in the figure B.1.

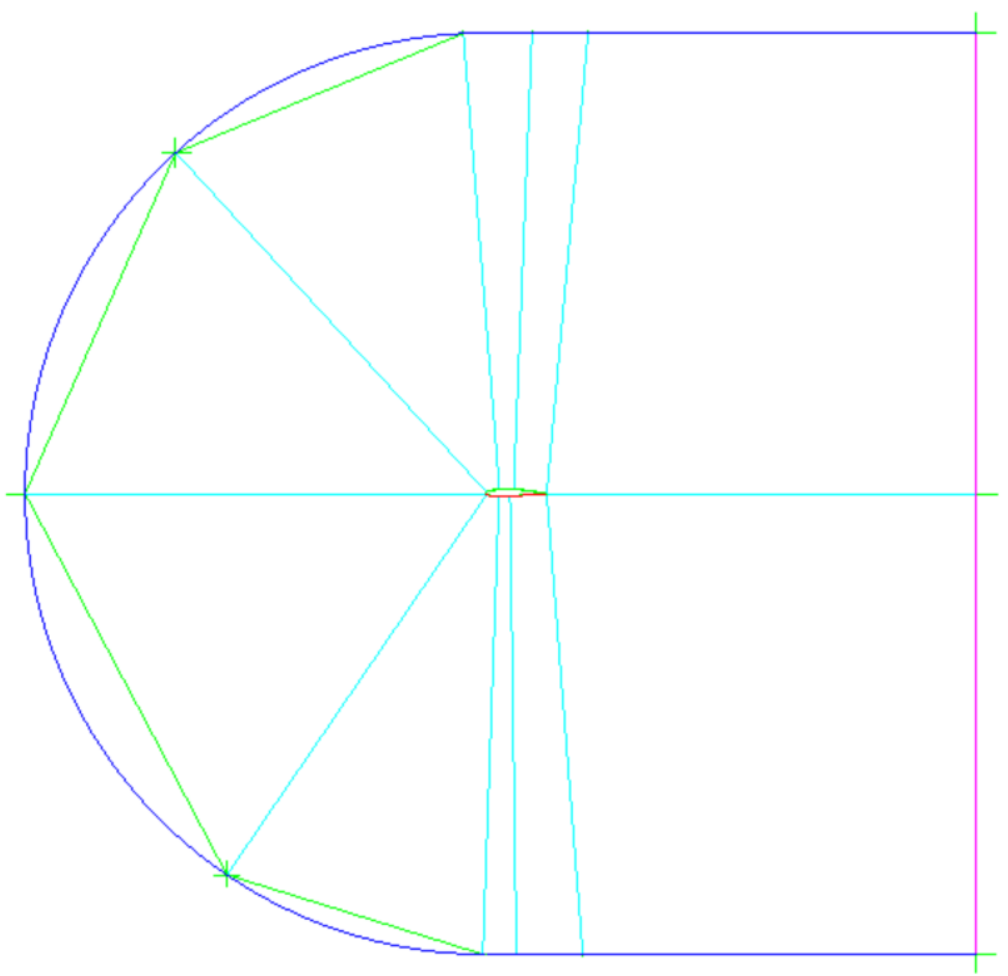

Figure B. 1. C-Grid topology for a NACA 2412 airfoil with a sharp edge trailing edge. 
Sharp edges are usually a location where a there must be split. For sharp edge airfoils, the sharp edge is a place where there must be horizontal split in the grid. Also, it should be noted that every separate zone in the geometry must be covered with blocks that are not shared by any other zone.

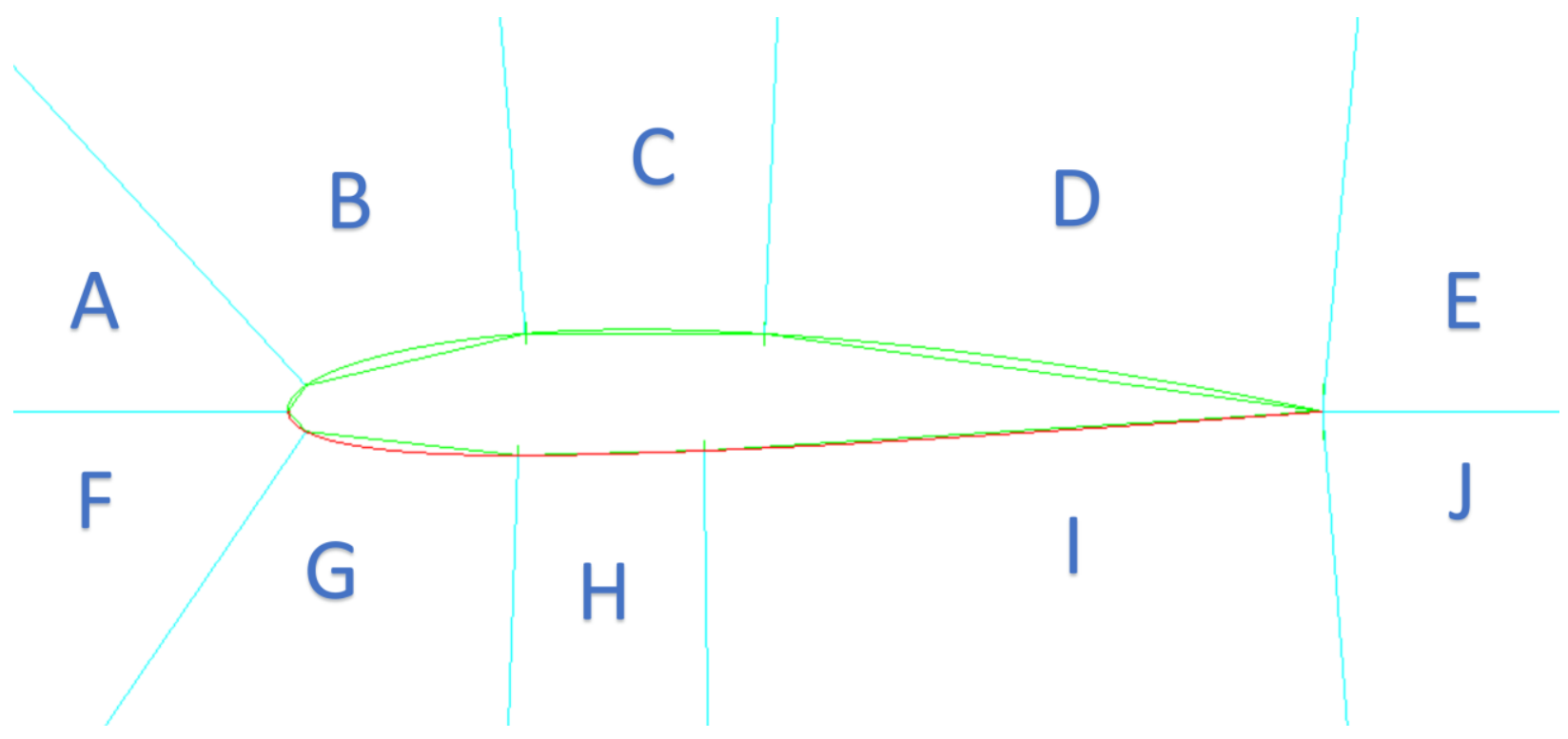

Figure B. 2. Blocks distribution around a NACA 2412.

For example, in figure B.2 the airfoil has two zone, the upper and lower surfaces zones. The upper surface is covered with blocks A, B, C, D, and E, while the lower surface is covered with blocks F, G, H, I, and J. For if block A is shared between the upper and lower surfaces, only one of them will be recognized by FLUENT solver.

\section{- Location of first node and its effect on the $y+$ value}

Another important aspect that has to be carefully considered is the location of the first node close to the wall. Most turbulence models have special treatment for boundary layer and essential condition for this wall treatment to be correctly implemented, the first node of the mesh has to fall within the laminar (or sub-laminar) boundary layer. A general good practice for a proper resolution of the boundary layer is to have a Y+ value less than 1 over the surface of the airfoil. Since the Y+ value is function of the local velocities, the user needs to iteratively place the first node, resolve the flow, check the $\mathrm{Y}+$ value, and repeat until the $\mathrm{Y}+$ value falls below 1 . However, a rule of thumb that the author found useful is using equation (B.1) to calculate the location of the first node.

$$
\Delta y=L Y^{+} \sqrt{74} R e_{L}^{-13 / 14}
$$


Where $\Delta y$ is the location of the first node, $\mathrm{L}$ is the length scale, and $R e_{L}$ is Reynolds number based on this length scale, which for airfoils will be the chord length.

\section{- Expansion ratio should not exceed 1.2}

Placing the first node at a proper location $(\mathrm{Y}+<1)$ does not necessarily guarantee that the boundary layer is resolved well. For that reason, it is important to make sure that the expansion ratio away from the walls is always less than 1.2 , a personal preference is 1.05 for the first few layers of the boundary layer.

- Cluster more nodes at regions of higher curvature. Ex: the leading edge of the airfoil

Now, having the nodes properly placed along the off-wall direction, the spacing between the nodes along the airfoil wall is another important issue. It is important to have enough nodes to properly resolve the curvature of the geometry. This means that at higher curvature more nodes should be used. Figure (B.3) shows how the mesh nodes are clustered at the leading edge of the airfoil.

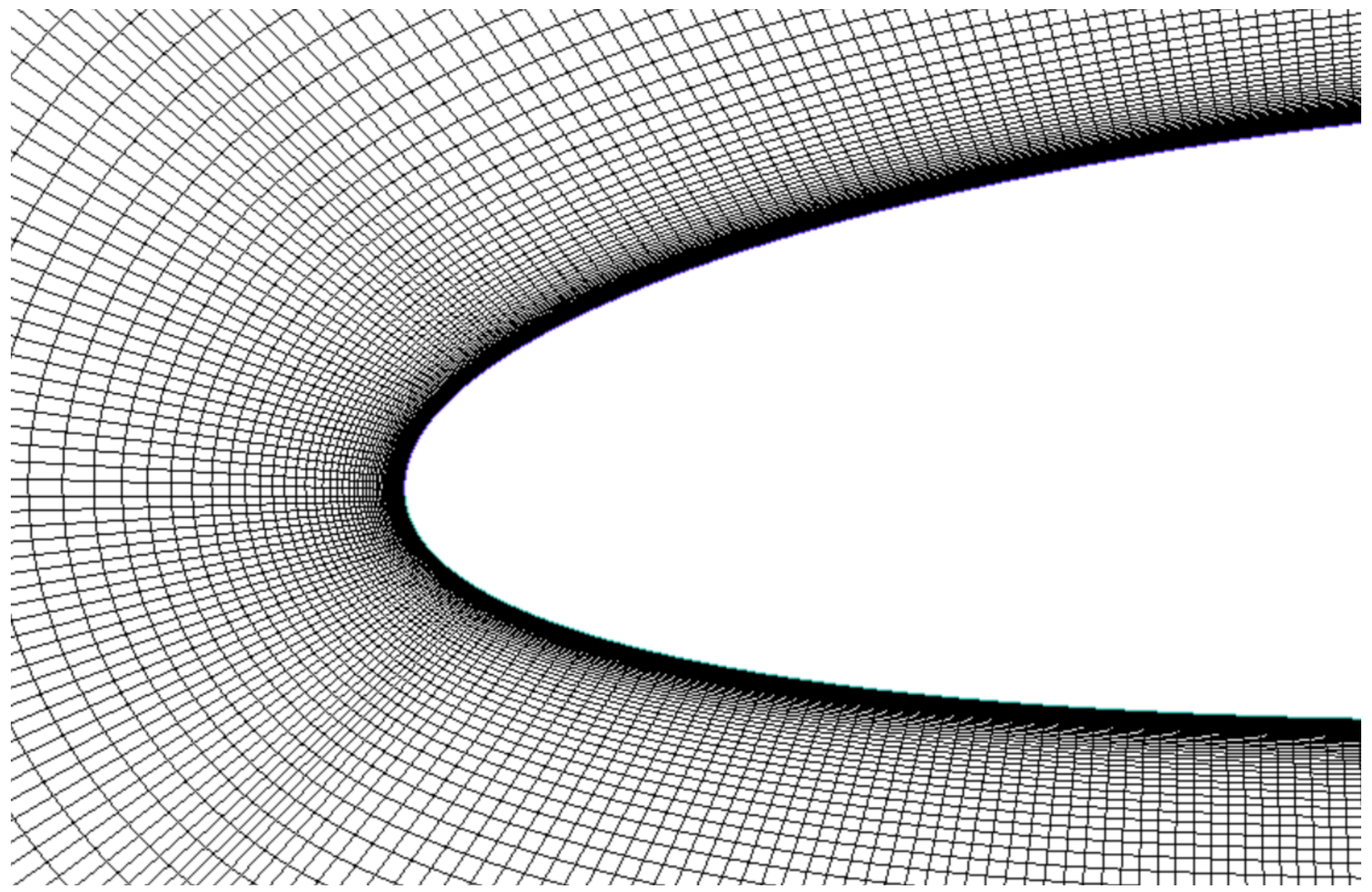

Figure B. 3. Clustering of the nodes at the leading edge of the airfoil to properly capture the geometry's curvature. 
At the end of the day, the mesh of any CFD model has to be treated as a piece of art. Any discontinuity or discrepancy that capture the eyes of the engineer means that there is a potential source of error in the CFD solution. Several tips and books on how to generate the grid will be useful, but it is the engineering judgement that can have the final word. 


\section{REFERENCES}

[1] Metamorphosis. 2017. In Merriam-Webster.com. from https://www.merriamwebster.com/dictionary/metamorphosis [cited: December, 5 2017].

[2] Wickenheiser, A. M., \& Garcia, E. (2007). Aerodynamic modeling of morphing wings using an extended lifting-line analysis. Journal of Aircraft, 44(1), 10-16.

[3] Wickenheiser, A. M, Garcia, E., \& Waszak, M. Evaluation of bio-inspired morphing concepts with regard to aircraft dynamics and performance. In Smart Structures and Materials 2004 Jul 26 (pp. 202-211). International Society for Optics and Photonics.

[4] Joshi, S. P., Tidwell, Z., Crossley, W. A., \& Ramakrishnan, S. (2004). Comparison of morphing wing strategies based upon aircraft performance impacts. sea, 7, 2-32.

[5] Bolonki, A., \& Gilyard, G. B. (1999). Estimated Benefits of Variable-Geometry Wing Camber Control for Transport Aircraft, NASA TM-1999-206586, Oct. 1999.

[6] Lyu, Z., \& Martins, J. (2014). Aerodynamic shape optimization of an adaptive morphing trailing edge wing. In 15th AIAA/ISSMO Multidisciplinary Analysis and Optimization Conference (p. 3275).

[7] Curiale, N. J., \& Zingg, D. W. (2018). Morphing Wings: A Study Using Aerodynamic Shape Optimization. In 2018 AIAA/ASCE/AHS/ASC Structures, Structural Dynamics, and Materials Conference (p. 1910).

[8] Roth, B., \& Crossley, W. (2003). Application of optimization techniques in the conceptual design of morphing aircraft. AIAA Paper, 6733, 2003.

[9] Wlezien, R. W., Horner, G. C., McGowan, A. R., Padula, S. L., Scott, M. A., Silcox, R. J., \& Simpson, J. O. (1998). The aircraft morphing program. AIAA Paper, 1927, 1998.

[10] Barbarino, S., Bilgen, O., Ajaj, R. M., Friswell, M. I., \& Inman, D. J. (2011). A review of morphing aircraft. Journal of intelligent material systems and structures, 22(9), 823-877.

[11] An American Society of Mechanical Engineers. (2006, May). Wing-Folding Mechanism of the Grumman Wildcat

[Online:http://citeseerx.ist.psu.edu/viewdoc/download?doi=10.1.1.693.4216\&rep=rep1\&t ype=pdf]. [cited: December, 5 2017].

[12] Sofla, A. Y. N., Meguid, S. A., Tan, K. T., \& Yeo, W. K. (2010). Shape morphing of aircraft wing: status and challenges. Materials \& Design, 31(3), 1284-1292.

[13] Moosavian, A. (2014). Variable Geometry Wing-Box: Toward a Robotic Morphing Wing (Doctoral dissertation, Doctoral Dissertation, Faculty of Engineering and Architecture Science-Aerospace, Ryerson Univ., Toronto).

[14] Kikuta, M. T. Mechanical properties of candidate materials for morphing wings (Doctoral 
dissertation, Virginia Polytechnic Institute and State University).

[15] Thill, C. L., Etches, J., Bond, I., Potter, K., \& Weaver, P. (2008). Morphing skins. The aeronautical journal, 112(1129), 117-139.

[16] Yu, A., \& Xi, F. J. (2016, August). Design and Analysis of a Sliding Panel Shape Morphing Mechanism System. In ASME 2016 International Design Engineering Technical Conferences and Computers and Information in Engineering Conference(pp. V05BT07A051-V05BT07A051). American Society of Mechanical Engineers.

[17] Yu, A., Xi, F. J., \& Moosavian, A. (2017). A Shape-Morphing Mechanism With Sliding Panels. Journal of Mechanisms and Robotics, 9(4), 041001.

[18] Yu, A., Xi, F., Moosavian, A., \& Li, B. (2018). Design of a Sliding Morphing Skin with Segmented Rigid Panels. Journal of Aircraft, 1-10.

[19] Orr, W. M. F. (1907, January). The stability or instability of the steady motions of a perfect liquid and of a viscous liquid. Part I: A perfect liquid. In Proceedings of the Royal Irish Academy. Section A: Mathematical and Physical Sciences (Vol. 27, pp. 9-68). Royal Irish Academy.

[20] Orr, W. M. F. (1907, January). The stability or instability of the steady motions of a perfect liquid and of a viscous liquid. Part II: A viscous liquid. In Proceedings of the Royal Irish Academy. Section A: Mathematical and Physical Sciences (Vol. 27, pp. 69-138). Royal Irish Academy.

[21] Sommerfeld A. Ein Beitrag zur hydrodynamischen Erklärung der turbulenten Flüssigkeitsbewegung. G. Castelnuovo, ed. InAtti del IV Congresso internazionale dei matematici (pp. 116-124).

[22] Schlichting, H., Gersten, K., Krause, E., \& Oertel, H. (1955). Boundary-layer theory (Ed. 7). New York: McGraw-hill.

[23] Rhie, C. M., \& Chow, W. L. (1983). Numerical study of the turbulent flow past an airfoil with trailing edge separation. AIAA journal, 21(11), 1525-1532.

[24] Yokozeki, T., Takeda, S. I., Ogasawara, T., \& Ishikawa, T. (2006). Mechanical properties of corrugated composites for candidate materials of flexible wing structures. Composites Part A: applied science and manufacturing, 37(10), 1578-1586.

[25] Keihl, M. M., Bortolin, R. S., Sanders, B., Joshi, S., \& Tidwell, Z. (2005, March). Mechanical properties of shape memory polymers for morphing aircraft applications. In Proc. SPIE (Vol. 5762, pp. 143-151).

[26] Kudva, J. N. (2004). Overview of the DARPA smart wing project. Journal of Intelligent Material Systems and Structures, 15(4), 261-267.

[27] Olympio, K. R., \& Gandhi, F. (2010). Flexible skins for morphing aircraft using cellular honeycomb cores. Journal of intelligent material systems and structures, 21(17), 17191735. 
[28] Langtry, R. B., \& Menter, F. R. (2009). Correlation-based transition modeling for unstructured parallelized computational fluid dynamics codes. AIAA journal, 47(12), 2894-2906.

[29] Menter, F. R., Langtry, R., \& Völker, S. (2006). Transition modelling for general purpose CFD codes. Flow, turbulence and combustion, 77(1), 277-303.

[30] Menter, F. R., \& Langtry, R. B. (2012). Transition modelling for turbomachinery flows. In Low Reynolds Number Aerodynamics and Transition. InTech.

[31] Anderson, J. D., \& Wendt, J. (1995). Computational fluid dynamics (Vol. 206). New York: McGraw-Hill.

[32] Baker, T. J. (2005). Mesh generation: Art or science? Progress in Aerospace Sciences, 41(1), 29-63.

[33] Mishriky, F., Walsh P. (2016) Observed Order of Accuracy of Gradient Reconstruction Methods. Annual Conference of the CFD Society of Canada-CFDSC '16, Kelowna, British Columbia, June 2016.

[34] Mishriky, F., and Walsh P. (2016) Towards Understanding the Influence of Gradient Reconstruction Methods on Unstructured Flow Simulations. Annual Conference of the CFD Society of Canada - CFDSC '16, Kelowna, British Columbia, June 2016.

[35] Mishriky, F., \& Walsh, P. (2017). Towards Understanding the Influence of Gradient Reconstruction Methods on Unstructured Flow Simulations. Transactions of the Canadian Society for Mechanical Engineering, 41(2), 169-179.

[36] Holmes, D. G., \& Connell, S. D. (1989). Solution of the 2D Navier-Stokes equations on unstructured adaptive grids (pp. 89-1932). American Institute of Aeronautics and Astronautics.

[37] Rauch, R. D., Batina, J. T., \& Yang, H. T. (1991). Spatial adaption procedures on unstructured meshes for accurate unsteady aerodynamic flow computation.

[38] Sozer, E., Brehm, C., \& Kiris, C. C. (2014, January). Gradient calculation methods on arbitrary polyhedral unstructured meshes for cell-centered cfd solvers. In Proceedings of the 52nd Aerospace Sciences Meeting, National Harbor, MD, USA(Vol. 1317).

[39] Diskin, B., Thomas, J. L., Nielsen, E. J., Nishikawa, H., \& White, J. A. (2010). Comparison of node-centered and cell-centered unstructured finite-volume discretizations: viscous fluxes. AIAA journal, 48(7), 1326.

[40] Diskin, B., \& Thomas, J. L. (2011). Comparison of node-centered and cell-centered unstructured finite-volume discretizations: inviscid fluxes. AIAA journal, 49(4), 836-854.

[41] Mavriplis, D. J. (2003). Revisiting the least-squares procedure for gradient reconstruction on unstructured meshes. AIAA paper, 3986, 2003.

[42] Diskin, B., \& Thomas, J. L. (2012, January). Effects of mesh regularity on accuracy of finite-volume schemes. In 50th AIAA Aerospace Sciences Meeting (pp. 2012-0609). 
[43] Steinbrenner, J. P., \& Abelanet, J. (2007, January). Anisotropic tetrahedral meshing based on surface deformation techniques. In AIAA 45th Aerospace Sciences Meeting.

[44] Abbott, I. H., \& Von Doenhoff, A. E. (1959). Theory of wing sections, including a summary of airfoil data. Courier Corporation, Washington, DC, USA.

[45] Asselin M. (1997) An Introduction to Aircraft Performance; American Institute of Aeronautics and Astronautics, Inc.: Reston, VA, USA.

[46] Zalovcik, J. A. (1944). Profile Drag Coefficients of Conventional and Low-drag Airfoils as Obtained in Flight (No. NACA-WR-L-139). National Aeronautics and Space Admin Langley Research Center Hampton VA.

[47] Fuglsang, D. F., \& Cain, A. B. (1992). Evaluation of shear layer cavity resonance mechanisms by numerical simulation. AIAA Paper, (92-0555).

[48] Bliss, D. B., \& Hayden, R. E. (1976). Landing gear and cavity noise prediction. NASA CR2714, 1976.

[49] Grace, S. (2001). An overview of computational aeroacoustic techniques applied to cavity noise prediction. In 39th Aerospace Sciences Meeting and Exhibit (p. 510).

[50] Roshko, A. (1955). Some measurements of flow in a rectangular cutout (No. NACA-TN3488). California Institute of Technology Pasadena.

[51] Krishnamurty, K. (1955). Acoustic radiation from two-dimensional rectangular cutouts in aerodynamic surfaces. NACA TN-3478.

[52] Rossiter, J. E. (1964). Wind tunnel experiments on the flow over rectangular cavities at subsonic and transonic speeds. Ministry of Aviation; Royal Aircraft Establishment; RAE Farnborough.

[53] Sarohia, V. (1977). Experimental investigation of oscillations in flows over shallow cavities. AIAA J, 15(7), 984-991.

[54] Gharib, M., \& Roshko, A. (1987). The effect of flow oscillations on cavity drag. Journal of Fluid Mechanics, 177, 501-530.

[55] Colonius, T., Basu, A. J., \& Rowley, C. W. (1999). Numerical investigation of the flow past a cavity. positions, 23, 24.

[56] Colonius, T. (2001). An overview of simulation, modeling, and active control of flow/acoustic resonance in open cavities. AIAA paper, 76, 2001.

[57] Rowley, C. W., Colonius, T., \& Basu, A. J. (2002). On self-sustained oscillations in twodimensional compressible flow over rectangular cavities. Journal of Fluid Mechanics, 455, 315-346.

[58] Rizzetta, D. P., \& Visbal, M. R. (2003). Large-eddy simulation of supersonic cavity flowfields including flow control. AIAA journal, 41(8), 1452-1462.

[59] Larchevêque, L., Sagaut, P., \& Labbé, O. (2007). Large-eddy simulation of a subsonic cavity flow including asymmetric three-dimensional effects. Journal of Fluid 
Mechanics, 577, 105-126.

[60] Larchevêque, L., Sagaut, P., Le, T. H., \& Comte, P. (2004). Large-eddy simulation of a compressible flow in a three-dimensional open cavity at high Reynolds number. Journal of Fluid Mechanics, 516, 265-301.

[61] Larchevêque, L., Sagaut, P., Mary, I., Labbé, O., \& Comte, P. (2003). Large-eddy simulation of a compressible flow past a deep cavity. Physics of fluids, 15(1), 193-210.

[62] Chang, K., Constantinescu, G., \& Park, S. O. (2006). Analysis of the flow and mass transfer processes for the incompressible flow past an open cavity with a laminar and a fully turbulent incoming boundary layer. Journal of Fluid Mechanics, 561, 113-145.

[63] Cain, A. B., Rubio, A. D., Bortz, D. M., Banks, H. T., \& Smith, R. C. (2000). Optimizing control of open bay acoustics. AIAA paper, 1928, 2000.

[64] Henderson, J., Badcock, K., and Richards, B. (2000). Subsonic and transonic transitional cavity flows. In 6th Aeroacoustics Conference and Exhibit, pp. 1966.

[65] Shieh, C., \& Morris, P. (2000). Parallel computational aeroacoustic simulation of turbulent subsonic cavity flow. In 6th Aeroacoustics Conference and Exhibit, pp. 1914.

[66] Hankey, W. L., \& Shang, J. S. (1980). Analyses of pressure oscillations in an open cavity. AIAA journal, 18(8) 892-898.

[67] Lamp, A. M., \& Chokani, N. (1997). Computation of cavity flows with suppression using jet blowing. Journal of Aircraft, 34(4), 545-551.

[68] Rona, A., \& Dieudonné, W. (2000). A flow-resonant model of transonic laminar open cavity instability. In 6th Aeroacoustics Conference and Exhibit, pp. 1967.

[69] Shih, S. H., Hamed, A., \& Yeuan, J. J. (1994). Unsteady supersonic cavity flow simulations using coupled k-epsilon and Navier-Stokes equations. AIAA journal, 32, 2015-2021.

[70] Sinha, N., Arunajatesan, S., \& Ukeiley, L. (2000, June). High fidelity simulation of weapons bay aeroacoustics and active flow control. In 6th Aeroacoustics Conference and Exhibit (p. 1968).

[71] Zhang, X., \& Edwards, J. A. (1988). Computational analysis of unsteady supersonic cavity flows driven by thick shear layers. The Aeronautical Journal, 92(919), 365-374.

[72] Zhang, X., Rona, A., \& Edwards, J. A. (1998). The effect of trailing edge geometry on cavity flow oscillation driven by a supersonic shear layer. The Aeronautical Journal, 102(1013), 129-136.

[73] Woolley, J. P., \& Karamcheti, K. (1974). Role of jet stability in edgetone generation. AIAA $J, 12(11), 1457-1458$.

[74] Heller, H. H., \& Bliss, D. B. (1976). Flow-induced pressure fluctuations in cavities and concepts for their suppression. In AIAA Aeroacoustic Conference, NASA Langley Research Center, Hampton, Virginia.

[75] Mack, M., \& McMasters, J. (1992). High Reynolds number testing in support of transport 
airplane development. In 17th Aerospace ground testing conference, Nashville,TN,U.S.A., pp. 3982.

[76] Mishriky, F., \& Walsh, P. (2016). Effect of the Backward-Facing Step Location on the Aerodynamics of a Morphing Wing. Aerospace, 3(3), 25.

[77] Mishriky, F., \& Walsh, P., (2016). Effect of Step Depth and Angle on the Aerodynamics of a Sliding Morphing Skin, American Journal of Aerospace Engineering, 3(3), pp. 2430.

[78] Mishriky, F., \& Walsh, P., (2016) Effect of Step Depth and Angle in Kline-Fogleman (KFm-2) Airfoil," Global Journal of Research in Engineering, Vol. 16, Issue 4, 2016, pp. 1-8. URL: https://engineeringresearch.org/index.php/GJRE/article/view/1491 [cited: December, 5 2017].

[79] Ramrakhyani, D. S., Lesieutre, G. A., Frecker, M., \& Bharti, S. (2005). Aircraft structural morphing using tendon-actuated compliant cellular trusses. Journal of Aircraft, 42(6), 1615-1621.

[80] Fogleman F, Kline R. Airfoil for Aircraft. U.S. Patent No. US 3706430 A, 19 December 1972.

[81] Lumsdaine E, Johnson W. S, Fletcher L. M., \& Peach J. E. Investigation of the KlineFogleman airfoil section for rotor blade applications.

[82] Fertis, D. G. (1994). New airfoil-design concept with improved aerodynamic characteristics. Journal of Aerospace Engineering, 7(3), 328-339.

[83] Finaish, F., \& Witherspoon, S. (1998). Aerodynamic performance of an airfoil with stepinduced vortex for lift augmentation. Journal of Aerospace Engineering, 11(1), 9-16.

[84] Gibson, L. J., \& Ashby, M. F. (1999). Cellular solids: structure and properties. Cambridge university press.

[85] Vocke III, R. D., Kothera, C. S., Woods, B. K., Bubert, E. A., \& Wereley, N. M. (2012). One dimensional morphing structures for advanced aircraft. In Recent Advances in Aircraft Technology. InTech.

[86] Boonvisut, P., \& Çavuşoğlu, M. C. (2013). Estimation of soft tissue mechanical parameters from robotic manipulation data. IEEE/ASME Transactions on Mechatronics, 18(5), 16021611. 